\title{
MECHANISMS OF VEGETATIVE PROPAGATION IN BULBS a molecular approach
}

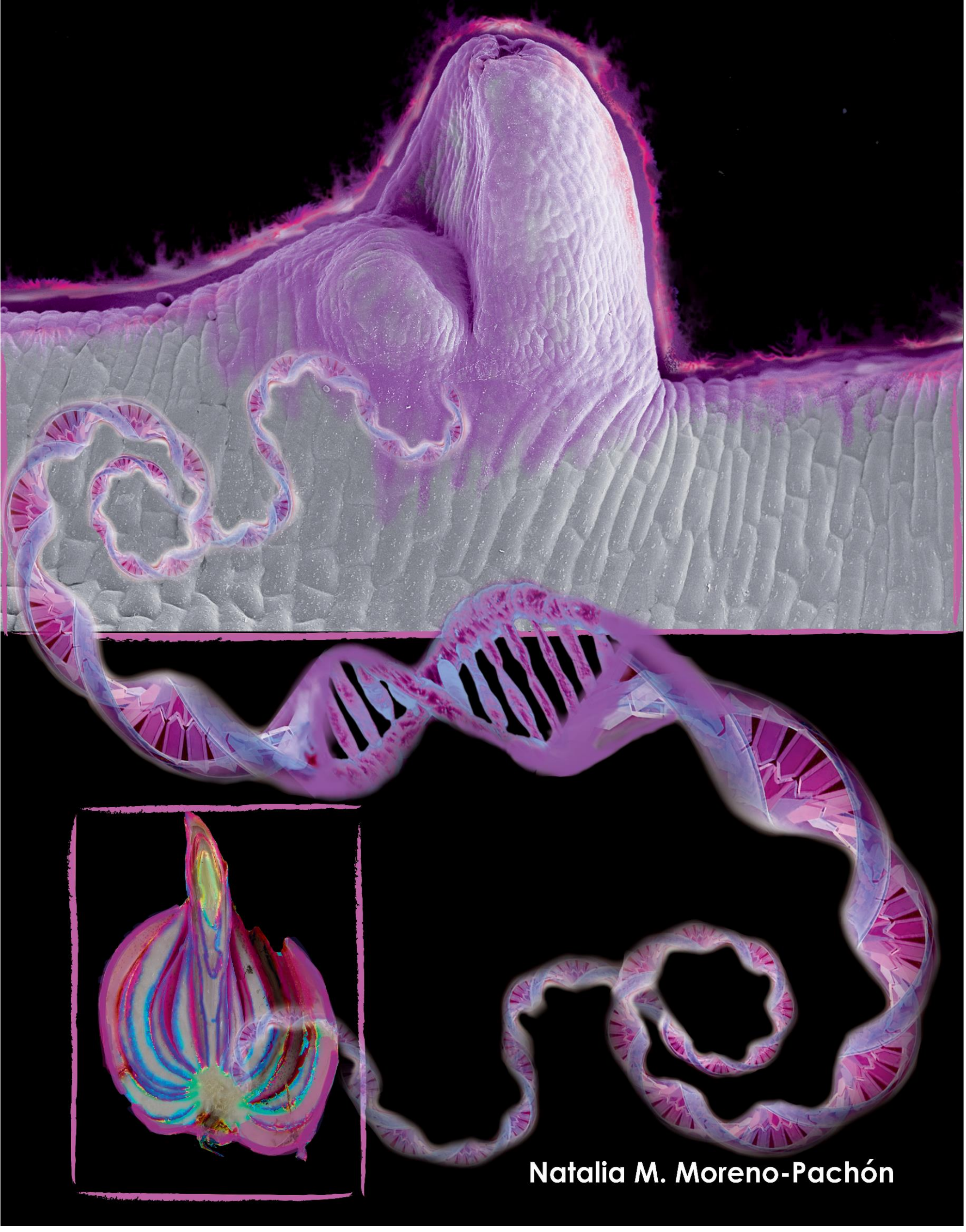




\section{MECHANISMS OF VEGETATIVE PROPAGATION IN BULBS \\ a molecular approach}

Natalia M. Moreno-Pachón 


\section{Thesis committee}

\section{Promotor}

Prof. Dr R.G.H. Immink

Special professor Physiology of Flower Bulbs

Wageningen University \& Research

\section{Co-promotor}

Dr H.W.M. Hilhorst

Associate Professor, Laboratory of Plant Physiology

Wageningen University \& Research

\section{Other members}

Prof. Dr B.J .G. Scheres, Wageningen University \& Research

Prof. DrR. Offringa, University of Leiden

DrC. Philipsen, Dummen Orange, De Lier

DrP.F.P. Arens, Wageningen University \& Resea rch

This research wasconducted under the auspices of the Graduate School Experimental Plant Sciences 


\section{MECHANISMS OF VEGETATIVE PROPAGATION IN BULBS a molecular approach}

Natalia M. Moreno-Pachón

Thesis

submitted in fulfilment of the requirements for the degree of doctor at Wageningen University

by the authority of the Rector Magnific us,

Prof. Dr A.P.J. Mol,

in the presence of the

Thesis Committee appointed by the Academic Board

to be defended in public

on Thursday 12 Oc tober 2017

at 11 a.m. in the Aula. 
Natalia M. Moreno-Pachon

MECHANISMS OF VEG ETATIVE PROPAGATION IN BULBS

a molecularapproach

178 pages

PhD thesis, Wageningen University, Wageningen, the Netherlands (2017) With references, with summary in English

ISBN: 978-94-6343-701-1

DOI: https://doi.org/10.18174/423177 


\section{TABLE OF CONTENTS}

\section{CHAPTER 1}

General introduction

\section{CHAPTER 2}

Transfer of knowledge about flowering and vegetative propagation from model species to bulbous plants

\section{CHAPTER 3}

Elucidating and mining the Tulipa and Lilium transcriptomes

\section{CHAPTER 4}

Role of Tulipa gesneriana TEOSINTE BRANCHED 1 (TgTBI) in the control of axillary bud outgrowth in bulbs

\section{CHAPTER 5}

Molecular analysis of de novo shoot meristem regeneration in lily bulb scales

\section{CHAPTER 6}

Histological origin of regenerated bulblets in lily bulb scales

\section{CHAPTER 7}

General discussion

REFERENCES 

CHAPTER 1

\section{General introduction}




\section{Description of used terms}

Bud: shoot meristem covered by few leaves. A meristem covered by scales in the case of bulbs. For bulbs, bulblet and bulbil are synonyms of bud.

Bulb: main storage organ of bulbous plants.

Bulblet: vegetative adventitious meristem arising from underground organs and covered by a few scales.

Bulbil: vegetative axillary meristem covered by a few scales and located in the axil between a leaf and the stem.

Daughter bulb: New bulb produced in the axil of a scale of the mother bulb through vegetative propagation.

\section{Plant reproduction}

Reproduction, the biological process of creating a new individual, is essential for the survival of the species. While most animals rely on sexual reproduction for this purpose, plants have the capacity to reproduce sexually through seeds or asexually through vegetative shoot meristems (Hartmann 2014). This trait might have been developed in response to sessility, to overcome the mortality by harsh environmental conditions. While seeds can disperse and germinate when the right environmental conditions are met, their mortality is generally higher than plants formed by asexual reproduction, because the mother plant is no longer providing nutrients and mechanical protection (Ikegami et al. 2012). Moreover, plants originating from vegetative meristems are identical to the mother plant, and as such represent clones. Cloning is a desired trait in agriculture and horticulture, allowing to fix and maintain unique desirable genetic compositions.

Vegetative shoot meristems can be categorized according to their developmental process and location on the plant as shoot apical meristems (SAM), axillary meristems and adventitious meristems (Figure 1A). The SAM develops during embryogenesis and is located at the tip of the plant (Long and Barton 2000). Axillary meristems are located in the leaf axils and develop during post-embryonic shoot development into axillary buds that either continue growing into lateral shoots or fall into a period of arrest (Schmitz and Theres 2005), commonly referred to as dormancy. Adventitious meristems arise from differentiated tissues such as leaves, stems or roots (Kerstetter and Hake 1997). Those meristems may originate from a pre-existing group of cells descended from embryonic cells that did not lose their meristematic identity during the development of the plant (Hartmann 2014). This is e.g. the case in Kalanchoe species, commonly known as mother of thousands and producing plantlets from the notches around the leaf margin (Garcês et al. 2007). Adventitious meristems can also originate de novo from 
differentiated tissues that undergo dedifferentiation to create a new population of meristematic cells from where new plant organs will form (Hartmann 2014).

A

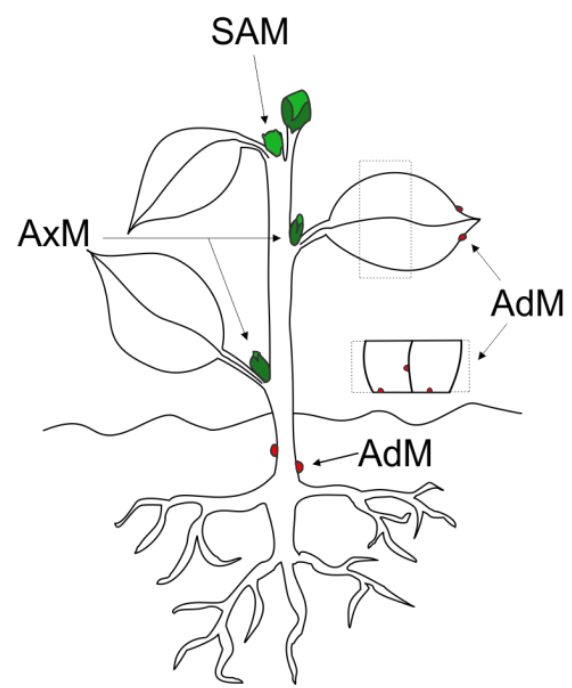

B

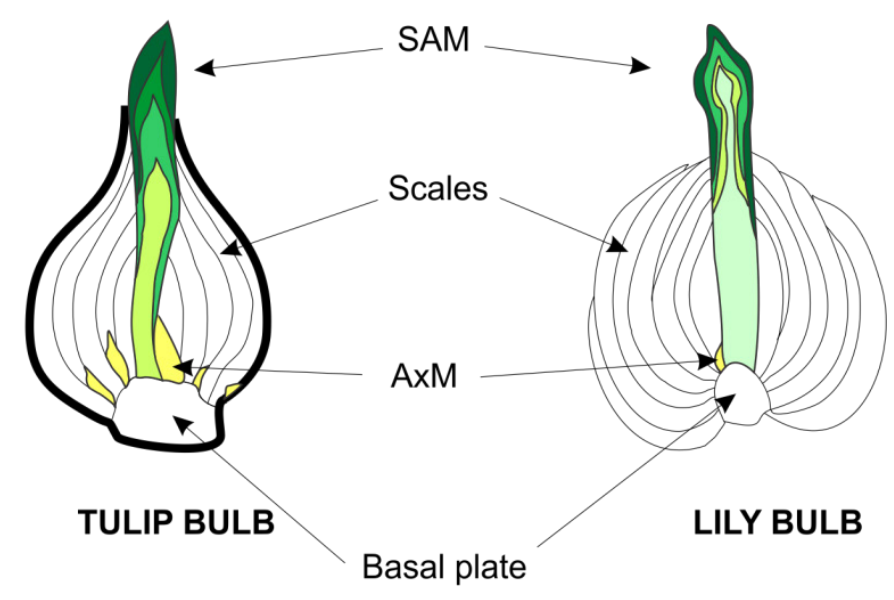

Figure 1. Description of a non-bulbous and a bulbous plant. A. Vegetative meristems in a nonbulbous plant classified based on their developmental process and position in the plant. SAM: shoot apical meristem. AxM: Axillary meristem. AdM: Adventitious meristem. B. Anatomy of tulip and lily bulbs. Bulb are compressed plants where the scales are homolog to the leaves and the basal plate to the stem.

\section{Reproduction in bulbous plant species}

Both axillary and adventitious meristems constitute an important source of vegetative propagation in species where sexual reproduction takes considerably much longer or is not very successful (reviewed in (Leeggangers et al. 2013)). The first case occurs in flower bulbs. Flower bulbs, also known as ornamental geophytes, are herbaceous perennial plants with a bulb as storage organ. Other geophytes have storage organs such as, corms, tubers, rhizomes or enlarged hypocotyls that assure the survival of the plant during winter or dry periods (Hartmann 2014; Kamenetsky 2012; Okubo and Sochacki 2012). Among the diverse geophytes, bulbous plants are of special interest for the Netherlands because of the economic importance of two of its members: lily and tulip (Benschop et al. 2010). In both species the bulb contains specialized scales that serve as the primary storage tissue. Such scales are modified leaves attached to a modified short stem called the basal plate, which at the same time bears roots and at least one axillary bud (De Hertogh et al. 1983; De Hertogh and Le Nard 1993b) (Figure 1B). 


\section{Chapter 1}

\section{What is known about vegetative propagation in tulip and lily?}

Tulip and lily can sexually propagate through seeds but once they sprout, their juvenile and adult vegetative stage can last three to seven years, depending on the genotype and growth conditions, until they finally transition to the reproductive stage (Minas 2007; Shahin et al. 2012). On the contrary, once reached the adult vegetative stage, through vegetative propagation the new plantlets, also referred as bulblets or daughter bulbs, can reach the reproductive state in one growth cycle. On which basis a bulb decides to invest most of its resources in either sexual reproduction via flowers and seeds or vegetative propagation via daughter bulbs or bulblets is not known, but the different reproduction strategies provide these bulbous species a way to adapt to harsh conditions and to optimize survival.

While tulip naturally propagates asexually by the outgrowth of axillary meristems of the mother bulb into daughter bulbs, lily is vegetative propagating mainly by regenerating de novo meristems from the underground stem or roots (Figure 2A-B). One growth cycle of an adult bulb takes a year in both species; however, they differ in the time of flowering. In cultivation, tulip bulbs are planted in late autumn, the shoot apical meristem sprouts by the end of the cold period (winter), the floral bud opens in spring, and the resulting daughter bulbs are lifted from the ground in early summer (De Hertogh et al. 1983) (Figure 2A). On the other hand, lily bulbs are planted in spring, flowering takes place in summer and the daughter bulbs are lifted in early winter (De Hertogh and Le Nard 1993b) (Figure 2B).

A

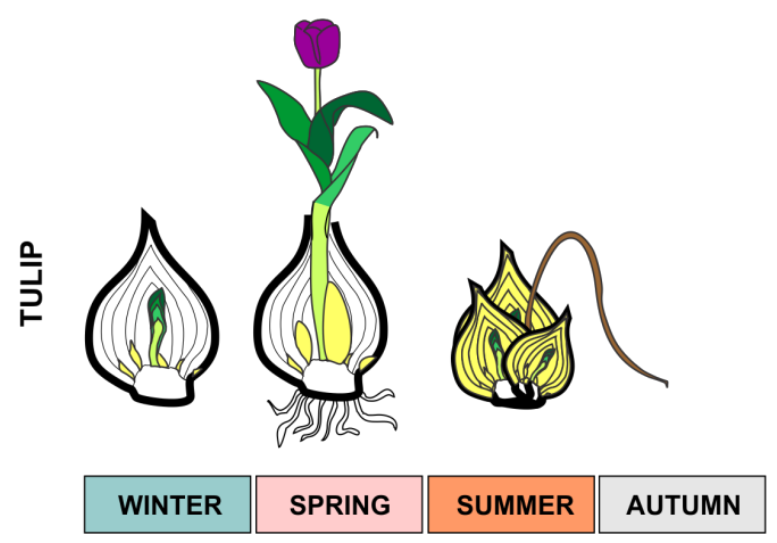

B

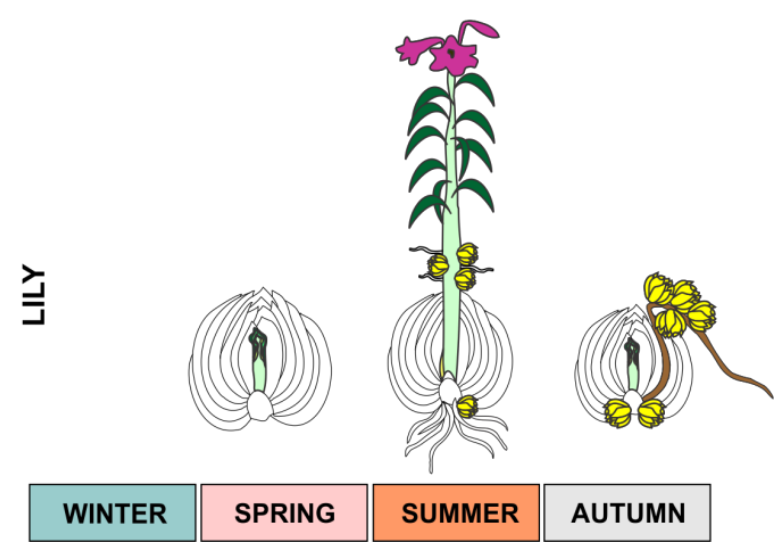

Figure 2. Native vegetative propagation of tulip and lily bulbs. A. Growth cycle of tulip bulbs in relation to their vegetative propagation through axillary bud outgrowth. B. Growth cycle of lily bulbs in relation to their vegetative propagation through adventitious regeneration.

\section{Native vegetative propagation by axillary bud outgrowth in tulip bulbs}

A mature tulip bulb has on average five scales and six axillary buds. The bulb has an apical meristem that according to the age and size of the bulb can be at the vegetative or 
reproductive state. Although all axillary meristems have the capacity to grow into daughter bulbs, on average three daughter bulbs of significant size are formed per bulb every growth cycle (De Hertogh and Le Nard 1993a; Le Nard and de Hertogh 1993; Rees 1966).

One of the best descriptions of the sink to source dynamics in tulip bulbs during the outgrowth of its axillary meristems was done more than 60 years ago by following the distribution of 14- $\mathrm{CO}_{2}$ in the bulb organs (Ho and Rees 1976, 1977). It was found that during the cold period, when the bulbs are thought to be dormant, the scales remobilize part of their stored carbon, from which $75 \%$ is translocated to the roots and SAM. Meanwhile, the rest of the translocated carbon goes to the axillary meristems to support slow growth of the daughter bulbs. When the SAM sprouts and the leaves become physiologically active, they supply carbon to the flower, stem, and daughter bulbs, while the scales exclusively feed the daughter bulbs until they senesce. Once the flower senesces, the carbon source from the still active leaves are translocated into the daughter bulbs and converted into starch (Ho and Rees 1976, 1977). In this process, distribution of carbon resources over the daughter bulbs is not equal and some of the axillary meristems remain dormant for a long period. The exact reason why not all daughter bulbs are released from dormancy after the cold period is not known.

\section{Artificial propagation in tulip bulbs}

Due to the low number of daughter bulbs obtained by axillary bud outgrowth in tulip and the long sexual reproduction cycle, several investigations aiming to improve its regeneration capacity through in vitro culture have been made over the last fifty years (Alderson and Rice 1983; Alderson and Taeb 1990; Baker and Wilkins 1988; Hulscher et al. 1992; Nishiuchi and Koster 1988 ; Podwyszyńska 2005; Podwyszyńska 2006; Podwyszynska and Sochacki 2010; Taeb and Alderson 1990a, b; Minas 2007; van Rossum 1997). All those studies adopted a physiological approach by testing the regeneration response to a plethora of exogenous supplied treatments and cues, including hormones, antioxidants, and different temperature regimes. Although advances have been made in identifying the most competent tissues, e.g. the floral stalk (Rice et al. 1983); and the best inductive treatments, e.g. shoot induction medium followed by low temperature and ending with high sucrose and high temperature (Podwyszynska and Sochacki 2010), the developed protocols are still time consuming and expensive (Figure 3A). 

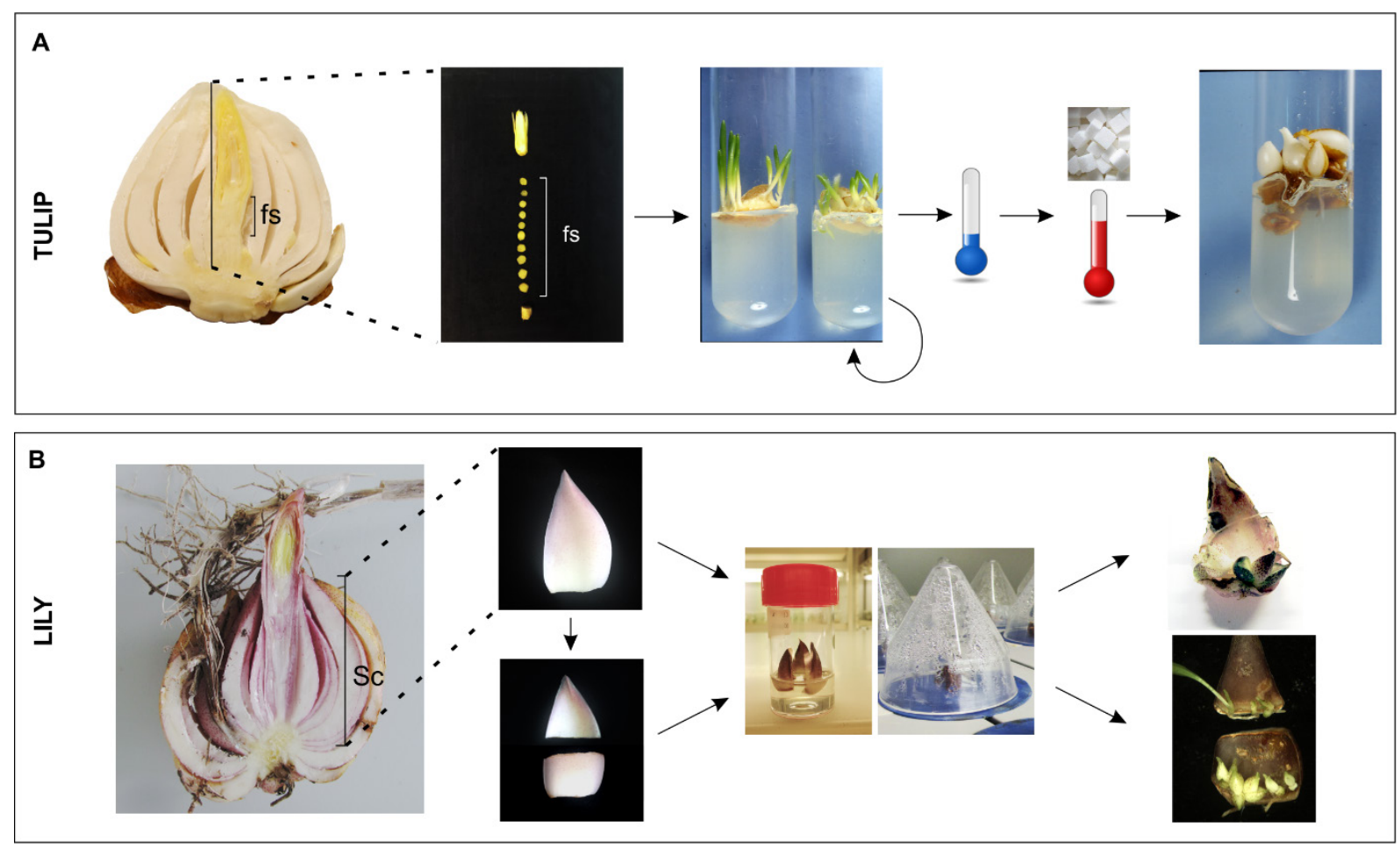

Figure 3. Artificial vegetative propagation in tulip and lily bulbs. A. In vitro regeneration of tulip. The floral stalk (fs) is normally used to regenerate shoots in a shoot inducing medium. Shoots are multiplied, then subjected to cold, and finally treated with warmth and high sucrose. Pictures with blue background were kindly provided by Dr. Geert-Jan de Klerk from the Plant Breeding department of Wageningen University. B. Regeneration in lily using bulb scales (Sc). Regeneration can be achieved in vitro or through scaling. In both cases no exogenous hormones are required. Explants can be cultured entirely or cut into more pieces. In any case, bulblet regeneration occurs at the proximal adaxial side of the explant.

\section{Native and artificial vegetative propagation in lily bulbs}

Contrary to tulip, lily bulbs do not initiate an axillary meristem in the axil of each scale. They rather initiate one axillary bud as a replacement of the senescing apical meristem. Some species produce a bulbil in the axil of the true leaves of the stem and this bulbils fall from the stem and sprout into clones of the mother plant (Bach and Sochacki 2012). But not much attention has been payed to understanding the axillary bud outgrowth in lilies, maybe because this is not the most efficient propagation method of this species. Scaling, on the other hand, is the most commonly used technique to artificially propagate lilies (Bach and Sochacki 2012). Scaling is a propagation method that consists of detaching the bulb scales and culturing them in moist and warm conditions for several weeks. This will result in efficient propagation, because lily bulbs have the capacity to regenerate bulblets at the base of their excised scale (Figure 3B). Many studied have aimed to enhance some physiological traits of lily bulblets by regenerating the bulblets in vitro, including biomass production, repressing dormancy induction and stimulating phase change, (Bonnier and Van Tuyl 1997; Langens-Gerrits et al. 
1997; Langens-Gerrits 2003; Suh and Roh 2014; Gerrits and de Klerk 1992; Jásik and de Klerk 2006; Askari et al. 2016).

A classical series of studies done more than 30 years ago addressed the basic mechanisms granting lily their high de novo regeneration capacity. Through physiological experiments it was shown that exogenous auxins and not cytokinins enhance the number of regenerated bulblets. Moreover, it was shown that a moderate dose of auxin polar transport inhibitors disrupted the position where bulblets normally regenerate (proximal side of the explant) and that high doses inhibited regeneration entirely. By applying different levels of hormones, temperature and wounding, the authors proposed that bulblet regeneration in lily scales is controlled by ethylene production, which might integrate the signals of wounding, temperature and changes in polar auxin transport (Van Aartrijk and Blom-Barnhoorn 1981, 1983, 1984; Van Aartrijk et al. 1986).

\section{What is known about axillary meristem formation and outgrowth in model plant species?}

The success of vegetative propagation through axillary meristems depends on the number of meristems formed and their outgrowth potential. The time of formation of axillary meristems can be determined by the developmental stage of the plant (Grbić and Bleecker 2000), although this can vary according to the species. While in tomato all axillary meristems seem to appear immediately in the leaf axil when the leaf is formed, in Arabidopsis they do not all form at the same time and some leaf axils never form an axillary meristem (Bennett and Leyser 2006). In any case, the axil identity where the future axillary meristem will form needs to be established first. The LATERAL SUPRESSSOR (LAS) transcription factor has this function in Arabidopsis (Greb et al. 2003); LATERAL SUPRESSSOR (LS) in tomato (Schumacher et al. 1999); and MONOCULM 1 (MOC 1 ) in rice (Li et al. 2003). The initiation of an axillary meristem is delimited by the expression of the gene SHOOT MERISTEMLESS (STM). For this reason, the highly conserved STM gene serves as a solid marker for meristem initiation (Long and Barton 2000). From the moment of STM initiation, it is believed that the developmental process of axillary meristem formation follows the program of the SAM.

Once axillary meristems are formed, they normally undergo a period of dormancy until the right conditions trigger their outgrowth (Rohde and Bhalerao 2007). One of these triggers is the loss of the SAM, resulting in a release of apical dominance (Shimizu-Sato and Mori 2001). Several genes have been found to be involved in releasing axillary buds from dormancy, such as RAMOSUS (RMS) in pea, DECREASED APICAL DOMINANCE (DAD) in petunia, MORE AXILLARY GROWTH (MAX) in Arabidopsis; and HIGH TILLERING DWARF (HDT) in rice (Booker et al. 2005; Ishikawa et al. 2005; Liu et al. 2009; Morris et al. 2001; Napoli 1996). Moreover, axillary bud outgrowth seems to be controlled by a complex cross-talk between hormones, nutritional state of the plant and environment (Rameau et al. 2015). Among the hormones known to play a key 


\section{Chapter 1}

role in controlling axillary bud outgrowth (branching), auxin, strigolactone, and abscisic acid act as inhibitors of this process, while cytokinin and gibberellin promote it (Chatfield et al. 2000; Cline 1994; Ferguson and Beveridge 2009; Gomez-Roldan et al. 2008; Napoli et al. 1999; Rameau 2010; Rameau and Pillot 2010; Reddy et al. 2013; Roumeliotis et al. 2012; Shimizu-Sato and Mori 2001; Yao and Finlayson 2015). Changes in the sink to source dynamics in the plant also play an important role in the activation of axillary bud outgrowth. Sucrose has been proposed as an early activator of axillary bud outgrowth when the main sink of the plant, the apex, is removed, making the dormant buds the new sink organs (Mason et al. 2014).

In this complex cross-talk between environment, nutritional state of the plant and hormonal signaling, one mayor integrator has been identified: TEOSINTE BRANCHED I (TB1)/BRANCHED 1 (BRC1) (Rameau et al. 2015). TB1 (monocots)/BRC1 (dicots) is a TCP transcription factor mainly expressed in dormant axillary meristems inhibiting their outgrowth (Poza-Carrión et al. 2007; Takeda et al. 2003; Hubbard et al. 2002). Given the role of TB1/BRC1 as integrator of multiple signals involved in bud outgrowth, mayor attention has been given to this gene in recent years (Martín-Trillo et al. 2011; Nicolas et al. 2015; Braun et al. 2012; González-Grandío et al. 2017).

\section{What is known about de novo meristem regeneration in model species?}

Although plants have totipotent tissues that can be exploited to induce regeneration by the application of auxin and cytokinin, only a few species have been reported to regenerate shoots and roots de novo without exogenous hormone treatments. The term de novo has been used to distinguish between regeneration coming from pre-formed clump of cells with a meristem activity, and regeneration coming from differentiated tissues. In the last case regeneration is thought to occur "from scratch" or de novo (Hartmann 2014). Nevertheless, de novo regeneration can originate from totally differentiated cells or from "less" differentiated pluripotent cells, as e.g. pericycle cells (Atta et al. 2009; Dubrovsky and Rost 2001).

Opposite to vegetative propagation through axillary bud outgrowth, native propagation through de novo shoot meristem regeneration is rare and not a recurrent mechanism in angiosperms (Ikeuchi et al. 2016). Examples of species with the capacity to regenerate entire plants de novo upon excision from the mother plant are Begonia, Sedum (stonecrops), Saintpaulia (african violet), Sansevieria (snake plant), Crassula (Pigmyweeds) and Lilium (Hartmann 2014). Remarkably, those species have not a recent common ancestor and are widely spread in the angiosperm phylogeny (Figure 4), suggesting that native de novo plant regeneration might have evolved independently in those species. 


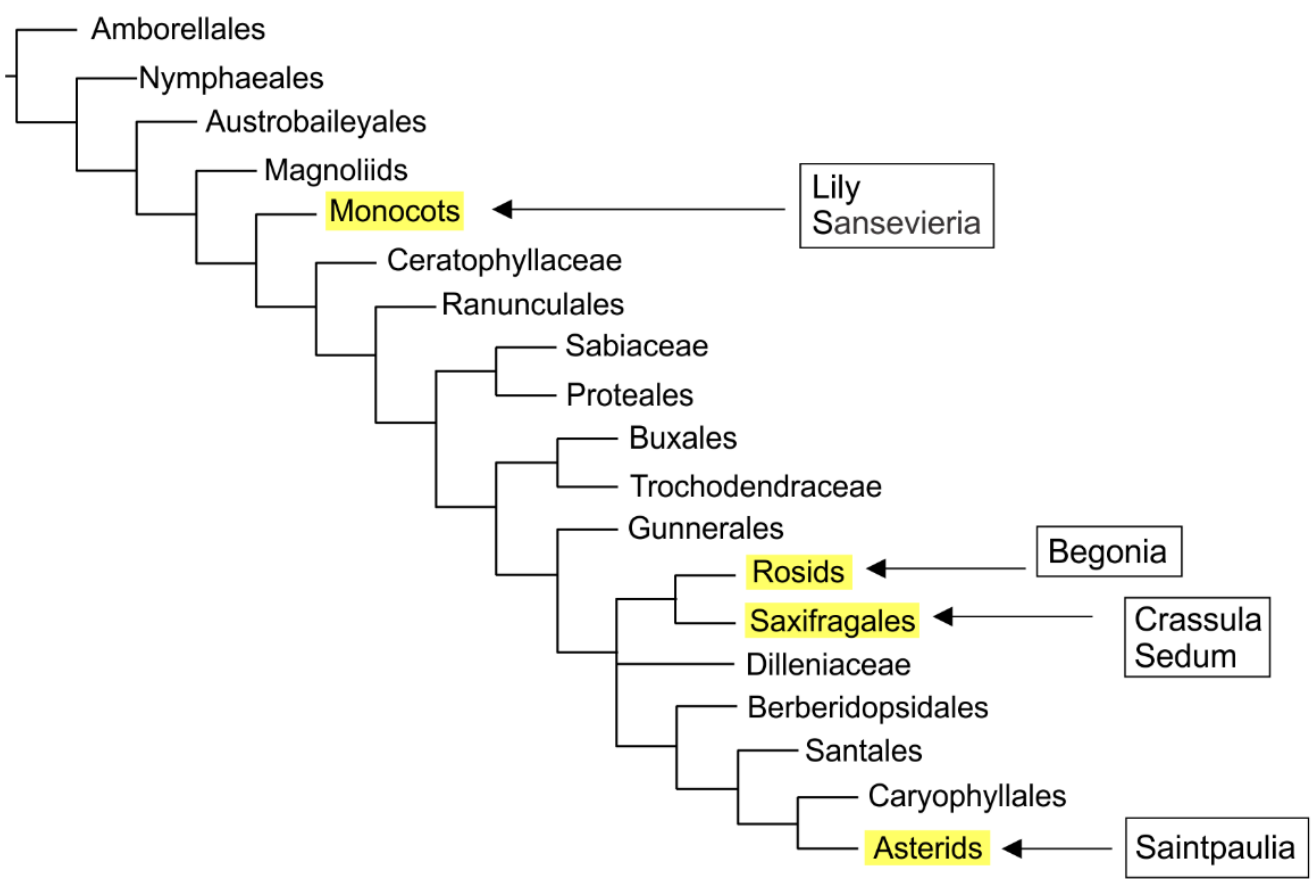

Figure 4. Angiosperm phylogenetic group highlighting the orders of several species that natively can regenerate entire new plants de novo. Modified from (APGIII 2009). The botanical families of each species is as follows: Begonia: Begoniacea; Sedum: Crassulacea; Saintpaulia: Gesneriacea; Crassula: Crassulacea; Sansevieria: Asparagaceae; Lily: Liliacea.

Regeneration in several plant species, including the model plant Arabidopsis, requires an extra induction step, which can be initiated through the manipulation of auxin and cytokinin levels (Duclercq et al. 2011). This step is the formation of callus, which is a tissue formed by excessive and disorganized cell proliferation, and promoted by a high auxin/cytokinin ratio (Motte et al. 2014). During callus formation the competence to regenerate meristems is acquired (Ikeuchi et al. 2013), probably by the expression of PLETHORA 3 (PLT3), (PLT5) and (PLT7), which together promote PLT1-mediated acquisition of pluripotency and CUP-SHAPED 2 (CUC2)-mediated initiation of shoot fate (Kareem et al. 2015). Once transferred to a cytokinin rich medium, WUSCHEL (WUS) expression is induced in high cytokinin responsive domains, in vicinity to low cytokinin responsive regions, expressing CUC2 (Chatfield et al. 2013; Che et al. 2007). As a consequence, a "dome" is arising, which is regarded as a pro-meristem. The polarization of PIN proteins at the periphery of the pro-meristem induces STM expression (Duclercq et al. 2011), which is a hallmark of ectopic meristem establishment.

Interestingly, the formation of callus can also be achieved by transient over expression of WOUND-INDUCED DEDIFFERENTIATION 1 (WINDI) in Arabidopsis (Iwase et al. 2011 a). This gene and its homologs WIND2, WIND3 and WIND4 are activated upon explant excision, thus it can be said that wounding activates WIND genes to promote cell reprograming and callus formation at the wounded sides of the excised explant (Iwase et al. 2011 b). Moreover, entire 


\section{Chapter 1}

plants can regenerate from WIND1-induced callus (Iwase ef al. 2011a). Since the abovementioned species with native de novo regeneration capacity regenerate entire plants upon excision or wounding, it is tempting to speculate that WIND genes grant them their high regeneration capacity. Nevertheless, such species have not been reported to go through a callus state before ectopic meristems arise.

\section{Aim and scope of this thesis}

Tulip and lily, two economically valuable species in the Netherlands, are monocotyledonous bulbous plants, for which vegetative propagation plays a pivotal role in their reproductive success. The limited number of axillary meristems versus the "unlimited" number that can be obtained by de novo regeneration, grant tulip a low propagation rate, while the potential exists for a high rate in lily. Several physiological studies have been performed in the past, aiming to improve the propagation rate in bulbous species. Based on the obtained results it is clear that understanding the fundamental bases of the vegetative propagation mechanism is necessary to set the bases for future applied research. The huge genome (estimated 25,000 Mbp for tulip and 36,000 Mpb for lily) and the lack of molecular tools and resources, make it very challenging to elucidate molecular pathways controlling developmental processes in these two bulbous species. However, it is worthwhile and necessary to pioneer a molecular approach towards the understanding of the molecular control of vegetative propagation in tulip and lily. The aim of this thesis is to understand the mechanisms that control vegetative propagation in each of these bulbous species, more specifically, how axillary bud outgrowth is controlled in tulip bulbs and how regeneration capacity is established in lily bulb scales. A molecular targeted and untargeted approach, respectively, was used to address our main research questions.

In Chapter 2, we reviewed the state of the art of the molecular control of vegetative propagation and flowering time in model species, and how this knowledge may be transferred to bulbous species through bottom-up and top-down approaches. These two biological processes were selected because of their high relevance for agronomical improvements of bulbous plant species.

The aim of Chapter $\mathbf{3}$ was to generate a high quality and as complete as possible transcriptome of tulip and lily in order to build a robust genetic resource for candidate gene approaches. through Illumina RNA sequencing Both transcriptomes were made available in a web-based interface named "Transcriptome Browser" and specific examples of how to mine these huge datasets were given, including an analysis of the TCP transcription factor family, which was the basis for the identification of TgTBI (Chapter 4). Moreover, the risks associated with stringent filtering when assembling de novo transcriptomes is discussed with specific examples. 
In Chapter 4, we aimed at identifying the factors controlling the differential axillary bud outgrowth in tulip bulbs by combining a physiological and targeted molecular approach. The expression of the potential tulip TBI homolog was investigated during the entire tulip growth cycle. Moreover, the role of TgTB1 in controlling axillary bud outgrowth was studied through in vitro assays with administration of exogenous sucrose, cytokinin and strigolactone. It was found that the differential outgrowth capacity in tulip axillary buds is determined by the developmental time of bud initiation in relation to the shoot apical meristem and the dormancy level and sink strength of the buds. Although TgTB 1 is involved in regulating axillary bud outgrowth, it does not seem to be the sole determining factor repressing daughter bulb growth.

In Chapter 5 RNAseq was performed in lily bulb scales in a time series with the aim to characterize the transcriptional changes occurring from explant excision to the appearance of the pro-meristem. Four explants with different regeneration capacity were studied in order to dissect better the developmental and molecular processes underlying de novo shoot meristem regeneration. It was found that wounding activates an extremely fast regeneration response, probably mediated by APETALA2/ETHYLENE RESPONSIVE FACTORS (AP2/ERF). AP2/ERF might in turn mediate polar auxin re-distribution, cell proliferation and cell dedifferentiation. The timing and level of expression of shoot meristem regulators and their correlation with the regeneration capacity of the scale is discussed. This work set the basis for future studies aiming a more thoroughly understanding of de novo shoot regeneration.

The aim of Chapter 6 was to investigate the possible cellular and physiological factors that grant lily bulb scales their competence to regenerate. Based on the performed experiments, we propose that the higher number of secondary veins and higher population of parenchyma cells in the adaxial versus abaxial region of the scale explant confers the adaxial tissues a higher regeneration capacity. Moreover, the important role of the epidermis in directing the position at which the ectopic meristems will arise is highlighted.

Chapter $\mathbf{7}$ consist of a general discussion, in which the generated results in lily and tulip are compared. An integrated analysis was performed to understand how the findings in this thesis contribute to the fundamental and applied understanding of vegetative propagation in bulbous plants. Also, some challenges are discussed; for example, the complexity in the architecture of tulip bulbs and how this impedes ways of improving the rate of axillary bud outgrowth. The challenge to prove the findings of this thesis through functional gene analysis is also discussed, including future possibilities of using transient virus-induced gene silencing (VIGS) for this purpose. Moreover, the potential of lily bulb scales as a model system to study some aspects of de novo regeneration, as well as to study the recalcitrance of in vitro propagation is highlighted, supporting the idea that more "omics" data and biotechnological tools for bulbous plant species are necessary. 



\section{CHAPTER 2}

\section{Transfer of knowledge about flowering and vegetative propagation from model species to bulbous plants}

Hendrika A.C.F. Leeggangers\#, Natalia M. Moreno-Pachon\#, Henk Gudde, Richard G.H. Immink

\# Both authors contributed equally to this work

Published in The International Journal of Developmental Biology, 2013;57(6-8):61 1-20. DOI: 10.1387/ijdb. 130238ri. 


\begin{abstract}
The extensive characterization of plant genes and genome sequences summed to the continuous development of biotechnology tools, has played a major role in understanding biological processes in plant model species. The challenge for the near future is to generate methods and pipelines for an efficient transfer of this knowledge to economically important crops and other plant species. In the case of flower bulbs, which are economically very important for the ornamental industry, flowering time control and vegetative propagation constitute the most relevant processes for agronomical improvements. Those processes have been reasonably studied in reference species, making them excellent candidates for translational investigations in bulbous plant species. The approaches that can be taken for the transfer of biological knowledge from model to non-model species can be roughly categorized as "bottom-up" or "top-down". The former approach usually goes from individual genes to systems, also known as a "gene-by-gene" approach. It assumes conservation of molecular pathways and therefore makes use of sequence homology searches to identify candidate genes. "Top-down" methodologies go from systems to genes, and are e.g. based on large scale transcriptome profiling via heterologous microarrays or RNA sequencing, followed by the identification of associations between phenotypes, genes, and gene expression patterns and levels. In this review, examples of the various knowledge-transfer approaches are provided and pros and cons are discussed. Due to the latest developments in transgenic research and next generation sequencing and the emerging of systems biology as a matured research field, transfer of knowledge concerning flowering time and vegetative propagation capacity in bulbous species come into sight.
\end{abstract}

Keywords: bulbous plant, flowering time control, vegetative propagation, gene regulation 


\section{Introduction}

In the last decade the establishment of full genome-sequences and the development of new biotechnology tools have dramatically increased our knowledge of plant functioning. For example, the genome sequence of Arabidopsis ( $130 \mathrm{Mbp}$; dicot), rice ( 380 Mbp; monocot) and maize ( $2500 \mathrm{Mbp}$; monocot) were completed in 2000, 2002 and 2009, respectively (AGI 2000; Sequencing Project International Rice 2005; Schnable et al. 2009). Molecular biology, genomic and transgenic research, such as loss-of-function mutagenesis and overexpression studies, have played a key role in exploiting and understanding biological and molecular functions of the thousands of genes present in the genome sequences. Nonetheless, the majority of these functional studies have been performed in plant model species, such as Arabidopsis, Medicago and rice. All together this provided a wealth of knowledge on the control of a large variety of biological processes and traits. Hence, the road has been paved for the implementation of this data and the transfer of knowledge from model species to relevant but less studied crop species, ultimately aiming to improve and optimize yield and quality for a sustainable agriculture.

Almost all bulbous plant species are monocots, including the economically important ornamentals tulip and lily. Bulbous plants are hardly studied at the molecular and biotechnological level and therefore this review will have a special focus on these species. Bulbous species were introduced in Western Europe in the $16^{\text {th }}$ century and are nowadays primarily utilized for commercial bulb production, garden and forced fresh cut flower production and for landscape architecture. Cultivation occurs in temperate climate regions with the Netherlands being the leading producer world-wide. In total, seven species dominate the industry consisting of Tulipa, Lilium, Narcissus, Gladiolus, Hyacinthus, Crocus and Iris (Benschop et al. 2010). Flower bulbs propagate sexually through seeds and vegetative via initiation and outgrowth of axillary meristems, which are usually located in the underground storage organ (Kamenetsky and Okubo, 2012). Like other plants, bulbs propagated from seeds undergo three developmental phases: juvenile vegetative, adult vegetative and reproductive. The duration of the juvenile vegetative phase can take several years (e.g. Tulipa and Narcissus) and only upon the transition to the adult vegetative phase, the bulb becomes competent for flower initiating signals. The vegetative phase switch from juvenile to adult depends on the physiological age, weight and size of the bulb. Subsequently, taking tulip as an example, high temperatures can induce the transition from adult vegetative to the reproductive phase, resulting in flower bud initiation. Simultaneously, dormancy is triggered and a pro-longed period of cold is required for dormancy release and internal preparation for stem elongation and flower outgrowth in the next spring. This specific life cycle is not only seen in tulip, but is common for various bulbous species, including Tulipa, Crocus and Hyacinthus (Rees 1966; Saniewski et al. 2000; Kamenetsky et al. 2012). 
In order to improve bulb productivity and ornamental characteristics, it is necessary to increase genetic variation by breeding new cultivars and potentially this can highly benefit from the implementation of biotechnological and 'omics' tools. Currently, the development of a new tulip cultivar can take up to 20 years because of its long juvenile phase and low vegetative propagation rate (Podwyszyńska 2005). Besides the long juvenile phase, which slows down the breeding process and the production of flowers, an agricultural problem is laid down in the precocious flower initiation by high temperatures in spring, resulting in early development of the flower bud. Consequently the flower bud is completely developed inside the bulb around harvest time, leading to either flower abortion or a decrease of flower quality in the next season because of dehydration during storage of the bulbs (Hartsema 1961). In addition, natural vegetative propagation rates vary among flower bulbs, but on average are low due to the limited number of axillary meristems and a restriction in outgrowth of these meristems (Kamenetsky and Okubo, 2012). Together with the long juvenile phase, this makes the development of a new flower bulb cultivar a slow and time consuming process. Many efforts in understanding and improving the physiological nature of flowering and vegetative propagation in bulbous plants took place in the last decades (Beijer 1952; Aung and Hertogh 1979; Lambrechts et al. 1994; Balk and de Boer 1999; Rietveld et al. 2000); however, the majority of these studies focused on physiological factors and limited molecular and genomic studies have been performed. Although various reasons can be brought forward for this, the large genome sizes for bulbous plants (Tulip $\sim 25000 \mathrm{Mbp}$; Lily 36000 Mbp) and technical difficulties in isolating e.g. RNA from bulb scales have been particularly decisive in this (Shahin et al. 2012a).

Here, we will briefly summarize the current knowledge on flowering time control and vegetative propagation gained from studies in model plant species, since these are the two most important biological processes for agronomical improvements of bulbous plant species cultivation. Subsequently, we will give an overview of approaches to transfer this type of knowledge from model plants to crop species and how transgenic and 'omics' technologies can be supportive. Various examples will be given from studies that used such a strategy, including an overview of the technologies that are relevant for bulbous plant species. In the final concluding section a prospect will be given how novel emerging technologies, bioinformatics, and systems biology can increase the efficiency and strength of this type of research and move the field from gene-by-gene approaches into a comprehensive genomewide level. 


\section{What is known on flowering and vegetative propagation from model systems?}

Although the best studied model system, Arabidopsis, is a dicot, and the majority of bulbous plant species are monocots, the regulatory mechanisms underlying important agricultural traits appeared to be conserved in various cases. Hence, knowledge gained in Arabidopsis can be informative for studies in bulbous plants. Strong conservation between Arabidopsis and the monocot rice was observed e.g. for the genes involved in the photoperiod flowering time pathway (Izawa et al. 2003). The same holds for various hormonal signalling components and the key transcription factors involved in axillary meristem formation and outgrowth, which is directly related to vegetative propagation capacity in bulbous species (Finlayson 2007; Kebrom et al. 2013). Nevertheless, various exceptions are known and in general best results are obtained when using a closely related model species as starting point. Therefore, we will discuss mainly knowledge gained from Arabidopsis, but when relevant, complemented with information from other dicots and monocot species.

\section{Vegetative propagation}

Shoot branching is a vegetative process determined by axillary meristems and it determines the architecture, biomass and reproductive success of a plant. Initiation of an axillary meristem results in the formation of a bud that will undergo a period of dormancy. Once the right environmental or endogenous plant factors release the bud from dormancy, it will grow and develop into a branch or a propagule in the case of flower bulbs (e.g. daughter bulb, bulblet, bulbil), a process known as bud outgrowth. Hence, the processes of axillary bud initiation and axillary bud outgrowth together determine the vegetative propagation rate in bulbous species (Fig. 1).

Several genes promoting axillary bud initiation have been identified in different model species (Bennett and Leyser 2006; Kebrom et al. 2013) and their supposed functions could be confirmed by transgenic approaches. For instance, a transcription factor of the GRAS family characterized in tomato, rice and Arabidopsis, - Lateral suppressor (LS), Monoculm l (MOC1) and LATERAL SUPPRESSOR (LAS), respectively - is responsible for the establishment of an axil identity and maintenance of meristematic capacity via prevention of cell de-differentiation (Bennett and Leyser 2006; Greb et al. 2003; Li et al. 2003a; Schmitz and Theres 2005; Ward and Leyser 2004). A second key regulatory gene discovered in tomato, BLIND (BI), encodes a MYB transcription factor that also promotes axillary bud initiation but its function is independent of Ls. Occurrence of bud outgrowth depends on the factors that release buds from dormancy. Apical dominance, which is the ability of the shoot apex of the plant to prevent outgrowth of axillary meristems, and therefore branching, is one of the most studied phenomena controlling dormancy in axillary buds. 


\section{Chapter 2}

The $\mathrm{Bl}$ ortholog in Arabidopsis is REGULATOR OF AXILLARY MERISTEMSI (RAXI) (Keller et al. 2006; Müller et al. 2006). A third regulator identified in Arabidopsis, REGULATOR OF AXILLARY MERISTEM FORMATION (ROX) has orthologs in rice LAX PANICLEl (LAXI) and maize Barren stalk1 (Bal), although the latter two also affect inflorescence branching (Yang et al. 2012). During vegetative development in Arabidopsis, LAS and RAXI influence the expression of ROX and axillary bud initiation occurs when ROX expression ceases (Yang et al. 2012). In contrast, LAX 1 transcripts in rice are detected only after the axillary bud has initiated (Oikawa and Kyozuka 2009), suggesting that the molecular control of ROX-like genes may differ in timing between monocots and dicots.

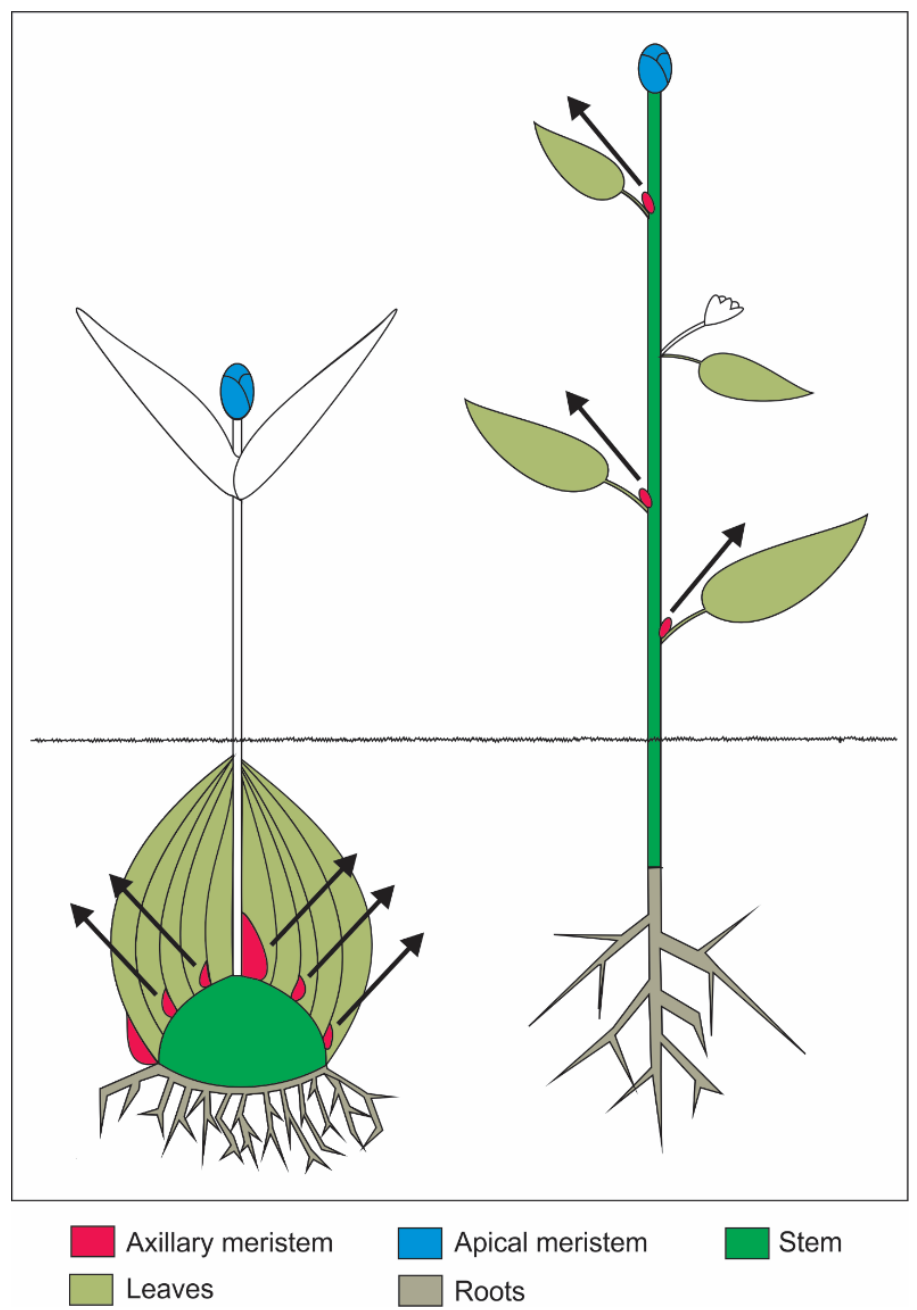

Figure 1. Architecture of a bulbous and a non-bulbous plant. (A) Tulip (B) Model dicot plant. Initiation of axillary meristems takes place in the axils of bulb-scales (A) or leaves (B). They form a bud like structure and undergo a period of dormancy. Once bud dormancy is broken, axillary buds grow out and develop into daughter bulbs in bulbous plants, or axillary branches in a typical dicot plant. In tulip, normally only two of the axillary buds will develop into daughter bulbs and once the apical bud blooms and dies, the closest axillary bud will become the apical bud for the next season. In bulbous plants the stem is called basal plate and it is a modified stem; Bulb-scales of bulbous plants are modified leaves. Arrows represent axillary bud outgrowth.

This control is mediated by a balanced hormonal signalling between auxin, cytokinin and the recently discovered strigolactone (Kebrom et al. 2013). Evidence for a role of strigolactone in 
axillary bud outgrowth is given by ramosus (rms) mutants in pea, decreased apical dominance (dad) in petunia, more axillary growth (max) in Arabidopsis, and dwarf (d) or high tillering dwarf (hdt) in rice (Booker et al. 2005; Morris et al. 2001; Napoli 1996; Ishikawa et al. 2005; Liu et al. 2009) In Arabidopsis MAX1, MAX3 and MAX4 are involved in strigolactone biosynthesis while MAX2 plays a role in strigolactone signalling. Although the exact crosstalk between auxin, strigolactone and cytokinins in the control of shoot branching is not yet entirely understood, it is clear that auxin and strigolactone inhibit bud outgrowth while cytokinins promote it. In this system, a bud-specific gene that promotes bud arrest could be the key element to integrate the bud outgrowth pathway. Indeed, such a gene exist and is represented by Teosinte branched 1 (TBI) in maize and BRANCHED (BRC1) in Arabidopsis (dicot). TBI was first identified in maize and appears to encode for a transcription factor from the TCP family (Aguilar-Martínez et al. 2007). Evidence in Arabidopsis and pea show that the TBI ortholog $B R C 1$ is up-regulated by strigolactone and down-regulated by cytokinins (Aguilar-Martínez et al. 2007; Braun et al. 2012b). A more recent study supports the idea of BRCl as a second messenger to induce and maintain bud arrest by negatively regulation of cell cycle, ribosome translation, and promotion of Abscisic Acid (ABA) signalling (González-Grandío et al. 2013). Because, outgrowth of axillary buds seems to be the major limiting factor in vegetative propagation of bulbs, the strigolactone signalling pathway and TBl-like genes are first targets of choice to study and optimize vegetative propagation in these plant species.

\section{Flowering time control and flowering induction}

Besides branching and axillary bud development, flowering time is an important trait influencing reproduction capacity in bulbous species. Plants are continuously sensing their environment, for being in the reproductive phase under optimal conditions and securing their reproductive success. Besides environmental cues, such as photoperiod and temperature, flowering time is also controlled by endogenous signals, including hormone levels and plant age (Lang 1952). In the model plant Arabidopsis the vegetative phase transition and floral induction, are well studied at the molecular level and the complex gene regulatory networks underlying these processes have recently been reviewed (Andres and Coupland 2012; Srikanth and Schmid 2011). We will discuss flowering time control here only briefly, with a focus on the pathways that are the most important for flowering in most of the bulbous species (Fig. 2), which are the aging and temperature pathways. The juvenile vegetative phase (aging pathway) can take up to seven years in bulbous species. Upon reaching the adult vegetative stage, the transition to reproductive development can be induced, which in tulip e.g. is triggered by relative warm temperatures in the spring or early summer (ambient temperature pathway). However, for development of the floral meristem into a complete flower and for elongation of the floral stem, a prolonged period of cold is essential (dormancy release), in analogy with bud dormancy release in trees (Cooke et al. 2012). 


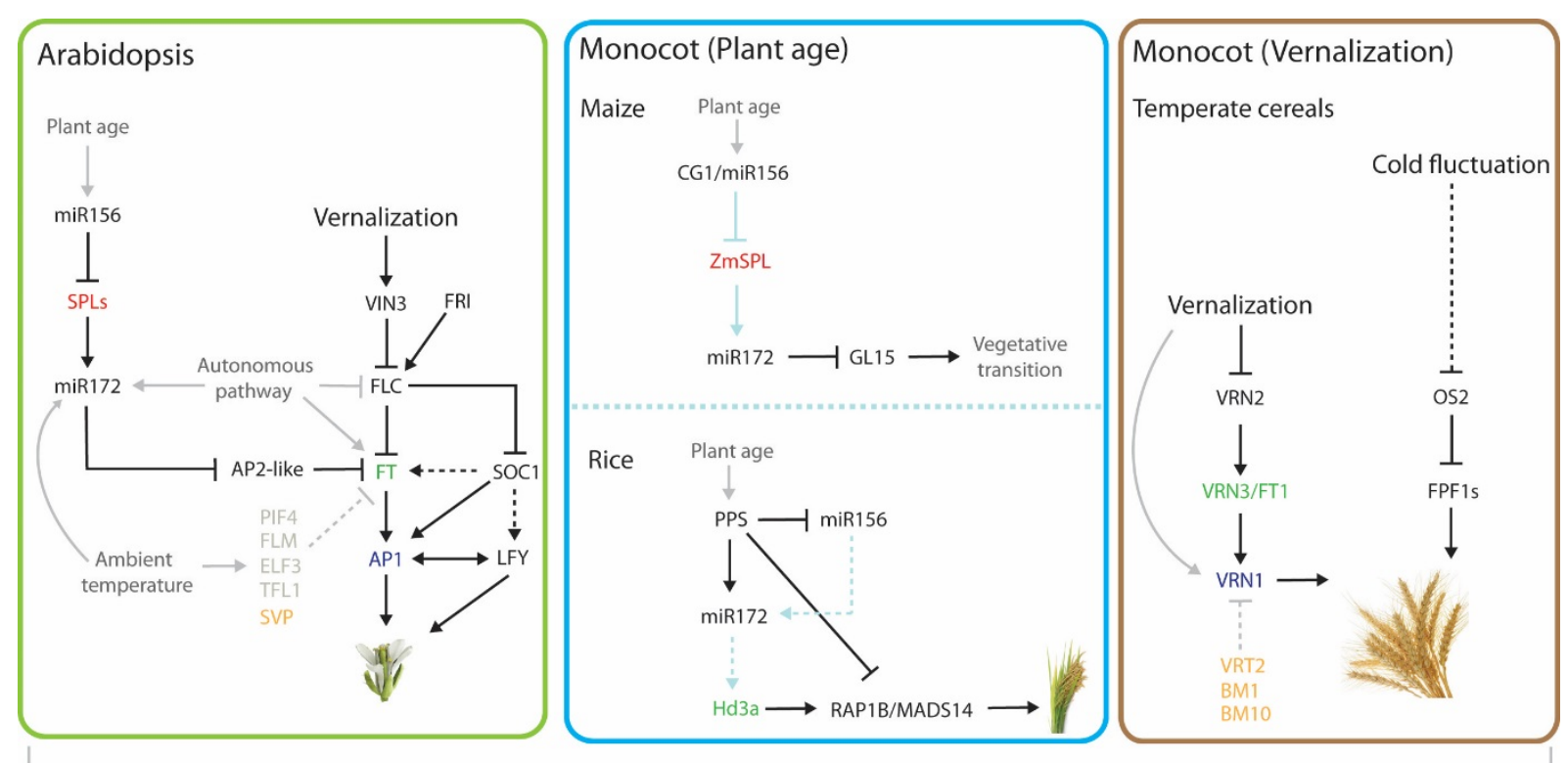

Figure 2. Comparison of the gene regulatory networks for flowering time control in dicots and monocots. In Arabidopsis (A) upon aging miR156 is repressed leading to the up-regulation of selected SPL genes which promote the vegetative phase transition (aging pathway). External cues, e.g. ambient temperature, trigger the activation of miR 172 via the SPL genes leading to the repression of the floral repressors AP2 and AP2-like genes. In order to be able to flower, winter annual Arabidopsis ecotypes first needs a pro-longed period of cold leading to the activation of VIN3 and repression of FLC. Ultimately, this results in the activation of the floral integrators FT and SOCl, followed by the activation of the floral meristem identity genes API and LFY. In the monocot maize ( $B$, top), the vegetative phase transition is regulated by the suppression of the AP2-like gene GLOSSY15 (GL15) through the activation of miR172. CORNGRASS1 (CG1) encodes miR156 and similar to Arabidopsis, might represses $\mathrm{ZmSPL}$ leading to the activation of miR 172. In the monocot rice (B, bottom), PETER PAN SYNDROME (PPS) is involved in the repression of miR156 and the activation of miR 172. This might occur directly by PPS or indirectly (dotted blue arrow) through miR 156. Upon unfavourable environmental conditions, PPS represses RAPIB/MADS14, independent of Hd3a (rice FT homolog). In monocot temperature cereals (C) the FT homolog VRN3/FT activates VRN1 upon a prolonged period of cold, leading to flowering. Nevertheless, the SVP homolog VRT2 represses VRN1. Shorter periods of cold repress OS2 which inhibits stem elongation through FPFls. Taking into account this knowledge from model species and assuming general conservation of the gene regulatory networks, a putative flowering controlling network can be designed for bulbous species. Genes with similar kind of functions in the different species are marked with the same colour.

Plant age is one of the endogenous factors that can be linked with developmental phase transitions and competence of the shoot apical meristem for environmental signals triggering flowering. The age-dependent vegetative transition in Arabidopsis is regulated by microRNA 156 (miR 156) and the SQUAMOSA PROMOTOR BINDING PROTEIN-LIKE (SPL) genes that are targeted by this microRNA. The repression of miR 156 results in up-regulation of several SPL genes which promotes vegetative transition (Fornara and Coupland 2009). Two recently published studies showed that miR 156 levels are responding to sugars (Yang et al. 2013; Yu et al. 2013). Whereas a bulb is a storage organ and it is well known that sugars get re-located towards the shoot apical meristem and stem (sinks) upon flowering-inducing temperature changes (Lambrechts et al. 1994), it will be of interest to focus on this particular pathway in the hunt for signalling components involved in flowering time control of bulbous species. 
After the switch from the juvenile to the adult vegetative phase, the plant becomes competent for flowering inducing external cues. Furthermore, reproductive development is triggered by the activation of microRNA172 (miR 172) by the SPL genes, which results in the repression of a set of APETALA2 (AP2)-like genes, acting as repressors of flowering (Zhu and Helliwell 201 1). Both microRNAs miR 156 and miR 172 are conserved in dicots and monocots (Axtell et al. 2007). Although, the age dependent phase transition is studied to a lesser extent in monocots (Strable et al. 2008; Tanaka et al. 2011) (Fig. 2), performed experiments reveal a high level of conservation in the regulatory mechanisms controlling flowering time in between different species.

Vernalization is the requirement for a period of prolonged cold to overcome a block on flowering in winter annual plants. In Arabidopsis FLOWERING LOCUS C (FLC) is the key floral repressor in this process, and this transcription factor was shown to act as a direct transcriptional repressor of the so-called floral integrator genes FT (FLOWERING LOCUS T) and SUPPRESSION OF OVEREXPRESSION OF CONSTANSI (SOC1) (Fig. 2). FLC is activated by the positive regulator FRIGIDA (FRI) that acts in a large multi-protein complex. During winter, the transcriptional regulator VERNALIZATION INSENSITIVE3 (VIN3) will respond to a prolonged period of cold, resulting in its gradual activation. As a consequence $F L C$ will be repressed providing the shoot apical meristem competence for floral inducing cues, such as optimal temperatures and appropriate photoperiod conditions (Choi et al. 2011). In monocots however, FLC-like genes could not been identified. In wheat a different gene, VERNALIZATION2 (VRN2), encoding for a Zinc finger-CCT domain containing transcription factor (Yan et al. 2004), is down-regulated by vernalization. This repression results in the activation of the FT homolog VERNALIZATION3 (VRN3)/FLOWERING LOCUS Tl (FTI) and the APETALAl-like VERNALIZATIONI (VRNI) gene during a period of prolonged cold (Alonso-Peral et al. 2011; Yan et al. 2006). Three genes homologous to the Arabidopsis SVP gene; VRT2, BMI and BM10 respectively, are able to repress VRNI but their role in vernalization or floral transition is not completely understood (Trevaskis et al. 2007; Kane et al. 2005). Besides a pro-longed period of cold (vernalization response), short cold stresses repress the grass specific MADS box gene ODDSOC2 (OS2). A proposition was made that OS2 is present in a pathway that delays the transition to reproductive development and that additionally inhibits stem elongation (Greenup et al. 2010). Altogether, this suggests that the vernalization response has evolved independently in monocot and dicot plants, although members from the MADS box transcription factor family play an important role in both. Bulbous plants, such as tulip, also require a prolonged period of cold. Though, in this case it is not essential for the meristematic switch from vegetative to reproductive development, but to release dormancy in the already existing floral bud and to induce stretching of the floral stem. Despite that this dormancy release is different from the vernalization response, more and more evidence is provided that the underlying regulatory mechanisms are comparable (Horvath 2009). In addition to the vernalization response, flowering time also depends on 
relative small fluctuations in ambient temperatures. Genes involved in flowering time control and responding to changes in ambient temperature are e.g. FLOWERING LOCUS M(FLM)/MADS AFFECTING FLOWERINGI (MAFl), SHORT VEGETATIVE PHASE (SVP), EARLY FLOWERING3 (ELF3), TERMINAL FLOWERI (TFL1) and PHYTOCHROME INTERACTING FACTOR4 (PIF4) (Balasubramanian and Weigel 2006; Kumar et al. 2012). Nevertheless, in contrast to the wealth of knowledge on the vernalization pathway, insight in the gene regulatory network underlying the ambient temperature pathway is just emerging.

\section{Ways to transfer knowledge from model plants to economically important crop species}

To transfer the wealth of knowledge gained from studies in model species towards crops and e.g. bulbous plant species, diverse roads can be taken. According to the methodology used to link the molecular basis of life (e.g. genes) with biological functions, such methods can be divided in bottom-up or top-down approaches (Fig. 3). The former one uses deductive reasoning, meaning that the knowledge is built from the constitutive parts (e.g. genes) to the systems, while top-down requires inductive reasoning: from systems to causal genes.

\section{Bottom-up approach}

The start point of this approach is the identification of putative orthologues genes in crops for genes of interest in model species (Salentijn et al. 2007). In general, this is based on sequence homology and the assumption that the molecular pathways underlying the control of the biological processes, and hence the involved genes, are conserved. A widely used method in the past was the identification of highly homologous genes by genomic or cDNA library screenings (e.g. (Xu et al. 1995; Sun et al. 1999)). For this purpose hybridization can be applied or alternatively PCR-based methods, using degenerated oligonucleotides. Once the unknown target genes are identified, they can be sequenced and subsequently compared in silico with the gene sequences from the model species. An advantage of this method is that construction of such libraries does not require a priori genome sequence information. However, to date there are only limited comprehensive genomic libraries available for flower bulb species, likely due to the complex genome sizes. When sequence information is available for the species of interest, identification of homologues is normally done in silico via BLAST-based sequence alignments (Altschul et al. 1990). However, there are limitations to the above discussed simplistic approaches, given by the fact that sequence similarity does not always imply functional similarity. This is nicely exemplified by differences in function for key genes in the vernalization pathway between monocots and dicots (e.g. API-like genes; Fig. 2). Furthermore, large-scale evolutionary events such as duplications can cause functional divergence for paralogues genes. When evolutionary events are taken into account, comparative studies, such as synteny mapping can provide information on orthology of the blasted sequences (McCouch 2001). 
Whereas in the past, this was restricted to species for which the genome was sequenced or for which a detailed genetic map was available, integrating high-throughput Next Generation Sequencing (NGS) data makes it possible to apply this type of studies to crops that lack a reference genome sequence (Galvão et al. 2012) and hence, make it also possible to use synteny mapping for bulbous plant species in the near future. Regardless whether orthology will be taken into account, various experimental tools can be applied to guide the identification of genes or proteins with identical functions based on intrinsic characteristics of the molecules, such as protein-protein interaction capacity or their specific expression patterns.

\section{Top-down approach}

Top-down methodologies build vast amounts of high-throughput data in order to establish systems from which identifying causal genes would be feasible (Fig. 3). Large scale phenotyping platforms coupled to linkage mapping, and gene expression-based analyses, such as the generation of Expressed Sequenced Tags (EST) or genome-wide transcriptome profiling via microarray analyses or RNA-seq, are examples of sources for such large-scale data sets. EST datasets are a rich source for designing custom-made DNA microarrays (Lorenz et al. 2003), but for many species of interest there are no sufficient datasets available to create a proper microarray platform. In that case, cross-species microarrays, in which probe sequences are derived from a model species and hybridization is performed with material from a crop of interest, is an attractive alternative to profile expression patterns (e.g. (Moore et al. 2005; Wang et al. 2010)). However results have to be interpreted carefully because of variance in efficiency of probe-transcript hybridization, caused by differences in sequence similarities or e.g. number of gene copies, due to species-specific duplication events (Lu et al. 2009). Unlike classical microarray experiments, RNA-seq does not require genome sequence information (Wang et al. 2009), neither a priori knowledge of gene functions. Furthermore, the method is highly sensitive and accurate providing detailed insight in gene transcription levels, as well as splicing variants across different physiological or morphological samples.

Together, these characteristics make this technology an ideal tool to gain insight in the transcriptome of bulbous plants and to study differential gene expression for relevant biological process in these species. Nevertheless, assembling the enormous amount of short reads produced by RNA-seq is a bioinformatics challenge (Martin and Wang 2011); especially for crops that lack a reference genome, which is the case for many economically important crops 


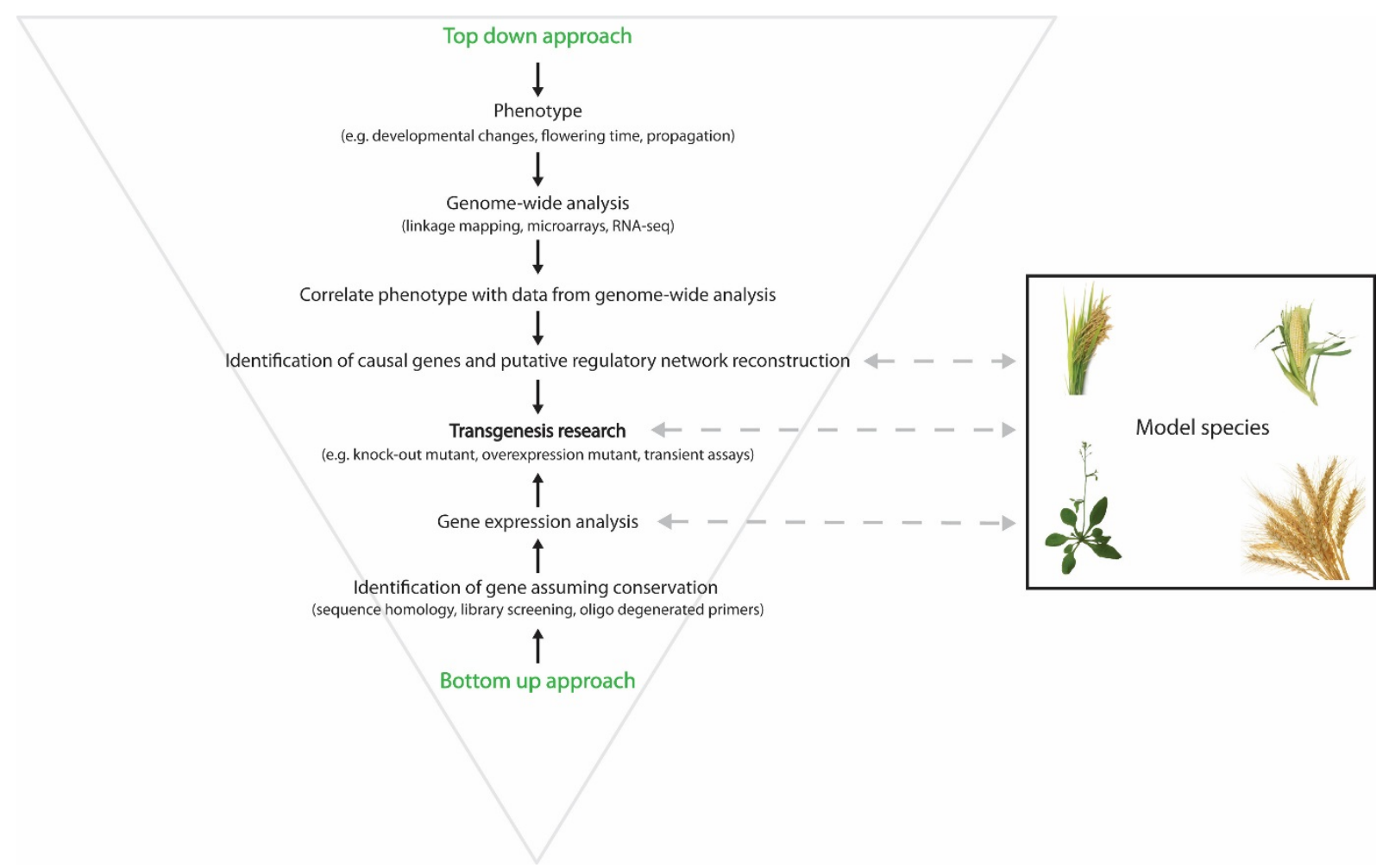

Figure 3. Flow chart of how knowledge can be transferred from model species to economically important crops. Both bottom-up and top-down approaches are indicated and come together at the level of functional confirmation of candidate gene functions by transgenic research.

and in particular for bulbous plants. In absence of a reference genome, de novo transcriptome assembly is used as first approach (Garber et al. 2011). A successful example of the latter approach, was recently presented for grapes, that like bulbous species preferably sustain through vegetative propagation (Venturini et al. 2013). Besides transcriptomics data, information from other "omics" types of approaches can be implemented. Currently, after transcriptomics the proteomics field is the most advanced and detailed quantitative information can be obtained at the protein level (Bindschedler and Cramer 2011; Kaufmann et al. 2011). Also metabolomics is improving, but generated datasets are more fragmented and improvements of both throughput and reproducibility are needed (Saito and Matsuda 2010).

The next step for all above mentioned top-down approaches, aiming to obtain information on gene activity and intrinsic gene product characteristics at a genome-wide scale, is the identification of genes or sets of genes that behave in a manner associated to the biological process of interest. Subsequently, potential gene regulatory networks can be reconstructed based on this information, which can be compared to and fed back to knowledge from model species (Fig. 3). In this respect it is good to realize that for the usage of e.g. metabolomics data an additional hurdle needs to be taken in correlating metabolite concentrations to e.g. gene expression patterns and finally gene functions. 


\section{Verification of gene function}

Both bottom-up and top-down approaches give a selection of genes that are potential key players in the biological process under study, and for which preferably the function should be validated. In Arabidopsis this is usually done through the selection of loss-of-function mutations in collections of T-DNA insertion plants (Slater et al. 2003). Alternatively, stable transformants can be generated or functions can be investigated based on transient expression assays by agro-infiltration or virus induced gene silencing (VIGS) (Lu et al. 2003; Yang et al. 2000). The majority of methods that are available today for gene function verification depend on transgenic approaches. Despite that these technologies are already available for thirty years and have undergone various improvements over the last decades, it is still far from trivial to transform any desired plant species. Therefore, it is still common practise to perform gene function verifications by overexpression or complementation studies using a model species as target (cross-species analysis) (Li et al. 2013; Tsaftaris et al. 2012).

\section{Examples of successful knowledge transfer to bulbous plants}

\section{Bottom-up "gene-by-gene" approach}

Several of the above discussed methods to transfer knowledge from model species to crops have been used already in bulbous species. Probably one of the best known examples of the bottom-up approach is related to the specification of floral organ identities by MADS box transcription factor genes according to the ABC-model (Litt and Kramer 2010; Rijpkema et al. 2010; Ferrario et al. 2004). Floral organs in higher eudicots are organized in four concentric whorls, with sepals in the outer whorl, petals in whorl two, stamens in whorl three and carpels in the inner fourth whorl. The classical $A B C$ model predicts the establishment of the four floral organ identities by the combinatorial action of MADS domain transcription factors and the accessory gene regulatory network appeared to be highly conserved. Based on the assumption that this network will also be conserved in bulbous flowers, hypotheses were generated to explain particular flower mutants in these species. Classical examples are the so called 'double flowers', in which stamens are converted into petals or petaloid organs, which in theory can be caused by alterations in B- or C-class MADS box genes. Expression studies in the double-flowered lily "Elodie" provided evidence that this phenotype indeed was caused by the miss-expression of the putative Lily C-class gene LelAG 1 (Akita et al. 2008). Besides the $C$ class gene, a putative A class (API-like) and other MADS box genes of the C/D class have been identified in Lilium longiflorum (Chen et al. 2008; Tzeng and Yang 2001). Also in Crocus sativus a putative API gene was identified as well as a SEPALLATA3 (SEP3)-like gene from the E-class (Tsaftaris et al. 2004; Tsaftaris et al. 2011). Despite strong conservations in flower organisation, plants belonging to the Liliaceae family have in general a slightly modified flower structure with two almost identical outer floral whorls, known as tepals. Based on this 
phenomenon a modified $A B C$ model was proposed (van Tunen et al. 1993), suggesting that class B genes are also expressed in whorl one, leading to the same petaloid identity in the outer two whorls. The putative class B genes from Tulipa gesneriana were cloned and characterized (Kanno et al. 2003). In agreement with the hypothesized alternative model, the two DEFICIENS (DEF)-like genes TGDEFA and TGDEFB as well as one GLOBOSA (GLO)-like B-type gene TGGLO, were found to be all expressed in whorls one, two and three. The same model is also supported by the identification and analysis of B-class floral homeotic gene PISTILLATA (PI)/GLO in Crocus sativus (Kalivas et al. 2007). All together, these examples show the power of a "gene-by-gene" bottom-up approach in case of well-studied and strongly conserved biological processes.

\section{Top-down "transcriptome profiling" approach}

Performing large-scale expression studies coupled to phenotyping is an advanced technology to identify key genes involved in a particular biological process. In lily e.g., a custom-made cDNA microarray was designed and generated, consisting of several cDNA's obtained from different pollen-related tissues (Huang et al. 2006). Following, a differentially expressed gene was identified encoding for a putative protein containing ankyrin repeats and a RING zincfinger domain, named LIANK. Comparison of LIANK to functionally characterized genes in model plants suggested ubiquitin ligase activity for the gene product. Further experiments could confirm this molecular function and revealed an important role for this gene in polar pollen tube growth, showing the relevance of the followed approach. Despite the potential of this method and the large number of examples of success stories in a variety of food crops, the approach has been hardly explored in bulbous plant species.

\section{Gene function verification using model species}

Upon the identification of functional analogues genes, verification of the function is an important process. Monocots are known to be recalcitrant to Agrobacterium-mediated transformation and therefore most of the flower bulb transformations have been achieved through gene-gun techniques (e.g. (De Villiers et al. 2000; Kamo et al. 1995; Watad et al. 1998)). However, a major drawback of gene-gun transformation over Agrobacterium-mediated transformation is the lack of stable integrations on one hand and the unintended, but frequently observed integration of multiple gene copies in the case of a successful integration on the other hand. The latter can be a trigger for undesirable recombination events, genomic rearrangement, or silencing of the transgene (Hooykaas and Schilperoort 1992). Conveniently, evidence has been provided for the presence of certain Agrobacterium strains being able to infect flower bulb species such as Ornithogalum (Van Emmenes et al. 2008), Gladiolus (Kamo et al. 1995) and Lilium (Cohen and Meredith 1992). More recently Li and collaborators proved that insertion and stable integration of $\mathrm{Zm401}$ gene in Lilium is possible via Agrobacteriummediated transformation, which opens the door for more transgenic efforts in flower bulbs (Li 
et al. 2008). Nevertheless, in general transformation of bulbous plants is tedious and stable transformation frequencies are low (Wang et al. 2012; Lu et al. 2007).

Therefore, heterologous complementation studies in Arabidopsis are widely used as an alternative to verify the function of a candidate gene found in bulbous species. For example, a homolog of CENTRORADIALIS (CEN)/TERMINAL FLOWER (TFL), CsatCEN/TFL 1 respectively, was cloned from Crocus sativus and functionally characterized in Arabidopsis. In Arabidopsis, TFL controls axillary meristem identity, inflorescence development and flowering time (Alvarez et al. 1992). Overexpression of CsatCEN/TFL1 in a tfll Arabidopsis mutant background resulted in complementation of the mutant phenotype, indicating that the gene isolated from C. sativus is able to function as TFL1 (Tsaftaris et al. 2012). A similar study revealed that a FT-like gene in Narcissus tazetta var. chinensis, known as NFT1, act as a flowering time regulator when ectopically and constitutively expressed in ft-3 mutant Arabidopsis plants. In these transgenic lines, SOCl a target of FT showed to be up-regulated as expected based on FT functioning in Arabidopsis (Li et al. 2013).

Besides stable transformation, transient technologies, such as VIGS, have been applied in bulbous species. A fragment of a putative PDS gene supposed to encoding phytoene desaturase, which is involved in carotenoid metabolism and photosynthesis, has e.g. been derived from lily and caused a bleaching phenotype in N. benthamiana after infiltration (VIGS). This phenotype was expected, because it is known that silencing of PDS results in photo bleaching symptoms caused by a decrease in leaf carotene. This reveals that genes of monocot species can be used to silence their counterparts in the dicot $N$. benthamiana regardless of their distant evolutionary relationship (Benedito et al. 2004; Wang et al. 2009) and providing hints for possible functions of the used genes.

Although the above mentioned examples show the success and power of heterologous functional analyses based on stable or transient transformation, it is good to realize that these type of experiments do in principle not indicate more than that a gene from a crop has sufficient sequence homology and overlap in functional domains to take over the activity of the endogenous gene in the model system. Consequently, this is no guarantee that a similar function can be assigned to the identified gene in the crop species. Difference in the spatial or temporal expression pattern might already withhold the gene from its supposed function based on the heterologous functional analysis.

\section{Future directions and challenges}

So far most molecular-oriented research studies in recalcitrant crops and bulbous plants have focussed on the identification of a single candidate gene. Analyses of complete regulatory pathways, as is nowadays common in model species, are hardly done yet. However, with the speed NGS technologies are developing (Schneeberger and Weigel 2011), molecular 
technologies become attractive tools to analyse important biological processes in non-model species. Shahin and colleagues (2012) provided e.g. the first transcriptome dataset of lily and tulip by sequencing of ESTs with the 454 NGS technology (Roche; http://www.454.com/). Comparative genomics helped with the search for gene conservation between tulip and lily, and the contigs could be annotated on the basis of the rice genome annotation (Sequencing Project International Rice, 2005). Subsequently, molecular function, biological process and cell component were predicted for the identified genes that all together resemble about $40 \%$ of the lily and tulip transcriptome.

Hence, this approach provides fast insight in the active part of bulbous plants genomes, with a limited investment and avoiding the need for deciphering the complete genome sequence, which in the case of tulip is 200 times the size of the Arabidopsis genome. Although this is a great step forward, the authors realized and emphasized that deeper sequencing and analysis of time series for various tissues or cell types is essential to obtain sufficient information for extended comparative and functional gene studies. Furthermore, traditional sequencing techniques were producing long contiguous DNA sequence reads up to $1 \mathrm{~kb}$ in length; however, the majority of the latest introduced NGS platforms generate huge quantities of short sequence tags (50 to 100bp), requiring sophisticated assembly algorithms and bioinformatics solutions (Reviewed in: (Nagarajan and Pop 2013).

Besides tackling this problem by a bioinformatics approach, technical improvements such as paired-end sequencing, helps to solve the assembly problem. Additionally, output from different platforms (e.g. PacBio; http://www.pacificbiosciences.com/) can be incorporated to overcome this problem to a certain extend. Nevertheless, the biggest barrier in this type of research will not be the generation of large scale data sets and the identification of complete gene sequences, but to extract the genes and alleles of importance for the process under study; or in other words, to find the needle in the haystack. In this respect it is good to take into account that the success rate of RNA-seq experiments for gaining knowledge in a particular biological process strongly depends on a well-defined research question, followed by detailed temporal and spatial differential expression analyses (Van Verk et al. 2013). In addition to the correct input of biological material and the usage of optimal algorithms to extract genomewide differential gene expression patterns, it is of utmost importance to improve the methods for the annotation of the identified genes.

As discussed above, simple blast-based alignments are a good starting point, but in the case when no or only low homology exist with known gene sequences, other technologies are essential. Recently, bioinformatics and systems biology tools have been developed for this purpose, in which e.g. domain co-occurrence networks are generated (Wang et al. 2013) or information from various data sources or prediction programs is combined (Kourmpetis et al. 2011). 
Despite the importance of bulbous plants for the ornamental industry, these species remained under investigated at the genetic and molecular level. However, thanks to the latest developments in transgenic research, the "omics" area, and in the field of systems biology, the detailed study of flowering and vegetative propagation in bulbous plants, resembling the two most important biological processes for agronomical improvements, comes in sight. From a breeders' perspective, shortening of the juvenile phase will help increasing the speed of selection processes for new varieties, with e.g. improved bulb productivity, ornamental characteristics and pathogen resistance. Hopefully, these developments will keep this sector flourishing in the coming century.

\section{Acknowledgements}

We would like to thank Wilco Ligterink for critically reading of the manuscript and useful suggestions. Financial support for our research is provided by the Product Board of Horticulture (PT), TTI-Green Genetics, the Dutch Ministry of Economic Affairs, and the Royal General Association for Bulb Culture (KAVB). 



\section{CHAPTER 3}

\section{Elucidating and mining the Tulipa and Lilium transcriptomes}

Natalia M. Moreno-Pachon\#, Hendrika A.C.F. Leeggangers\#, Harm Nijveen\#, Edouard Severing, Henk Hilhorst, Richard G.H. Immink \#Both authors contributed equally to this work. 


\begin{abstract}
Genome sequencing remains a challenge for species with large and complex genomes containing extensive repetitive sequences, of which the bulbous and monocotyledonous plants tulip and lily are examples. In such a case, sequencing of only the active part of the genome, represented by the transcriptome, is a good alternative to obtain information about gene content. In this study we aimed to generate a high quality transcriptome of tulip and lily and to make this data available as an open-access resource via a user-friendly web-based interface. The Illumina HiSeq 2000 platform was applied and transcribed RNA was sequenced from a collection of different lily and tulip tissues, respectively. In order to obtain good transcriptome coverage and to facilitate effective data mining, assembly was done using different filtering parameters for clearing out contamination and noise of the RNA-seq datasets. This analysis revealed limitations of commonly applied methods and parameter settings used in de-novo transcriptome assembly. The final created transcriptomes are publicly available via a user friendly Transcriptome browser (http://www.bioinformatics.nl/bulbs/db/species/index). The usefulness of this resource has been exemplified by a search for all potential transcription factors in lily and tulip, with special focus on the TCP transcription factor family. This analysis and other quality parameters point out the quality of the transcriptomes, which can serve as a basis for further genomics studies in lily, tulip, and bulbous plants in general.
\end{abstract}

Key words: Tulip (Tulip sp), lily (Lilium sp), genes, transcriptome browser, sequencing, de novo assembly 


\section{Introduction}

Modern sequencing technology, also referred to as Next Generation Sequencing (NGS), quickly generates large amounts of sequence data at lower cost in comparison with traditional Sanger sequencing (Marguerat and Bähler 2010) (Schatz et al. 2010). While sequencing and assembly of large genomes still represent a technical challenge and a laborious procedure (Treangen and Salzberg 2012), sequencing the expressed part of the genome, represented by the transcriptome, is nowadays achievable and can level down the complexity and provide useful information (Riesgo et al. 2012). Therefore, transcriptome sequencing may represent an alternative to whole genome sequencing for species with large complex genomes when the aim is to generate a comprehensive database of genomic resources, suitable for gene identification, allele mining, or genome wide expression studies (Hou et al. 201 1; Liv et al. 2012; Duangjit et al. 2013).

Bulbous plants, also classified as geophytes, represent species with economic relevance, large genomes and relatively scarce genomic resources. In short, geophytes are plants with storage organs and renewal buds resting in underground structures (Kamenetsky and Okubo 2013) (Fig. 1). Tulip and lily (Tulipa sp and Lilium sp) are ornamental geophytes with an estimated genome size of 25 and $36 \mathrm{~GB}$, respectively (Shahin et al. 2012b). One of the first studies of a transcriptome characterization for both species was done by Shahin et al. in 2012 using 454 pyro-sequencing technology of messenger RNA (mRNA) from leaves (Shahin et al. 2012b). They obtained 81,791 unigenes for tulip with an average length of 514 bp and 52,172 unigenes for lily with an average length of $555 \mathrm{bp}$. Later studies have e.g. focused on sequencing the transcriptome of leaves (Wang et al. 2014), bulblets (Li et al. 2014b) and meristem-enriched tissue (Villacorta-Martin et al. 2015) of different Lilium cultivars, using the Illumina HiSeq sequencing platform. These studies resulted in the identification of 37,843 unigenes for leaves (Wang et al. 2014), 52,901 unigenes in bulblets (Li et al. 2014b) and 42,430 genes for the meristem-enriched lily tissues (VillacortaMartin et al. 2015).

Despite continuous efforts to broaden the genetic resources of the bulbous species tulip and lily, characterization of their entire transcriptome is far from being completed. The information generated to date only covered leaf and meristem-enriched tissues and, furthermore, the data is difficult to access and mine for non-bioinformaticians. Our study aimed to generate a high quality and extensive transcriptome of these two bulbous species and making this valuable resource publicly available through a user-friendly and freely accessible web-based interphase, allowing easy data mining. The Illumina HiSeq platform was used to sequence a pooled sample for lily and for tulip, each made up of a mixture of equal amounts of poly adenylated mRNA obtained from flowers, stem, leaves, bulb and bulblets. Even though short reads are generated with the Illumina HiSeq platform, a tremendous throughput can be reached, resulting in an improved coverage of rare transcripts in comparison to the other 


\section{Chapter 3}

platforms used in some of the previous transcriptome studies of bulbous species (Shahin et al. 2012b; Kamenetsky et al. 2015).

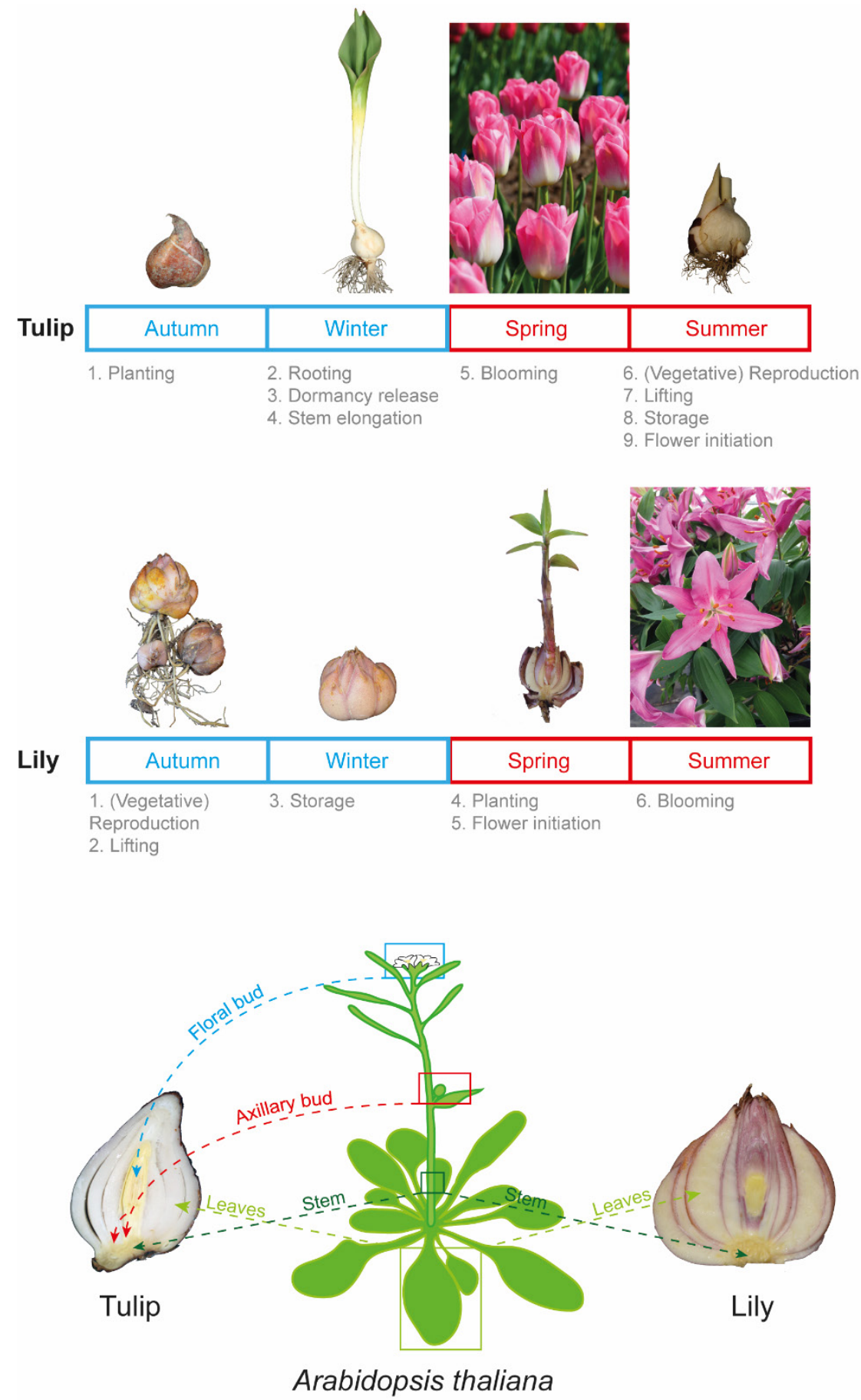

Figure 1. Life cycle and architecture of tulip and lily bulbs. (A) Tulip and lily yearly growth cycle. Note that their growth cycle is very similar. Both require a period of cold, but for different purposes and blooming occurs in different seasons. (B) Bulbs can be regarded as modified plants where the stem has shortened into a basal plate, the leaves have been modified into bulb-scales. In the tulip bulb the axillary buds are located in the axils of the bulb-scales and the floral bud is located in the center on top of the basal plate. 
The generated data was used to assemble reference transcriptomes for tulip and lily. For this purpose, different assembly settings were explored, aiming to generate an optimal transcriptome for gene mining. To proof the quality of the generated data sets, a comparison was made between the transcripts found in the bulbous species tulip and lily and the genes of the model species Arabidopsis thaliana and Oryza sativa (rice). In addition, we searched for potential transcription factors present in both transcriptomes and compared their distribution with the distribution of transcription factors in the model species Arabidopsis and O. sativa. Subsequently, a web-based interface (Kamei et al. 2016), which we call Transcriptome Browser, was implemented for data presentation and mining.

The various possibilities of this browser are exemplified by zooming-in on a particular plantspecific gene family and the identification of all potential members of this transcription factor family. This activity enlightens the usefulness of the tulip and lily transcriptome browser in mining high-throughput sequencing data and identifying sequence information from lowly expressed, but important regulatory genes. Furthermore, these analyses revealed the quality of our data set and show how this resource can be explored in the future to study biological processes in bulbous plants at the molecular level.

\section{Results}

\section{Transcriptome sequencing and assembly}

The Illumina HiSeq 2000 platform was used to sequence the tulip and lily transcriptome of a wide range of tissues varying from bulb scales to flowers. After trimming and removal of low quality reads, a similar number of paired end reads were obtained for both libraries: $169,920,574$ reads for tulip and 165,031,389 for lily. Subsequently, Trinity software (Grabherr et al. 201 1 b) was used to assemble both transcriptomes de novo and this assembly yielded to 499,780 transcripts for tulip and 569,305 for lily with an average length of $561 \mathrm{bp}$ and $487 \mathrm{bp}$, respectively. When not taking the isoforms into account and without applying additional data filtering, Trinity predicted 380,091 genes for tulip and 467,241 for lily (Table 1). Transcript over-estimation is common in de novo sequencing studies because the lack of a reference transcriptome or genome limits the assembly of sequences that represent non-overlapping pieces of the same gene. Transcripts expressed at extremely low levels can also cause noise because they may not be reliably assembled (http://cole-trapnell-lab.github.io/cufflinks/cufflinks/). Furthermore, it is difficult to distinguish between isoforms of one gene versus the existence of more gene copies as a consequence of duplications (Chang et al. 2015).

Therefore, filtering out lowly expressed transcripts is a routine procedure applied during transcriptome assembly to get rid of noise and contamination, and it yields, in general, significantly reduced numbers of predicted transcripts and genes. To compare and find the optimal parameters for our two datasets, but retaining the full complexity of the tulip and lily 
transcriptomes, we generated three additional assemblies based on different abundance filtering settings. The three new assemblies consisted of transcripts with equal or more than 10 or 20 counts; and transcripts occurring at least more than once per million (TPM), respectively. As summarized in Table 1, increasing the cut-off value to filter out transcripts with low abundance leads to a dramatic decrease in the number of predicted transcripts and genes, but improves the N50 and average transcript length.

Table 1. Summary statistics of the tulip and lily transcriptomes generated by non-filtered data and upon applying three different filtering parameter settings.

\begin{tabular}{|c|c|c|c|c|c|c|c|c|}
\hline & \multicolumn{2}{|c|}{ Non-filtered } & \multirow{2}{*}{$\begin{array}{l}\text { Counts } \\
\geq 10 \\
\text { Tulip }\end{array}$} & per transcript & \multirow{2}{*}{$\begin{array}{l}\text { Counts } \\
\geq 20 \\
\text { Tulin }\end{array}$} & per transcript & \multicolumn{2}{|l|}{$T P M \geq 1$} \\
\hline & Tulip & Lily & & Lily & & Lily & Tulip & Lily \\
\hline Contigs & 499,780 & 569,305 & 174,442 & 252,040 & 112,256 & 131,912 & 39,171 & 38,688 \\
\hline Genes & 380,091 & 467,241 & 115,167 & 198,613 & 70,634 & 94,283 & 29,523 & 29,188 \\
\hline GC \% & 42,74 & 41,79 & 43,62 & 42,1 & 43,98 & 42,64 & 45,4 & 45 \\
\hline N50 & 695 & 514 & 1226 & 913 & 1478 & 1322 & 1573 & 1717 \\
\hline $\begin{array}{l}\text { Average } \\
\text { length }\end{array}$ & 561 & 487 & 933 & 703 & 1,139 & 989 & 1,017 & 1,035 \\
\hline
\end{tabular}

TPM: transcripts per million

The number of obtained transcripts and predicted genes, in combination with the average transcript length, is generally used as a quality indicator of de novo transcriptome assemblies. In an ideal situation, the number of predicted genes should be close to the number of genes expected for the species. Based on this criterion, using counts per transcript upward of 20 , seemed to be the best parameter since it reached a reasonable number of genes taking into account the number of genes found in sequenced plant genomes (e.g. rice (Sequencing ProjectInternational Rice 2005); Arabidopsis (Initiative 2000); poplar (Tuskan et al. 2006); loblolly pine (Neale et al. 2014)). Furthermore, this filtering resulted in a high average transcript length, suggesting a high percentage of complete and fully covered mRNA sequences in this assembly.

Nonetheless, it is important to realize that the high number of transcripts and predicted genes in the non-filtered transcriptome may not only be the result of miss-assemblies and non-plant contamination, but also because of the presence of incomplete or truncated rare, but valuable transcripts. Such incomplete transcripts may be the result of incomplete cDNA amplification, or mRNA degradation and breakage, and in general lowly expressed transcripts are more prone to be assembled as fragments due to limited sequencing coverage. To investigate this option in more detail, we studied-using the lily transcriptome as an examplehow filtering out lowly expressed transcripts affects the number of transcripts encoding plant 
orthologues as well as the transcripts considered to be contamination (Fig. 2). As expected, the three filtering options improved the raw transcriptome in terms of contamination, but surprisingly decreased also dramatically the number of plant orthologues retained. For example, TPM larger or equal to one reduced the contamination with almost $100 \%$ efficiency, but only retained a bit more than $20 \%$ of the plant orthologues from the non-filtered transcriptome database.

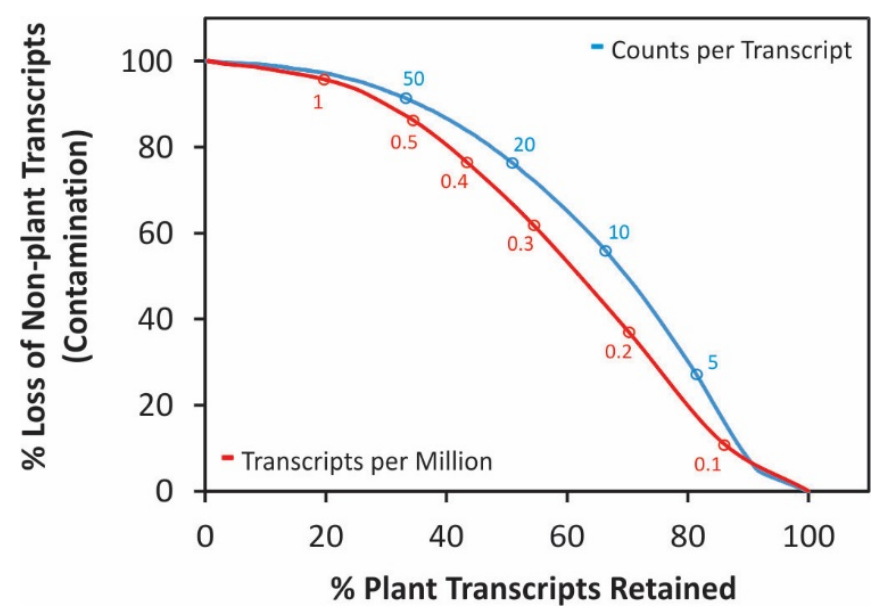

Figure 2. Effect of transcriptome filtering on the percentage of "non-plant" and plant transcripts retained. Filtering done based on counts per transcript.

This observation prompted us to gain more insight in the nature of the transcripts with low abundance. For this purpose, all removed transcripts per filtering method were compared with the Arabidopsis Information Resource (TAIR) database using the Basic Local Alignment Search Tool (BLAST). Within the removed transcript sequences many important gene products where present, e.g. encoding putative meristem signalling peptides (CLAVATA3/ESR) (Wang and Fiers 2010), which are known to be short in sequence and lowly expressed. Furthermore, transcript fragments of genes expected to be very locally and lowly expressed, such as some basic helixloop-helix (bHLH) transcription factors, wound-responsive protein-related and flowering promoting factors, were identified in these filtered-out transcript sets (see Supplemental Table 1). Hence, the use of a filtering method may lead to a transcriptome with improved quality based on average transcript length, but it results on the other hand in the removal of a substantial number of transcript fragments corresponding to important plant genes. Based on these observations, we decided to continue with a non-filtered transcriptome, including short, truncated, and incomplete transcripts, since this increases the chances of identifying sequence information of rarely expressed genes. In order to evaluate the completeness of these final assembled and selected tulip and lily non-filtered transcriptomes, Core Eukaryotic Genes Mapping Approach (CEGMA) analysis was used (Parra et al. 2007), showing that the generated transcriptomes of tulip and lily contain nearly $100 \%$ of the 248 core eukaryotic proteins ( $98.79 \%$ for both species). 


\section{Functional annotation}

TransDecoder 2.0.1 (Haas et al. 2013b) has been used to predict coding sequences in the tulip and lily transcriptomes. Subsequently, the UniProt protein database (Consortium 2015) and the Pfam conserved domain database (Finn et al. 2014) were used to predict protein coding genes. In total 147,101 transcripts of tulip were identified, resulting into 89,530 predicted protein coding genes and 144,801 transcripts of lily, giving rise to 101,312 predicted genes. Those predicted genes represent nearly $50 \%$ of the transcripts in the non-filtered transcriptomes. In a follow-up step, the predicted proteins of tulip and lily were grouped in so-called Orthology clusters using OrthoFinder (Emms and Kelly 2015). The clusters also contained the monocots rice, maize, Brachypodium, sorghum, switchgrass, barley and garlic; and the dicots soybean, Arabidopsis, grape, poplar and tomato. A total of 15,296 orthology groups were found to contain lily and tulip proteins, 10,014 of these also included one or more Arabidopsis proteins. A search for orthology groups that only contained proteins from the bulbous species tulip, lily, and garlic (Kamenetsky et al. 2015), revealed a set of 281 unique groups that might represent bulbous plant specific genes.

To get a better impression of the quality and completeness of the functional annotated datasets, we compared our transcriptomes and annotation with previously published transcriptomes of tulip and lily (Shahin et al. 2012b). Initially, we performed a BLAST search at the nucleotide level to determine how well we covered the transcripts present in these publicly available datasets. Depending on the cultivar we used for this comparison, we found a BLAST hit for $87-95 \%$ of the published tulip contigs and for $80-85 \%$ of the lily contigs. These numbers reveal that we found evidence for the presence of the majority of potential genes in the published datasets in our transcriptomes. Subsequently, we determined how many potential tulip and lily genes with a putative Arabidopsis ortholog were unique in either our transcriptomes, or the published datasets of Shahin and co-workers (2012). To this purpose, a BLAST screening (blastx, e-value cut-off of 1e-5) on the Arabidopsis proteome was performed for the individual datasets. In this analysis we found 1345 and 95 unique tulip hits, for the transcriptomes described in this study and the published tulip datasets, respectively. For lily these numbers were 647 and 164. So on average almost eight times more additional and unique sequences with a BLAST hit to the Arabidopsis proteome were identified in this study in comparison to the previous study. In Supplemental Table 2, an overview is presented of the unique hits in the individual lily datasets as an example. As expected, a large part of the unique sequences in our transcriptomes in comparison to the published transcriptomes resemble genes that are expressed in tissues other than leaves, which was the only tissue sampled by Shahin and co-workers (2012). In addition, sequences were uniquely identified in this study that are potentially encoding for rare and low expressed genes. Examples are three out of 22 known 
members of the novel seed plant-specific family of small peptides encoding genes, ROT-FOUR LIKE I-22 (RTFL 1-22) (Narita et al. 2004).

\section{Transcriptome coverage assessed by the identification of Transcription Factor families}

In the plant kingdom a large number of transcription factor families can be found and they are involved in several processes, ranging from plant development to abiotic and biotic stress responses (Riechmann et al. 2000; Zhang et al. 2011). Transcription factors orchestrate several networks by controlling when and where certain genes will be expressed (Lee et al. 2006) and, therefore, have been well studied and characterized in plants. However, even though they function as master regulators, transcription factors are often expressed at relatively low abundance (Jones et al. 2015). This low level of expression makes transcription factors suitable markers to further assess the sequencing depth and coverage of our two generated transcriptomes. Therefore, a comparison was made between the 42 known transcription factor families in the model species Arabidopsis and rice, and our generated transcriptomes of tulip and lily. For this purpose, the putative transcription factors of each family were identified based on Pfam domains (Finn et al. 2014). The outcome of this analysis is summarized in Table S2.

A large number of transcription factors were identified in the transcriptome data of both lily and tulip with an expected distribution over families, but some families in both tulip and lily seemed to contain more putative members than expected based on their abundance in model species (Fig. 3). Examples are the homeodomain (HB) family and the MYB related transcription factor family. For the FAR1 family, over-representation is observed in comparison to Arabidopsis but the numbers found in lily and tulip, are almost equal in comparison to rice. This might point to a monocot specific expansion of this specific transcription factor family. In general, the number of transcription factor members in a particular family is rather similar in the two bulbous plant species. However, exceptions can be found for the zinc finger LSD and the Whirly family. The LSD family is over-represented in tulip while the Whirly family is overrepresented in lily, based on our datasets. These examples might point to species-specific family expansions, though additional analyses are needed before firm conclusions can be drawn.

A further in depth analysis was made by focussing on the presence of characteristic transcription factor protein domains and comparing them among plant species. In this respect it is good to realize that several families contain a common protein domain and that due to fragmentation of the obtained transcriptomes it may be difficult to distinguish these transcription factor families into sub-classes. Examples are the M-type and MIKC MADS domain transcription factor family clades, AP2 and RAV, B3 and ARF, and HB-other and HB-PHD (Riechmann et al. 2000). In Fig. 4 an overview is given of the distribution of TF protein domains within each species. As expected, the overall distribution is similar between the model species 


\section{Chapter 3}

and the bulbous plants tulip and lily. One of the largest groups of transcription factors, which covers $\sim 13-15 \%$ of all transcription factors of the 42 families, contains a zinc finger domain. The second largest group is represented by the MYB transcription factors ( 12-15\%), followed by the bHLH domain containing transcription factors ( 7-10\%). A major and remarkable difference is observed between monocotyledonous

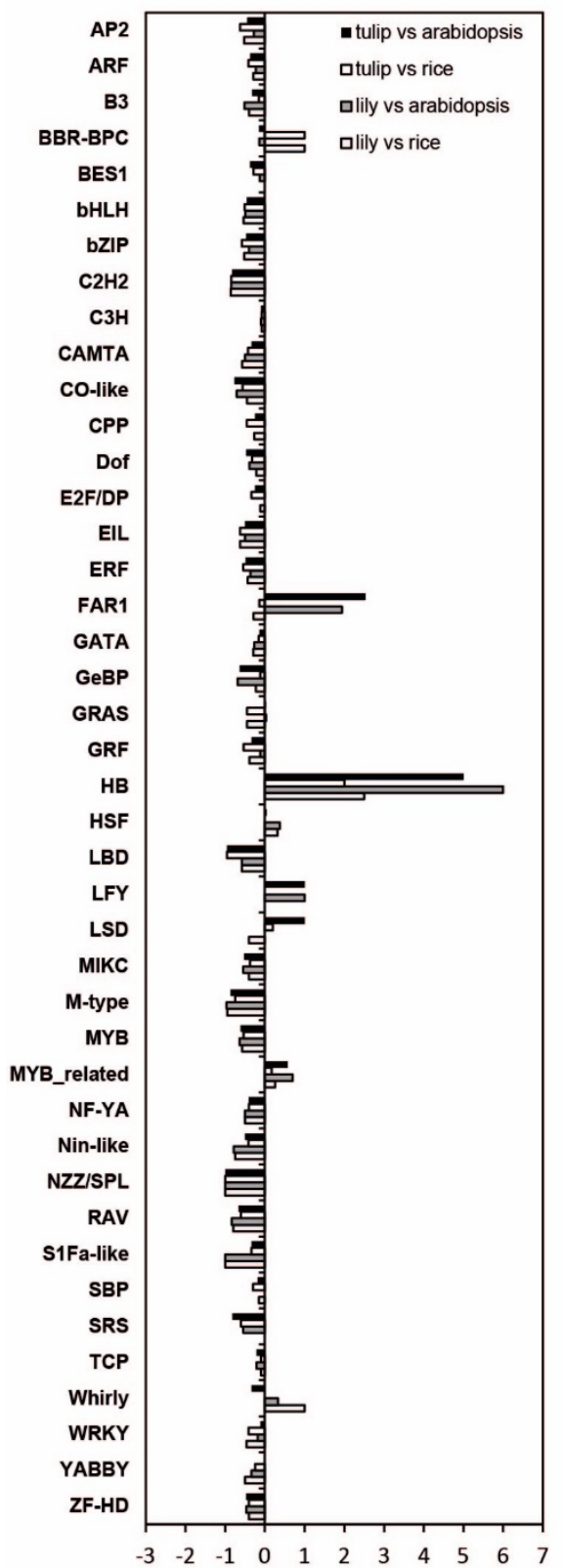

Figure 3. Overview of 42 transcription factor families identified in lily and tulip in comparison to rice and Arabidopsis. The bar represents the relative number of transcription factors present in each family in comparison to the number of transcription factors present in the model species Arabidopsis and rice, respectively. A value below one indicates under-representation in lily or tulip in comparison to rice or Arabidopsis and a value above one shows over-representation. 
and dicotyledonous species for the FARI domain containing transcription factors, as was already mentioned above. Approximately $5-6 \%$ of the total transcription factors used in this analysis has the FAR 1 domain in lily, tulip and rice. Nevertheless, in Arabidopsis only $\sim 1 \%$ of the transcription factors contain this domain. The biological relevance of the expansion of this particular transcription factor family in tulip and lily is currently not known, but it seems not to be an assembly artefact, since the overrepresentation is also found in the completely sequenced rice genome (Sequencing ProjectInternational Rice 2005).
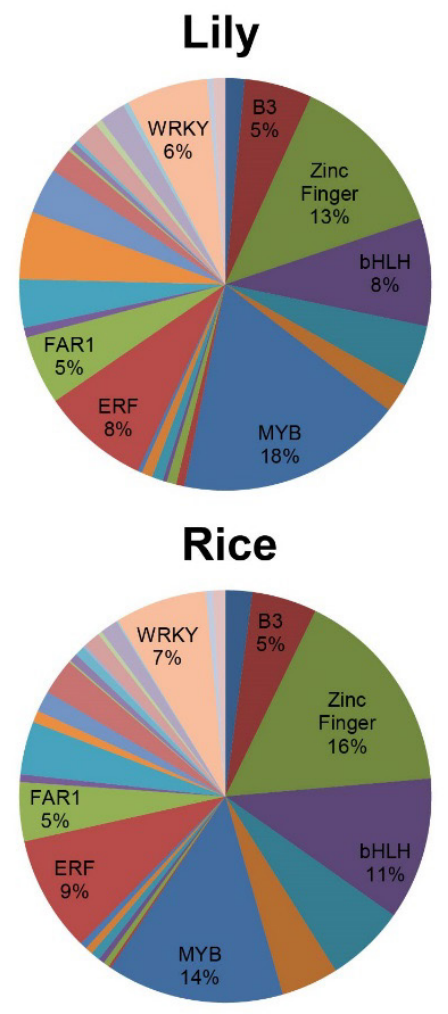

\section{Legend}

$=$ AP2
$=$ bHLH
$=$ MYB
$=$ CAMTA
$=$ EIL
$=$ GeBP
$=$ HSF
$=$ CBFB-NFYA
$=$ S1Fa-like
$=$ TCP
$=$ YABBY

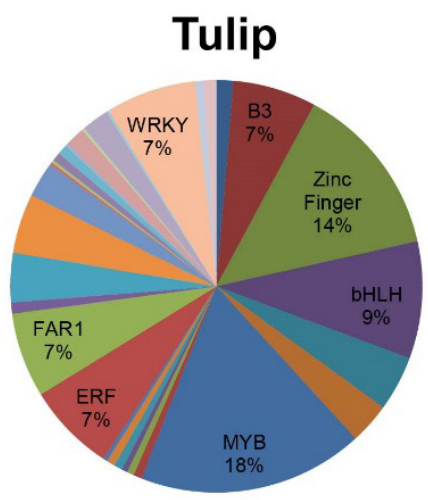

Arabidopsis

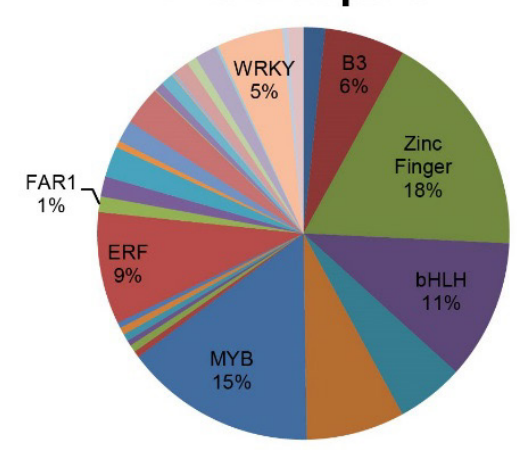

Figure 4. Distribution of transcription factors based on conserved protein domains in lily, tulip, Arabidopsis and rice. The transcription factor family distribution in tulip and lily is similar to the distribution in rice and Arabidopsis. However, in comparison with Arabidopsis, the FAR 1 transcription factor family is larger in the monocots tulip, lily, and rice. 


\section{Mining high throughput data with the Transcriptome Browser: identification of the TCP gene family}

Once a transcriptome is assembled, one of the biggest challenges for researchers is to explore the large dataset in search for sequences with biological relevance. To support in mining data using open sources, we decided to deposit our generated transcriptomes in a web-browser (http://www.bioinformatics.nl/bulbs/db/species/index) based on recently developed open software (Kamei et al. 2016). This web-based interface offers basic bioinformatics search tools, identification of candidate transcripts based on phylogenetic relationships between orthologous sequence data and design of specific and degenerate primers for expression studies of transcripts of interest (Fig. 5).

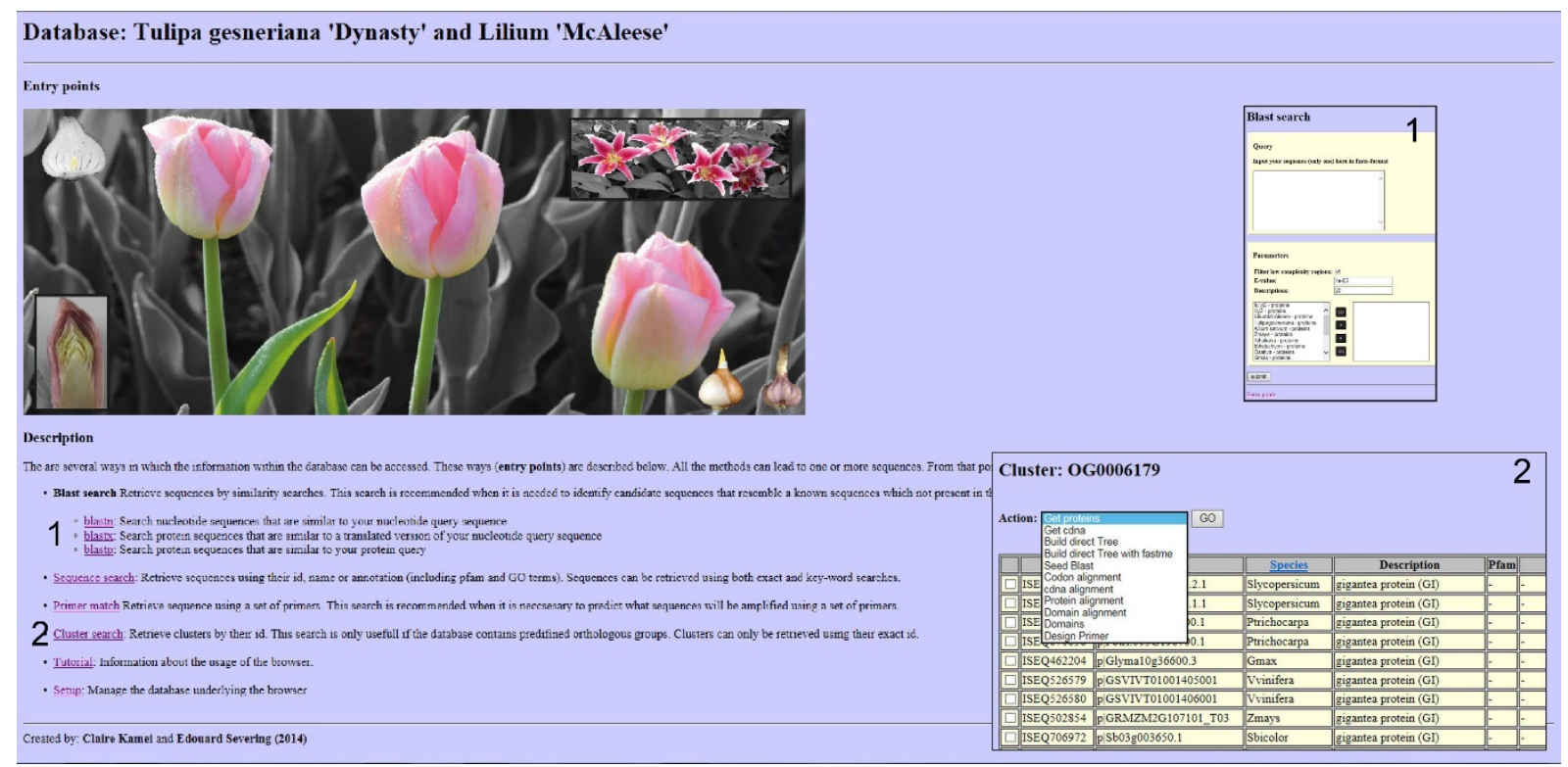

Figure 5. Screenshots of the Transcriptome browser. In panel 1 the interphase of the BLAST search is shown. The input sequence can be blasted against the tulip and lily transcriptomes as well as other plants species such as Arabidopsis thaliana, Oryza sativa and Vitis vinifera. In panel 2 an example is given of the output of the cluster search. Here different actions can be chosen such as protein alignment, primer design and build a direct tree (phylogenetic tree). Note that the browser has a tutorial option, in which the exact procedure how to perform the different tasks and actions is explained.

To explore the usefulness of this data resource, we mined the datasets aiming to identify members of the TCP gene family in lily and tulip. The TCP transcription factor family, named after its founder members TEOSINTE BRANCHEDI, CYCLOIDEA, and PROLIFERATING CELL FACTOR, has in general around 25-30 members in eudicots (Nicolas et al. 2015). TCP genes are expressed in a wide range of tissues and they control flower, leaf, and lateral shoot growth by activating or inhibiting cell proliferation (Martín-Trillo and Cubas 2010) (Nicolas et al. 2015) (Mondragón-Palomino and Trontin 2011). Furthermore, evidence from Arabidopsis expression 
studies indicates that several TCP members are lowly expressed in the above ground tissues (Danisman et al. 2013).

The expected wide-range in tissue and level of expression of TCP genes was our reason to choose this gene family to assess the power of the Transcriptome Browser in mining high throughput sequencing data. All putative lily and tulip TCP sequences were identified by using the sequence search tool (setting Pfam PF03634), followed by seed BLAST analyses with different parameter settings, and an additional manual search scrolling through the orthology (oc) clusters. The Pfam search resulted in 38 tulip and 33 lily transcripts, the seed BLAST search into two additional tulip transcripts and the oc search identified two extra transcripts for each species. This total of 42 tulip and 35 lily transcripts, represented 24 and 22 potential TCP genes respectively.

The following step was to corroborate the TCP identity of the resulting tulip and lily transcripts based on the characteristic features of the TCP domain described by Martín-Trillo and Cubas (Martín-Trillo and Cubas 2010). As shown in Fig. 6, the two putative TCP transcripts identified by seed BLAST search, as well as the remaining lily transcript found by oc search contained only a partial fragment of the TCP domain and this was the reason why they failed to pop-up within the PFAM search. However, they can be considered true TCPs based on their characteristic features. This example shows the power of using the Transcriptome Browser in data mining and highlights the importance of our choice to maintain truncated transcripts into the final assembly.

Although the aim of this study was not to characterize the identity of each TCP transcript found in tulip and lily, we wanted to test the capacity of the Transcriptome Browser in clustering the tulip, lily, rice and Arabidopsis TCP sequences, based on sequence similarity. All lily, tulip, Arabidopsis and rice protein sequences that contained the TCP domain (from the initial Pfam search) were selected to build an unrooted tree using the Neighbour-Joining algorithm (Fig. S1). Once again, the browser was able to distinguish between transcripts from class I and II. Also, most of the clades contained transcripts of all four species, which might help in further approaches to characterize the TCP identity of the tulip and lily transcripts.

Last, we tested the capacity of the "specific primer design tool" offered in the Transcriptome Browser (Kamei et al. 2016). This tool designs primers in unique regions, given a set of similar sequences. PCR amplification of unspecific fragments or fragments without the expected size might indicate assembly errors. Therefore, five TCP genes were selected randomly for each bulbous species. The browser was able to design unique primers in all chosen sequences and PCR amplification with the expected band size was observed in nine out of the ten selected genes (Fig. S2). Overall, this result highlights the power of the de Transcriptome Browser in designing specific and unique primers given from e.g. the members of a gene family. 

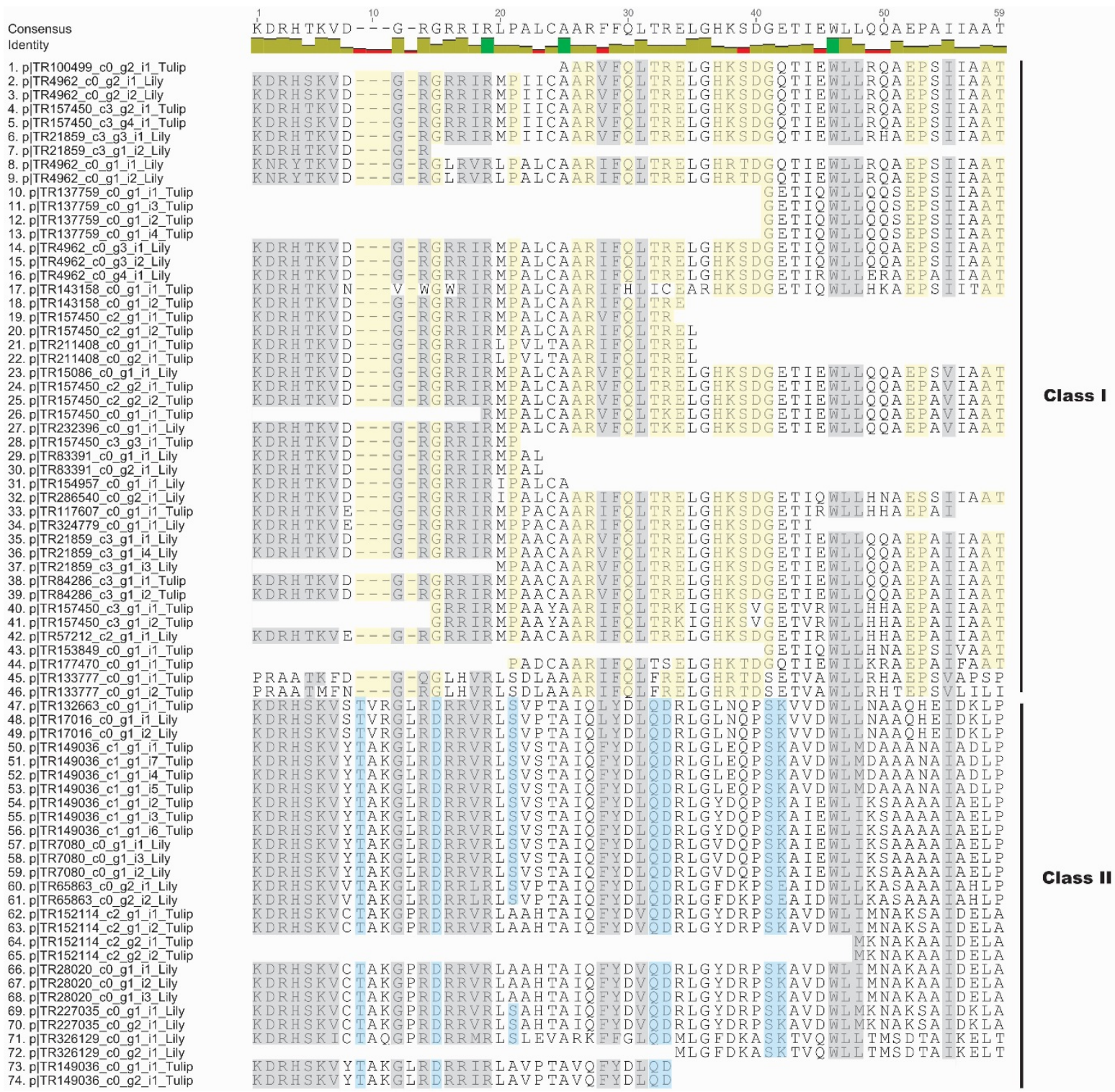

Figure 6. Sequence alignment of the domain of 74 TCP transcripts found in tulip and lily. Sequences are clustered in class I and class II based on the classification by Martín-Trillo and Cubas (MartínTrillo and Cubas 2010). Sequences 64 (P|TR152114_c2_g2_i1_Tulip) and 65 (p|TR152114_c2_g2_i2_Tulip) were found by seed BLAST search only, and sequence 7 (p | TR21859_c3_g1_i2_Lily) was identified by the orthology cluster (oc) search option. Yellow shaded regions indicate characteristic features of class I, blue characteristic features for class II and grey for all other amino acids.

\section{Discussion}

Despite various large-scale sequencing efforts, we still lack a comprehensive transcriptome for many species. In this study a large-scale lily and tulip transcriptome was generated and this resource has been made available in a web-browser for easy mining. 


\section{Filtering out transcripts with low abundance reduced the number of retained plant orthologue hits}

The number of transcripts and predicted genes in our non-filtered transcriptomes may be highly over-estimated taking into account that there are only 27,024 protein coding gene models in the recently sequenced monocot genome of pineapple (Ming et al. 2015), 39,045 genes reported for rice-the monocot model species-(Sequencing ProjectInternational Rice 2005) and 81,791 for tulip, based on a previous transcriptome sequencing effort (Shahin et al. 2012b). Nevertheless, we expect that the large number is certainly not all because of noise, whereas the methodology we selected for sequencing assures high depth coverage and strand specificity. These aspects make the identification of rare and lowly expressed transcripts for both coding and non-coding RNAs possible. Additionally, both tulip and lily are in general vegetative propagated and therefore heterozygosity is maintained, being a source for a higher number of different transcripts.

Although in other bulbous studies filtering out low abundant sequences reduced significantly the number of predicted genes to a level that gets close to what is reported for model species (Villacorta-Martin et al. 2015), we proved that in our data this filtering reduced dramatically the percentage of transcripts with substantial homology to a known plant gene. Therefore, our nonfiltered transcriptomes may not reflect the true number of genes but they rather represent extensive transcriptome coverage for both tulip and lily.

Despite the fact that there is some contamination (non-plant hits) retained in the non-filtered databases, both transcriptomes contained nearly $100 \%$ of a core set of eukaryotic proteins, which is an indication of the completeness of the assemblies. Furthermore, we showed the power of these transcriptomes in finding rarely expressed genes, such as genes belonging to the CLV/ESR family encoding for small size ligands that act as important developmental signalling molecules (Wang and Fiers 2010).

\section{Transcriptome coverage assessment}

In addition to the core eukaryotic proteins, the transcription factor family distribution analysis in tulip and lily has also confirmed the quality of the transcriptome assembly. A large number of transcription factors could be identified even though not all tissues, developmental stages, and common biological process-such as stress responses and floral primordium formation-of the bulbs were collected for RNA-sequencing. To mention an example, tulip tissues were collected from January until May, leaving out the months June to December. During this latter period of time, the floral primordium inside the tulip bulbs is formed (Khodorova and Boitel-Conti 2013) and therefore transcription factors specifically involved in this process might be absent. When zooming in on the members of each transcription factor family found in tulip and lily, some 
families contain more members in comparison to the model species or vice versa. Although, we cannot rule out miss-assembly as a reason for over-representation in particular transcription factor families, a few nice examples of expanded families have been found that based on comparison with other monocots seem to be present and probably unique to monocots or bulbous species. Having more members in a family can be due to the large genome that both tulip and lily have which might be partially due to additional gene duplication events. It will be of interest to study in the future whether there are bulbous-plant-specific functions for these additional genes, proving their biological relevance. Though, before going into laborious indepth functional studies it is essential to confirm a correct assembly of these potential novel genes by wet-lab experiments, other sequencing methods such as PacBio, or using software such as Recognition of Errors in Assemblies using Paired Reads (REAPR) (Hunt et al. 2013).

\section{Functionality of the Transcriptome Browser in mining the extensive tulip and lily transcriptomes}

Mining high throughput data often requires advanced programming skills or access to userfriendly commercial software. Most of the publicly available software tools offer limited options, forcing researchers to use a combination of open software packages, requiring in general different formats and operational systems (Deng 2011). Based on the identification of the putative TCP transcripts for both bulbous species, we confirmed that the Transcriptome Browser (Kamei et al. 2016) represents a reliable and user-friendly web based interface, able to identify gene families and build phylogenetic relationships with other species.

\section{Conclusion}

The methodology implemented in this study to assemble de novo transcriptomes demonstrates that there is a trade-off between transcriptome quality and the amount of information retained. Filtering out data that are considered "noise" improves the values of the parameters that are commonly used to assess the quality of a transcriptome. However, such filtering methods may limit the power of data mining by e.g. reducing dramatically the chances of finding rare or lowly expressed genes. This study resulted in extensive transcriptome resources for both tulip and lily that can be easily mined. The limited number of molecular studies performed in these two bulbous species to date, states the need for such a user-friendly resource. Although, genome sequencing has undergone an enormous revolution over the last decade, it will most likely take some time before a high-quality and well-assembled genome sequence of lily and tulip will become available. Until that moment, the transcriptome browser presented here will be of pivotal importance for gene identification in these two bulbous plant species. 


\section{Methods}

\section{Plant material}

Tulip and lily tissues of several developmental stages were collected throughout the year of 2013 in The Netherlands. Adult tulip bulbs of the cultivar "Dynasty" (Tulipa gesneriana) were planted in October 2012 in the field at Wageningen University $\left(51.9667^{\circ} \mathrm{N}, 5.6667^{\circ} \mathrm{E}\right)$. Tulip bulbscales, axillary buds, stem, leaves and floral bud were collected in January when all organs were entirely below ground; in March when the stem and leaves emerged above ground; and in May during blooming at full anthesis of the flowers. Roots and just initiated and dormant flower buds inside the buds during summer have not been sampled.

Tissues of lily cultivar "McAleese" (Lilium, oriental hybrid group) were collected from regenerated bulblets and from fully grown plants. Regenerated bulblets were obtained by incubating detached bulb-scales in moist chambers without exogenous hormonal application at $23^{\circ} \mathrm{C}$ for six weeks, followed by 12 weeks at $4^{\circ} \mathrm{C}$.. Newly regenerated bulblets were dissected under a stereo microscope and collected at the developmental stages So (proximal side of the explant at the start of the culture); $\$ 1$ (proximal side of the explant at one day after culture); S2 (thickened structures of proximal side of the explant); D (dome formation); P (bulb-scale primordium formation); B (bulblet formation) (Marinangeli et al. 2003). Fully formed regenerated bulblets were also collected at six weeks after culture under $23^{\circ} \mathrm{C}$ (bulblets are thought to enter a resting phase at this moment); and at 18 weeks after culture, from which the first six weeks were at $23^{\circ} \mathrm{C}$ followed by 12 weeks at $4^{\circ} \mathrm{C}$ (bulblets are out of the resting phase and ready to sprout into leaflets or a true stem). In addition to the regenerated bulblets, fully grown leaves, closed and open flowers, stem, and stem axils containing axillary buds were collected at the moment of blooming from greenhouse-grown plants (In the Netherlands; Long day $\left(\sim 16\right.$ hrs of light) conditions and $\left.20-25^{\circ} \mathrm{C}\right)$. After collection of both tulip and lily plant material, the tissues were ground in liquid nitrogen and stored in $-80^{\circ} \mathrm{C}$ until use.

\section{RNA isolation}

Total RNA was extracted from tulip bulb-scale tissue with the Tripure protocol (Roche, The Netherlands) according to the manufacturer's manual, with the addition of $2 \%$ Polyvinylpyrrolidone (PVP, w/v) and $2 \% \beta$-mercaptoethanol $(\beta-M E, v / v)$ to the extraction buffer. Isolated RNA was DNase treated with RQ1 (Promega, The Netherlands) followed by a phenol/chloroform (1:1) extraction and ethanol precipitation. RNA from the other tulip tissues was extracted with the Invitrap spin plant RNA mini kit (Invitek, ISOGEN Life Science, The Netherlands) and DNase treated with DNasel (Qiagen, The Netherlands).

Total RNA from all tissues collected from lily plants was isolated following the Tripure protocol (Roche, The Netherlands) with modifications. The modifications consisted of an initial removal 
of starch using an SDS-containing buffer (buffer I, (Li 2005)) followed by phenol/chloroform extraction; and a final RNA purification of the eluted pellet using the Invitrap spin column (Invitrap spin plant RNA mini kit, Invitek, ISOGEN Life Science, The Netherlands). DNA was removed from the samples by DNAse treatment with RQ1 (Promega, The Netherlands) according to the manufacturer's specification.

Quantity and quality of isolated RNA was assessed by agarose gel electrophoresis and NanoDrop spectrophotometer ND1000. Samples with a 260 to 280 ratio ranging from 1.7 to 2.1 were selected and mixed into equal RNA quantities into a separated lily and tulip pool. These two pooled RNA samples were sent to Wageningen UR Greenomics (Wageningen, The Netherlands) for cDNA library preparation and subsequent sequencing.

\section{cDNA library preparation and sequencing}

A cDNA library for each pooled sample was prepared following the TruSeq Stranded Total RNA Sample Preparation kit with Ribo-Zero Plant (Illumina, The Netherlands). The Ribo-Zero Plant kit removes ribosomal RNA (rRNA) from total RNA using biotinylated probes and the obtained rRNA-depleted RNA is first and second CDNA transcribed keeping strand specificity. Quality and quantity of each library was checked using a Bioanalyzer 2100 DNA1000 chip (Agilent technologies) and Qubit quantitation platform using Quant-iT PicoGreen (Invitrogen, Life Technologies). Library sequencing was done on a HiSeq2000 platform. The tulip and lily transcriptomes raw data were submitted to The National Centre for Biotechnology Information (NCBI) under the numbers SRR3105600 (tulip) and SRR3105700 (lily).

\section{Sequencing analysis}

Paired-end reads were sequenced using Illumina Hiseq 2000. The quality of the reads was examined by FastQC (http://www.bioinformatics.babraham.ac.uk/projects/fastac/). Adapters were removed and paired-end reads were trimmed using Trimmomatic (Bolger et al. 2014b) with settings: "ILLUMINACLIP:TruSeq3-PE-2.fa:2:30:10 LEADING:20 TRAILING:20 SLIDINGWINDOW:4:20 MINLEN:70 HEADCROP:5".

The transcriptomes were assembled de novo using Trinity version 2.0.6 (Haas et al. 2013b) with default settings, except max_memory $150 \mathrm{G}$ and SS_lib_type RF. Transcriptome statistics were determined using the Trinitystats.pl script, which is part of the Trinity package. Transcripts abundances were quantified using RSEM version 1.2.22 (Li and Dewey 2011) with default settings.

To assess the level of contamination contained in both assemblies, NCBI's non-redundant protein database ( $\mathrm{nr}$ ) was searched using Diamond (Buchfink et al. 2015) with default settings and the results were analysed using MEGAN (Huson et al. 2007). CEGMA analysis (Parra et al. 2007) was used as a rough measure of the completeness and quality of the assemblies. 
Coding sequences on the transcripts were predicted using TransDecoder version 2.0.1 (Haas et al. 2013b) as follows: first the longest open reading frames (ORF) were determined and translated using a cut off of 60 amino acids as the minimal protein length. The resulting protein sequences were used as queries to search the SwissProt section of the UniProt protein database (Consortium 2015) with blastp (E-value cut-off $\left.1^{*} e-5\right)$, and they were also scanned for conserved protein domains from the Pfam (Finn et al. 2014) database using Pfamscan. The Blast hits and Pfam results were used as input for the TransDecoder.Predict tool. Subsequently, the longest peptides per transcript on the $(+)$ strand were selected using a custom Python script.

Translated sequences were clustered with orthologous proteins from the monocots rice, maize, Brachypodium, sorghum, switchgrass, barley and the dicots soybean, Arabidopsis, grape, poplar and tomato using OrthoFinder (Emms and Kelly 2015).

\section{Search transcription factor families}

For the identification of transcription factor families, a PFAM analysis was performed on all the proteins present in the transcriptome from both lily and tulip. The families were divided according to the family assignment rules used in the Plant Transcription Factor Database (http://planttfdb.cbi.pku.edu.cn/help famschema.php). Transcription factor families without a Pfam domain were identified with BLAST by using the known Arabidopsis thaliana transcription factors in a particular family.

\section{Tulip and lily transcriptome mining}

Tulip and lily putative TCP transcripts were retrieved using the Transcriptome Browser in three successive steps. The first screen was achieved making use of the sequence search tool, option Pfam (PF03634). In the second step, new TCP transcripts were identified by selecting all tulip and lily transcripts from the first screen and using the "Seed BLAST" tool without default parameters. In the last step every oc cluster containing tulip, lily, Arabidopsis and rice transcripts with a PF03634 hit were screened manually. The TCP domain sequence of each transcript was retrieved manually from the Transcriptome Browser and aligned using Geneious software (Drummond et al. 2010). All Arabidopsis, rice, lily and tulip transcripts resulting from the Pfam (PF03634) search were clustered using the Neighbour-joining tree option of the Transcriptome Browser. Primer design was achieved using the cDNA alignment tool followed by the "Specific" primer design option. The primers used can be found in Table S3.

\section{Data availability}

The Transcriptome Browser containing the Tulipa and Lilium transcriptomes is publicly available via the website http://www.bioinformatics.nl/bulbs/db/species/index. The sequence data are available in the Transcriptome Shotgun Assembly Sequence Database of NCBI 


\section{Chapter 3}

(http://www.ncbi.nlm.nih.gov/genbank/tsa/) under numbers SRR3105600 (tulip) and SRR3105700 (lily).

\section{Acknowledgements}

We thank Van der Gulik Tulpen B.V. for providing Dynasty tulip bulbs, and Hans van der Heijden and Enthius for providing the lily material. We are grateful to Paul Bijman for technical advice and like to thank TTI-Green Genetics and the Dutch Ministry of Economic Affairs for financial support.

\section{Supplementary material}

Table S1. Blast result for the transcripts filtered out of the RNA-seq dataset using commonly applied filter settings. (http://link.springer.com/article/10.1007\%2Fs 111 103-016-0508-1)

Table S2. Overview of lily transcripts with a blastx hit to the Arabidopsis TAIR database and unique in the transcriptome presented in this study or in a recently published lily transcriptome. (http://link.springer.com/article/10.1007\%2Fs 11 103-016-0508-1)

Table S3. Overview of the identified putative transcription factors in lily and tulip, classified over 42 families. (http://link.springer.com/article/10.1007\%2Fs 11 103-016-0508-1) 


\section{Supplementary figures}

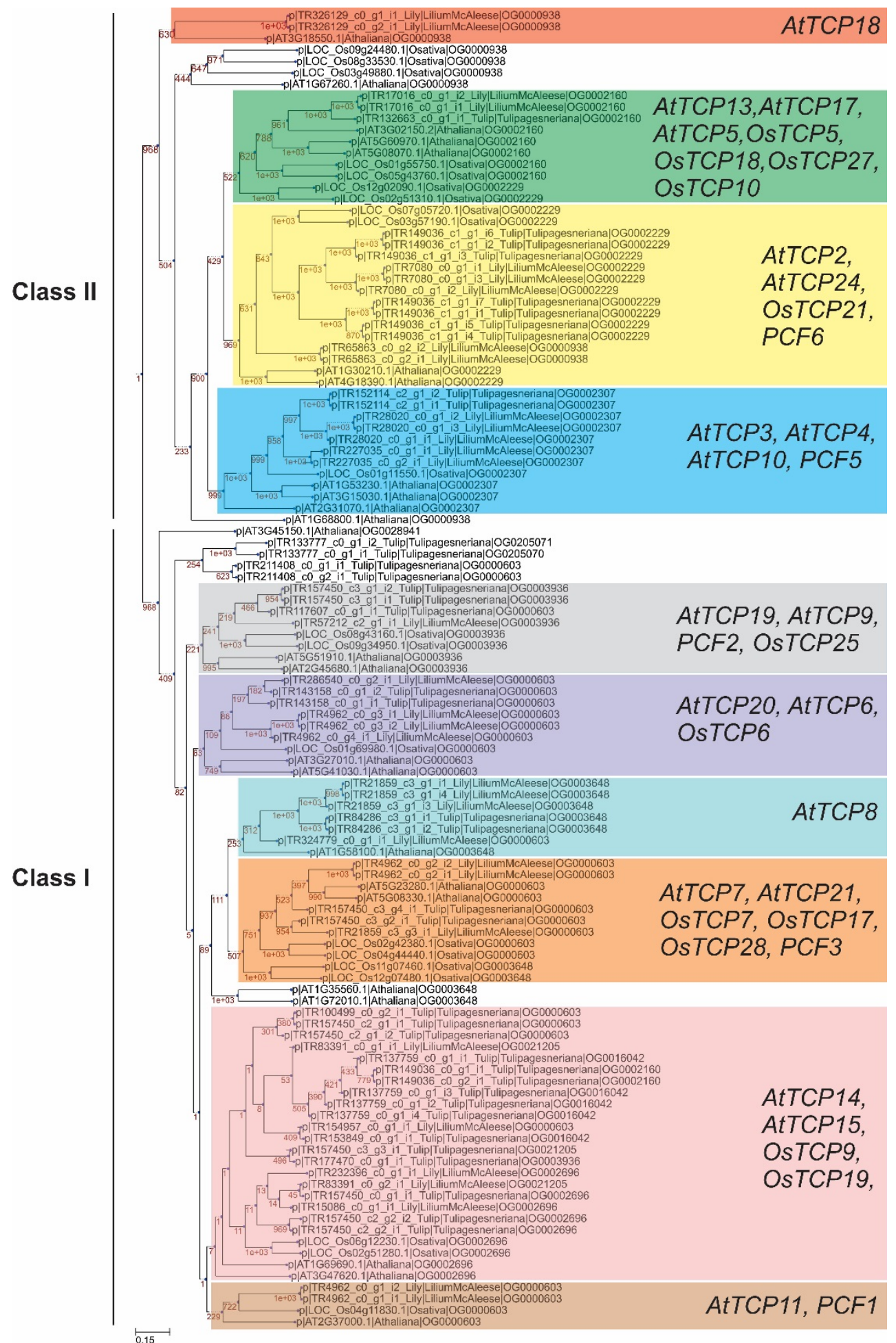

Figure S1. Neighbour-joining tree of the TCP sequences of Arabidopsis, rice, lily and tulip. The sequences from rice and Arabidopsis follow the nomenclature used in the review of Martín-Trillo and Cubas 2010. 


\section{Chapter 3}

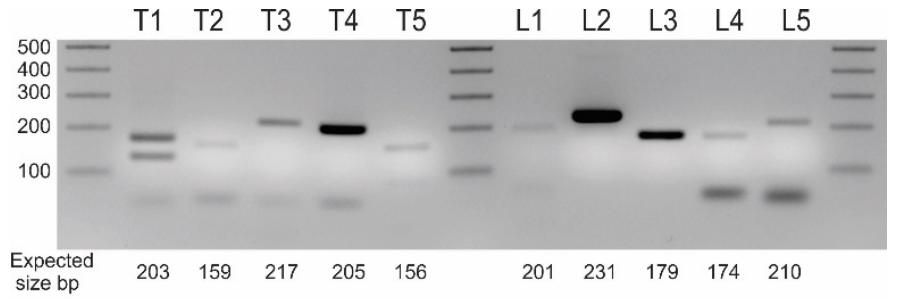

Figure S2. PCR amplification of fragments of 10 putative TCP genes. Five tulip (T1 to T5) and lily (L1 to L5) genes were chosen at random to proof the capacity of the Transcriptome Browser in designing unique primers. PCR amplification was successful in nine out of tten cases. Tl failed to produce the expected fragment size. The primers were designed on the following transcripts: $\mathrm{Tl}=$ p|TR157450_c3_g2_i1_Tulip; T2= P|TR157450_c2_g2_i2_Tulip; T3= p|TR157450_c0_g1_i1_Tulip; T4= p|TR149036_cl_gl_i5_Tulip; T5= p|TR84286_c3_gl_i2_Tulip; L1 = p|TR28020_c0_g1_i1_Lily; L2= P|TR4962_C0_g2_i2_Lily; $\quad L 3=\quad$ p|TR4962_C0_g3_i1_Lily; $\quad L 4=$ p|TR4962_C0_g1_i1_Lily; $\quad L 5=$ p|TR232396_CO_gl_il_Lily. 


\section{CHAPTER 4}

\section{Role of Tulipa gesneriana TEOSINTE BRANCHED 1 (TgTB 1 ) in the control of axillary bud outgrowth in bulbs}

Natalia M. Moreno-Pachon; Marie-Chantal Mutimawurugo; Eveline Heynen; Lidiya Sergeeva; Anne Benders; Ikram Blilou; Henk W. M. Hilhorst; Richard G.H. Immink 


\section{Abstract}

Tulip bulbs reproduce asexually by the outgrowth of their axillary meristems located in the axil of each bulb scale. In every growth cycle the mother bulb is replaced by one or two daughter bulbs, arising from its axillary buds. The number of axillary meristems in one bulb is low -six on average- and not all of them grow out during the yearly growth cycle of the bulb. Since the degree of axillary bud outgrowth in tulip determines the success of their vegetative propagation, this study aimed at understanding the mechanism controlling the differential axillary bud activity. We used a combined physiological and "bottom-up" molecular approach to shed light on this process and found that first two inner located buds do not seem to experience dormancy during the growth cycle, while mid-located buds enter dormancy by the end of the growing season. Dormancy was assessed by weight increase and TgTBlexpression, a conserved TCP transcription factor and well-known master integrator of environmental and endogenous signals influencing axillary meristem outgrowth in plants. We showed that TgTBI expression in tulip bulbs can be modulated by sucrose, cytokinin and strigolactone, just as it has been reported for other species. However, the limited growth of midlocated buds even when their TgTB1 expression is downregulated, point at other factors, probably physical, inhibiting their growth. We conclude that the time of axillary bud initiation, determines the degree of dormancy and the sink strength of the bud. Thus development, apical dominance, sink strength, hormonal cross-talk, expression of TgTB 1 and other possibly physical but unidentified players, all converge to determine the growth capacity of tulip axillary buds.

Key words: Axillary bud, dormancy, apical dominance, TgTB1. 


\section{Introduction}

The outgrowth of axillary meristems determines the branching pattern of a plant as well as the success of vegetative reproduction for several species (Schmitz and Theres 2005). Axillary meristems arise during post-embryonic shoot development at leaf axils. Axillary meristems give rise to axillary buds, which can enter a growth arrest period after forming a few leaf primordia (Schmitz and Theres 2005). In general, after the arrest period, axillary buds sprout into lateral branches which will repeat the formation of the structures developed in the apex of the plant (Bennett and Leyser 2006). In this way, new branches are formed, but also plantlets in numerous vegetative propagated species, including various bulbous plants.

The occurrence of axillary bud outgrowth depends on the developmental stage; physiological condition of the bud (e.g. sink strength); physiological condition of the plant (e.g. apical dominance); and environmental conditions (e.g. temperature, photoperiod, light quality, and nutritional availability) (Bennett and Leyser 2006; Bihmidine et al. 2013; Diaz-Riquelme et al. 2009; Horvath et al. 2003). Axillary bud outgrowth has been studied for long time in relation to apical dominance. Apical dominance is the control of the apex of the plant over the outgrowth of the axillary buds (Cline 1994). Axillary buds repressed by apical dominance are also called para-dormant and they resume growth upon decapitation of the apex (Rinne et al. 2016). Evidence from apical dominance studies indicates that the auxin produced in the apex of the plant (Cline 1996; Cline 2000; Cline et al. 2001; Domagalska and Leyser 2011) and the high sugar demand of the apex (Mason et al. 2014; Barbier et al. 2015) and hence, a kind of starvation of the axillary buds, are the initial inhibitory effectors in axillary bud outgrowth.

Recently, it has been suggested that sucrose is the first trigger to modulate the hormonal networks that control bud outgrowth (Barbier et al. 2015). Auxin seems to interact with the hormone strigolactone to inhibit bud growth (Bennett and Leyser 2006; Brewer et al. 2009; Liang et al. 2010), while cytokinins directly activate outgrowth (Dun et al. 2012; Shimizu-Sato and Mori 2001). Additionally, two other classical and antagonistic acting hormones, gibberellin and abscisic acid, involved in seed dormancy release and dormancy induction maintenance respectively, also seem to play a role in the control of axillary bud development and branching (Elfving et al. 2011; Jiang et al. 2009; Reddy et al. 2013; Yao and Finlayson 2015; GonzálezGrandío et al. 2017).

In the complex network of hormone and sugar signals controlling branching, a TCP transcription factor known as TEOSINTE BRANCHED 1 (TB1) in monocots (Takeda et al. 2003) and BRANCHED 1 (BRC1) in eudicots (Martín-Trillo et al. 2011), has been identified as master integrator (Rameau et al. 2015). A recent study, however, suggested that expression of high BRC 1 levels is not strictly needed for bud outgrowth inhibition in Arabidopsis under all environmental conditions (Seale et al. 2017). Nevertheless, brcl mutants in Arabidopsis present a highly branching phenotype 
due to the constitutive growth of the axillary buds, once the buds are set (Aguilar-Martínez et al. 2007), indicating an important axillary meristem repressing role for BRC1. Furthermore, the expression of $T B 1 / B R C l$ genes is tightly correlated with bud activation potential in a wide range of dicot and monocot species (Manassero Nora et al. 2013). In maize, the TBI locus is thought to contribute to the domestication from its ancestor teosinte (Doebley et al. 1997). TBI overexpression in rice resulted in a reduced branching phenotype, while its mutant exhibited excessive branching (Takeda et al. 2003).

Tulip is a monocot bulbous species in which branching is of high importance because the bulb renews annually through the outgrowth of its axillary buds. Tulip bulbs are equivalent to a compacted plant whose stem shortened into a basal plate that bears modified concentric leaves (bulb-scales); one central apical bud (the Shoot Apical Meristem; SAM) and one axillary bud per bulb scale (Le Nard and de Hertogh 1993; Leeggangers et al. 2013). Note that the outer bulb-scale dries out at the end of the growing season and turns into a so-called "tunica", which will protect the bulb from dehydration during storage time. As a consequence, its axillary bud seems to be located on the outside of the mother bulb. The SAM of bulbs can be found in either vegetative or reproductive state, depending on the size of the bulb (De Hertogh et al. 1983), the environmental conditions, and internal signals. Mature flowering-size tulip bulbs hold on average five bulb-scales and six axillary buds named from inner to outer $A, B, C, D, E$ and $H$, respectively. The outermost bud is always referred to as "H-bud". This is the bud formed from the axillary meristem in the axil of the tunica and its name comes from the Dutch word "huid" which refers to skin (tunica) (De Hertogh et al. 1983).

Tulip bulbs need a prolonged period of winter cold to guarantee successful sprouting of the apical bud and outgrowth of the axillary buds once the temperature rises (Rebers et al. 1994). In that sense, tulip buds resemble tree apical buds. But contrary to most species, axillary bud outgrowth in tulip does not involve shoot elongation but rather "bulbing". During this process, carbon is translocated from source organs into the scales of the axillary buds, and stored into reserve compounds, hence increasing the biomass of the buds. Nevertheless, an exception to this phenomenon occurs in the $\mathrm{H}$-bud, in which sprouting and anthesis take place in spring concurrent with the apical bud (De Hertogh and Le Nard 1993a).

It has been reported that axillary bud activity and growth in tulip bulbs is never completely halted, it diminishes during cold and is promoted in spring (Rees 1968). Once the axillary buds grow out and develop the surrounding tunica, by the end of the summer, they receive the name of daughter bulbs. By then, the energy sources in the scales of the mother bulb have been consumed, the mother bulb senesced, and the daughter bulbs are found in "clumps" attached by the vestigial basal plate of the senesced mother bulb. For the commercial growth of tulips, this moment represents the end of the growing season, and at that moment the "clumps" are lifted from the ground, the daughter bulbs are detached, cleaned, sorted by size, 
and stored at warm conditions $\left(\sim 20^{\circ} \mathrm{C}\right)$ until planting time in autumn (De Hertogh et al. 1983; De Hertogh and Le Nard 1993b; Kamenetsky 2012).

In principle, every mature tulip bulb has the capacity to produce the same number of progeny bulbs as its number of axillary buds, which is on average six (Le Nard and de Hertogh 1993). However, by the end of the yearly growth cycle, only two to three daughter bulbs reach a size similar to the mother bulb and thus, have the capacity to flower in the next growing cycle (Rees 1966; De Hertogh and Le Nard 1993a; Le Nard and de Hertogh 1993). Since tulip bulbs rely on axillary bud outgrowth to reproduce asexually, but this mechanism does not distribute equally the resources to all the buds, it is of great interest to understand the bases of this vegetative propagation mechanism. In this study, we aimed at identifying the factors controlling the differential axillary bud outgrowth in tulip bulbs by combining a physiological and targeted molecular approach.

\section{Results}

\section{Tulip axillary bud growth in the yearly bulb growth cycle}

The natural growth cycle of tulip bulbs was monitored (Figure la-b) with special emphasis on the growth of the axillary buds (Figure 1c-d). The bulbs, of size 9-11 cm in perimeter, had on average five bulb-scales and a tunica, six axillary buds (one axillary bud per bulb-scale) and an apical shoot bearing a floral primordium (hence, also called floral bud) (Figure la). The bulbs were dug out at different time points during the growth season and growth of their axillary buds was measured (Figure 1c).

As stated by other researchers (De Hertogh and Le Nard 1993b; Le Nard and de Hertogh 1993; Rees 1968), we also found that not all axillary buds had the same growth capacity, neither the same growing behaviour (Figure $1 \mathrm{~b}$ ). The outermost axillary bud $(\mathrm{H})$ resembled very much an apical bud in the sense that both experienced shoot elongation, sprouting and bulb renewal (Figure 1a). But contrary to the description of Rees (Rees 1968), which indicated that all axillary buds continue to grow during winter, although at a low rate, we did not detect growth in midlocated (C, D and E) buds during that period (Figure $1 \mathrm{C}$ and Supplementary Figure 1). Once the apical bud of the mother bulb sprouted and its leaves unfolded and turned dark green (Figure 1 a), a boost in growth of A, B and H axillary buds was observed (Figure 1c). Nevertheless, the growth of the mid-located buds resumed mildly, resembling a mild dormant state. By the end of the growing season, all axillary buds made a tunica (moment often referred to as summer dormancy), $\mathrm{A}, \mathrm{B}$, and about half of the $\mathrm{H}$ daughter bulbs formed a reproductive apical meristem (Figure 1d), and their A and B buds (hence, grand-daughter buds) arose later during the storage period. 

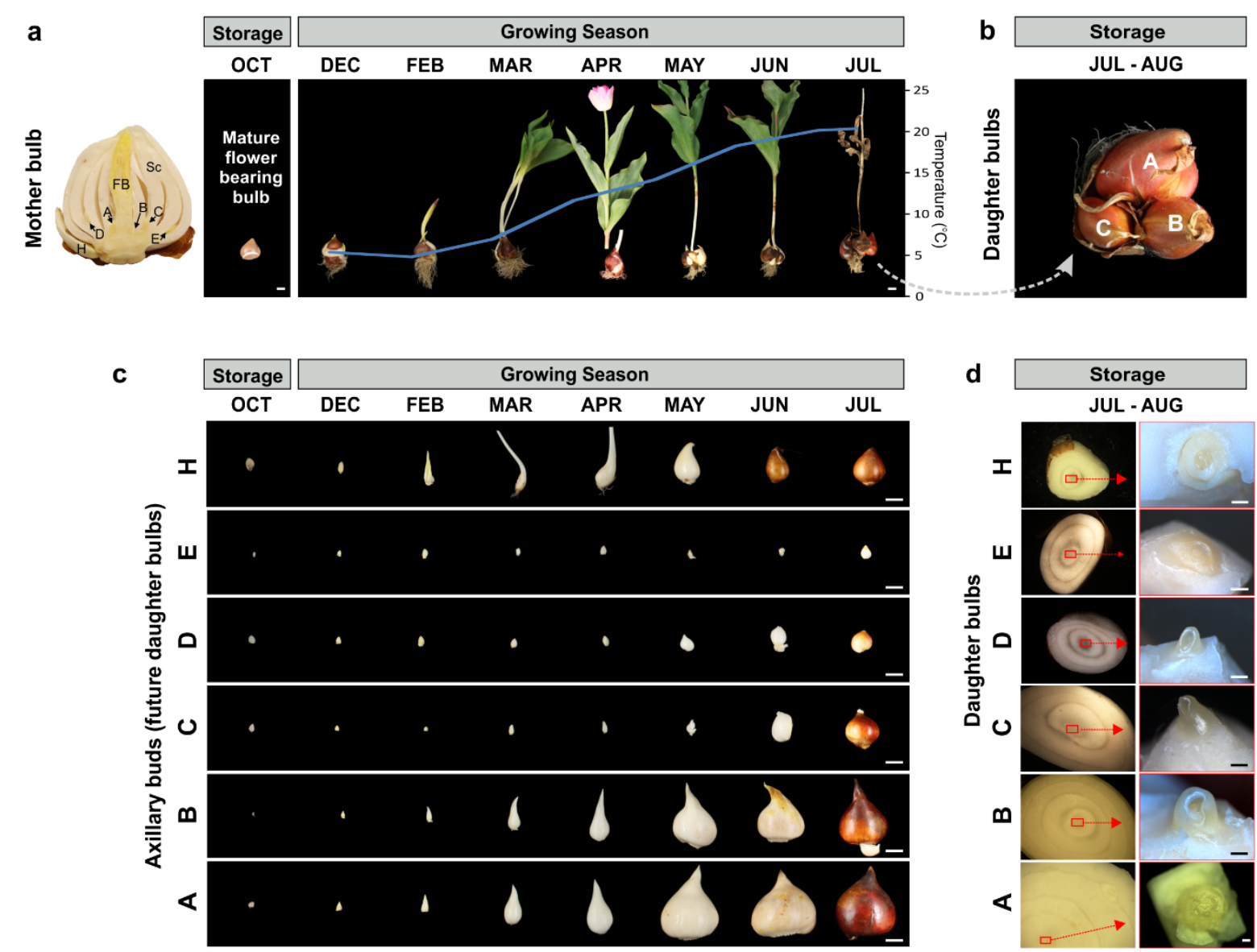

Figure 1. Morphological characterization of axillary bud outgrowth in tulip bulb. (a) Structure of a flowering mother bulb prior to planting (storage period), and its growth development in several time points of the growing season. A, B, C, D, E and H: Axillary buds located and named from inner to outer position in between the bulb scales,.FB: floral bud. Sc: scales. Blue line indicates the average day and night temperature in the ground at $13 \mathrm{~cm}$ depth. Scale bar: $2 \mathrm{~cm}$. (b) Clump of daughter bulbs at the end of the growing season (lifting time). A, B and C: daughter bulbs resulting from the outgrowth of the "A", "B" and "C" axillary buds. (C) Outgrowth of A, B, C, D, E and H axillary buds which were dissected from the mother bulb at different time points of the growing season. $E$ buds dried out in $60 \%$ of the analysed bulbs. Scale bar: $2 \mathrm{~cm}$. (d) Cross section of each daughter bulb at lifting time. The number of scales can be distinguished in the pictures, note that $A$ and $B$ daughter bulbs made more scales than the rest of the bulbs. Red square indicate the position of the apical meristem. Right pictures dissected apical meristem of each bulb, note that $A$ and $B$ buds transitioned into a floral apical bud; $\mathrm{H}$ had a floral bud in $50 \%$ of the analysed buds; and $\mathrm{C}, \mathrm{D}$, and $\mathrm{E}$ buds remained as vegetative buds. Scale bar: $1.5 \mathrm{~mm}$.

The halt in growth of the mid-located buds (C, D, and E buds in this study) during winter suggested a differential control of axillary bud outgrowth in tulip buds, which might be determined by different levels of bud dormancy. The term dormancy as a synonym to temporary growth arrest has been re-defined several times through the years mainly because one definition does not apply to all species. The concept of dormancy in geophytes is still controversial since growth of e.g. the apical bud of tulip bulbs is not arrested after its initiation (Okubo 2012). For practical reasons, we refer in this text to dormancy as the lack of sustained 
growth. The TBI/BRCl TCP transcription factor gene has been used in several dicot and monocot species as a marker to assess bud dormancy (Nicolas and Cubas 2016). To have an extra parameter to assess dormancy in tulip bulbs, we then isolated the putative TBI/BRCl transcript in Tulipa gesneriana and tested its role as a bud dormancy marker.

\section{Identification and gene expression of a tulip TB 1-like TCP transcription factor}

We had previously identified several TCP domain-containing transcripts of tulip and lily, among which, one lily (Lilium oriental) transcript revealed a close sequence homology with Arabidopsis BRC1 (TCP18) (Moreno-Pachon et al. 2016). Based on the lily TB1 (LOTB1) sequence, the tulip orthologue TgTB 1 transcript was isolated. It has been reported that TB 1/BRC 1 proteins share two specific amino acids in the basic region of the domain, which distinguishes them from the other class II TCP transcriptions factors (Martín-Trillo and Cubas 2010). Thus, we aligned tulip TgTB1, lily LOTB1, Arabidopsis AtBRC1 and rice OsTB1 domain sequences and found a high degree of sequence similarity as well as the two shared TBI/BRC1 amino acid features at the same position (Figure 2a).
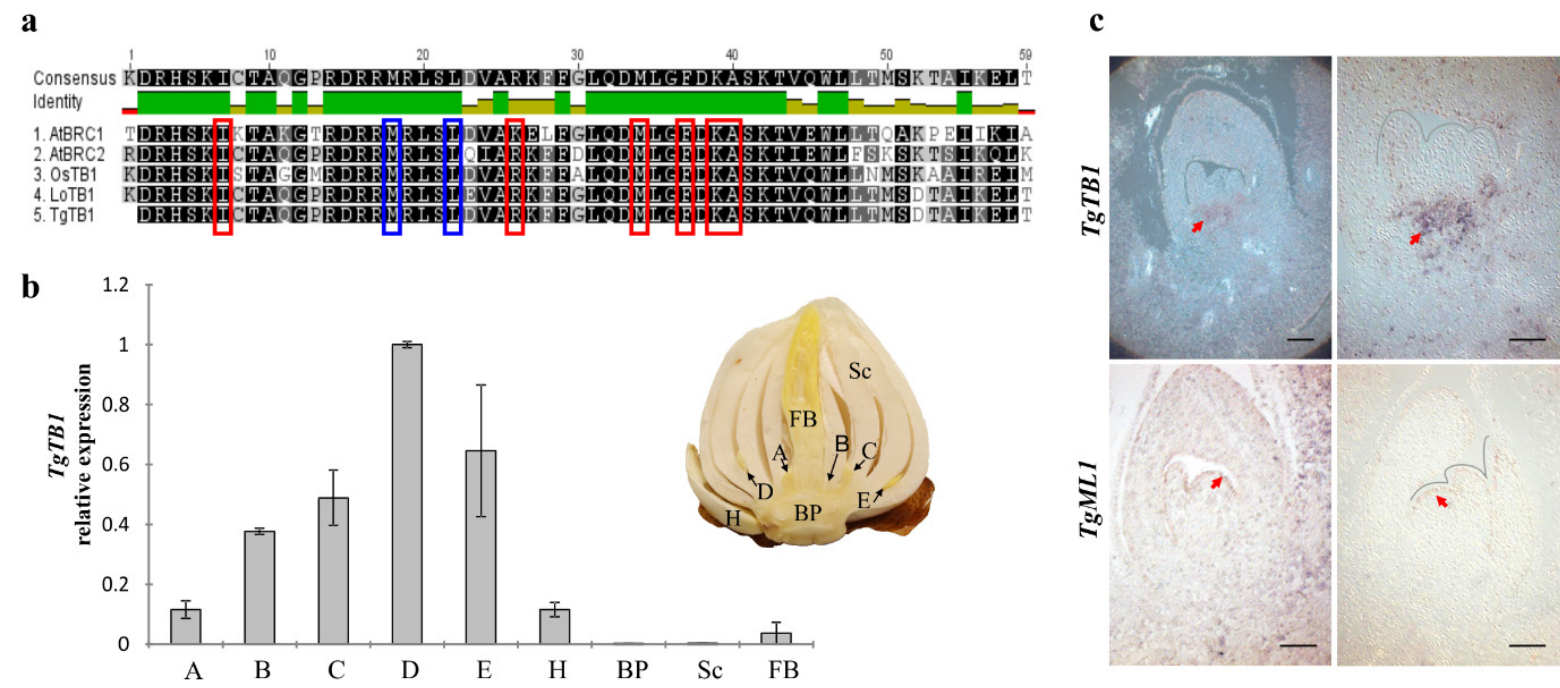

Figure 2. Identification of TgTBI sequence and its expression in different tulip bulb tissues. (a) Protein domain alignment of rice OSTB1, Arabidopsis A†BRC1 and A†BRC2, and putative lily (LOTB1) and tulip (TgTB1) TB1/BRC1 sequences. Red boxes: conserved amino acids in CYC/TB1 proteins. Blue boxes: conserved amino acids in TB1/BRC1/BRC2-like proteins described by (Martín-Trillo and Cubas 2010). (b) Quantitative expression of TgTB1 in different tissues of a bulb at the end of the storage period and one day prior to planting. The expression is relative to the value of $D$ buds. $A, B, C, D, E, H$ : axillary buds located from inner to outer position in the bulb. BP: Basal Plate. Sc: Scales. FB: Floral bud. Data shows the average value of three biological replicates \pm s.e. (c) TgTB 1 in situ hybridization in D buds from bulbs collected at the end of the storage period, one day prior to planting. Longitudinal sections probed with antisense TgTBI and antisense TgMLl as a positive control. The expression of TgTBI was observed at the base of the bud, as indicated by the arrowhead. The expression of TgMLI was observed in the L1 layer of the meristem and scale primordia, as indicated by the arrowhead. Scale bar: $200 \mu \mathrm{m}$. 
$T B 1 / B R C 1$ transcripts have been detected in the meristem and leaf primordia of axillary buds of maize (Hubbard et al. 2002) and in pro-vascular tissues of buds bearing flowers in Arabidopsis (Aguilar-Martínez et al. 2007). To corroborate the true TBI/BRC 1 nature of our identified TgTB1 transcript, the expression of TgTBI was quantified in different organs of the bulb (axillary buds, floral bud, scales and basal plate) at the end of the storage period (prior to planting). Among all tissues tested, TgTB 1 expression was only significantly detected in the axillary buds. Moreover, active $\mathrm{A}$ and $\mathrm{H}$ buds had the lowest expression, while highest expression was found in the dormant $D$ buds (Figure 2b). Subsequently, in situ hybridization was carried out on D buds of bulbs at the end of the storage period and TgTB1 transcripts were detected in the pro-vascular tissue just below the central meristematic region (Figure 2c).

To investigate further the role of TgTB1 as a marker for dormancy in axillary buds, we assessed the expression of TgTBI in daughter bulbs having a so-called "springpartij" phenotype. 'Springpartij' refers to a phenomenon experienced in some tulip bulbs where there seems to be no control in outgrowth of axillary meristems contained in the mother bulb, including the

a

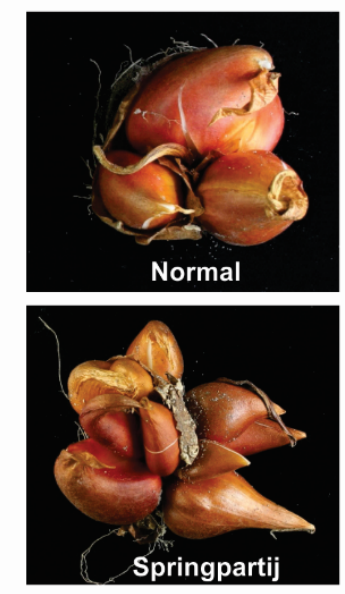

b

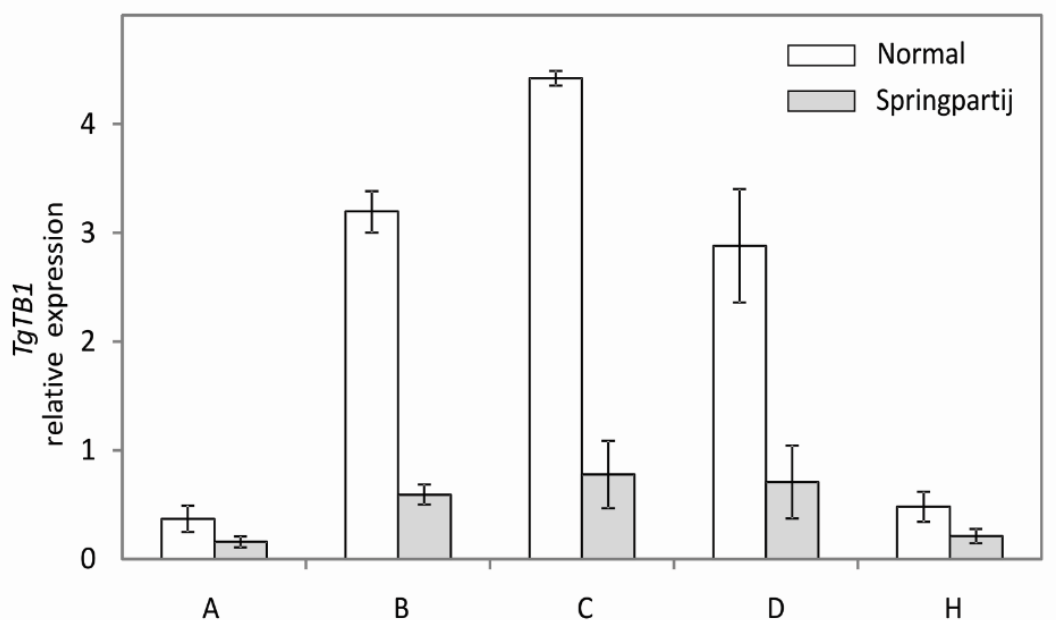

Figure 3. The "Springpartij" phenotype and TgTB1 expression in axillary buds of "Springpartij" daughter bulbs. A. Phenotype of a "Springpartij" clump of tulip daughter bulbs at harvest (down) in comparison to a "normal tulip bulb clump at the same moment (up). Note that all daughter bulbs from the "Springpartij" grew out till more or less similar size. B. Expression analysis of TgTB1. Normal bulbs were used as control. Clumps of daughter bulbs were lifted from the ground and stored at $20^{\circ} \mathrm{C}$ for 10 weeks. The axillary buds of every daughter bulb were dissected and pooled based on their position in the bulb, as a pool of A, B, C, D and $\mathrm{H}$ buds. Data shows the average value of three biological replicates \pm s.e.

axillary buds of the axillary buds (hence, grand-daughter buds). As a consequence, many daughter bulbs of more or less the same size are formed from a single mother bulb at the end of the growing season (Fig. 3A). In general, this spontaneously occurring phenomenon cannot be reverted and in the next growing season the phenotype will be repeated in each of the daughter bulbs. In line with the proposed function of TgTB1 in repressing axillary meristem outgrowth, we found overall low expression of TgTB1 in 'Springpartij' buds (Figure 3b) and did 
not observe significant differences in TgTB 1 expression among the axillary buds, as it was found in normal buds (Figures $2 \mathrm{~b}$ and $3 \mathrm{~b}$ ).

To study the dynamics of TgTBI expression in tulip axillary buds and its correlation with outgrowth, five physiological states of the growth cycle (during storage, at planting time, before anthesis, after anthesis and at lifting) were studied in A and D axillary buds. Those buds were chosen because of their contrasting behaviour in growth during the growing season (Figure 1c), and their different TgTB1 expression at storage (Figure 2b).

a
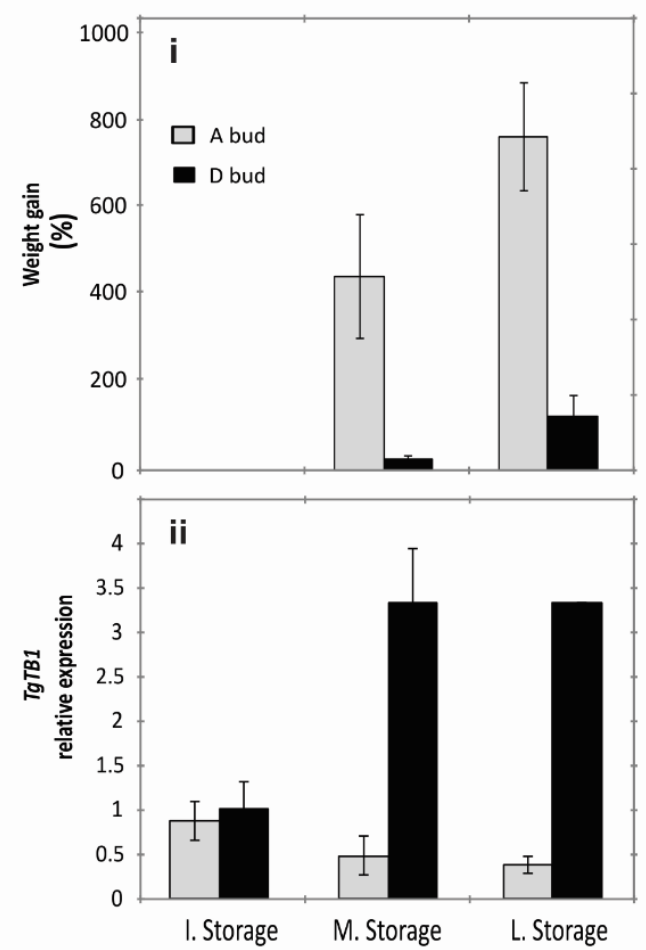

b
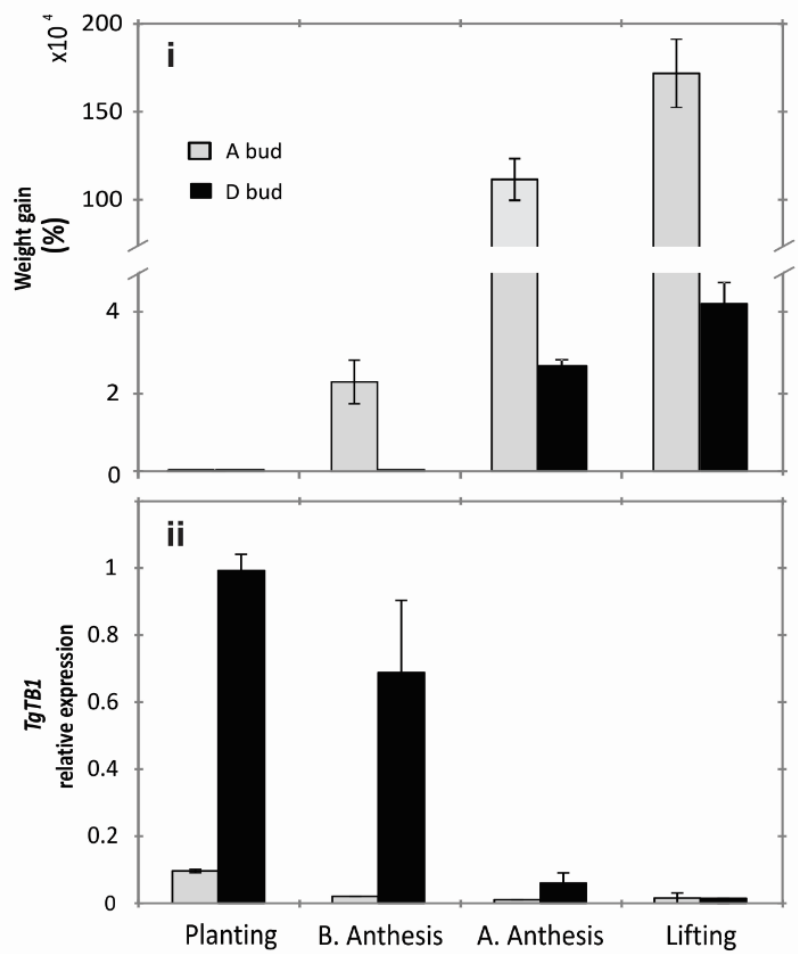

Figure 4. Axillary bud outgrowth in terms of weight increase and TgTBl expression in the innermost "A" and mid-located "D" buds during the tulip growth cycle. (i): Weight increase measured as weight (\%) gained relative to the first time point of each bud. (ii): $T g T B 1$ expression. Data relative to the first time point of D bud. (a) Three time-points were chosen to study TgTBl expression during storage: Initial storage (I. Storage), which was the time after the formation of the A bud; mid storage (M. Storage), corresponding to one month after the formation of the A bud; and final storage ( $F$. Storage), which was two months after the formation of the A bud. (b) Four time-points were studied during the growing season: before planting (late October); before anthesis of the apical bud (March); after anthesis of the apical bud (May); and at the end of the growing season, also called lifting time (July). Data shows the average value of three biological replicates \pm s.e.

The results indicated that during the storage period (time previous to bulb planting), D buds did not significantly grow, while A buds experienced a linear growth increase (Figure 4ai). Regarding the TgTB1 relative expression during this time, there was a dramatic upregulation in $D$ buds by the end of the storage period (late storage time), while TgTBI decreased in A buds (Figure 4aii). Figure 4Bii indicates that after the bulb is planted, TgTB1 relative expression in D 
buds dramatically decreases. In fact, the relative expression of TgTBI in both A and D buds becomes similar after anthesis of the apical bud has taken place (Figure 4bii).

Following the idea of $T B 1$ expression as a marker for bud dormancy, we expected the downregulation of TgTB1 in D buds to result in significant bud growth. From figure 4bi it can be observed that limited, although significant growth was accomplished in D buds in this last period. We discarded the possibility that the limited growth in $D$ buds was due to the lack of vascular connexion with the mother bulb, because both $A$ and $D$ buds showed a main vasculature growing into the basal plate (Supplementary Figure 2).

In summary, TgTBI expression in the selected axillary buds was inversely correlated with their growth pattern during storage $\left(r^{2}=-0.9\right)$ and during the growing season, however to a lower extent $\left(r^{2}=-0.56\right)$. When using TgTB 1 as a marker to assess dormancy, it can be said that $D$ and not A buds enter a dormancy state during lifting time, and this dormancy is only broken in late spring. However, although D buds are freed from a molecular imposed dormancy in spring, their growth is no longer supported.

\section{The role of sucrose in modulating tulip axillary bud outgrowth and TgTB1 expression}

Sugars are stored in the bulb scales as starch and translocated to the sink organs based on their demand (Ho and Rees 1976). Nevertheless, axillary buds obtain most of the carbon from the leaves of the apical bud once it sprouts in early spring (Ho and Rees 1975). Taking into account the reported role of sucrose to break bud dormancy (Barbier et al. 2015; Mason et al. 2014) and the observed correlation of TBI/BRC 1 with axillary bud growth (Aguilar-Martínez et al. 2007; Braun et al. 2012a; Martín-Trillo et al. 201 1; Takeda et al. 2003), we reasoned that midlocated buds might not get enough sucrose to break dormancy in early spring, probably because of competition with the already active buds (e.g. A bud).

To test whether making sucrose equally available to the buds could break dormancy and promote growth in D buds, we cultured them in vitro with or without sucrose in the medium. A buds were used as control. The buds were excised from bulbs stored at $20^{\circ} \mathrm{C}$ for 3 months followed by $4^{\circ} \mathrm{C}$ for other 3 months. These temperature pre-treatments recreated the storage and cold period of their natural growth cycle, resembling the early spring time of tulip growth cycle. As expected, sucrose enhanced the growth in both buds, although to a much lesser extend in D buds (Figure 5). Moreover, TgTBI expression in dormant buds downregulated after the sucrose treatment. Also, sucrose was fundamental for the survival of $D$ buds, which is an indication that the buds are able to uptake the sucrose in the medium. A buds survived the lack of sucrose, but their weight diminished significantly by the end of the experiment. It was intriguing, however that $D$ buds did not grow significantly in the last three weeks of the experiment where sucrose was supplemented. As sugars as an energy source do not seem to 
be limiting, we speculated that other factors, such as hormones might also play a role in controlling the axillary growth capacity of tulip buds.

\section{Hormonal modulators of TgTB 1}

Rameau et al proposed a general model in which $T B 1 / B R C 1$ integrates multiple pathways that control axillary bud outgrowth (Rameau et al. 2015). In this model TBI/BRCl is activated by strigolactone (SL) while repressed by sucrose, cytokinin (CK) and gibberellic acid (GA). However, gibberellins can induce axillary shoot elongation instead of biomass gain in tulip (revised in (Okubo 2012)). To investigate the role of SL and CK in the regulation of axillary bud outgrowth in tulip, we tested the combined effect of sucrose and CK (BAP) in the outgrowth of dormant $D$ buds, since we found that sucrose is required for the survival of this type of buds; while the effect of SL in the active A buds using mannitol as an osmotic control (Henry et al. $2011)$.
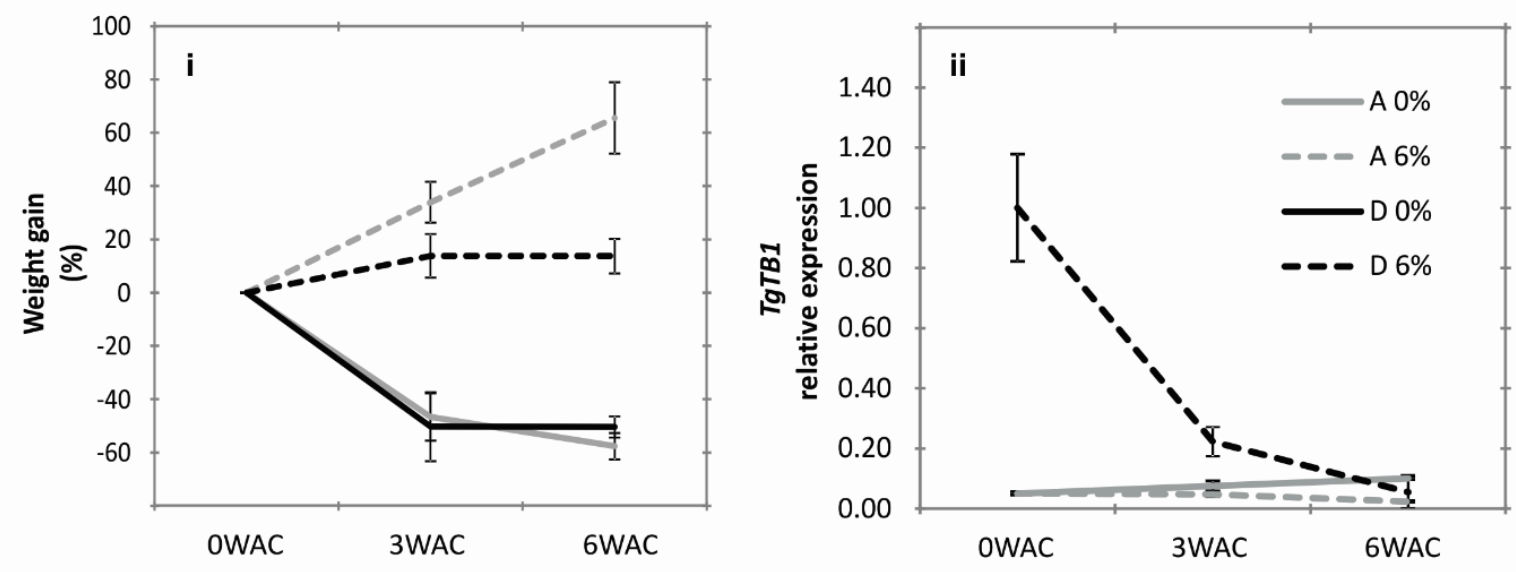

Figure 5. TgTB 1 expression and growth in dormant " $\mathrm{D}$ " and active "A" buds cultured in vitro with $0 \%$ or $6 \%$ sucrose. Bulbs were lifted and stored for 3 months at $20^{\circ} \mathrm{C}$ followed by $4^{\circ} \mathrm{C}$ for 3 months to recreate the end of the cold season from the natural growing cycle. (i): Weight increase measured as dry weight gained (\%). (ii): TgTB I expression, data shown is relative to the first time point of D bud. No TgTB 1 data was available for D buds without sucrose because these buds did not survive. Data shows the average value of three biological replicates \pm s.e.

The results showed that the combination of sucrose + BAP downregulated TgTBI expression faster than sucrose on its own in dormant buds. Nonetheless, after six weeks of culture both, the treatments of sugar and sugar + BAP showed the same capacity to induce bud growth and to downregulate TgTBI to the same level (Figure 6a). With regard to A buds, they experienced a dramatic upregulation of TgTBI expression upon culture with mannitol as well as with the combination mannitol $+S L$, indicating that SL can modulate TgTB 1 expression in tulip bulbs. 


\section{Chapter 4}

Moreover, for both treatments, mannitol and mannitol + GR24, axillary bud outgrowth was halted in comparison to sucrose treatment (Figure 6b).

a
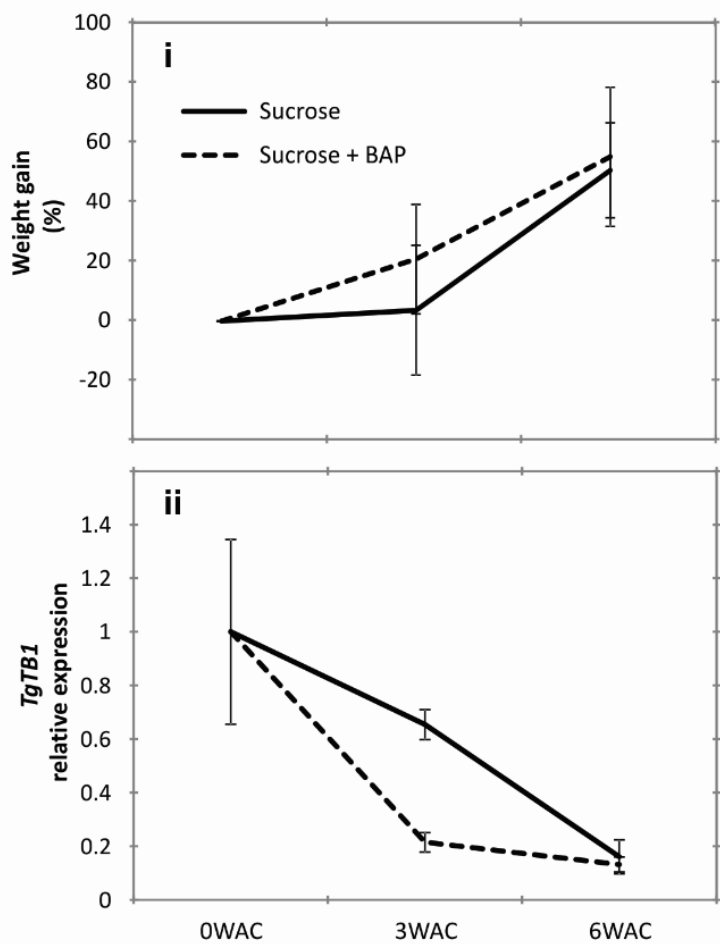

b

Active bud
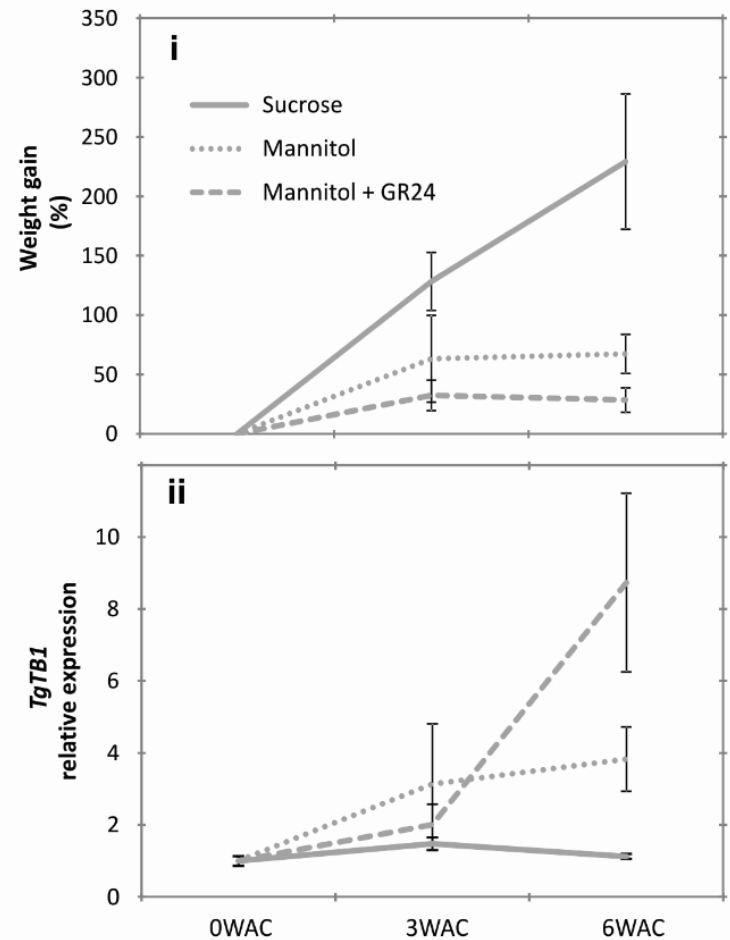

Figure 6. Axillary bud outgrowth and TgTB 1 expression in dormant and active buds cultured in vitro with $6 \%$ sucrose and synthetic hormones. (i): Weight increase measured as dry weight gained (\%). (ii): TgTB1 expression. Data was normalized against the first time point (OWAC). (a) Dormant buds were cultured with sucrose or with the combination sucrose + 25uM BAP (a synthetic cytokinin). (b) Active buds were cultured with sucrose (as a positive control), mannitol (an osmotic control) or mannitol + 0.5 UM GR24 (synthetic strigolactone). Data shows the average value of three biological replicates \pm s.e.

\section{Discussion}

Tulip bulbs can propagate either via sexual or vegetative (axillary bud outgrowth) reproduction and both types of reproduction bring advantages and disadvantages to the survival of the species. Sexual reproduction guarantees the maintenance and broadening of the genetic pool but the transition from seed to a flowering bulb can take up to seven years (Minas 2007; Shahin et al. 2012a). The seedling, which is at the juvenile stage needs to form a small bulb during the first growth cycle and thereafter, the bulb will increase its biomass in every growth cycle until it reaches the reproductive stage (Le Nard and de Hertogh 1993). Axillary bud outgrowth of a flowering-sized reproductive bulb on the other hand, does not broaden the genetic variability but it guaranties the survival of the species by shortening the time needed to produce new flowering bulbs. 
The fact that in tulip not all axillary meristems arrest their growth once they have formed a bud (e.g. A bud), neither their growth results in the same size, might be a strategy of the species to ensure that at least one bud will get sufficient carbon resources to reach a flowering size. This strategy requires a complex mechanism that integrates developmental processes (when the axillary bud is initiated); molecular control of dormancy and dormancy release, sink strength of the buds and likely other or yet unidentified factors.

\section{Axillary bud development and molecular control of dormancy}

When a flowering size mother bulb is planted it has formed all its axillary buds. However, from the early spring to lifting time, grand-daughter buds (axillary bud of the axillary buds) are formed gradually from outer to inner scales (Rees 1968). It has been reported that as in many other plants, once tulip axillary (grand-daughter) buds are formed, they go into dormancy. On the other hand, the A bud escapes dormancy because it is initiated at the time the SAM transitions to the reproductive state, the moment when apical dominance is supposed to be gone (Rees 1968, 1981). We found that A and B buds are actually initiated after the SAM has transitioned to the reproductive state, and that once initiated, those buds never cease to grow. We did not assess the expression of TgTBI at the initiation stage of each axillary bud, thus we cannot prove that H, E, D and C buds go into a dormant state after being formed. However, based on the expression of TgTB 1 in just formed A buds and long-time formed D buds (at initial storage time) it seemed that only then D buds entered a dormant state. The lack of growth of those buds during storage and winter, support the idea that they are dormant.

The term dormancy in flower bulbs is controversial because it has always been assessed in terms of growth or metabolic activity (Okubo 2012). In this study, we assessed dormancy in terms of TgTBI expression and we found that the molecular mechanism of bud outgrowth in tulip is not that different from other plants including model species. As in pea, potato, poplar, crocus and Arabidopsis, TgTB1 can be modulated by sucrose, cytokinin and strigolactone. Nevertheless, TB1 downregulation in dormant buds of tulip does not always result in active growth.

\section{Dormancy and sink strength in tulip axillary buds}

In agreement with Rees, the lack of dormancy in inner buds (A, B) seems to be caused by the lack of apical dominance once those buds are initiated. Moreover, we suggest that this lack of dormancy in inner buds makes them sink organs, even when the bulb is not photosynthetically active. Sucrose is the main product of carbon fixation during photosynthesis (Ayre 2011) and in tulip it is mobilized from the photosynthetic active leaves to the storage organs where it will convert mainly into starch (Rees 1992). Previous studies have used heavy carbon isotope labelled ${ }^{14} \mathrm{CO}_{2}$ to investigate the dynamics of the source-sink relationships of the carbon balance in tulip (Ho and Rees 1975, 1976, 1977). The researchers found that in the absence of photosynthesis (storage and winter period) carbon from the bulb scales of the 


\section{Chapter 4}

mother bulb sustain the slow growth of sink organs, which in order of sink strength are apical bud, root primordia and axillary buds. Later in the growth cycle, in early spring, the leaves become photosynthetic active and both leaves and mother bulb-scales turn into sugar sources for the axillary buds (Ho and Rees 1975, 1976, 1977).

But if sucrose is made available to the axillary buds in early spring, why is dormancy -assessed by TgTB1 expression level- in inner buds (D bud in this study) only broken after flower anthesis, and why is their growth not sustained by then? Our in vitro experiments showed that TgTBI downregulated when the dormant buds were cultured with sucrose. Thus, we suggest that sucrose is not being effectively remobilized to the inner buds before the flower of the mother bulb senesces. In fact, it has been proven that only upon flower senescence, the leaves become the only source and the axillary buds become the strongest sink (Ho and Rees 1975, 1976, 1977). However, the limited growth of D buds, even when TgTB1 expression sharply downregulated both in vivo and in vitro, clearly indicates that TgTB1 is surely not the only regulator of the process.

We propose that a physical factor could be responsible for such limited growth. For example, it has been reported that in perennial trees the release of bud dormancy requires the removal of callose in the plamodesmata in order to restore the symplastic connectivity in the meristem (Rinne et al. 2011; Rinne et al. 2001; Rinne et al. 2016). Although we saw there is a vascular connection between the buds and the mother bulb, it is plausible to think that it might not be very functional when callose is not entirely removed from plasmodesmata in dormant buds, and therefore less carbon resources can be remobilized into those buds. Therefore, investigating the callose deposition and removal in dormant buds might shed more light into the axillary bud outgrowth mechanism in tulip. Alternatively, the number of scales of midlocated buds might determine their growth capacity and sink strength. D buds normally contain only 2-3 scales, while A buds have on average 5 scales. Thus, it is likely that even when the right conditions are met, D buds will never grow as much as A buds because the capacity to store carbon is dependent on the number of bulb scales. In conclusion, the differences in axillary bud outgrowth capacity are regulated by the time of bud initiation in relation with the state of the SAM; the bud dormancy status and the sink strength of the bud. As in other species, TB 1 in tulip is involved in integrating sugar and hormonal signals, but TgTB1 expression on its own does not determine the outgrowth capacity in dormant buds. 


\section{Materials and methods}

\section{Assessing axillary bud growth in natural conditions}

Bulbs of two tulip cultivars -Dynasty and Purple Prince- of size 9-11 (cm in perimeter) were obtained from certified growers in the Netherlands and planted in early November of 2013. Three temperature data loggers (Lascar Electronics) were buried scattered in the field at the same depth as the tulip bulbs. Bulbs were sampled once just before planting and every four weeks during the entire growth season (from planting to lifting). Three additional sampling points were obtained during the storage period for Dynasty bulbs lifted in 2016. For every sampling time-point bulbs were dug out of the ground and dissected in order to collect the axillary buds. The tissue was collected in liquid nitrogen, freeze dried and stored at $-70^{\circ} \mathrm{C}$ until use for RNA isolation. The weight of the axillary buds was also measured at every sampling timepoint. The tissue material and information was obtained in three biological replicates, each consisting of ten bulbs.

\section{Assessing axillary bud growth in vitro}

A and D axillary buds from certified bulbs (cooled at $4^{\circ} \mathrm{C}$ for three months after the storage period, to simulate the cold winter period) were excised from the mother bulb always leaving a piece of the basal plate containing the vasculature of the bud. Excised buds were sterilized by dipping them in $70 \%$ ethanol, followed by $2 \%$ sodium hypochlorite for 20 minutes and three washes with sterile water for one, five and 10 minutes, respectively. The sterile buds were air dried and individually cultured on solid medium ( $1 / 2$ strength MS medium, $0.8 \%$ phytagel $(\mathrm{w} / \mathrm{v})$, pH 5.8 and $0.1 \%$ Dymethyl Sulfoxide (v/v)) supplemented with $6 \%$ or $0 \%$ sucrose $(\mathrm{w} / \mathrm{v}$ ) and/or different concentrations of 6-Benzylaminopurine (BAP) (Sigma) or the synthetic SL (GR24) (provided by Binne Zwanenburg, University of Nijmegen, The Netherlands).

It has been reported that $3 \%$ to $6 \%$ sucrose promotes significant outgrowth of lilium bulblets cultured in vitro (Bonnier and Van Tuyl 1997; Maślanka and Bach 2014), therefore we used 6\% sucrose. For the hormone treatments, three concentrations were tested in order to find the optimal dose for the experiments: $0.5,5$ and $25 \mu \mathrm{M}$ for GR24 and 1, 25, 50 and $100 \mu \mathrm{M}$ for BAP. In vitro cultured buds were grown in a climate room at $24^{\circ} \mathrm{C}$ in dark. The dark environment simulates the natural growth conditions of tulip bulbs underground. For each experiment three biological replicates were done (ten buds per replicate).

The weight of excised axillary buds cultured in vitro was measured at the start and after three and six weeks of culture (WAC). Initial bud fresh weight (iFW) was measured by weighing the pots with medium before and immediately after placing the buds in culture (OWAC). Final fresh weight (fFW) was measured at 3 and 6 WAC and the bud growth was calculated as growth gain in order to correct for the differences in bud size prior to in vitro culture: [FW gain $(\%)=$ 
((fFW-iFW)/iFW) $)^{* 100]}$. After determination of weight, buds were immediately collected in liquid nitrogen, freeze dried and stored at $-70^{\circ} \mathrm{C}$ until RNA isolation.

\section{Identification of tulip TgTB 1}

TB1 sequence was first identified in a lilium cDNA library obtained by Illumina-based RNA sequencing (Moreno-Pachon et al. 2016) and then amplified in tulip using several flanking primers. The detailed method was as follows: all lily transcripts from the library containing the TCP domain were retrieved and compared with Oryza sativa and Arabidopsis thaliana TCP proteins by the neighbour joining clustering method. The conserved features shared by the TB1/BRC1/BRC2-like proteins described by (Martín-Trillo and Cubas 2010) were used to corroborate the true lilium $T B 1 / B R C 1$ gene identity. Several primers flanking the lily TBI domain were amplified in a pool of CDNA from tulip axillary buds in several stages of development. The amplified fragments were sequenced and a region of the TBlgene in tulip was reconstructed.

\section{Quantification of gene expression}

Total RNA was isolated following the hot borate protocol (Wan and Wilkins 1994) with modifications as described by Maia et al. 2011 (Maia et al. 2011): $60 \mathrm{mg}$ of dried ground tissue was homogenized and mixed with $800 \mu \mathrm{L}$ of extraction buffer $(0.2 \mathrm{M} \mathrm{Na}$ borate decahydrate (Borax), 30 mM EGTA, 1\% SDS, 1\% Na deoxycholate (Na-DOC)) containing 1.6 mg DTT and 48 mg PVP40 which had been heated to $80^{\circ} \mathrm{C} .1 \mathrm{mg}$ of proteinase $\mathrm{K}$ was added to this suspension and incubated for $15 \mathrm{~min}$ at $42^{\circ} \mathrm{C}$. After adding $64 \mu \mathrm{l}$ of $2 \mathrm{M} \mathrm{KCL}$ the samples were incubated on ice for $30 \mathrm{~min}$ and subsequently centrifuged for $20 \mathrm{~min}$ at 12,000 g. Ice-cold $8 \mathrm{M} \mathrm{LiCl}$ was added to the supernatant in a final concentration of $2 \mathrm{M}$ and the tubes were incubated overnight on ice. After centrifugation for $20 \mathrm{~min}$ at $12,000 \mathrm{~g}$ at $4^{\circ} \mathrm{C}$, the pellets were washed with $750 \mu$ ice-cold $2 \mathrm{M} \mathrm{LiCl}$. The samples were centrifuged for $10 \mathrm{~min}$ at $10,000 \mathrm{~g}$ at $4^{\circ} \mathrm{C}$ and the pellets were re-suspended in $100 \mu \mathrm{L}$ DEPC treated water. The samples were phenol chloroform extracted, DNAse treated (RQ1 DNase, Promega) and further purified with RNAEasy spin columns (Qiagen) following the manufacturer's instructions. RNA quality and concentration were assessed by agarose gel electrophoresis and Expose ND (Trinean). $500 \mathrm{ng}$ of total RNA was reverse transcribed using the iscript cDNA synthesis kit (Biorad). Quantitative real time PCR (qRT-PCR) was performed using the BioRad CFX96 Real Time PCR System and SYBR Green Supermix (Biorad) according to the manufacturer's instructions. Three biological replicates, each consisting of ten buds, were used as biological replicate. qbase+ software (Biogazelle) was used to determine the expression stability of five tulip candidate reference genes: ELONGATION FACTOR 1 alpha (TgEFla); ACTIN (TgACT); PROTEIN PHOSPHATASE 2 (TgPP2); ADENINE PHOSPHORIBOSYLTRANSFERASE 1 (TgAPTI) and TgTIP4 1 (Supplementary Table 1). Quantification of gene expression was calculated using the $\triangle \triangle \mathrm{C}$ † method, using TgEFla as internal standard. 


\section{In situ hybridization of TgTB 1 and TgML1}

RNA in situ hybridization was performed on cross sections of " $D$ " buds excised from bulbs during storage. The samples were fixed overnight in $4 \%$ formaldehyde in phosphate-buffered saline (PBS), dehydrated in a an ethanol series, embedded in Paraplast Plus (Sigma-Aldrich) and sectioned $12 \mu \mathrm{m}$ thick with a rotary microtome (Zeiss HM340E). the sections were incubated overnight with the sense and antisense probes of each gene fragment. The probes were created with in vitro transcription according to the DIG RNA Labeling Kit instructions (SP6/T7; Roche). The cDNA used for the probe transcription were a 607 base-pairs TgTB 1 fragment using the primers 5'-AGGATCGCCACAGCAAGATA-3' and 5'-AGGCTTTCCCTCGAAGTAGC-3'; and $614 \mathrm{bp}$ TgML1 fragment using the primers 5'-ACAACCGCTGAAAGCAACAT3' and 5'TCATCTGCTGGTCCCCAAAT3'. The sections were observed under light microscopy.

\section{Vasculature staining of axillary buds}

Axillary buds attached to a piece of basal plate were embedded in $4 \%$ agarose and longitudinally sectioned by hand. The sections were stained for lignin with $2 \%$ phloroglucinol $(\mathrm{w} / \mathrm{v})$ in $95 \%$ ethanol. Subsequently, the sections were soaked in $37 \%(\mathrm{v} / \mathrm{v}) \mathrm{HCL}$. Photographs were taken within 30 minutes with a light dissecting microscope.

\section{Supplementary data}
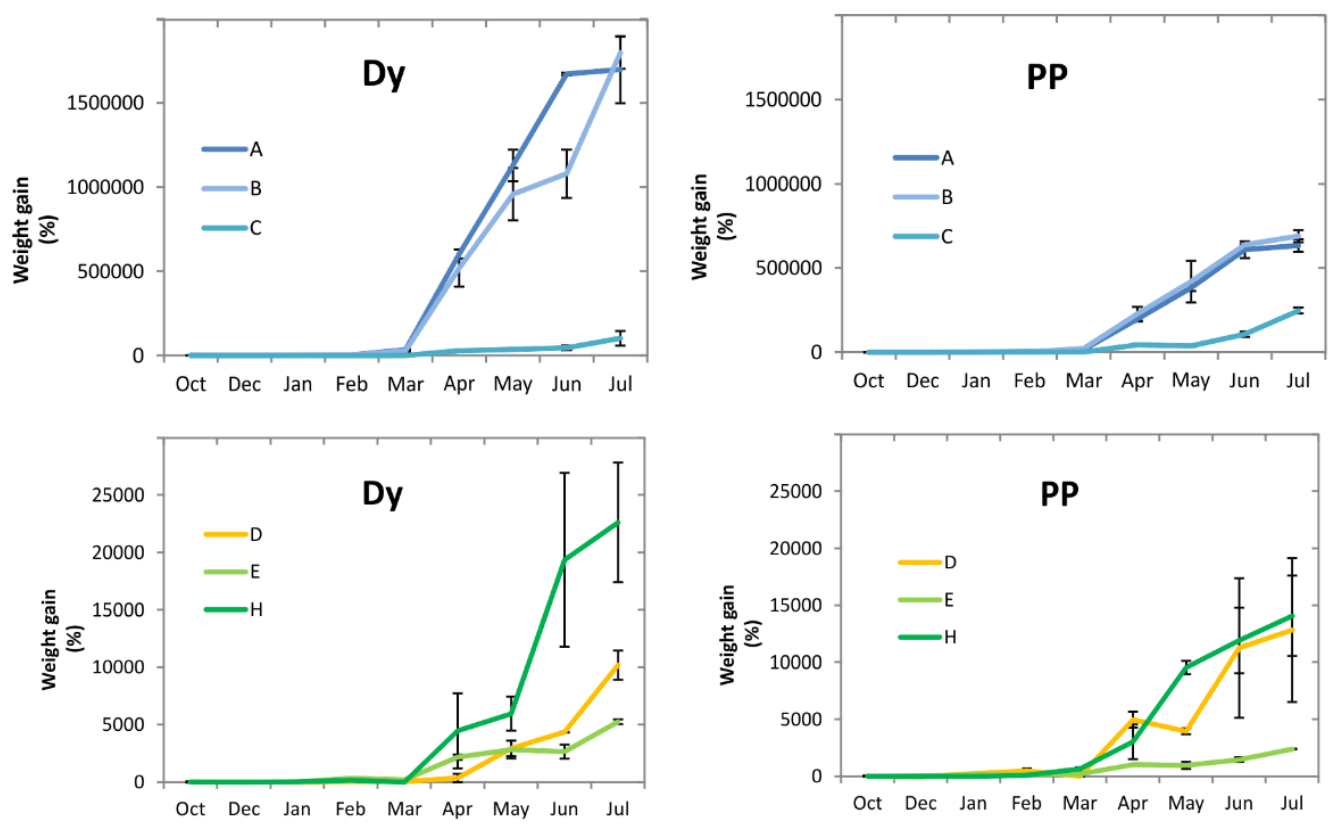

Supplementary Figure 1. Axillary bud growth of two tulip cultivars at several time points of the growing season. Weight increase was assessed as fresh weight gain in percentage (\%). Dy: Dynasty PP: Purple Prince. Note the different $\mathrm{Y}$ axis scale for A-C and D-H buds, respectively. 


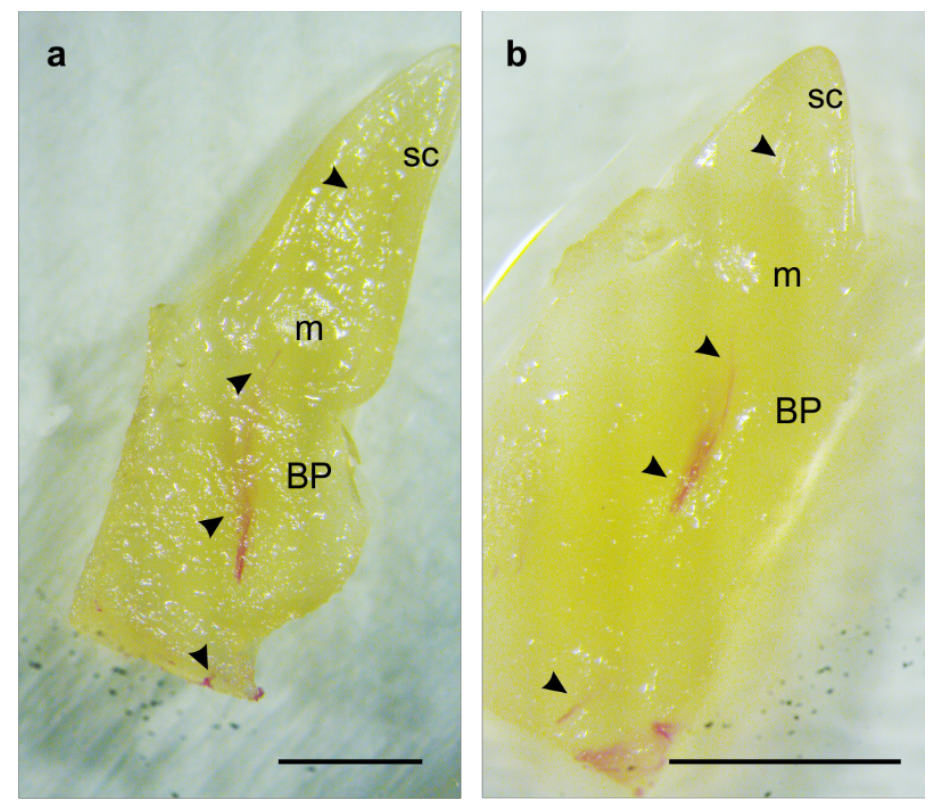

Supplementary Figure 2. Vasculature of "D" and "A" buds. Buds were excised from the mother bulb during the storage period and stained with floroglucinol-HCl which stains lignin. (a) Axillary bud "D". (b) Axillary bud "A". sc: scale, m: meristem, BP: basal plate. Arrow heads indicate the position of the main vasculature, which stained red. Scale bar: $3 \mathrm{~mm}$

Supplementary Table 1. Primers used as reference genes for GPCR analysis.

\begin{tabular}{|l|l|l|}
\hline Gene & TAIR ID & Primer sequence \\
\hline ELONGATION FACTOR la (TgEFla) & Ał5g60390 & $\begin{array}{l}\text { Fw: 5' TGA GAA GGA GGC TGC TGA AA 3' } \\
\text { Rv: 5' TCA CGA TGA CCA GGA GCA TC 3' }\end{array}$ \\
\hline ACTIN (TgACT) & At2g37620 & $\begin{array}{l}\text { Fw: 5' AGC AAC TGG GAT GAC ATG GA 3' } \\
\text { Rv: 5' GGA CAG CCT GAA TTG CAA CA 3' }\end{array}$ \\
\hline $\begin{array}{l}\text { ADENINE } \\
\text { PHOSPHORIBOSYLTRANSFERASE } \\
\text { (TgAPTI) }\end{array}$ & Atlg27450 & $\begin{array}{l}\text { Fw: 5' ATC GCT ACT GGA GGG ACC TT 3' } \\
\text { Rv: 5' AAT TGG AAC TGC GAC AAT CC 3' }\end{array}$ \\
\hline $\begin{array}{l}\text { TgTIP41 } \\
\text { PROTEIN PHOSPHATASE 2A (TgPP2A) }\end{array}$ & At1g13320 & $\begin{array}{l}\text { Fw: 5' TGG CGA GTG GTT TAC TGC TA 3' } \\
\text { Rv: 5' CCG TCT TCA AAT GGT TTG GT 3' }\end{array}$ \\
\hline
\end{tabular}




\section{CHAPTER 5}

\section{Molecular analysis of de novo shoot meristem regeneration in lily bulb scales}

Natalia M. Moreno-Pachon; Harm Nijveen; Annemarieke Borst; Marta CubriaRadio; Wouter Kohlen; Ikram Blilou; Jan Šimura; On đe j Novák; Edouard Severing; Richard G.H. Immink 


\begin{abstract}
Lily has the remarkable capacity to regenerate new plantlets (bulblets) by initiating de novo shoot meristems from excised bulb scales without the addition of exogenous hormones or growth regulators. This capacity makes it a unique system to study de novo regeneration. However, investigating the molecular mechanisms underlying bulblet regeneration in lily is challenging because of the lack of resources and tools, such as a genome sequence and fluorescent marker lines. The aim of this study was to characterize the transcriptional changes occurring from explant excision to the appearance of the pro-meristem. We have performed an extensive and comprehensive transcriptome analysis that provided a first insight into the key molecular processes underlying pro-meristem induction and meristem initiation in lily. We found that wounding activates a very fast regeneration response, probably mediated by APETALA2/ETHYLENE RESPONSIVE FACTORS (AP2/ERF), sUCh as LOERF 115 and WOUND INDUCED DEDIFFERENTIATION 2 (LOWIND2). We propose that these signals trigger polar auxin redistribution, cell proliferation and de-differentiation. Moreover, the timing and level of induction of shoot meristem regulators, sUch as ENHANCER OF SHOOT REGENERATION 2 (LOESR2) and SHOOT MERISTEMLESS (LOSTM) correlated with the regeneration capacity of the scale. Based on the observed conservation of particular key processes and molecular mechanisms in regeneration and meristem establishment, we believe that the obtained results are of value to get a better understanding of plant regeneration capacity in lily and higher plant species in general.
\end{abstract}

Key words: Lily, bulblet regeneration, shoot apical meristem, AP2/ERF transcription factors. 


\section{Introduction}

Plant cell totipotency is the capacity of one cell to differentiate into all the different cell types composing an adult plant. Pluripotency refers to the ability of somatic cells to give rise to most, but not all the cell types of an adult plant (Verdeil et al. 2007). Due to this cell plasticity, plants have the capacity to regenerate new organs and even entire plantlets upon wounding or tissue removal (Iwase et al. 201 la; Liv et al. 2014; Xu et al. 2014). This regeneration process can occur directly via de novo organogenesis or somatic embryogenesis; or indirectly via callus formation (Duclercq et al. 2011; Motte et al. 2014; Ikeuchi et al. 2016).

De novo organogenesis can be achieved ex and in vitro and it starts with re-initiation of cell division, followed by the formation of ectopic meristems. The newly formed meristems will later develop shoots, roots and a vasculature connected to the mother tissue (Ikeuchi et al. 2016). Somatic embryogenesis is the development of a bipolar structure from somatic cells or tissues, from which a new plant can be formed. The bipolar structure contains the future shoot, root, and a vasculature independent from the mother tissue (von Arnold et al. 2002). Indirect regeneration requires the formation of callus before shoots, roots, or embryos arise from the excised tissue. Callus was thought for long time to be a mass of unorganized and dedifferentiated cells (Steeves and Sussex 1972). Nevertheless, contemporary studies indicate that the molecular identity of callus obtained from auxin-rich medium reflects root meristematic identity and its formation resembles the first developmental steps of a lateral or adventitious root (Atta et al. 2009; Sugimoto et al. 2010; Liu et al. 2014).

Shoot regeneration from in vitro cultured explants can be obtained through the manipulation of auxin and cytokinin concentrations in the medium (Skoog and Miller 1957) and has been widely studied in the model species Arabidopsis thaliana using advanced cell biology and molecular techniques (reviewed in (Motte et al. 2014)). As in many other species, Arabidopsis shoot regeneration is preferably achieved using root explants that undergo an intermediate step of callus formation (Cheng et al. 2013). In this two-step method the explants are first cultured in a medium with a high auxin/cytokinin ratio, also called callus induction medium (CIM). The exogenous applied auxins initially accumulate throughout the explant, subsequently generating several local auxin maxima, which will induce cell proliferation from the pericycle cells (Motte et al. 2014). During this callus phase the proliferating tissue expresses the CUPSHAPED COTYLEDON2 (CUC2) gene constitutively (Duclerca et al. 2011). Mutations in CUC genes affect the initiation of shoot the apical meristem (Aida et al. 1999; Takada et al. 2001).

Once formed, the organogenic callus is transferred to a medium with a high cytokinin/auxin ratio, named shoot induction medium (SIM), where shoots will regenerate. Upon SIM culture, WUSCHEL (WUS) expression is induced on the periphery of the callus in slow dividing cells that lack CUC2 expression. A few days later, pro-meristems are formed, in which WUS is upregulated 
in the cells that will turn into the rib-zone of the meristem, and which is surrounded by proliferating CUC2-expressing cells. Subsequently, expression of the auxin transporter PINFORMED $I$ (PINI), and the homeodomain transcription factor ARABIDOPSIS THALIANA MERISTEM LI LAYER (ATML1) is induced in the L1 layer of the pro-meristem. At the same time, expression of another homeodomain transcription factor, SHOOT-MERISTEMLESS (STM), is induced in a ring pattern, in the cells surrounding the pro-meristem cells and its expression gradually moves within this region. Finally, WUS expression is induced in the centre of the meristem and PINI expression becomes polarised towards the periphery, where the leaf primordia will initiate (Gordon et al. 2007).

Shoot regeneration from Arabidopsis root explants is also possible upon direct culture on cytokinin-rich SIM (Atta et al. 2009; Chatfield et al. 2013; Rosspopoff et al. 2017). In this system the pre-existing lateral root primordia (LRP) along the pericycle layer in the root undergo fate re-determination into shoot apical meristems (SAMs) (Atta et al. 2009). Once the root is transferred to SIM, the auxin maxima normally building up in the pre-existing LRP disappear together with the expression of root marker genes, sUch as PLETORA I (PLTI) and SHORT ROOT (SHR) (Rosspopoff et al. 2017). At the same time, the mitotic activity of the LRP is halted. Once cell division resumes, de novo auxin biosynthesis, PINI re-localization and STM and WUS expression take place at the top half of the primordium. As the pro-meristem is formed, the domain of expression of shoot genes resembles the spatial organization of expression in a SAM (Rosspopoff et al. 2017).

In contrast to the above discussed tissue culture approaches, direct shoot regeneration potential without the addition of hormone regulators has been documented in a few species, including lily (Lilium), begonia (Begonia), and Crassula (Walker 1940; Heide 1968; Rost and Paterson 1976). This unique capacity makes these species potential good candidates to study the process of regeneration. Lily bulb-scales have for example, the capacity to regenerate new meristems developing into bulblets when detached from the mother bulb and cultured in just moist and warm conditions. The dynamics of regeneration varies according to the position of the explant within the original bulb scale (apical or basal) (Malik and Bach 2010). Explants from the most proximal (basal) side of the bulb scale have a better competence for bulblet regeneration than the distal (apical) region (Hackett 1969; Long et al. 2004; Yin et al. 2013). Furthermore, variation in regeneration capacity among cultivars has been reported (Kim and De Hertogh 2010). Based on these facts, bulblet regeneration in lily bulb scales constitutes a useful experimental system to investigate and understand the molecular mechanisms underlying de novo regeneration. However, limited research has been done in this species, probably due to its large and complex genome and the lack of biotechnological and molecular tools, such as fluorescently tagged marker gene lines. 
In this study, we aimed to establish a solid basis for a comprehensive understanding of the molecular mechanism underlying de novo shoot apical meristem regeneration in Lilium sp, using an RNAseq-based approach. Detailed analyses of differential gene expression in two cultivars with significant difference in regeneration capacity showed that de novo meristem formation is initiated within a few days after wounding of the scales and culturing of the explants. Already three days after start of the experiment, the relative abundance of cell cycle genes is increased and particular cells within the adaxial scale region start dividing. This process results in oriented and well-defined cell divisions giving rise to the formation of a meristematic dome. Among the group of genes that are initiated already one day after start of the experiment and prior to the first cell divisions, is a lily gene with high sequence similarity to ETHYLENE RESPONSE FACTOR115 (ERF115). ERF115 was recently identified as a key gene providing regenerative potential to Arabidopsis plant cells (Heyman et al. 2016), making it a potential candidate for one of the initial triggers of shoot regeneration in lily.

\section{Results}

\section{Morphological characterization of bulblet regeneration capacity in three lily cultivars var Oriental}

To get insight in the molecular regulatory mechanisms underlying de novo organogenesis in bulblet regeneration, detailed knowledge about the temporal cellular and developmental changes during the regeneration process is essential. Furthermore, it would be beneficial to have material available from lily cultivars with contrasting regeneration capacity, enabling comparative gene expression analyses. With these criteria in mind, bulblet regeneration of the lily Oriental cultivars 'C15', 'McAleese' and 'Paradero' was dissected at the morphological level. Regeneration always occurred at the adaxial side and most proximal end of the explants, as it has been reported previously (van Aartrijk and Blom-Barnhoorn 1983) (Figure 1a).

Four main stages of regeneration could be identified, taking into account a previous description and classification of bulblet regeneration from scales (Marinangeli et al. 2003) (Figure 1b). In a developmental order they were: $S$, representing the time where no signs of regeneration were visible yet; $D$, referring to the appearance of pro-meristems which look like "domes"; $\mathrm{P}$, representing a meristem with the first scale primordium; and B, which we considered as a regenerated bulblet, regardless the number of scales formed. Marinangeli et al. reported a stage between S1 and D, called S2 and characterized by the thickening of the adaxial-proximal end of the scale explant. However, we did not observe this thickening consistently prior to pro-meristem formation in the analysed cultivars. For this reason, we did not include the $\$ 2$ classification. 
Taking into account previously reported differences between apical and basal scale explants (Hackett 1969; Long et al. 2004; Yin et al. 2013), and to obtain a global view of regeneration capacity and timing, we initially assessed the bulblet regeneration capacity of apical and basal scale explants of each cultivar at 10,20 and 35 days after start of the culture (DAC; Figure 1c).

(a)
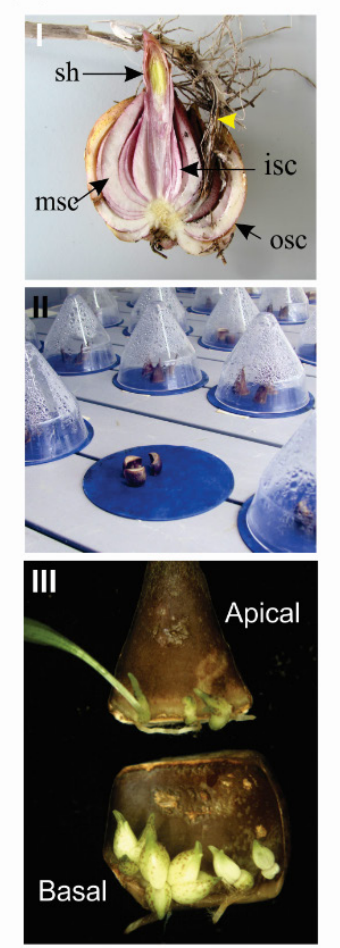

(b)

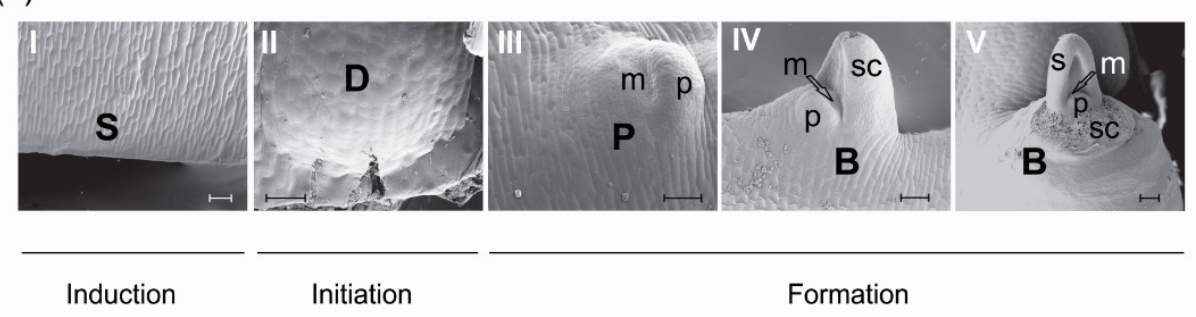

c)

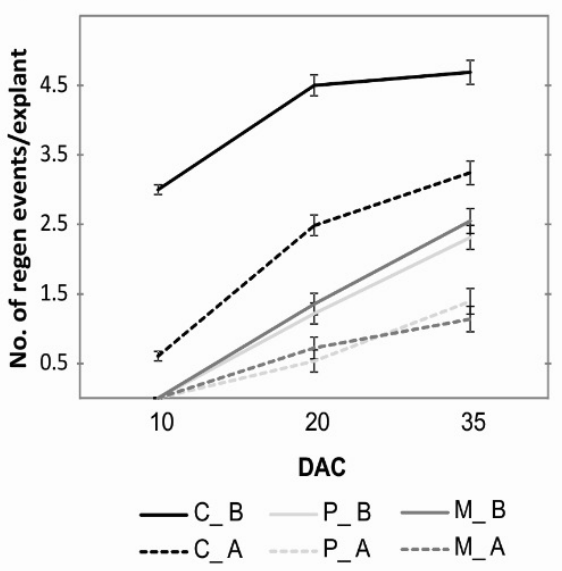

(d)

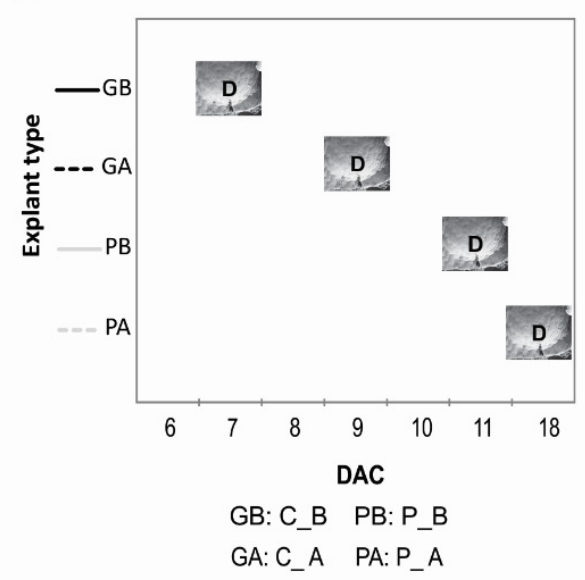

Figure 1. Morphological dissection of bulblet regeneration in three lily cultivars. (a) Lily bulb scales regenerate ectopic shoot meristems (bulblets) upon detachment and culture in warm and moist conditions. I. Cross cut through a lily bulb from which mid-located scales have been isolated for the regeneration experiment. osc: outer-located scales. msc: mid-located scales. isc: inner-located scales. sh: new apical shoot. Yellow arrow head: dried stem from the previous growing season. II. Culturing of lily scale explants on a wet filter paper on a germination table. III. Final regeneration result after 35 days of culture for a representative apical and basal explant. (b) Scanning Electron Microscopy (SEM) analysis of the most basal and adaxial region of scale explants. Different developmental stages of bulblet regeneration are shown and classified according to (Marinangeli et al. 2003). I. S stage, S: refers to "scale" and corresponds to the period where the scale explant recovers from the wound and finishes when the first pro-meristem (dome-structure) appears. II. D stage, D: "dome", corresponds to meristem initiation. III. P stage, P: "primordia", corresponds to the start of bulblet formation, the meristem and first new scale primordia are clearly differentiated. IV. B stage with one bulblet scale, B: "bulblet"; V. B stage with two bulblet scales from which one has been removed. m: meristem; p: primordia; sc: scale. scale bar: $200 \mu \mathrm{m}$. (c) Bulblet regeneration capacity of apical and basal explants of three lily cultivars, assessed as the number and timing of regeneration events at stage D or beyond. C_B: C15 cultivar, Basal explant. C_A: C15-Apical. P_B: Paradero cultivar, Basal explant. P_A: Paradero-Apical. M_B: McAleese cultivar, Basal explant. M_A: McAleese-Apical. DAC: days after the start of culture. Means \pm s.e. of $n=10$ explants (three biological replicates). (d) Timing of pro-meristem appearance in four type of explants differing in regeneration capacity. GA: "Good-Apical" explant, corresponds to C15_Apical. GB: "Good-Basal" explant corresponding to C15_Basal. PB: "Poor-Basal" explant corresponding to Paradero_Basal. PA: "PoorApical" explant corresponding to Paradero_Apical. DAC: days after the start of culture. 
The regeneration capacity was analysed in both terms of timing and number of regeneration events. The first visual regeneration events for cultivar $\mathrm{C} 15$ emerged already within the first 10 days after start of the culture (DAC), while it was later than 10 DAC for Paradero and McAleese. Also, the number of regeneration events per explant at each investigated time point was higher in C15 than in Paradero and McAleese. In general, C15 showed higher regeneration capacity than McAleese and Paradero, while there were no significant differences between McAleese and Paradero ( $p<0.001$; Figure $1 c)$. For all three cultivars tested, basal explants had a better propagation capacity compared to apical ones, which was reflected in both speed of regeneration and number of regeneration events.

Based on this global investigation of the bulblet regeneration process in the three different cultivars (Figure 1C), we designated $\mathrm{C} 15$ as good regeneration system (G), and Paradero and McAleese as poor regeneration systems (P). Because of the similar response of Paradero and McAleese, we performed all further experiments with Paradero as poor regeneration system. Secondly, we distinguished apical and basal explants, because of the consistent higher regeneration capacity of basal scale explants (Figure 1c). Taking this into account, we refer from now on to four types of explants: GA (good regeneration system-apical); GB (goodbasal); PA (poor regeneration system-apical) and PB (poor-basal).

\section{Molecular control of de novo bulblet regeneration}

Next, we performed a detailed analysis of the dynamics of the early stages of regeneration up to the ' $D$ '-stage, since we were interested in the changes prior to pro-meristem formation. We found that the first pro-meristems were visible at 7DAC in GB, at 9DAC in GA, at 11DAC in PB and at 18DAC in PA (Figure 1d). These distinct responses of the different explant types provided a good basis for further in depth analyses at the cellular and molecular levels. Since we were especially interested in the early regeneration signals and regulatory processes, we decided to focus on the first 9 days of explant culture.

The four selected explant types (Figure 1d) showed significant differences in the timing of meristematic dome initiation and regeneration capacity (Figure 1c), and we expect that this must be reflected in the transcriptional changes during the meristem induction phase (S). Hence, RNA sequencing (RNAseq) and quantification was performed on RNA isolated from the most proximal cells of the four types of explants at different time points during the S-phase and up to the D-phase of regeneration. Note that in this time span only GB and GA explants showed meristematic domes at the end (7 and 9 DAC respectively; Figure 1d).

Upon RNAseq, the transcriptome was assembled de novo using Trinity software (Grabherr et al. 201 1a). 183,895 trinity "genes" were obtained and for 60,322 of these transcripts an annotation could be obtained based on a BLAST search against the Arabidopsis and rice transcriptomes. In the rest of this study, we refer to the Lily transcripts with a name based on the Arabidopsis 
annotation, but preceding the initial "LO", standing for Lilium oriental. Principal Component Analysis (PCA) of all expressed transcripts at all time points indicated that the cultivar and the timing of collection (0-1 DAC vs 3-9 DAC) explained $40.3 \%$ and $13 \%$ of the total expression variation, respectively (Figure 2a). Hence, besides the differences in the genetic background, there was a clear overall transcriptional change around 3DAC in the regenerating explants, regardless their origin in the bulb-scale (basal vs apical).

(a)

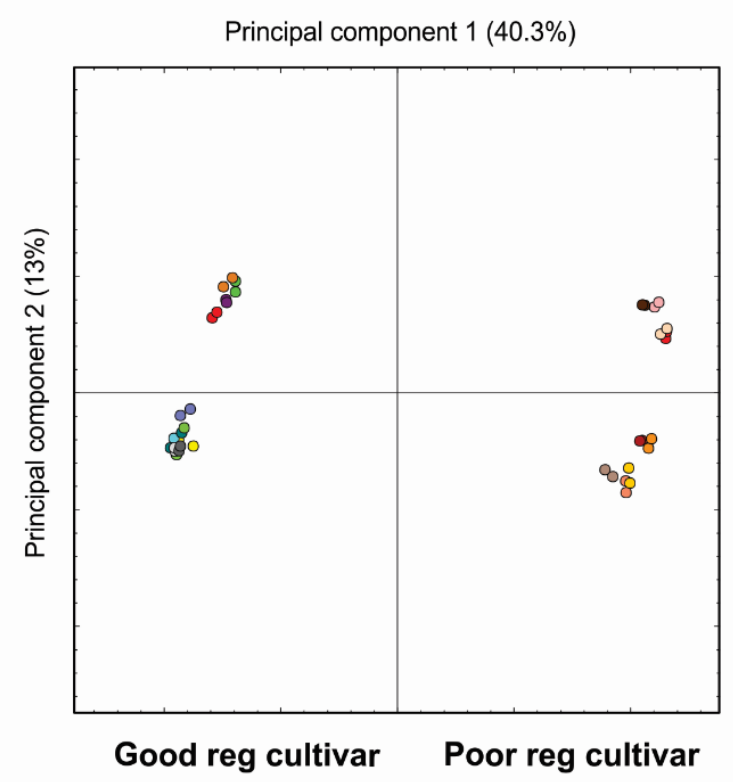

(b)

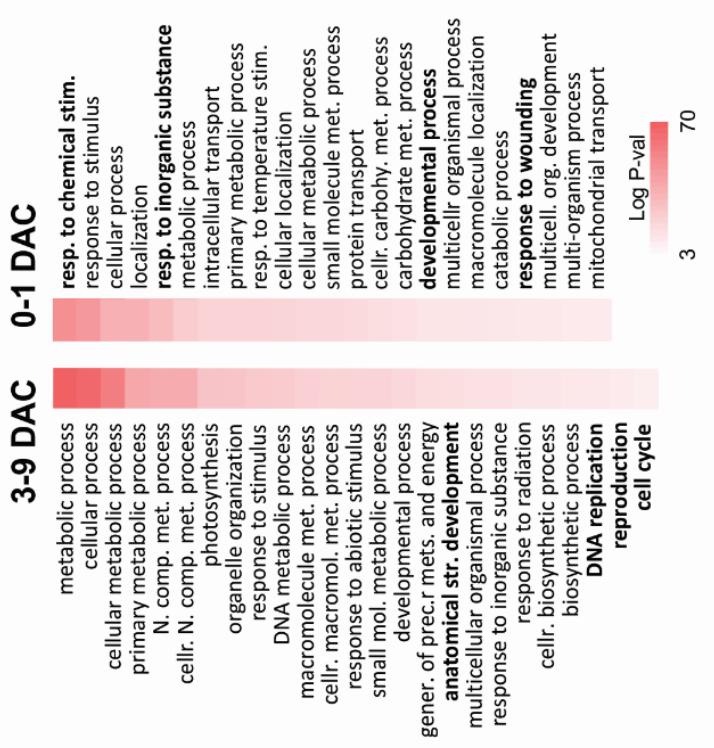

Figure 2. Global analysis of transcriptional dynamics during the first nine days of bulblet regeneration in four explant types. (a) Principal Component Analysis (PCA) of the transcriptomic data generated for apical and basal explants of a good and a poor regenerating cultivar at different time points of bulblet regeneration. Principal component 1 separated the two cultivars, while component 2 separated early (0-1 DAC) from late (3-9 DAC) time points. Dots with same colour represent technical replicates. Good regenerating cultivar: C15. Poor regenerating cultivar: Paradero. (b) Over representation analysis of gene ontology $(G O)$ clusters significantly different between $0-1$ and 3-9 DAC. Analysis was done with the application BiNGO of Cytoscape v3.4.0. P-value was adjusted for multiple testing by the Benjamini \& Hochberg FDR correction P-value $<0.001$.

Although the first dimension explained most of the variation, it will reflect also all genetic differences not directly related to regeneration rather than developmental regenerationrelated differences. Thus, to obtain first insight in the genes causing regeneration-related differences in overall transcriptional response, the "loadings" of the second dimension of the PCA axis, which separated 0-1 from 3-9 DAC, were used as query for gene ontology (GO) overrepresentation analysis. This analysis indicated that regardless the regeneration capacity of the explant, given by the genetic background or the position within the original bulb-scale (basal vs apical), transcripts related to "response to chemical stimulus", "response to inorganic substance", "response to wounding" and "developmental process" had a higher relative abundance in early time-points of regeneration (0-1 DAC) than in 3-9 DAC (Figure 2b). In later 
time-points (3-9 DAC), there was an upregulation of the terms "anatomical structure development", "DNA replication", "reproduction" and "cell cycle" (Figure 2b), which are expected terms for regeneration and meristem establishment.

To examine the regeneration process in more detail and to uncover the transcriptional differences among the four types of explants (GB, GA, PB, PA), we analysed the GO overrepresentation data at every subsequent time point compared to the experimental starting point (0 DAC). Transcripts under the term "response to chemical stimulus" re-appeared enriched in early time points, more specifically at 1 DAC for all types of explants. This was also the case for the term "response to hydrogen peroxide" (Figure 3).

Nevertheless, transcripts under the terms "organ development", "embryonic development", "post-embryonic development" and "cell cycle" were differentially enriched among the four type of explants, with a gradual response and enrichment at later time points in the poor regenerating explants (PA and PB) (Figure 3). This observation reveals a perfect correlation between the morphological and transcriptional responses, with initial a similar 'stress/wounding' response in all explant types, but subsequently, a differential response correlating with the observed differences in regeneration capacity and speed among the four types of explants (Figure 1d).

The remarkable observation that already three days after start of the culture a clear-cut transcriptional signature of regeneration and meristem initiation was visible, made us hypothesize that the initial triggers and signals of regeneration are probably already present at 1 DAC and this prompted us to investigate the early transcriptional response (0-1 DAC) in more detail.

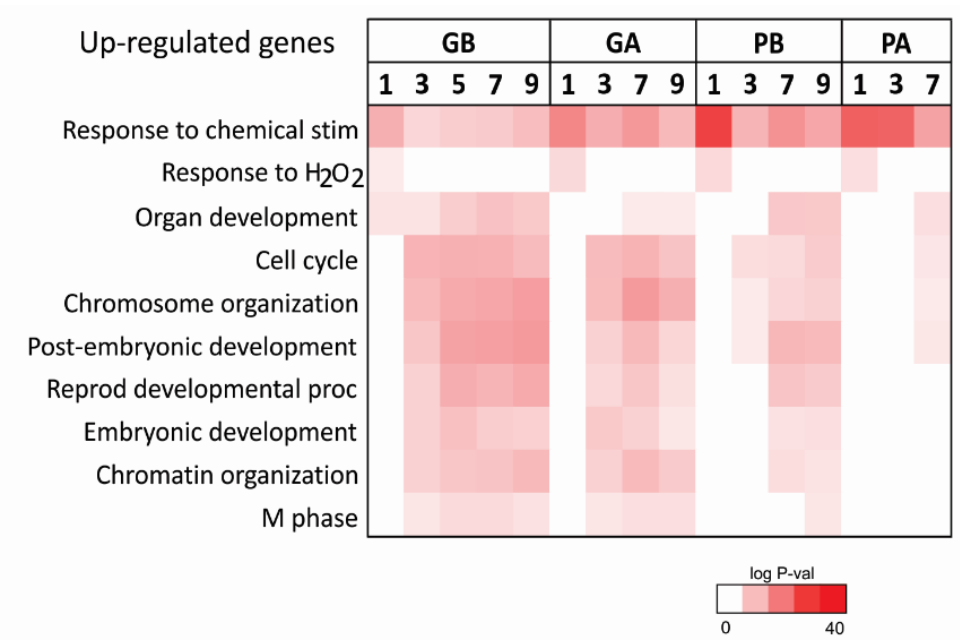

Figure 3. Gene Ontology overrepresentation analysis of biological processes differentially expressed at each time point of bulblet regeneration in comparison to the starting point (0 DAC). The analysis was done with the BiNGO application of Cytoscape v3.4.0 software. P-value was adjusted for multiple testing by the Benjamini \& Hochberg FDR correction P-value <0.001. GB: Good regenerating cultivar-basal explant. GA: Good-apical. PB: poor regenerating cultivar-basal explant. PA: Poorapical. 


\section{Early transcriptional responses within 1 DAC}

\section{Reactive Oxygen Species (ROS) response upon wounding}

The enrichment of GO terms related to "response to hydrogen peroxide" was present in all four explant types comparing 0-1 DAC, and mostly given by potential small heat shock proteins (sHsps) and peroxidases encoding transcripts (Figure 4a). It is well known that upon wounding of tissues a fast and transient ROS response can be expected (Minibayeva et al. 2015). However, since exogenous $\mathrm{H}_{2} \mathrm{O}_{2}$ has also proven to promote lateral (Li and Jia 2013) and adventitious root formation (Li et al. 2009), we hypothesised that the very efficient regeneration of lily without exogenous hormone treatments is caused by the high levels of peroxidases and that ROS might resemble an initial trigger for regeneration. Hydrogen peroxide $\left(\mathrm{H}_{2} \mathrm{O}_{2}\right)$ can be sensed by small heat shock proteins (sHsp) (Miller and Mittler 2006) and $\mathrm{H}_{2} \mathrm{O}_{2}$ levels are finetuned mostly by catalases and peroxidases. Unfortunately, the high number of lily transcripts coding for peroxidases and SHsp as well as their transient and variable expression did not allow to resolve whether ROS plays a role in the initiation of bulblet regeneration in lily (Figure 4a).

(a)

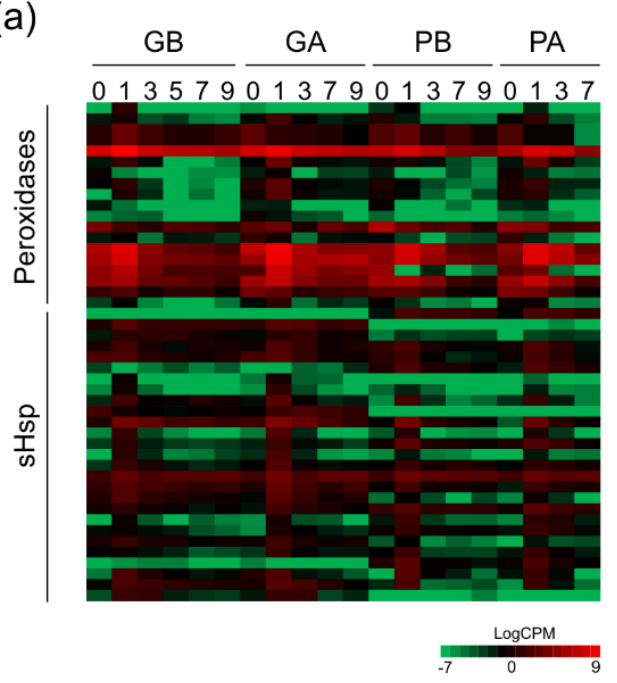

(b)

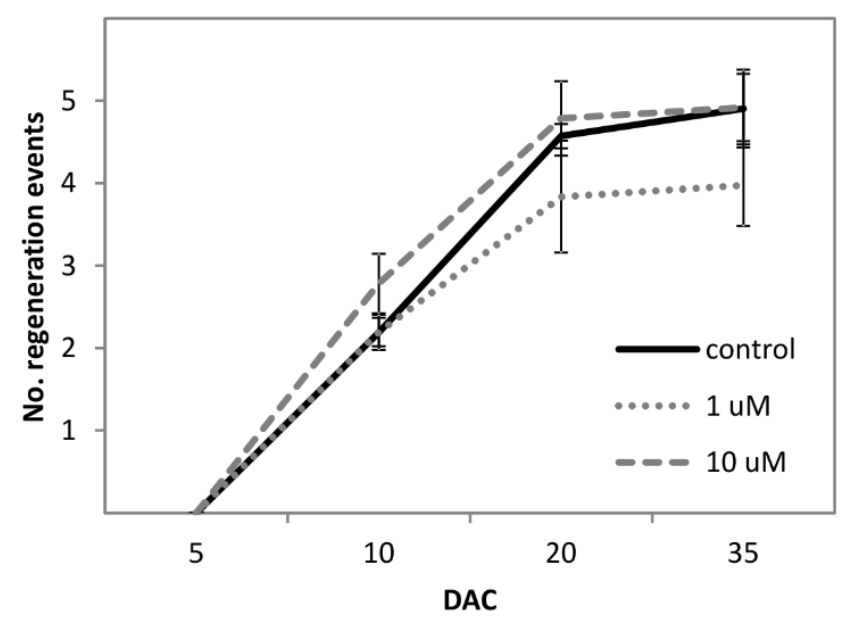

Figure 4. ROS response upon bulb scale detachment from the bulb. (a) Heat map of transcript expression of peroxidases and small heat shock proteins (sHsp) differentially expressed between early (0-1 DAC) and late (3-9 DAC). GA: Good regenerating cultivar-apical explant. GB: Goodbasal. PB: poor regenerating cultivar-basal explant. PA: Poor-apical. (b) Effect of the ROS scavenger Trolox on bulblet regeneration in Good regenerating basal lily scale explants (GB). Regeneration (' $\mathrm{D}$ '-stage or beyond) was scored upon culturing of explants in $1 \mu \mathrm{M}$ or $10 \mu \mathrm{M}$ of Trolox, or tap water as control. Explants were treated in moist chambers during five days and then transferred to normal incubation chambers with tap water. DAC: days after start of the culture.

To investigate a potential role for ROS in regeneration, we treated $G B$ explants with $\mathrm{H}_{2} \mathrm{O}_{2}$ and with Trolox, a water soluble $\mathrm{H}_{2} \mathrm{O}_{2}$ scavenger, and investigated the regeneration response. $\mathrm{H}_{2} \mathrm{O}_{2}$ treatment compromised the survival of the explants and therefore no data is available. In 
contrast, Trolox treated explants remained healthy, but did not show any effect on regeneration capacity $(\mathrm{P}<0.01$ ) (Figure $4 \mathrm{~b})$. Although we cannot rule out a positive effect of ROS on regeneration, we did not find any proof of it. The fact that Trolox treatment did not affect regeneration efficiency, suggests that the physiological response to cope with oxidative stress caused by wounding, is not having an effect on the regeneration process.

\section{Hormonal response upon wounding}

We then investigated early responses of hormone related genes from the GO term "response to chemical stimulus" (Figure $2 \mathrm{~b}$ and 3 ), since various studies assigned a role to hormonal signalling in the initiation of regeneration (reviewed in (Motte et al. 2014)). As expected based on the above described ROS-related wounding response, genes related to hormonal stress responses were identified. This included genes associated with e.g. abscisic acid (ABA), ethylene (ET), and jasmonic acid (JA) (Figure 5a and supplemental table 1). Moreover, genes supposed to be more closely related to the regeneration process, such as auxin (AUX) signalling and transport; cytokinin (CK) signalling and biosynthesis; and ET signalling related genes (Motte et al. 2014; Chatfield and Raizada 2008) were also found.

Among the differentially induced genes within one day after culture, ETHYLENE RESPONSE FACTOR 109 (LOERF 109), LOERF1 15 and WOUND-INDUCED DEDIFFERENTIATION 2 (WIND2) caught our attention, because of the positive role of ERF109 in auxin biosynthesis and of ERF115 and WINDs in organ regeneration (Cai et al. 2014; Heyman et al. 2016; Heyman et al. 2013; Iwase et al. 2011 a). Interestingly, most of the differences were given at 0 DAC, indicating that either these genes are activated already within 45 mins after excision (time used to disinfest the explants) or less likely, that those genes are expressed even when the bulb is at a physiological resting state. Intriguingly, at ODAC LOERF109 was higher expressed in PA explant, and LOWIND2 higher in the apical explants (Figure 5a).

Subsequently, we focussed on auxin and CK, because of their key roles in plant regeneration (Ikeuchi et al. 2016). Surprisingly, the most common rate limiting biosynthesis genes for both hormones, e.g. yucca flavin monooxygenases (YUC) and tryptophan amino transferases (TAA) for auxin (Zhao 2012); and ADENYLATE ISOPENTENYLTRANSFERASES (IPT) for cytokinin (Miyawaki et al. 2004), were not found in the early, neither late differentially expressed transcripts. Moreover, no significant changes in the levels of both hormones were found between 0 and 1 DAC (Figure 5b-c). Nevertheless, an auxin homeostasis gene, LoGH3.3, an auxin polar transporter PIN-LIKES (LOPILS7) and a CK activator from inactive CK, LONELY GUY 3 (LOLOG3) (Kurakawa et al. 2007), were higher expressed within the first day of culture, however at similar levels for all type of explants (Figure 5a). 
(a)

ABA degradation
AUX polar transport
AUX-activated signaling pathway
CK biosynthesis activation
CK-activated signaling pathway
CK and ET-activated signaling $\mathrm{p}$
ET-activated and JA-mediated
signaling pathway

ET-activated signaling pathway

GA-mediated signaling pathway GA and JA-mediated signaling $\mathrm{p}$ JA-activated signaling pathway

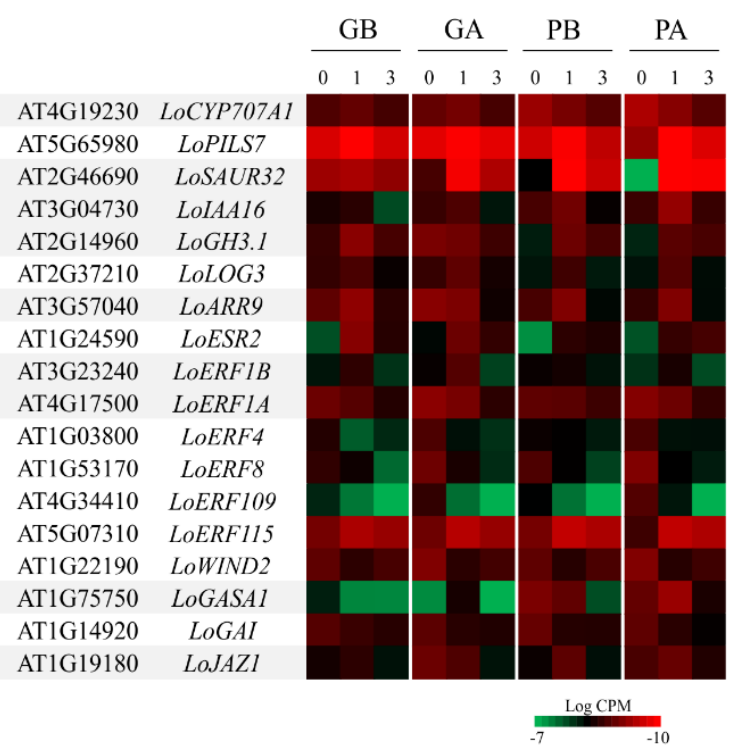

(b)

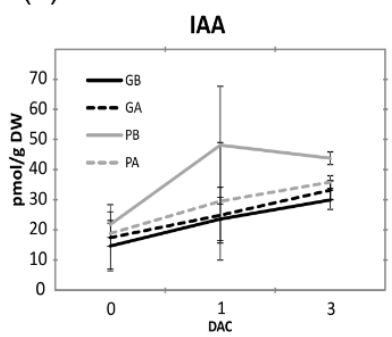

(c)

CK

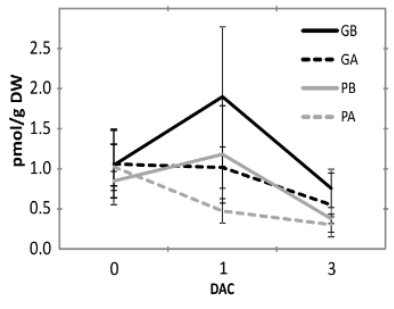

Figure 5. Expression of hormone related genes and endogenous levels of auxin and cytokinin during the first days of explant culture. (a) Early transcriptional activation of hormone related genes. Heat map of the expression levels was plotted for each type of explant at 0, 1 and 3 Days After the start of Culture (DAC). GB: Good regenerating cultivar and Basal explant, GA: Good-Apical, PB: PoorBasal, PA: Poor-Apical. ABA: Abscisic Acid. AUX: Auxin. CK: Cytokinin. ET: Ethylene. JA: Jasmonic Acid. GA: Gibberellic Acid. (b) Endogenous levels of active auxin (IAA). Although there was a trend of increase in auxin levels, there were no significant differences among the explants and between 0 and 1 DAC (P $\leq 0.05)$. (c) Endogenous levels of active CK (tZeatin and cZeatin). No significant differences among the explants and between 0 and 1 DAC were observed ( $\mathrm{P} \leq 0.05)$.

In summary, it seemed that lily bulblet regeneration is mediated by hormonal signalling upon wounding, affecting many hormone classes, among which JA, ethylene and auxin are most prominently present. Though this appeared not to be regulated at the level of de-novo hormone biosynthesis, but by changes in hormone transport, competence of tissues to respond to hormones and hormonal signalling cascades, resulting in the activation of bulblet regeneration.

\section{Transcriptional responses after 3 DAC}

The global RNAseq analysis (Figure 2) pointed to three days after culture as a kind of switch point and the moment that genes related to meristem establishment and de novo organogenesis got induced. To get a better understanding of the molecular processes occurring during this phase, we performed an in depth analysis of this transcriptional response.

\section{Cell proliferation}

GO terms related to cell proliferation were found to be enriched from 3 DAC in GB explants, whereas these terms were only found after 7 DAC in PA explants (Figure 3). This observation 
shows that the timing of activation of cell division related genes correlates with the observed differences in regeneration capacity and speed. Inspired by this, we further investigated the expression of all transcripts potentially directly related to the cell cycle (Supplementary Table 2). A heat map grouped with a similarity dendrogram in both axis corroborated the existence of several transcripts with high expression at 3 DAC and beyond for all types of explants (Figure 6a). A closer look into those transcripts revealed a small set of genes with no expression at 0-1 DAC for all explants, sUch as E2F TRANSCRIPTION FACTOR-LIKE (LOE2FL), whose expression was

(a)

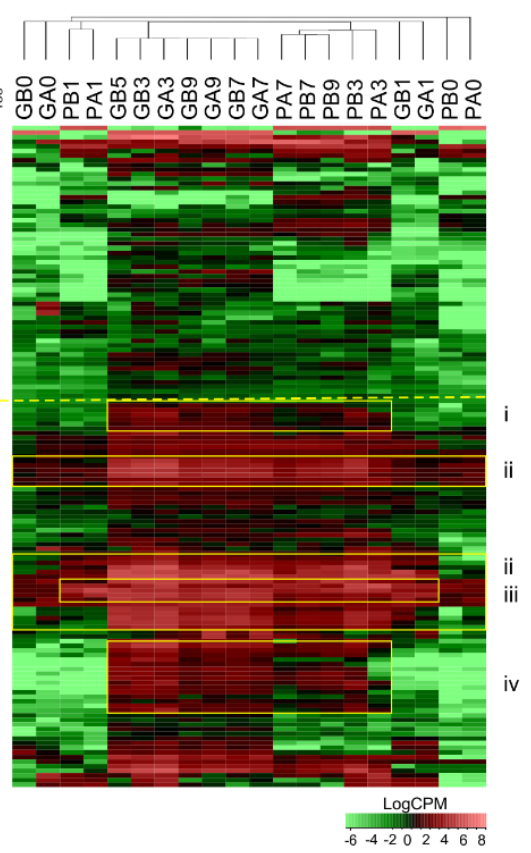

(b)

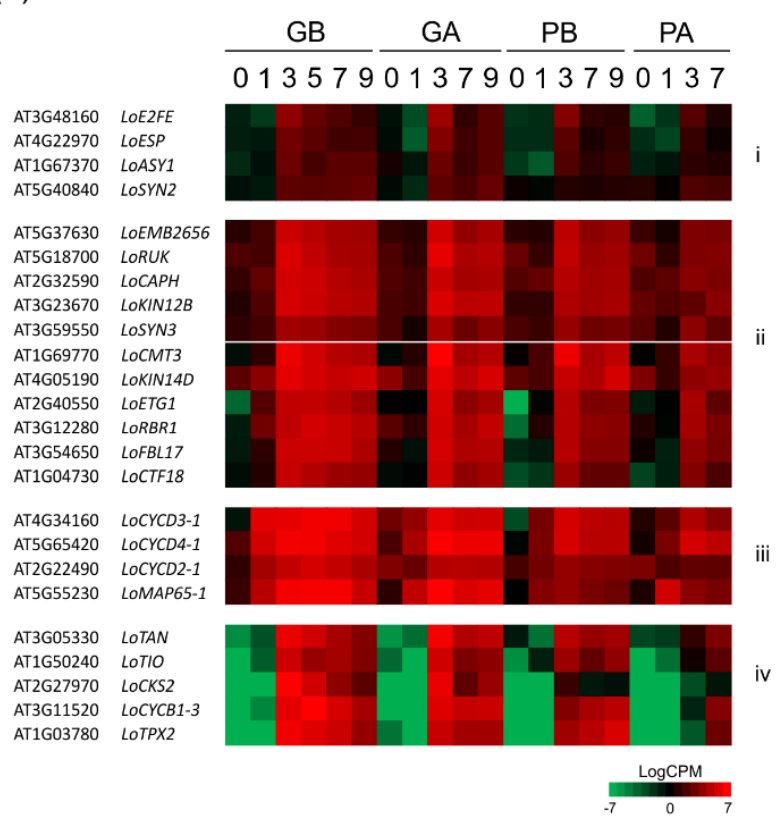

Figure 6. Heat map of differentially expressed transcripts from 0 DAC or between 0-1 and 3-9 DAC and involved in cell proliferation. This list of transcripts was obtained from GO over representation analysis (see figures 2 and 3). (a) Transcripts and explants were clustered based on Pearson correlation using the GeneMaths software. Dashed line separates the transcripts with low and high expression. Low expressed transcripts are mostly isoforms of the high expressed genes. (b) Detailed expression of transcripts with high expression, arranged by time and regeneration capacity. GA: Good regenerating cultivar-apical explant. GB: Good-basal. PB: poor regenerating cultivar-basal explant. PA: Poor-apical.

the lowest in PA explant (Figure 6b). Another set of genes showed moderate expression in early time points, while a significant up regulation at 3 DAC in GB, GA and PB explants (Figure 6b). The protein kinase RUNKEL (LORUK), E2F TARGET GENE 1 (ETGI) and the DNA (LOCYTOSINE-5)METHYLTRANSFERASE (LOCMT3) belonged to this group. Moreover, there was a small set of genes, mainly represented by D-type cyclins (LOCYCD2-1, LOCYCD3-1, LOCYCD4-1), which was highly induced at 1 DAC for all explants (Figure 6b). In line with the poor regeneration capacity of PA explants, there was a set of genes with no expression at early time points, but with a dramatic expression induction at 3 DAC for all explants except for PA, in which these genes 


\section{Chapter 5}

were induced later. Also, the overall expression level of these selected set of cell-cycle related genes correlated with their regeneration potential (e.g. higher expression in GB explants) (Figure 6b).

To get more detailed insight in cell-cycle progression of the scale explants and link this with the observed expression differences, we performed 5-ethynyl-2'-deoxyuridine (EdU) staining to identify the cells in which DNA synthesis occurred. This analysis showed that DNA synthesis started already at 1 DAC in GB explants (Figure 7a-c), corroborating the expression of D-type cyclins shown in Figure 6b. Moreover, DNA synthesis was activated randomly in parenchymatic cells, but this was progressively concentrated towards the adaxial side of the explant (Figures $7 d-e)$. The subsequent progression from $S$ to $M$ resulted in a population of new small and asymmetrical cells lacking the typical starch granules (Fig. 7f), suggesting cell dedifferentiation followed by active proliferation. Finally, the cell divisions became more localised and oriented, resulting in the formation of a pro-meristem from the population of dedifferentiated cells in the proximal-adaxial side of the explant (figure $7 f$ ).

\section{Organ development and post-embryonic development}

Transcripts in GO terms "organ development", "embryonic development" and "postembryonic development" appeared to be enriched from 3 DAC in GB explant, whereas only at 7 DAC for the poor regenerating PA explants (Supplementary table 3). In order to get a better view, we investigated which genes are exactly covered by these GO categories. As expected, some genes with pivotal roles in meristem formation were present in these GO categories and differentially expressed according to the regeneration capacity of the explant, including ETHYLENE-RESPONSIVE TRANSCRIPTION FACTOR 2 (LOESR2) and SHOOT MERISTEMLESS (LOSTM). Moreover, both these genes, and a WUSCHEL-RELATED HOMEOBOX 13-like gene (LoWOX 13), had not any expression detected at O DAC, while their expression was significantly induced in GB explants and much earlier and at higher levels in GB in comparison to PA explants later during the regeneration process (Figure 8a-C). Expression of AtWOX 13 has been observed in the shoot apex (Schmid et al. 2005), but its function there is still elusive. A sequence similarity analysis revealed the close homology of LoWOX13 with several WOX13-like genes in monocots species, but also with Arabidopsis WOX10 and WOX14 (Supplemental figure 1). Remarkably, the expression of LoWOX 13 is already peaking at 1 DAC in GB explants. 

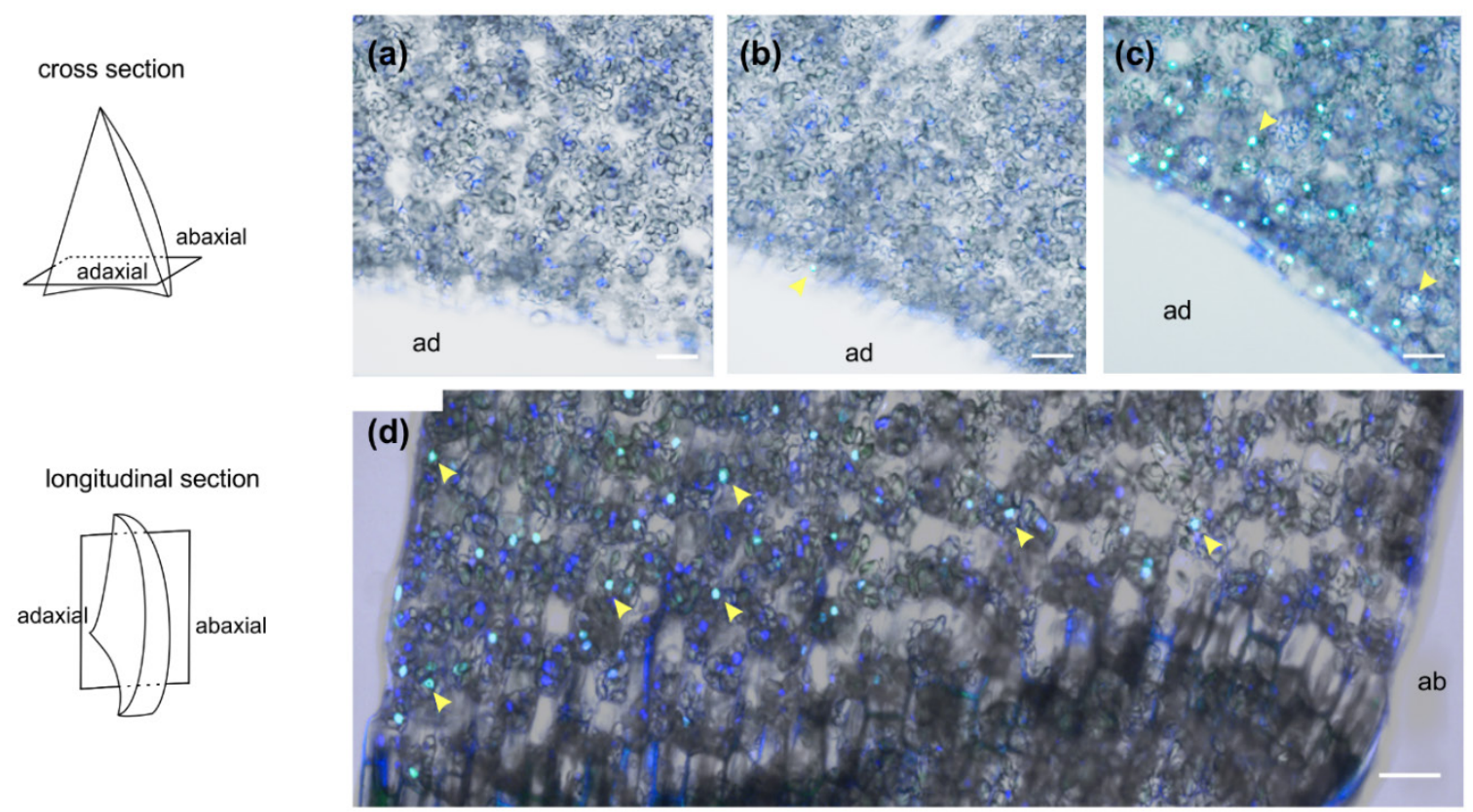

longitudinal section
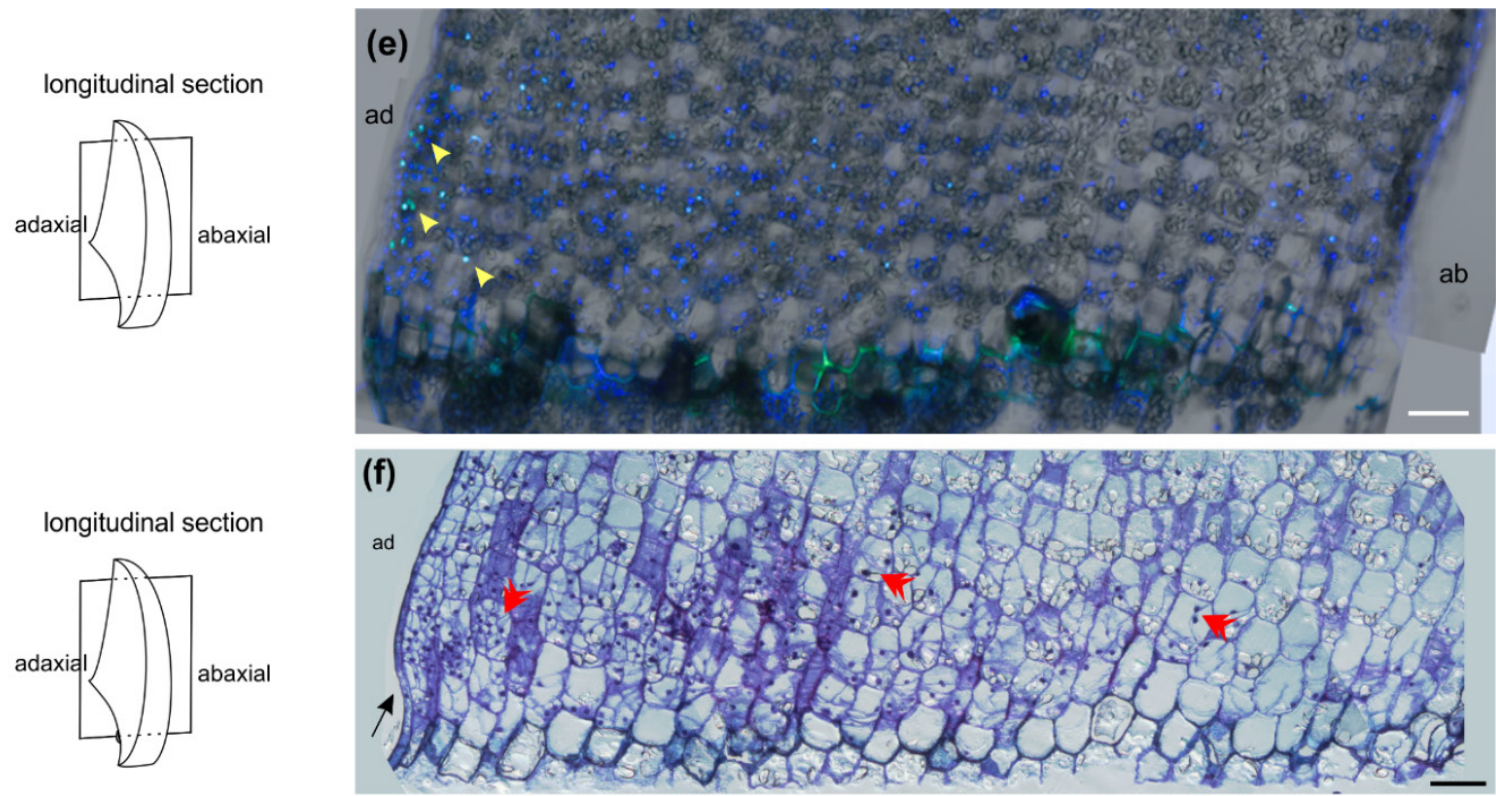

Figure 7. DNA synthesis and cell proliferation pattern upon explant detachment assessed by fluorescent and light microscopy. Edu was incorporated in DNA of the nuclei going through the Sphase of the cell cycle (staining for 12 hours prior to imaging). Yellow arrow heads: nuclei that incorporated EdU. Scale bar: $200 \mu \mathrm{m}$. Ad: Adaxial. Ab: Abaxial. (a) Cross section representative of the GB explants at 0 days after culture (O DAC) stained with Edu and DAPI. The lack of Edu signal indicates that no cells have started DNA synthesis at this time point. (b) Cross section of GB at 1 DAC. Edu signal is observed in one cell nucleus at the adaxial side of the explant. (c) Cross section of $G B$ at 3 DAC. Several adaxial parenchyma cells have undergone DNA synthesis. (d) Representative longitudinal section of GB explants at 5 DAC showing that DNA synthesis occurs at high frequency in the adaxial parenchyma cells. (e) Representative longitudinal section of GB explant at 7 DAC. Edu signal indicates that DNA synthesis is now more restricted in the parenchyma cells in close vicinity to the epidermis. DAPI signal indicates a higher number of nuclei at the adaxial parenchyma cells than at the abaxial region. (f) Cell proliferation in a representative longitudinal section of GB explant at 9DAC, right at the place where a pro-meristem is arising (indicated by the arrow). Toluidine Blue staining was used. Red arrow head points at parenchyma cells where several rounds of cell division took place, resulting in very small, asymmetrical and starch less daughter cells. 
Another group of genes potentially related to meristem formation, included two genes coding for a CUP-SHAPED COTYLEDON3 (CUC3) member and HOMEOBOX PROTEIN KNOTTED-1-LIKE 6 (LOKNAT6). Expression of these genes significantly increased in GB explants from 1 DAC onwards, but remarkably, had an initial sharp down regulation at 1 DAC in the less-well regenerating apical explants (Figure $8 \mathrm{~d}-\mathrm{e}$ )). This observation reveals a correlation between the level of expression of LOCUC3 and LOKNAT6 at 1 DAC and the regeneration capacity of the explants. As for LoWOX13, also the expression of these genes already sharply increased in GB explants at IDAC, although they reached their maximum expression level at 3-7 DAC.

Interestingly, a set of genes previously associated to pro-meristem formation was already expressed at the start of the culture (ODAC). These genes got further induced from 3 DAC onwards and there were no differences in level or timing for expression among the explants (Figure $8 \mathrm{f}-\mathrm{i}$ ). Therefore, this set of genes seems to play an important role in all type of explants but not to be causal for the observed differences in regeneration potential (Fig. 1). Examples are TOPLESS (LOTPL); ARGONAUTE 1 (LOAGOI); AUXIN SIGNALING F-BOX 3 (LOAFB3); and SWINGER (LOSWR). TPL is known to modulate auxin signalling and to repress root-specifying genes during shoot regeneration (Chatfield et al. 2013). Argonaute proteins are key players of small-RNA-mediated gene silencing (Meister 2013) while SWR mediates silencing through DNA methylation.

\section{Transcriptional clusters reveal insights in acquisition of shoot regeneration capacity}

Based on the above detailed analysis of gene expression and the observed expression response of e.g. LOSTM, which is a well-known marker for shoot meristem identity (Long and Barton 2000), we hypothesized that the acquisition for shoot meristem competence must be obtained within the first day of culture (0-1 DAC) in the best regenerating explants (GB), while delayed with at least 2-6 days in the poor regenerating explants (PA). We argued that genes co-expressing with LOSTM in GB explants would be good candidates for genes potentially involved in lily regeneration. Therefore, we performed a clustering analysis to identify all genes co-expressing with LOSTM. We restricted the search to transcripts with a BLAST hit to Arabidopsis genes previously reported to behave as common markers of root-callus-leaf (RCL) regeneration competence (Sena et al. 2009), genes that mark unique shoot apical meristem regions (Yadav et al. 2009; Yadav et al. 2014), and genes known as markers for root identity (Brady et al. 2007). In this way, we aimed to select against those genes with a similar expression pattern, but e.g. linked to the wounding and general stress response. The same clustering approach was also used to identify groups of genes, whose expression is closely associated to the different developmental and morphological phases of the regeneration response defined in Figure 1. 
(a)

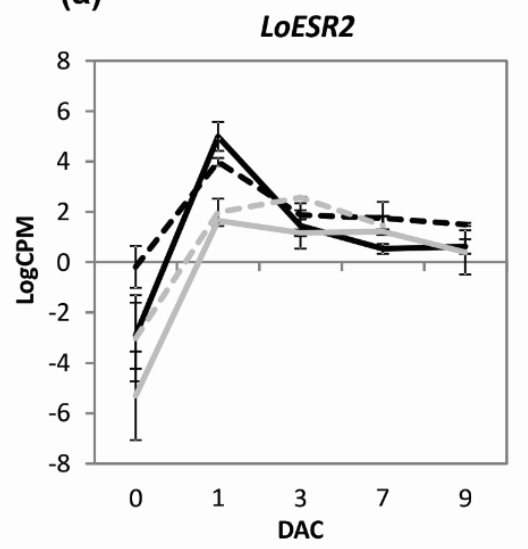

(d)

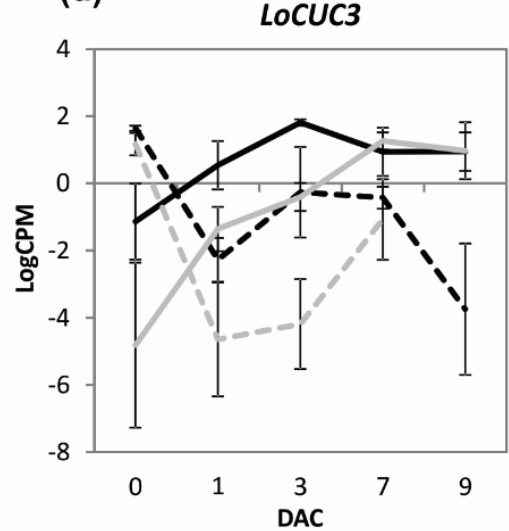

(g)

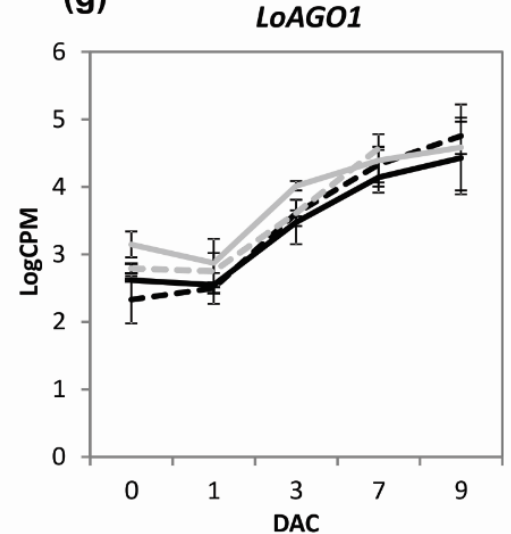

(b)

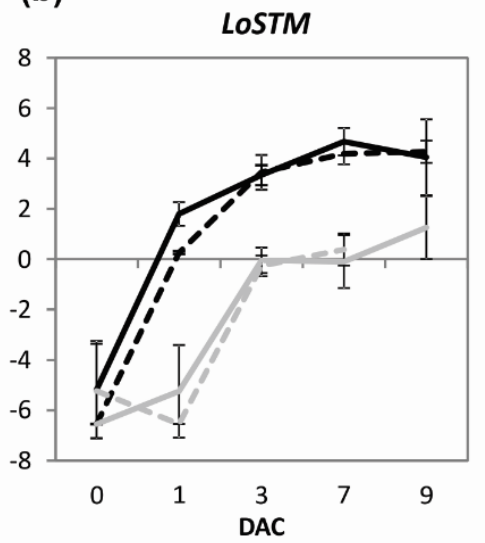

(e)

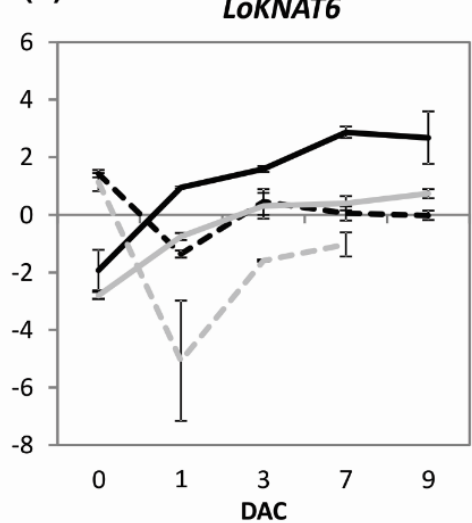

(h)

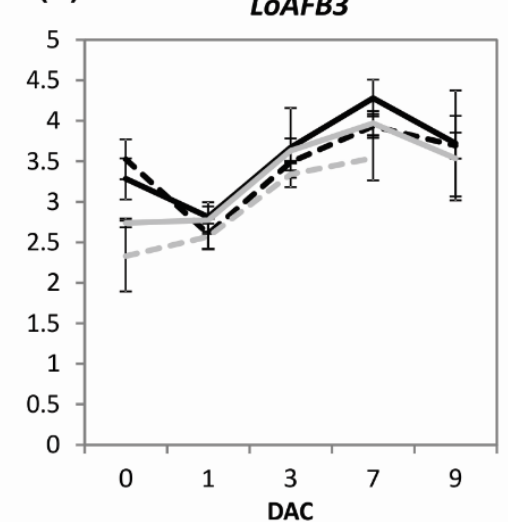

(c)

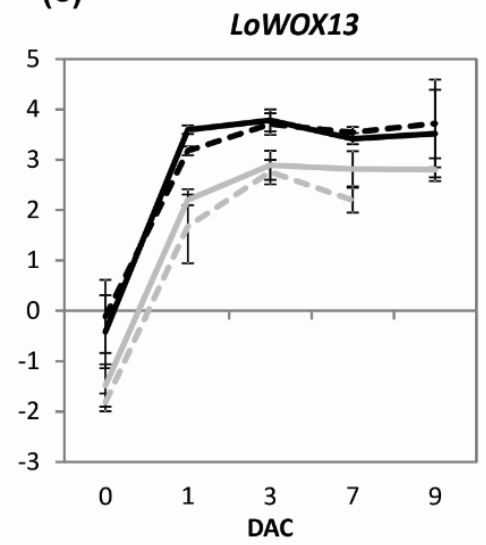

(f)

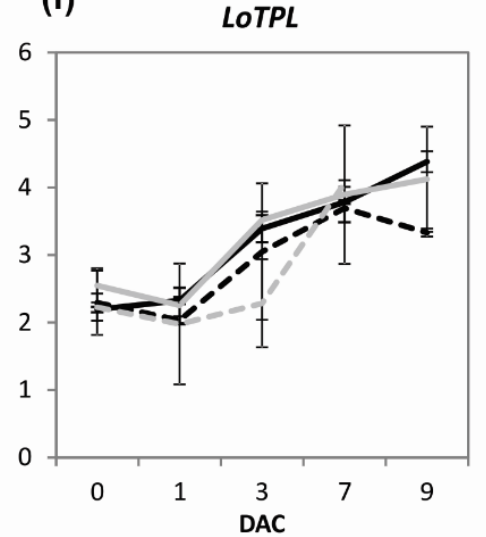

(i)

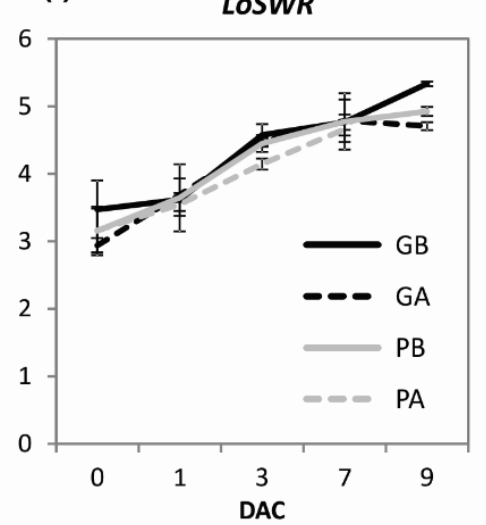

Figure 8. Transcriptional changes of genes related to meristem formation. (a) Expression of LOESR2. (b) Expression of LOSTM. (c) Expression of LOWOX13 (d) expression of LOCUC3. (e) Expression of LoKNAT6. (f) Expression of TPL. (g) Expression of LOAGO 1. (h) Expression of LOAFB3. (i) Expression of LOSWR. GB: Good-basal explant. GA: Good-apical. PB: Poor-basal. PA: Poor-apical. DAC: days after culture.

To mine the huge transcriptome dataset, the Self-Organizing Map (SOM) clustering method was applied on the GB explant RNAseq dataset. Nine groups were obtained, from which SOM1 
showed a pattern expected for repressors of regeneration, given its high expression at 0 DAC followed by a progressive down regulation. The pattern of SOM4 and SOM7 suggested an activation role at 1 or 3 DAC; and SOM 8 and SOM9 resembled a meristem-like pattern, because of the presence of LOSTM in SOM8, the overall timing of expression induction, and subsequent maintenance of the expression levels (Figure 9a).

In general, almost half of the reported root and shoot identity and RCL regeneration competence markers could be found in our lily transcriptome (supplemental table 4), suggesting conservation of the regeneration process and that bulblet regeneration in lily shares many transcriptional features with root, shoot, and callus formation, and regeneration in model dicot plants, such as Arabidopsis. Nevertheless, we did e.g. not detect expression of WUSCHELRELATED HOMEOBOX 5 (WOX5), a key gene in the maintenance of the stem cell niche of the root meristem (Pi et al. 2015) and a marker for the initial stages of callus regeneration (Atta et al. 2009; Sugimoto et al. 2010).

Based on the fact that the top two highest percentages of selected marker genes mapped into SOM8 and 9, including LOSTM, we believe that these two clusters of genes are the most relevant for lily regeneration (Figure 9b). Besides that, a relative high percentage of shoot identify markers mapped into SOM4, which shows a peak of expression at 1 DAC (Figure 9b). Hence, this cluster (SOM4) is supposed to contain the initial, but transient inducers of regeneration and meristem initiation.

Subsequently, we decided to investigate the behaviour of the marker genes contained in the abovementioned clusters, in the two basal explant types. Although these two types of explant aren't resembling the biggest difference in regeneration capacity, they show a different expression response in timing and are both originating from the same location in the scale and in that respect the most similar. Many marker genes from SOM8 and SOM9 were differentially expressed between the two explants and their expression correlated with the explant regeneration capacity (supplemental table 4), sUch as EARLY NODULIN-LIKE proteins (LOENODL); LATERAL ROOT PRIMORDIUM 1 (LOLRPI); HOMEOBOX GENEI (LOATHI), LOSTM and DEFECTIVE IN CUTICULAR RIDGES (LODCR) (Figure 9c-f). Surprisingly, most of the transient shoot markers from SOM4 were not differentially expressed between the two basal explant types (supplemental table 4). 
(a)
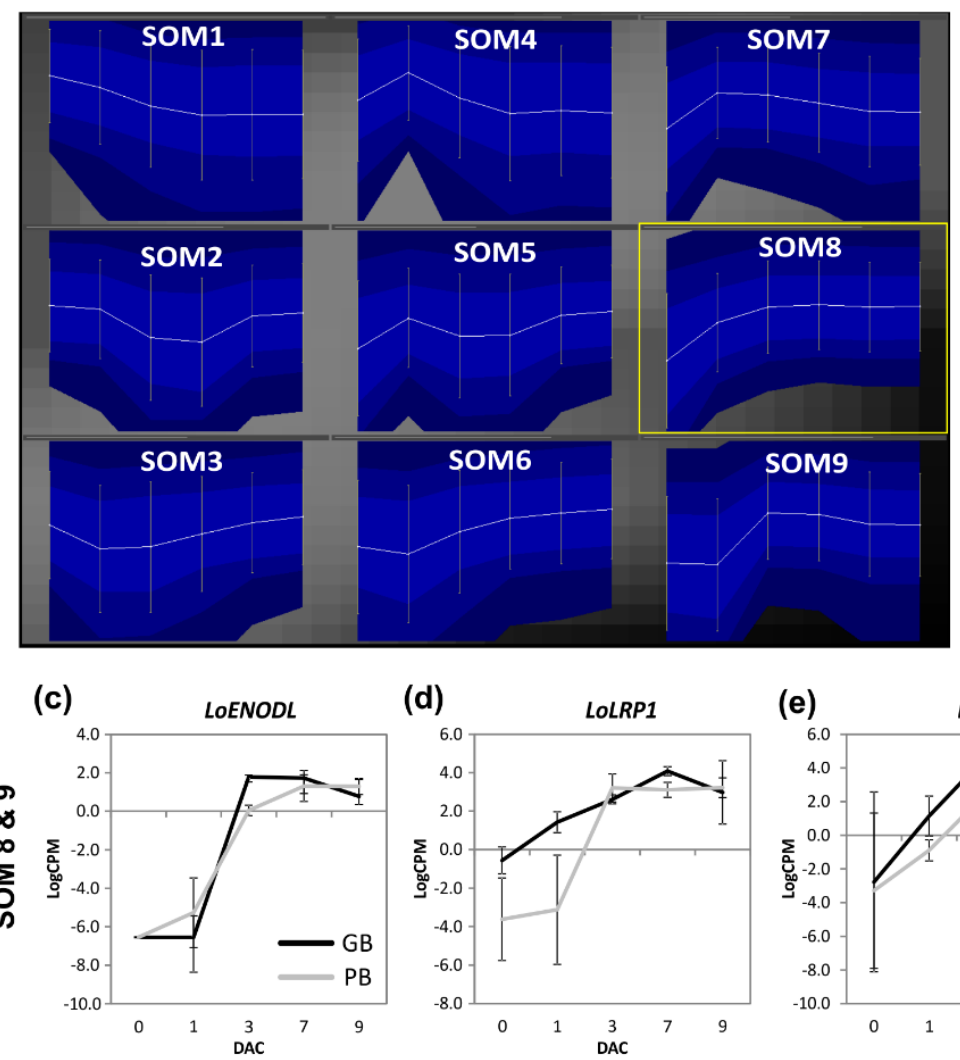

(d)

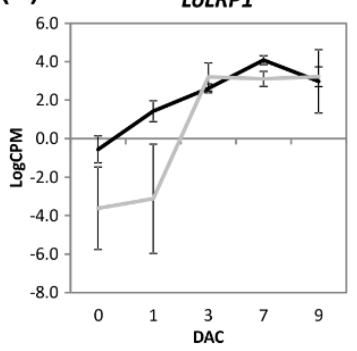

(b)

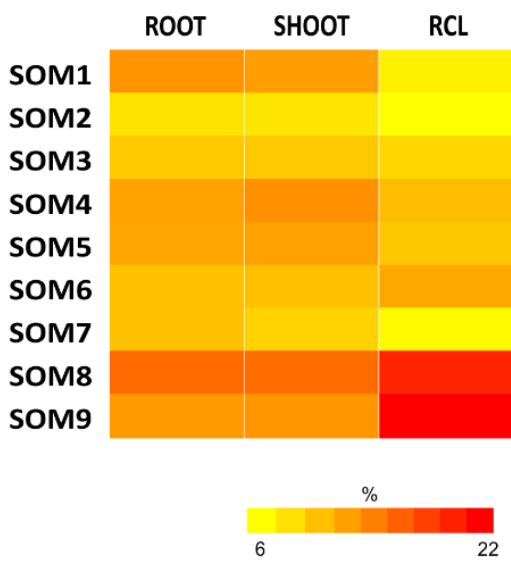

(e)

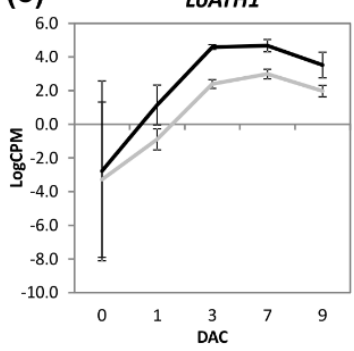

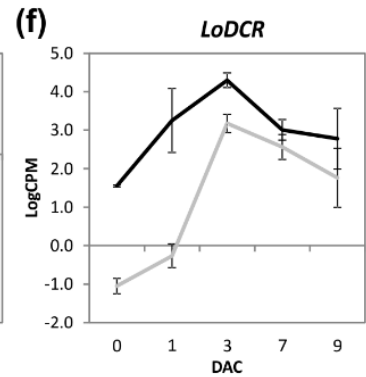

Figure 9. Transcriptional clustering and distribution of regeneration competence markers in the explant with best regeneration potential (GB). (a) Transcriptional clusters of GB explants. Nine clusters were obtained using the Self Organizing Map (SOM) clustering method with Pearson correlation and a matrix of $3 \times 3$. LOSTM clustered in SOM8 (highlighted in yellow). (b) Heat map representing the percentage of potential root identity, shoot identity or root-callus-leaf (RCL) regeneration markers contained in the nine SOMs. Based on the top three highest percentages, root markers were mostly contained in SOMs 8, 9 and 1; shoot markers in SOMs 8, 9 and 4; and RCL regeneration potential markers in SOMs 8, 9 and 6. (c-f) Examples of transcripts from SOM8 and SOM9, which follow an STM-like pattern of expression. (c) Expression pattern of LOENODL. (d) Expression of LOLRP1. (e) Expression of LOATH 1. (f) Expression of LODCR 1. GB: bood-basal explant. PB: Poor-basal explant. DAC: days after the start of culture.

\section{Discussion}

Lily is a bulbous plant species with an enormous regeneration capacity. Upon detachment of lily bulb scales, bulblets with a fully organized SAM and three to four scales are formed at the adaxial base of the scale explant within forty days. Surprisingly, the wound-induced initiation of regeneration in these scale explants seems to occur at tremendous speed and within 24 hours after explant excision for all investigated explant types. Thus the differences in timing of promeristem formation between different explant types might not necessarily be reflected by transcriptional differences in the first hours or days after explant excision. This fact makes it more complicated to dissect the true initial regeneration triggers from the general wounding response occurring in all explant types. 
Upon detachment from the mother bulb, the lily explants initiate a series of physiological responses to recover from tissue wounding, including activation of defence genes and induction of ROS scavengers. Those processes are known to be mediated by the plant hormones ethylene, salicylic acid, abscisic acid, and jasmonic acid (reviewed in (Bartoli et al. 2013)). ROS production upon wounding appears to trigger regeneration in animals, e.g. salamander (Godwin et al. 2013) and Xenopus (Gargioli and Slack 2004); as well as in plants, e.g. during lateral and adventitious root formation in Arabidopsis (Li and Jia 2013; Li et al. 2009). Although we detected an early induction of genes related to ethylene and jasmonic acid signalling pathway, we did not find an effect of ROS modulators on the regeneration capacity of lily explants. This finding is in line with a previous study attempting to correlate regeneration capacity of tulip bulb scales with their redox state given by ROS after wounding. Their results did not point at oxidative stress as mediator of regeneration capacity (van Rossum 1997). However, the regeneration response upon wounding appears to be extremely fast, and taking into account the complexity of ROS signalling (Foyer and Noctor 2005), we cannot conclude based on our observations and experiments that ROS doesn't play a role as triggering signal for bulblet regeneration.

\section{APETALA2/Ethylene Responsive Factor (AP2/ERF) transcription factors as activators of regeneration in cross-talk with hormones}

Recent discoveries point at some ethylene responsive factors (ERFs) as integrators of stress related hormones with developmental processes, involving the activation of cell division, giving rise to regeneration and organ formation (Licausi et al. 2013). For instance, ERFI and ERF109 mediate cross-talk between JA signalling and auxin biosynthesis in relation to lateral root elongation and formation, respectively (Mao et al. 2016; Cai et al. 2014). Although LoERF1 and LOERF109 were induced within the first day after culture, we did not detect induction of auxin biosynthesis genes but rather of genes related to polar auxin transport, such as LOPILS7. This observation, together with no significant increase of active IAA in the explants after $24 \mathrm{hr}$ of culture, suggests that excised lily bulb scales use their existing pools of auxin to create the required gradients to initiate meristem regeneration. The negative correlation of LOERF109 with the explant regeneration potential suggests that good regenerating explants are more sensitive to JA and auxin. Related to these observations, it would be interesting to test whether LOERFs can mediate the transcriptional activation and or polarization of auxin transporters and as such, the correlation between ERFs expression level and regeneration capacity.

In addition to ERFI and ERF109, ERFI15 in Arabidopsis roots promotes cell division and regeneration in cells in close vicinity to wounded cells, granting them the ability to regenerate and replace the damaged cells (Heyman et al. 2016). In our experimental system, a lily gene 
with high percentage of similarity to Arabidopsis ERF 15 was highly expressed within the first day of culture in all explant types. In Arabidopsis, ERF 115 induction of expression has been reported to precede stress activated cell division (Heyman et al. 2016), and in our system, cell proliferation boosted at 3 DAC. Therefore, it is tempting to speculate that ERF 115 in lily also mediate a hormonal cross talk to induce ectopic meristems in lily scales, although more research is needed to confirm this hypothesis.

Besides ERFs, another gene annotated as an ethylene-activated signalling pathway in Arabidopsis (www.arabidopsis.org), WIND 1 , is induced at the excision lines of wounded tissues, and it promotes dedifferentiation through the formation of callus (Iwase et al. 2011 a). LoWIND2, a homolog of WINDI was induced very early upon excision and its expression pattern resembled the one of WINDI reported by Iwase et al 2011, highlighting the possible role of the LOWIND2 protein as an integrator of the wound-induced signals and regeneration. In fact, ectopic expression of ERF 115 in Arabidopsis activates WIND 1 expression and neoplastic growth (Heyman et al. 2016). The exact mode of action of WIND genes and their downstream targets are not completely elucidated yet, but it has been proposed that WINDs enhance endogenous cytokinin responses to promote cell dedifferentiation (Iwase et al. 201 la; Iwase et al. 201 lb). Intriguingly, exogenous cytokinins do not promote regeneration in lily bulb scales (Van Aartrijk and Blom-Barnhoorn 1981), and no significant changes in the endogenous levels of active cytokinin was observed in the explants 24hr after wounding. However, the early (IDAC) induction of LOESR2, an ERF gene activated upon high cytokinin to auxin ratio, and a promoter of shoot regeneration (Matsuo et al. 2011), suggests that cytokinin responses might occur very fast, before 1 DAC. Therefore, we might have missed the expression of key regeneration triggering genes since no time points before 1 DAC were included in our analyses. Hence, it would be of great interest to investigate the possible roles of these selected AP2/ERF genes as early wound-induced regeneration genes in lily bulb scales.

It is important to note, however, that although ethylene regulates the expression of several ERFs and this is how this class of APETALLA2-like transcription factors obtained their name (Nakano et al. 2006), they are not always all and only responsive to ethylene. In Arabidopsis, ERF109, and ERF 115 are more responsive to JA and brassinosteroids (BR), respectively (Heyman, Cools et al. 2013, Cai, Xu et al. 2014). Hence, not only auxin and cytokinin might play a stimulating role during lily bulblet regeneration, but it is very well possible that stress hormones such as JA and ethylene play a role as well, as was proposed previously for ethylene (Chatfield and Raizada 2008).

Although wounding is necessary to trigger regeneration in lily bulb scales, it is intriguing that regeneration was never observed in the distal side of the explants, where there is also an excision wound. This basipetal gradient of regeneration also occurs in leaf segments of begonia and Saintpaulia ionantha and has been linked to differences in auxin levels in the leaf 
(Schraudolf and Reinert 1959; Kukułczanka and Suszyńska 1972). However, the endogenous levels of active IAA did not proof to be different in basal and apical lily explants. In Arabidopsis roots, wounding of the tip rapidly changes the auxin distribution within 16 hours, creating a new auxin maximum, which results in de novo root meristem regeneration (XU et al. 2006; Sabatini et al. 1999). Therefore, we propose that in lily scales, wounding also causes an auxin transport disruption, which results in several points of auxin maxima along the proximal side of the explant, which is the side with highest endogenous auxin levels. At the same time, the new auxin maxima points might induce asymmetrical cell proliferation, resembling a cell dedifferentiation process. The auxin re distribution might be in turn mediated by the action of an ERF transcription factor, such as LOERF109.

\section{Cell fate re-programming}

The correlation between the expression of genes related to the transition of G2 to M phase of the cell cycle, and bulblet regeneration capacity, highlighted the importance of massive cell proliferation prior to meristem initiation. We speculate that this massive but organized and localized cell proliferation upon explant detachment is required to re-program the cell fate of the parenchymatic tissue and to acquire their competence to regenerate shoots. In Arabidopsis, this competence is acquired during callus formation when CUC2 expression is induced (Kareem et al. 2015; Motte et al. 2011). Although we predicted that the competence to regenerate shoots was acquired before the expression of the shoot meristem marker STM, our data indicated that a high percentage of regeneration competence markers coexpressed with LOSTM. Moreover, the expression of CUC3, a gene partially redundant to its homolog CUC2 (Vroemen et al. 2003), did not always precede STM expression. This could be caused by asynchronous bulblet regeneration events taking place in the explant, which masked the real timing of particular transcriptional events. Otherwise, the acquisition of regeneration competence in lily bulb scales might not depend on CUC expression, or subsequent steps in the regeneration processes follow each other fast in a few hours of time and therefore, are missed due to our daily sampling schedule.

The high number of root and shoot identity markers and RCL regeneration competence markers found in our data indicate that de novo lily organogenesis shares many transcriptional features with shoot, root and callus regeneration in well studied dicot model plants. In fact, it has been reported that shoot and lateral root formation in Arabidopsis share their initial developmental stages (Atta et al. 2009; Sugimoto et al. 2010). The correlation between regeneration capacity of the lily explants and the timing and level of expression of genes related to root organ formation, such as LOLRPI, support this idea. Additionally, the expression of SCR, a gene involved in positioning the QC in the root meristem (Sabatini et al. 2003), but 
also a marker in the early stages of callus regeneration, raises the question whether lily explants go through an initial and transient callus-like state. Independent from this question, SCR in Arabidopsis also controls asymmetrical cell division (Moreno-Risueno et al. 2015) and its expression has been detected before in the shoot apex of Arabidopsis (Schmid et al. 2005). Therefore it would be interesting to investigate the role of LOSCR during bulblet regeneration. Last, given the correlation between regeneration capacity and some genes not reported to date to be involved in meristem regeneration but belonging to gene families whose members do play a pivotal role in this process, e.g. LoWOX 13 and LOCUC3, it is worth investing more time and resources in deciphering their function.

\section{Proposed model of lily bulblet regeneration from bulb scales}

Based on the expression of some key genes reported to play pivotal roles in regeneration in model species, it was clear that LOERF115 and LoWIND2 are induced extremely fast upon explant excision, and this induction appeared to occur in all explants regardless their regeneration capacity (Figure 10a). Moreover, induction of D-type cyclins (DNA synthesis) are very fast in explants with good regeneration capacity. Within one day after explant excision, LOESR2 and LOWOX13 are induced in all explants but their levels of expression depends on the regeneration capacity of the explant. Next, LOSTM is induced and its expression and timing depend on the regeneration capacity of the explant. Then a boost of cell proliferation takes place reflected by the differential expression of LOCYCBI-3 according to the regeneration capacity of the explant, and from this point onwards pro-meristem formation arises. The expression of these genes in relation to the initial steps of regeneration is schematically summarized for GB and PB explants in Figure 10b. This proposed model for bulblet regeneration in lily bulb scales starts with triggering the activation of $A P 2 / E R F$ transcription factors by wounding, which subsequently activate polar auxin transport re-distribution and cell proliferation and de-differentiation, to finally give rise to expression of genes such as LOSTM and pro-meristem establishment (Figure 10b). 
(a)
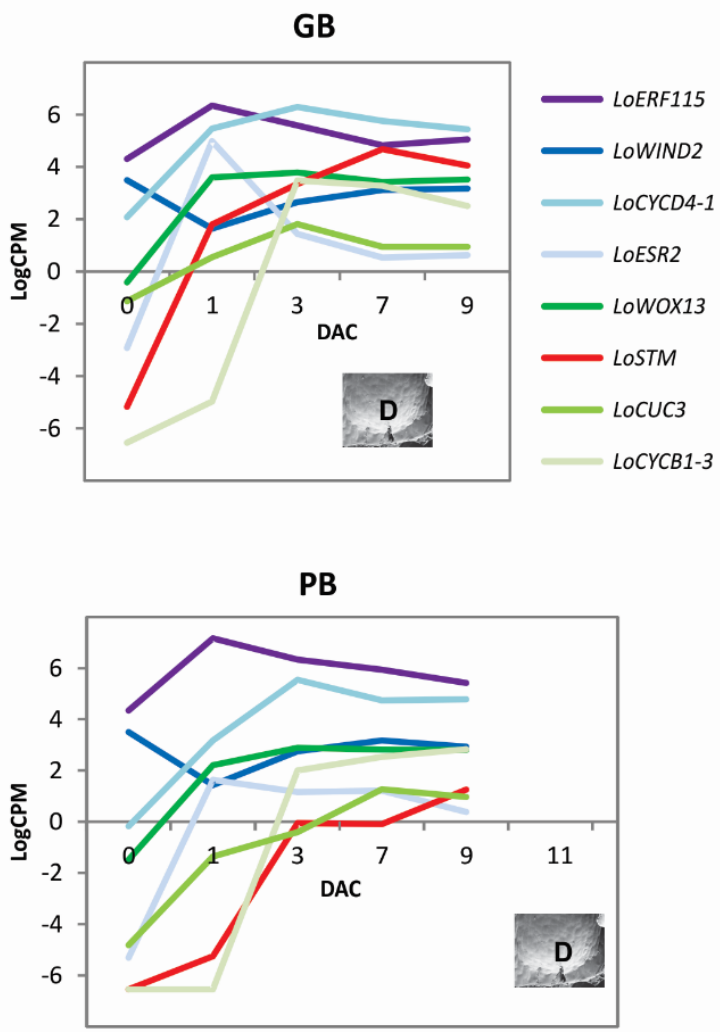

(b)

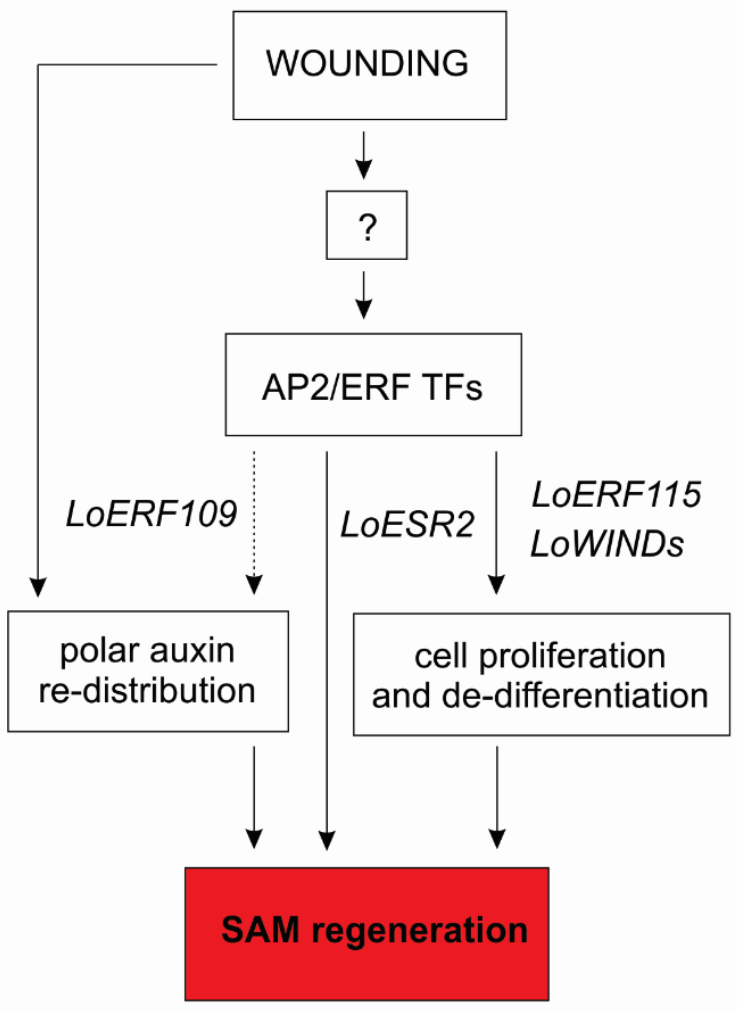

Figure 10. Summary of gene expression and molecular pathway underlying ectopic bulblet regeneration in lily bulb scales. (a) Transcriptional changes upon explant excision and culture in two basal explants differing in regeneration capacity given by the time of appearance of first ectopic regenerated pro-meristems and number of them. (b) Proposed model of ectopic bulblet regeneration in lily bulb scales. Wounding must trigger a physiological response (e.g. ROS) which activate several AP2/ERF transcription factors. Polar auxin re-distribution might be triggered by wounding itself or could be mediated by ERFs, such as LOERF109. Cell proliferation and dedifferentiation can be mediated by the rapid induction of LOERF115 and LOWIND2. In turn, the competence to regenerate shoots could be acquired by LOESR2, followed by activation of LOSTM and other meristem marker genes.

\section{Conclusion and Perspectives}

This study constitutes the first step in understanding the molecular control of de novo SAM regeneration by an RNAseq-based transcriptomics approach. A challenge remaining will be to identify the few key elements that are at the start of the process and responsible for the differences in regeneration speed and efficiency. Our approach revealed that many potential regeneration markers show a similar response in the different explant types, irrespective of their clear cut difference in regeneration response. This result suggest, that these differences are not due to a complete lack of capacity to respond, but because of slight differences in timing of activation or the lack of a few essential factors. Finding these needles in the haystack will be a challenging task for follow up studies. 


\section{Materials and Methods}

\section{Plant material and culturing conditions}

Apical and basal explants of mid located lily bulb scales (Figure la) of cultivars Paradero, McAleese, and $\mathrm{C} 15$ were cultured on a germination table with flowing tap water at $23^{\circ} \mathrm{C}$ and a 12/12 hr day/night light rhythm. In short, the explants laid standing up-right on a wet filter paper, which is in contact with the flowing warm water. Every island of filter paper containing explants was covered by a transparent plastic cup, which provided a moist and warm environment (Figure 1a). Five explants were collected at $0,1,3,5,7$, and 9 days after start of the culture (DAC), and observed under a stereomicroscope to count the number and stage of regeneration events, following the developmental stage classification of Marinangeli et al 2003 (Marinangeli et al. 2003). The most proximal region of the explants ( $5 \mathrm{~mm}$ thick) was collected in liquid nitrogen, freeze dried, ground into powder, and immediately stored at $-80^{\circ} \mathrm{C}$ for posterior RNA isolation.

To investigate the potential effect of Reactive Oxygen Species (ROS) on regeneration capacity, basal explants of $\mathrm{C} 15$ were cultured in moist chambers with 5 Whatman filter papers (Sigma-Aldrich) soaked with 1 or $10 \mu \mathrm{M}$ of 6-hydroxy-2,5,7,8-tetramethylchroman-2-carboxylic acid-Trolox- (Sigma-Aldrich); 50 or $100 \mu \mathrm{M}$ of $\mathrm{H}_{2} \mathrm{O}_{2}$ (Sigma-Aldrich); and MQ water as control, for five days at $23^{\circ} \mathrm{C}$. On the sixth day the explants were transferred to the germination table, which provides moist from flowing tap water, and grown at $23^{\circ} \mathrm{C}$. The $\mathrm{H}_{2} \mathrm{O}_{2}$ concentration levels were chosen based on similar studies in Arabidopsis (Tsukagoshi et al. 2010; Yu et al. 2016) and the concentration of Trolox was based on a similar study in tulip (van Rossum 1997).

\section{RNAseq}

Total RNA was isolated using the hot borate protocol (Wan and Wilkins 1994) with some modifications as described by Maia, 2011 (Maia et al. 2011). For Good-Basal (GB) explant material, RNA was isolated from all time points $(0,1,3,5,7$ and 9 DAC), for Good-Apical (GA) and Poor-Basal (PB), time point 5 was left out, and for Poor-Apical (PA), time points 5 and 9 were omitted to limit the total number of samples. These abbreviations are explained in Figure 1. RNA quality and quantity was measured by agarose gel electrophoresis and Qubit fluorimeter (Qubit v.2.0, Thermo Fisher Scientific), respectively.

The cDNA libraries were prepared with the TrueSeq sample preparation kit and the Ribo-Zero plant kit (Illumina, The Netherlands). The quality of the libraries was examined with the Bioanalyzer 2100 DNA 1000 chip (Agilent Technologies, United States). The samples were sequenced on a HiSeq2000 platform (100 bp paired-end; stranded sequence reads) and the quality of the reads was assessed with FastQC (http://www.bioinformatics.babraham.ac.uk/ projects/fastqc/). The reads were trimmed with Trimmomatic v.0.32 (Bolger et al. 2014a) and 
assembled de novo using Trinity v.2.0.6 (Haas et al. 2013a). Gene quantification was estimated by mapping the reads to the transcriptome using Kallisto v.0.42.1 (Bray et al. 2016). The estimated counts were normalized using the TMM method of the EdgeR package v.3.16.5 (Robinson et al. 2009) and the Edger Exact Test was used to determine the differentially expressed genes. The transcripts were annotated with both the best Arabidopsis and rice (Oryza sativa) BLAST hits, with a cut-off $<1 e^{-5}$.

Principal Component Analysis (PCA) was done with the GeneMaths software v.2.1 (Applied Maths BVBA, Sint-Martens-Latem, Belgium). The loadings of the second principal component were used to determine the Gene Ontology $(G O)$ over-representation of biological processes. GO enrichment analysis of differentially expressed genes (DEG) between 0-1 against 3-9 DAC was performed with the plugin BiNGO (Maere et al. 2005) for Cytoscape v.3.4.0 (Smoot et al. 2011 ), using the hypergeometric probability test and the Benjamini \& Hochberg false discovery rate (FDR) correction at a cut-off of 0.001 . The online tool ReviGO (Supek et al. 2011) was applied to remove the redundant $G O$ terms, using a cut-off of $<0.1$. GO enrichment analysis was also done for DEG between ODAC and all subsequent time points, respectively, for every explant type and using the same parameters as just described. In both analysis the corrected P-value was transformed into $\log _{10}$ (corrected $p$-value) for easier visual comparison using a heat map.

Self-Organizing Maps (SOM) were calculated for GB explant transcripts having expression $>0.5 \mathrm{CPM}$ in at least one time point. This reduces the chance of clustering based on absence of very low expression. The analysis was done with GeneMaths software v.2.1 (Applied Maths BVBA, Sint-Martens-Latem, Belgium) using a $3 \times 3$ grid and Pearson correlation.

\section{Scanning Electron Microscopy imaging}

Scanning electron microscopy (SEM) was done following the methodology described in Rossmann et al., 2015 (Rossmann et al. 2015). In short, fresh explants were frozen in liquid nitrogen, transferred to an Emitech K1250X (Emitech, http://www.quorumtech.com/home) for sublimation and coated with gold palladium before imaging. Imaging was done on a Supra 40 VP with a GEMINI column (Zeiss, http://www.zeiss.com/microscopy/en_de/home.html) at the Max Planck Institute in Cologne, Germany. All images were obtained and processed with the SmartSEN software.

\section{Edu staining and imaging}

At different time points after start of the culture (DAC), explants were transferred to in vitro

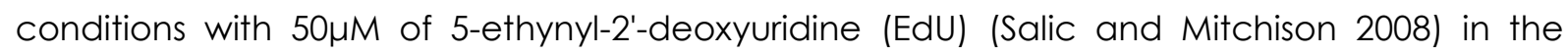
medium and cultured overnight at $23^{\circ} \mathrm{C}$. The explants were freshly sectioned with a Reichert Austria sliding microtome at 300 micrometres per section. The slices were treated with the ClickiT EdU Alexa Fluor 647 Imaging kit (Invitrogen) according to the manufacturer instructions and 
counterstained with $0.2 \mathrm{\mu g} / \mathrm{ml}$ DAPI. Imaging was done with a Zeiss LSM710 confocal microscope and Zen 2012 SP1 software v.8.1. Overlapping images were stitched together with Adobe Photoshop software (http://www.adobe.com) and the remaining empty areas were filled with grey pixels to generate rectangular panels.

\section{Hormonal measurements}

For cytokinin determination, $10 \mathrm{mg}$ of dry weight (DW) material was homogenized under liquid nitrogen, extracted in modified Bieleski buffer (methanol/water/formic acid, 15/4/1, v/v/v) (Novak et al. 2008), and then purified using MCX column (30 mg of C18/SCX combined sorbent with cation-exchange properties, Waters) (Antoniadi et al. 2015). Analytes were eluted by twostep elution using a $0.35 \mathrm{M} \mathrm{NH} 4 \mathrm{OH}$ aqueous solution and $0.35 \mathrm{M} \mathrm{NH} 4 \mathrm{OH}$ in $60 \%(\mathrm{~V} / \mathrm{v}) \mathrm{MeOH}$ solution. For auxins determination, $5 \mathrm{mg}$ DW material was homogenized under liquid nitrogen, extracted in $50 \mathrm{mM}$ Na-phosphate buffer, $\mathrm{pH} 7.0$ and then purified using HLB column $(1 \mathrm{ml} / 30$ mg, Waters) (Novak et al. 2012). Analytes were eluted using $80 \% \mathrm{MeOH}(\mathrm{v} / \mathrm{v})$. The levels of targeted compounds were determined using ultra high performance liquid chromatographyelectrospray tandem mass spectrometry (UHPLC-MS/MS) with stable isotope-labelled internal standards as reference ((Svacinova et al. 2012) for cytokinins and (Novak et al. 2012) for auxins. 


\section{Supplemental material}

Supplemental Table 1. Differentially expressed transcripts under the GO category "response to chemical stimulus" (http://www.wageningenseedlab.nl/thesis/nmorenopachon/SI/chapter5/)

Supplemental Table 2. Differentially expressed transcripts under GO categories related to cell proliferation. (http://www.wageningenseedlab.nl/thesis/nmoreno-pachon/Sl/chapter5/)

Supplemental Table 3. Differentially expressed transcripts under GO categories "organ development", "embryonic development" and "post-embryonic development". (http://www.wageningenseedlab.nl/thesis/nmoreno-pachon/SI/chapter5/)

Supplemental Table 4. Root identity, shoot identity and root-callus-leaf ( $R C L$ ) regeneration competence markers found in the lily time-series transcriptome. (http://www.wageningenseedlab.nl/thesis/nmoreno-pachon/SI/chapter5/)

Supplemental Figure 1. Protein sequence alignment of TgWOX13, Arabidopsis and rice WOX proteins. (http://www.wageningenseedlab.nl/thesis/nmoreno-pachon/SI/chapter5/ 


\section{CHAPTER 6}

\section{Histological origin of regenerated bulblets in lily bulb scales}

Natalia M. Moreno-Pachon; Annemarieke Borst; Marta Cubria-Radio; Richard G.H. Immink 


\begin{abstract}
Lily (Lilium sp) bulb scales have the remarkable ability to regenerate ectopic shoot apical meristems (bulblets) once the scales are detached from the mother bulb. Regardless of differences in regeneration potential between lily varieties, bulblets recurrently regenerate at the proximal-adaxial side of the scale, right on top of the excision line. Several studies point at auxin as one of the main initial factors required for plant regeneration and the same has been suggested for Lilium. However, there is limited information about the underlying mechanisms driving the efficient regeneration of de novo bulblets in lily scales. The aim of this study was to investigate the possible cellular and physiological factors that grant lily bulb scales their competence to regenerate. We propose that the excision wound disrupts the polar auxin transport, creates an auxin maximum at the excision line and causes a gradient of cell divisions favouring the adaxial parenchyma tissue. The direction of this cell division gradient depends on the regeneration competence of the tissue and the interaction with the epidermis. The adaxial parenchyma tissue seems to be more competent, partially due to the higher number of secondary veins and larger cell population than the abaxial parenchyma region because cells are smaller in the adaxial region. Moreover, the epidermis seems to cross-talk with the parenchyma cells to direct initial cell divisions and the position at which the ectopic meristems will occur. A better understanding of the composition and physiology of the epidermis in lily bulb scales could shed more light on the proposed importance of the epidermis and to elucidate the remarkable regeneration potential of Lilium $s p$.
\end{abstract}

Key words: Adaxial, abaxial, epidermis, auxin polar transport. 


\section{Introduction}

Lilies are monocotyledonous ornamental flowers with an extraordinary regeneration capacity. Lilies can propagate vegetatively, both by natural and artificial methods, by regenerating ectopic shoot meristems that will quickly produce scales, resembling a shoot bud (Bach and Sochacki 2012). Such ectopic shoot buds are called bulbils when located above ground and bulblets when regenerated below ground (Suh and Roh 2014). Natural regeneration methods include the formation of bulblets in the axil of the leaves, adventitious formation of bulblets along the proximal side of the stem and adventitious formation from the roots of the bulb. The most common commercial propagation method in lily is called "scaling". Scaling consists of detaching the scales from the bulb, chopping the scales in pieces and culturing these explants in a moist and warm environment to obtain bulblets (Bach and Sochacki 2012). The number and size of regenerated bulblets as well as the time required to regenerate them depends on the genetic background of the bulb and on the position within the bulb scale from which the explant was taken (chapter 5).

Lily bulb scales are modified leaves that serve as an underground storage organ (Le Nard and de Hertogh 1993). Interestingly, scaling in lily works for bulb scales, but it does not work for true leaves. True lily leaves require the addition of exogenous hormones and other components in the medium to be triggered for regeneration (Yin et al. 2013). Natural formation of ectopic meristems in leaves has been reported in some dicot species, such as tomato and begonia. In tomato, ectopic meristems can arise from a delimited (distal leaflet boundary) area within the base of the leaflet (Rossmann et al. 2015). This area is characterized by smaller epidermal cells and a lower density of trichomes and stomata, suggesting a lower level of differentiation and the possible pre-existence of a population of pluripotent cells, from which the ectopic meristems will initiate. On the other hand, in begonia, regenerated plantlets seem to arise de novo from the main vasculature of the leaf and leaf petiole once a leaf explant is excised and cultured in moist (Cassells and Morrish 1985).

The subsequent developmental stages of lily bulblet regeneration in the scaling approach have been described very well by Marinangeli et al (2003). The researchers reported that once the excised explant recovers from the wounding, the ectopic meristems initiate at the proximaladaxial side of the explant. They did not report finding a population of less differentiated cells in the area where the meristems initiate, although they suggested a tight link between the veins of the scale and the origin of such ectopic meristems (Marinangeli et al. 2003).

Meristem regeneration originating from the vasculature has been demonstrated in the root of the model species Arabidopsis (Atta et al. 2009; Sugimoto et al. 2010; Marhavý et al. 2012). Lateral roots emerge from the pericycle cells, which are pluripotent cells surrounding the vasculature of the main root (Dubrovsky and Rost 2001). Meristem regeneration via callus 
formation has also been documented in pericycle-like cells surrounding the vasculature of aerial organs (Sugimoto et al. 2010). Moreover, shoot regeneration from pericycle cells can occur directly without an intermediate step of callus formation (Atta et al. 2009). Independent of the exact regeneration route, pericycle cells require an auxin maximum to trigger the initial cell divisions that will ultimately give rise to the new meristems (Dubrovsky et al. 2008).

Auxin polar redistribution upon lily scale excision, as one of the first events required for bulblet regeneration, has been suggested already in the 80s of last century (Van Aartrijk and BlomBarnhoorn 1981). By adding an auxin polar transport inhibitor (TIBA), it was found that bulblets do not longer regenerate exclusively at the proximal side of the scale and elevated concentrations of TIBA inhibited bulblet regeneration completely (van Aartrijk and BlomBarnhoorn 1983). At the same time, the number of regenerated bulblets can be increased by adding low levels of synthetic auxin (van Aartrijk and Blom-Barnhoorn 1983). This increase is very likely caused by the accumulation of synthetic auxin at the proximal-adaxial region of the explant and the creation of more auxin maxima points from where the meristems can arise.

To date there is no clear evidence for the exact histological origin of bulblets regenerated from bulb scales. Although lily bulb scales constitute a good model system to study de novo regeneration in non-model monocot species due to their efficient regeneration, other constraints, such as the low transformation rate and lack of molecular tools, make it challenging to work with this species and system. Nevertheless, elucidating which characteristics confer lily scales their high regeneration potential could aid in understanding the recalcitrance in regeneration of other related and economically important species, such as tulip. In this study we aimed to investigate the possible cellular and physiological factors that grant lily bulb scales their competence to regenerate. For this purpose we focussed on the regeneration events consistently observed at the proximal-adaxial side of the bulb scale explant.

\section{Results}

\section{Investigation of proximal-distal regeneration differences}

To explore what mechanisms could control the recurrent proximal-adaxial regeneration in lily bulb scales, we performed two initial pragmatic experiments. First, since in our study system the explants were always cultured up-right with their proximal excision surface resting on the base of the moist chamber, we wondered whether gravity played a role in redistribution and creation of an auxin maximum at the base of the explant once it is excised from the bulb.

To test this idea we cultured explants upside down, in a way that the original proximal region became the new distal region (Fig. 1A). We expected to promote higher regeneration at the original distal side of the explant, which has now become the proximal side. Nevertheless, most of the bulblets regenerated at the new distal side (Fig. 1B-D) although some regeneration 
occurred also at the new proximal side (Fig. 1B,D). This observation suggests that there must be a mechanism to favour regeneration at the original proximal side of the explants such as remembering their original position. However, the regeneration percentage at the original proximal side reduced to half when cultured upside down (Fig. 1B), indicating that gravity can affect polar regeneration.

A

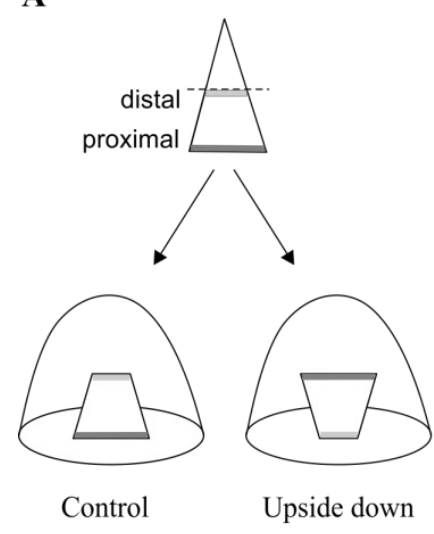

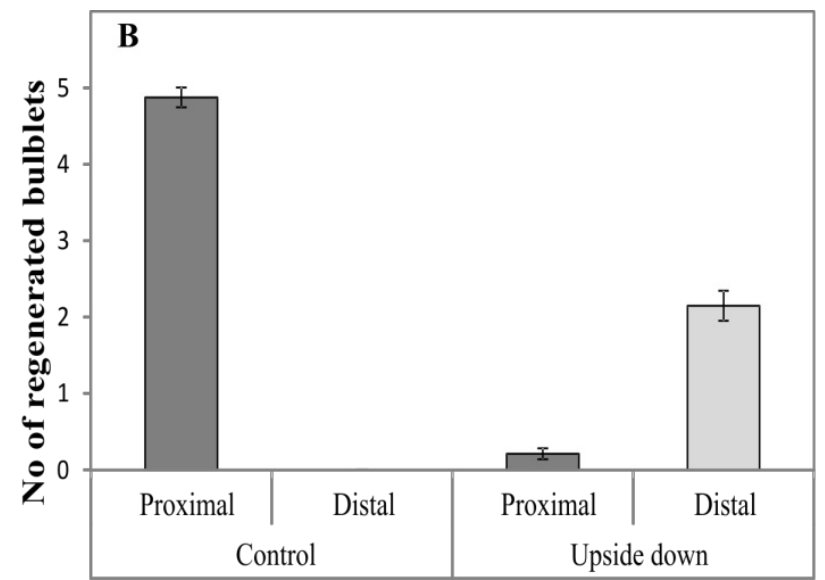

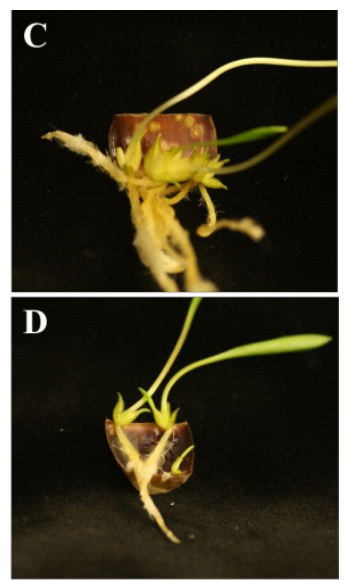

Figure 1. Influence of gravity during bulblet regeneration. A. Lily bulb explants were dissected from the scale in a way that a proximal and a distal excision were made. The explants were cultured upright with their proximal region in contact with the base of the moist chamber, or upside down with their distal region in contact with the base of the moist chamber. B. When explants are cultured upside down, the number of regenerated bulblets at the original proximal region diminished to half compared to when they are cultured with their proximal side at the base. Moreover, gravity can activate bulblet regeneration at the original distal area when the explant is cultured upside down. C. An average of four bulblets regenerates at the distal region of the explants cultured with their proximal side down. D. An average of two bulblets regenerate at the original proximal region when cultured upside down, and much less often they can also regenerate a small bulblet at the original distal region.

\section{Adaxial versus abaxial regeneration capacity}

Next, we wondered whether removing tissue layers at the proximal-adaxial region of the scale were normally regeneration occurs (Van Aartrijk and Blom-Barnhoorn 1981, 1984) would inhibit bulblet regeneration. To explore this possibility, the adaxial epidermis and half of the parenchymatic region was removed from the base to the mid height of the scale. As a consequence, bulblet regeneration was hindered at the remaining basal area while promoted towards the apical region, right above the excision line.

Interestingly, the few regeneration events observed at the basal area were located at the abaxial side of the explant. The results indicate that although the basal region has a higher regeneration competence over the apical region, this basipetal regeneration capacity is disrupted when the adaxial region is excised. Therefore, the adaxial region of the bulb scale must play an important role in promoting bulblet regeneration once the explant is excised or 
wounded. Another possibility is that the adaxial parenchyma tissue is more competent than the abaxial region.

A

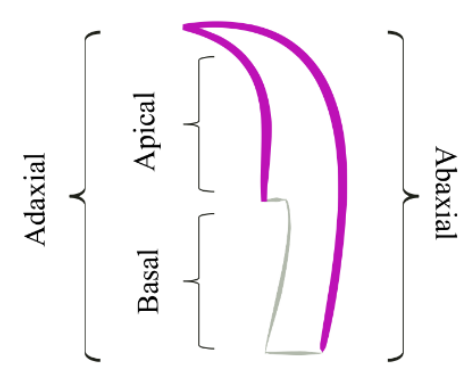

B

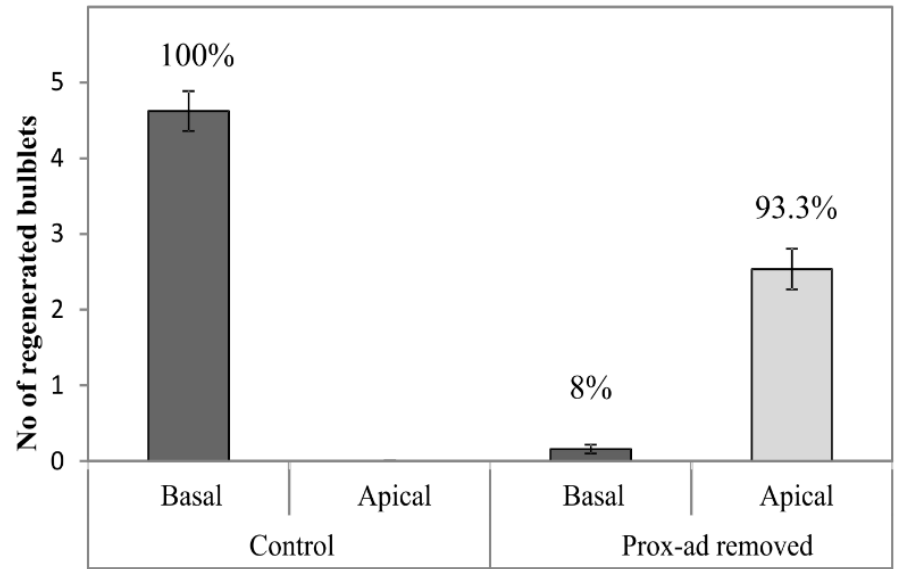

Figure 2. Bulblet regeneration in explants with their proximal-adaxial side removed. A. The adaxial epidermis and half of the adaxial parenchyma tissue was removed with a surgical blade. Explants were cultured in vitro in $1 / 2$ MS agar to prevent fungal infection on the extra wounded region. The epidermis is coloured in purple. B. Regeneration was mainly observed at the base of the apicaladaxial area (93.3\%), while a few regeneration events occurred at the basal area (8\%), but all at the abaxial side of the explant.

To explore the possible factors that favour bulblet regeneration at the basal and adaxial region we first examined the basic histological composition of the bulb scale. From outer to inner tissues the scale is composed of an epidermal layer, the parenchyma tissue and the vasculature (Fig. 3A). We did not observe differences in overall cell morphology between adaxial and abaxial epidermal and parenchymatic cells. Both adaxial and abaxial epidermal layers contained thin but elongated cells with no detectable starch granules. Also, the parenchyma cells in both regions looked similar and were large and rounded, containing multiple starch granules (Figs. 3A-C). Lugol staining was used to check whether there might be differences in the amount of starch granules throughout the explants; however, no differences in starch granule distribution could be identified (Fig. 3D).

The vascular tissue is located in the innermost area of the scale, surrounded by medium sized cells which lack the starch granules, typical of the parenchymatic cells (Figs. 3A and 3D). As a typical monocot leaf, the main vasculature of lily bulb scales is arranged in parallel to the length of the scale. On average, five main vascular bundles per scale could be detected in the apex of the scale and a few extra are found towards the base (Fig. 3A). Besides those main vascular bundles, the base of the scale contains tiny secondary vascular bundles which branch from the main vasculature, and they were mostly observed at the adaxial side (Fig. 3E-H). 
To investigate what seems to make the adaxial region more competent than the abaxial region we looked at the differences in both tissues. Light microscopy did not reveal apparent differences in e.g. cell shape or cellular starch content, other than a higher frequency of tiny secondary vascular bundles at the adaxial side (Fig. 3). However, when looking at the nuclei of the parenchyma cells it was evident that both basal and apical explants had on average 1.5 times more parenchyma cell nuclei at the adaxial region than at the abaxial region (Fig. $4 \mathrm{~A}-\mathrm{C})$. This implies that the abaxial parenchyma cells are larger than the adaxial parenchyma cells, resulting in less cells per given area.
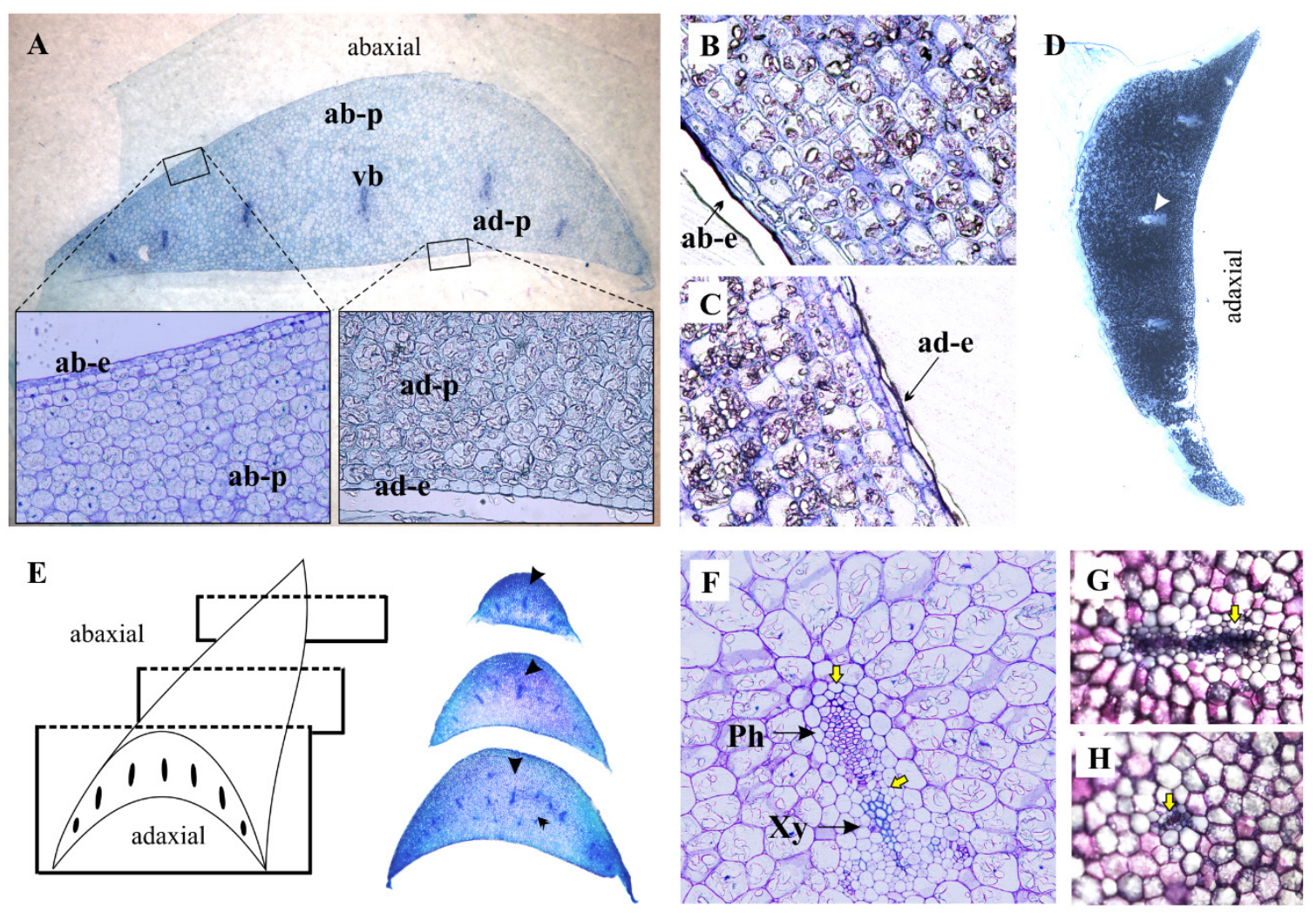

Figure 3. Histological description of lily bulb scales. A. Cross section of a bulb scale reveals similar composition and structure of the adaxial and abaxial regions of the scale. From outer to inner the scale is composed of the epidermis, followed by the parenchyma and the vascular tissue in the most inner area. Parenchyma cells are filled with starch granules, while epidermal cells did not stain and hence, contain no or little starch. ad-p: adaxial parenchyma. ab-p: abaxial parenchyma. vb: vascular bundle. B. Longitudinal cross section of the abaxial region. C. Longitudinal cross section of the adaxial region. D. Lugol staining reveals an even distribution of starch granules in the parenchyma tissue of the scale. White arrow points at a main vascular bundle, which is not stained because of the lack of starch within its cells. E. The vasculature of lily bulb scales is arranged in parallel and at some point they join before reaching the tip of the scale. Three main vascular bundles are observed at the apex of the scale and seven at the mid height and base. The base however contains more secondary vascular bundles than the mid and apex of the scale. Big arrow heads point at a main vascular bundle, small arrow heads at small secondary vascular bundles. F. The main vascular bundle contains the xylem (XY), characterized by thickened cell walls, and is located towards the adaxial side of the scale, while the phloem (Ph) faces the abaxial side of the scale. Yellow arrows point at starch-less cells that surround the vascular bundles. G. Main vascular bundle contains more starch-less cells than a secondary vascular bundle (see $\mathrm{H}$ ). 
To understand how this higher number of smaller adaxial parenchymatic cells could contribute to bulblet regeneration, we examined the proliferation rate of both regions in a time series. We found that within five days after culture (5DAC) the number of adaxial parenchymatic cells increased almost 5-fold, while only 2 -fold for the abaxial parenchymatic cells (Fig. 4D). Although this shows a perfect correlation of the parenchyma cell size differences between the adaxial and abaxial regions with propagation potential, the differences might not be caused by the size difference of the cells, but by differences in propagation stimulating signals at the adaxial and abaxial side of the explant. Moreover, a detailed look at the proximal-adaxial side of the explant at an early stage of meristem formation, showed that the meristematic domes arise by periclinal and anticlinal cell division of the parenchyma tissue, while the epidermal cells didn't show any sign of periclinal division activity, retaining the epidermis as a single cell layer (Fig. 4E-H).
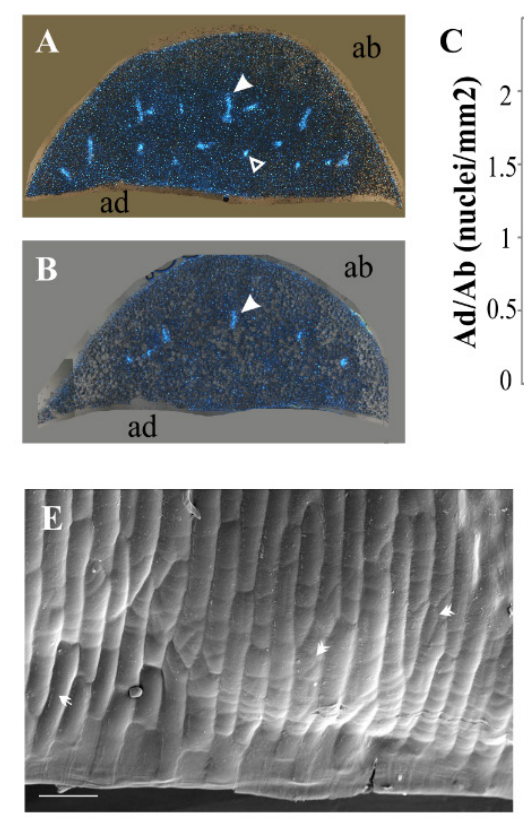
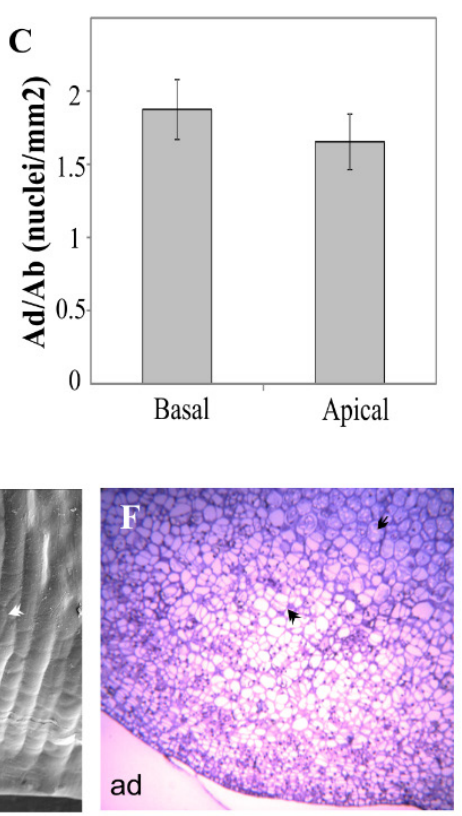
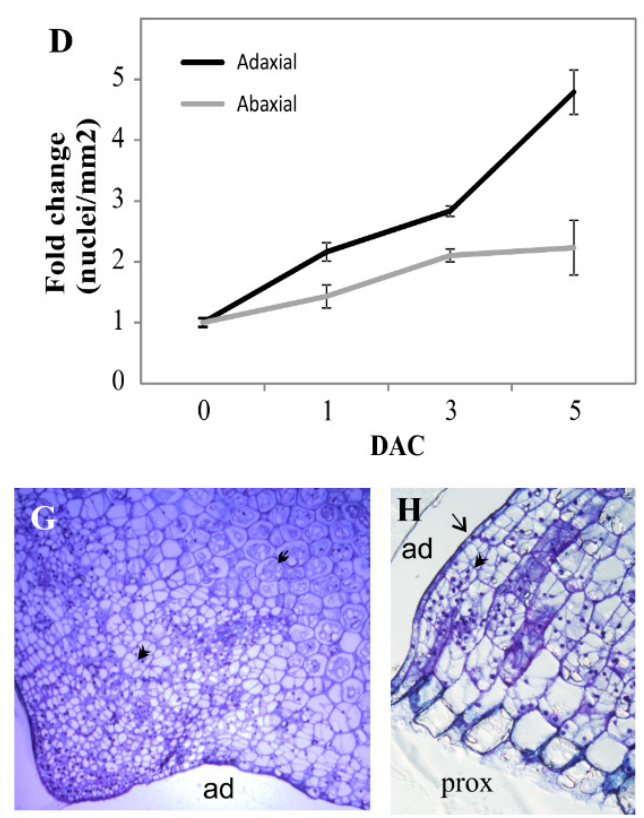

Figure 4. Influence of initial parenchyma cell number on bulblet regeneration and origin of bulblet regeneration. A. Cross section of a basal explant stained with DAPI. Big arrow head indicates a main vascular bundle, open arrow head a secondary vascular bundle. B. Cross section of an apical explant stained with DAPI. C. Basal and apical explants stained with DAPI reveal on average double the amount of parenchyma cells in the adaxial than in the abaxial area. D. Cell proliferation at the fifth day of culture (5DAC) increases five-fold in the adaxial area, while only two fold in the abaxial area. E. Scanning Electron Microscopy (SEM) of the proximal-adaxial side of an explant where a meristematic dome is forming. The typical long epidermal cells (white arrow) divide at this later dome stage of bulblet formation, resulting in smaller and irregular shaped epidermal cells (double arrow head). Bar: $100 \mu \mathrm{M}$. Cross (F-G) and longitudinal (H) section of the initial stage of the meristematic dome formation. The dome protrudes by periclinal and anticlinal cell division of the parenchyma cells. Single arrow in F-H points at the non-dividing parenchyma cell, double arrow head at the dividing parenchyma cells and arrow at the epidermal cells not showing periclinal cell proliferation in this initial stage of dome formation in the regeneration process. 


\section{A role for the epidermal cell layers?}

Since upon removal of the proximal-axial tissues, including the epidermis, regeneration occurred at the base of the more distal adaxial region and the abaxial side of the explant, where the epidermis was still intact (Fig. 2), we wondered if the epidermis might be crucial in

A
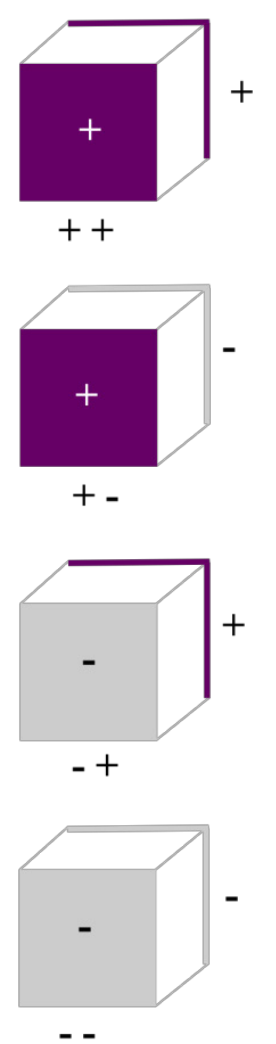
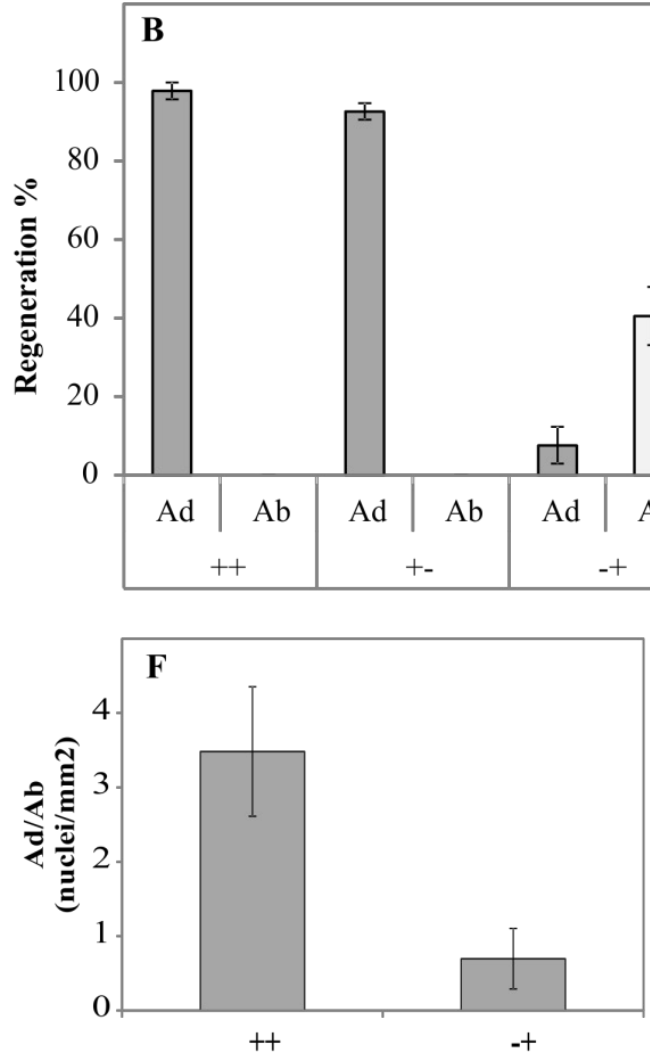
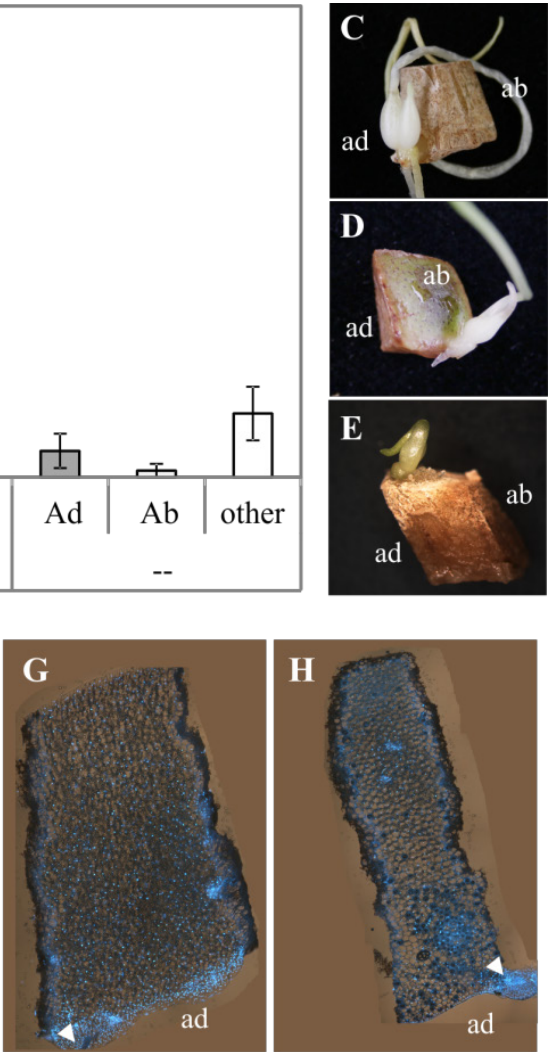

Figure 5. Influence of the epidermis on bulblet regeneration. A. Explants of approximately $0.4 \times 0.4 \times$ $0.4 \mathrm{~cm}$ were excised from the scale and their adaxial and abaxial epidermis were left intact $(++)$, removed at the abaxial $(+-)$, at the adaxial $(-+)$ or at both sides $(--)$ and cultured in vitro. B. Bulblet regeneration percentage at the adaxial (ad) and abaxial (ab) region was assessed in the four types of explants. Results indicate that when the adaxial epidermis is removed general regeneration capacity diminishes and is favoured at the abaxial side. Removing both epidermises is detrimental for bulblet regeneration and it also disrupts regeneration polarity for the rare occurring regeneration events. C. Bulblet regeneration at the adaxial side in a "- +" explant. D. Bulblet regeneration at the abaxial side in a "- +" explant. E. Bulblet regeneration in a "- -" explant. Note that the bulblet regenerated on top of the explant. F. Ratio of adaxial/abaxial number of nuclei per $\mathrm{mm}^{2}$ in explants with their adaxial epidermis removed "- +" when a regeneration took place at the abaxial side. The ratio indicates that cell proliferation was favoured at the abaxial side, in contrast to the control situation "+ +". G. Nuclei visualization by DAPI staining in "+ +" explants with a regenerated bulblet at the adaxial side. H. Nuclei visualization by DAPI staining in "- +" explants with a regenerated bulblet at the abaxial side. White arrow head indicates the base of the regenerated bulblet.

constraining bulblet regeneration. To test this hypothesis, we cultured explants of approximate $0.4 \times 0.4 \times 0.4 \mathrm{~cm}$ with their adaxial, abaxial, or both outer layers removed, and assessed their regeneration capacity (Fig. 5A). We found that the epidermis does contribute to the 


\section{Chapter 6}

regeneration competence because explants without any of these layers hardly regenerated (Fig. 5B). Moreover, the few regeneration events in this type of explants are located at random and no longer at the proximal-adaxial side (Fig. 5E). Also, it seems that adaxial epidermal layer exerts a higher influence in the regeneration capacity because when absent, regeneration diminishes dramatically and switches to the abaxial side (Figs. 5B and 5D), while there is no significant effect when the abaxial epidermis is absent (Figs. 5B and 5C).

This result suggests the existence of a signalling component, such as a hormone, protein or metabolite in the epidermal layer that confers enhanced regeneration capacity. To proof this idea we cultured epidermis-less explants with or without adding the ground material of the removed epidermis to the culture medium. Unfortunately, no significant rescue of regeneration capacity could be observed (Fig. 6).
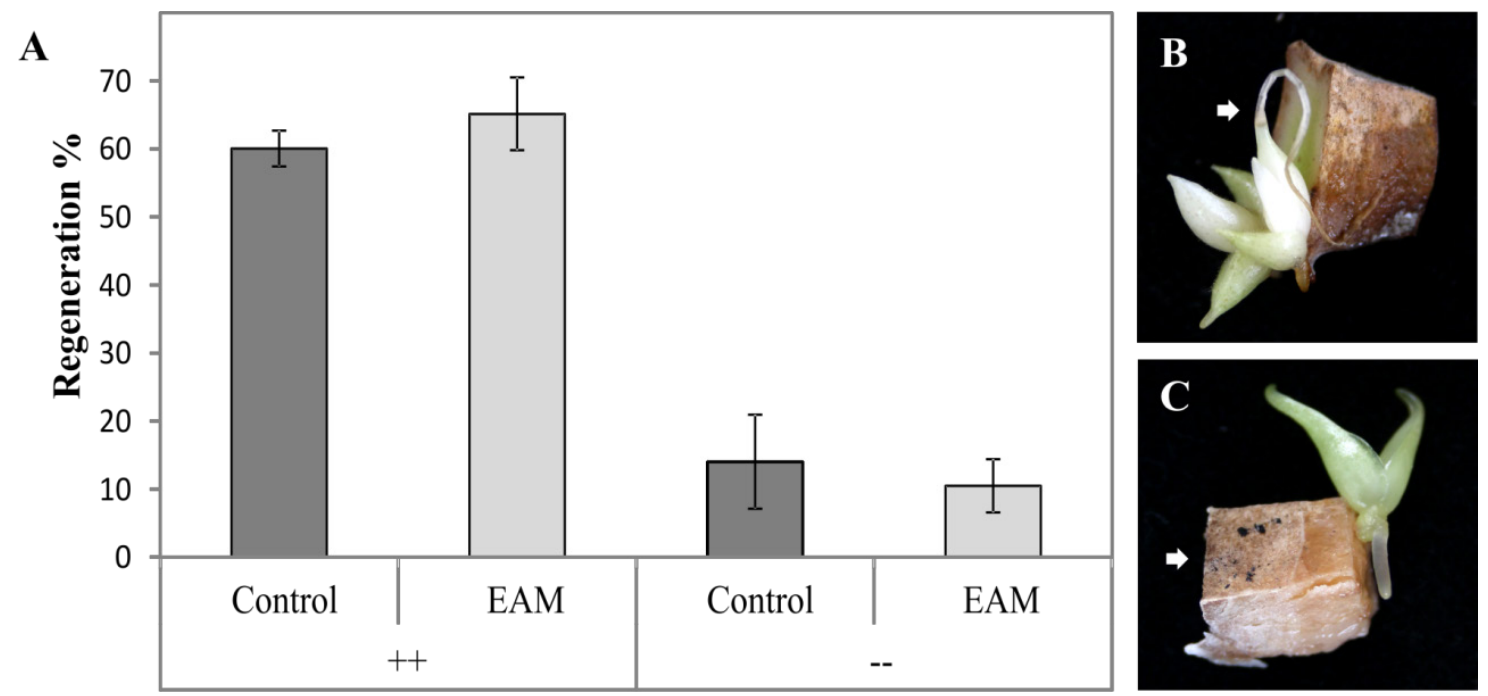

Figure 6. Adding the epidermis to the growth medium did not rescue the poor regeneration capacity of epidermis-less explants. A. Regeneration percentage of explants with $(++)$ or without (-) epidermis, grown in standard medium (Control) or with freeze dried and ground epidermis added to the medium (EAM). B. Regeneration of ++ explants grown at controlled conditions. C. Regeneration of - - explants grown at EAM, the bulblet rose at the distal abaxial side of the explant. White arrows indicate the adaxial side of the explant.

\section{A role for the vasculature in bulblet regeneration?}

The test the previously proposed link between the vascular bundles and bulblet regeneration (Marinangeli et al. 2003), we cultured explants of approximately $0.4 \times 0.4 \times 0.4 \mathrm{~cm}$ with and without a main vascular bundle (Figure 7A) and assessed their regeneration capacity. We reasoned that if the vasculature is playing a positive role during bulblet regeneration, explants holding a main vascular bundle would show a faster and higher percentage of regeneration than explants without a primary vascular bundle. Nevertheless, although explants without main 
vascular bundle seemed initially to reach a higher percentage of regeneration, both type of explants reached $50 \%$ of regeneration at 15 DAC. After this time, explants with a main vascular bundle reached $100 \%$ regeneration significantly faster than explants without vasculature (Figure 7B-C), but both reached a similar number of regeneration events (1 bulblet/explant). Moreover, no cellular protrusions were observed from the vascular bundle towards the adaxial side (Figure 7D).
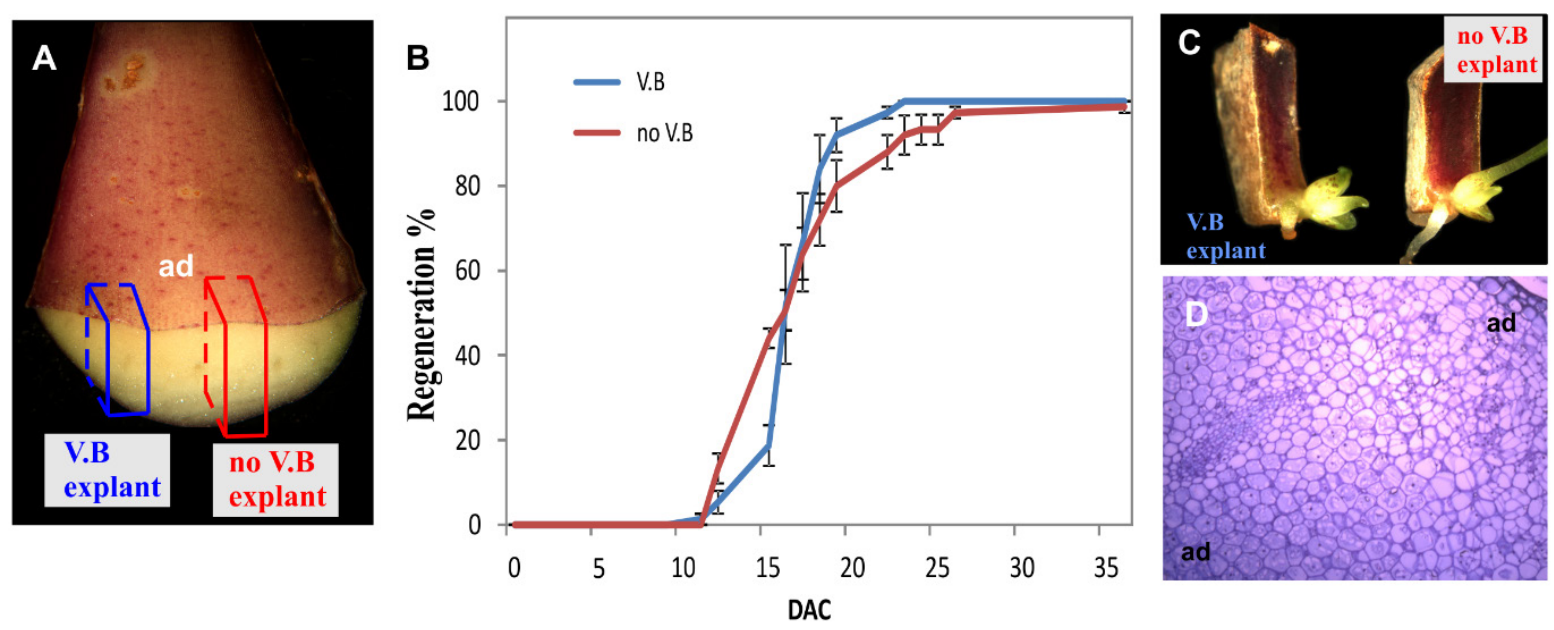

Figure 7. Influence of main the vascular bundles during bulblet regeneration. A. Small explants $(0.4 \times 0.4 \times 0.4 \mathrm{~cm})$ were excised from the scale holding or not holding a main vascular bundle. $B$. The regeneration percentage in both types of explants was very similar and both reached $50 \%$ of regeneration by 16DAC. C. Bulblet regeneration in explants holding a main vascular bundle or not. Both type of explants regenerated one bulblet per explant. D. Cross section of an explant with a vascular bundle at 10DAC. No meristem dome was observed yet but there are many cell divisions ongoing at the adaxial parenchyma tissue, and they do not seem to protrude from the vascular bundle.

\section{Discussion}

Lily is a species with an enormous regeneration efficiency and this regeneration capacity is of special interest because it appeared to be inherent to particular cells in e.g. bulb scales and occurring without the need for external hormonal treatments. Intrigued by this observation, we decided to study from which particular cells regeneration of new bulblets in scales is initiated and to explore which factors equip these cells and tissues with their high regeneration capacity. The fact that the explants require a transversal excision line to regenerate bulblets and that those bulblets arise at the base of the explant, on top of the excision line, suggests that a regeneration promoting substance accumulates at the excision line due to basi-petal (towards the root) longitudinal transport disruption. In many experimental systems (reviewed in (Ikeuchi et al. 2016)), including lily (Van Aartrijk and Blom-Barnhoorn 1981), auxin has proven to be a regeneration triggering hormone and hence, a good candidate for the basi-petal 
transported regeneration signal in lily scale explants. In leaves, auxin is produced and translocated to the root passively through the phloem (De Schepper et al. 2013) or actively through epidermal and endodermal cell layers of the leaf and stem (Morelli and Ruberti 2002; Blilou et al. 2005). This transport is mediated by transmembrane proteins such as the auxin influx (AUXI/LIKE AUX1 (AUX1/LAX) family of proteins and the efflux PIN FORMED (PINs) proteins (Petrasek et al. 2006; Vieten et al. 2007). Since bulb scales are modified leaves, its auxin transport pattern presumably resembles that of the leaves.

\section{What favours bulblet regeneration at the proximal side of the explant?}

We showed that gravity has an effect on the position of regeneration and that regeneration at the distal side can be achieved, although at low rate, when the explant is cultured upside down. This shows some remembrance of the original orientation of the explant and that probably, for at least a while, auxin is transported towards the original base. However, the recurrent regeneration at the proximal side seems to be controlled by gravity and probably, a reorientation of the auxin transport direction.

In Arabidopsis, shoots and roots remember their orientation through the sedimentation of starch granules at the base of the columella cells in the root (Blancaflor et al. 1998) and endodermal cells in the shoot (Fukaki et al. 1998; Friml et al. 2002). When tropism changes, such starch granules redistribute at the new base of the cell, inducing a re-polarization of PIN3 proteins within 3 minutes, to create an asymmetrical auxin redistribution that helps the shoot or root reorientate (Friml 2003). So far PIN3 proteins have only been observed in endodermis and gravity sensing tissues (Friml et al. 2002).

It is possible that lily bulb scales possess a similar mechanism to remember their original orientation, so upon excision, polar auxin transport towards the original proximal excision line is activated to induce regeneration as a survival mechanism. Unfortunately, no PIN protein fluorescent marker lines are available in lily to prove this hypothesis. Endodermis cells in the shoot (stems and leaves) are located at the periphery of the vascular bundles. Nevertheless, we did not observe starch granules in those cells, but mainly in the parenchyma cells. Thus, if lily scales possess a starch-based mechanism to keep track of their orientation, it is more likely that such mechanism lays in the parenchyma cells. Given the enormous amount of starch granules within the parenchyma, it is tedious to image the cellular organelles by light microscopy but electron microscopy in scales with less starch e.g. young scales, could test the existence of this starch-based orientation mechanism.

The fact that apical explants regenerate less bulblets and at a slower pace than basal explants, although both should have the same mechanism to activate basi-petal polar transport upon wounding, might be due to differences in regeneration competence. But what causes those differences in competence level? We did not observe morphological differences between 
apical and basal tissues other than the number of vascular bundles. Basal explants have more main and secondary vascular bundles due to branching of the vasculature to cover the wider area at the base of the scale. If the pericycle-like cells that surround the vasculature were the source of regeneration, as has been reported for Arabidopsis (Sugimoto et al. 2010), having more veins would increase the regeneration capacity of the explant. Nevertheless, we did not observe differences in final regeneration percentage and only differences in regeneration speed in explants with or without a main vascular bundle. Moreover, we did not see any cellular protrusion coming from the vascular bundle towards the adaxial region, as happens when shoots regenerate from root pericycle cells (Atta et al. 2009).

Another explanation could be that having more vascular bundles per unit area can provide the explant with more vascular transported resources, such as hormones. As stated before, hormones can be passively transported via the phloem, but also actively throughout the plant via the endodermis and the epidermis (Morelli and Ruberti 2002; Blilou et al. 2005). It is possible that when we excised explants without a main vascular bundle, there were still secondary veins, reason why regeneration was not affected in such explants. If that was the case, it would mean that regardless the size of the vascular bundle, they promote regeneration by redistributing the necessary hormones upon wounding. To proof this idea further, explants without any vein should be tested, but unfortunately, in practice this is not an option because it is impossible to excise an explant of sufficient size without any vein tissues.

\section{What makes "adaxial" so special in lily bulb scales?}

Another recurrent event during lily bulblet regeneration is the adaxiality of regeneration. Besides finding more secondary veins in the adaxial area, we also found smaller and hence, more parenchyma cells. This adaxial parenchyma cells proliferated at a higher rate than the abaxial ones, creating a gradient of cell proliferation favouring the adaxial side of the scale. Thus, the combination of more veins and a bigger population of starting cells in the adaxial parenchyma tissue seems to grant more competence for bulblet regeneration.

We also showed that this gradient in cell division can be inverted, although at low frequency, when the adaxial epidermis of the explant is removed. In that case, regeneration arises at the abaxial side of the scale, where the abaxial epidermis is present. This indicates that both adaxial and abaxial regions in the scale are competent to regenerate bulblets, although at different level, and that the epidermis plays an important role during regeneration.

We hypothesise that the epidermis contains a hormone, hormone-like or peptide compound that is able to trigger regeneration in adjacent parenchyma cells, and that this trigger is probably produced at higher levels by the adaxial than the abaxial epidermis. Unfortunately, our attempt in a pilot experiment to activate adaxial regeneration by adding ground adaxial 
epidermal material to the medium did not result in the rescuing of regeneration in epidermisless explants, neither in enhanced regeneration in control explants. It might be that the epidermis dose was insufficient or that the triggering factor got degraded while processing the epidermis material. Nevertheless, since the few regeneration events in epidermis-less explants did not follow the normal basipetal polarity, it is possible that the epidermis has a role in positioning the future ectopic meristems. The fact that an intact epidermis is crucial for certain key processes in plant development, shoot growth and plant defence and that the epidermis is in constant cross talk with the surrounding tissues (Javelle et al. 2011), lead us to speculate that the epidermis plays an important role during lily bulblet regeneration.

\section{Conclusions}

Detailed morphological observations upon perturbation of the original 'scaling' protocol (Rees 1992) provided insight in the cellular and physiological origin and causes of regeneration capacity in lily. Our results and previous observations of regeneration capacity upon treatment of explants with the polar auxin transport inhibitor TIBA (van Aartrijk and Blom-Barnhoorn 1983), indirectly support the idea that wounding and auxin redistribution are tightly linked to promote bulblet regeneration in lily bulb scales. Based on the location of first cell divisions and the position of regeneration we propose that there must be cross talk between the wounded cells, the epidermis and the inner tissues which transport hormones.

The wound creates a disruption of polar auxin transport, auxin accumulates at the wounded area and it triggers cell proliferation and dedifferentiation which are required for bulblet regeneration (Chapter 5). The higher competence of the adaxial parenchyma tissue produces a gradient of cell proliferation towards the adaxial epidermis, which will result in the formation of ectopic meristems. The epidermis may play several putative roles during lily bublet regeneration. It may control part of the polar auxin transport, guide the new auxin flux upon auxin accumulation at the wounded area, provide an essential signal for regeneration (e.g. peptide) or a combination of the above options. Thus, a better understanding of the composition and physiology of the epidermis in lily bulb scales will shed more light on the elucidation of the remarkable regeneration potential of Lilium sp.

\section{Materials and Methods}

\section{Plant material and culturing conditions}

Mid-located lily bulb scales were detached from the mother bulb, rinsed with running water, dipped for 1 minute in $70 \%$ ethanol, then transferred to $2 \%$ sodium hypochlorite (V/V) for 20 minutes, and finally rinsed three times with sterile $M Q$ water for 1, 5 and 10 minutes, respectively. The scale explants were cultured either in moist chambers or in vitro. In the first case the explants 
were cultured on a germination table within moist chambers with flowing water at $23^{\circ} \mathrm{C}$ and a $12 / 12 \mathrm{hr}$ day/night light rhythm. When cultured in vitro, $1 / 2$ strength MS medium was used with $0.3 \%$ phytagel (W/V), and explants were cultured at $24^{\circ} \mathrm{C}$ and a $12 / 12 \mathrm{hr}$ day/night light rhythm.

\section{Culturing explants upside down}

Explants with a proximal and a distal excision line were cultured up-right either with their proximal excision line placed at the base of the moist chamber, or upside down, with their distal excision line placed at the base of the moist chamber. In the second case, the original proximal area became the new distal area and vice versa for the original distal area. The number of regenerated bulblets was assessed at 35 days after culture (35 DAC).

\section{Culturing of scales with their proximal-adaxial epidermis removed}

Entire bulb scales were used in this experiment. The proximal and adaxial epidermis and part of the adaxial parenchyma tissue was removed with a surgical blade. The mid height of the scale delimited the proximal from the distal region. The remaining basal area of the explant contained part of the adaxial parenchyma tissue, the main vascular bundles and the abaxial parenchyma and epidermis. The explants were cultured in vitro to reduce the chance of fungal infection at the wounded area. The number of regenerated bulblets was assessed at 45 DAC.

\section{Preparation and culturing of explants with or without a main vascular bundle}

Explants of approximately $0.4 \times 0.4 \times 0.4 \mathrm{~cm}$ were excised from the bulb scale at the region between two main vascular bundles, or having a vascular bundle in the middle. The first explants were considered as not having a main vascular bundle, although we cannot rule out the possibility that little secondary veins were present. Both explants with and without a main vascular bundle were cultured in vitro standing up-right and bulblet regeneration was assessed at 45 DAC.

\section{Culturing of explants with their adaxial, abaxial or both adaxial and abaxial epidermis removed}

Explants of approx. $0.4 \times 0.4 \times 0.4 \mathrm{~cm}$ were excised from the bulb scale and their adaxial, abaxial or both adaxial and abaxial epidermis was removed with a surgical blade. The explants were coded as "+ +" when no epidermis was removed; "+ -" when only the abaxial epidermis was removed; "- +" when only the adaxial epidermis was removed; and "- -" when both epidermal layers were removed. The explants were cultured in vitro standing up-right and bulblet regeneration was assessed at 45 DAC. 


\section{Culturing of explants with ground material from the adaxial epi- and endodermis added to the medium}

The adaxial outer layers of many sterile mid-located bulb scales were excised with a surgical blade, collected in liquid nitrogen and freeze dried overnight. The dried epidermis was ground into a powder and added to the $1 / 2$ strength MS medium in a $1.3 \%(\mathrm{~W} / \mathrm{V})$. As control the same $1 / 2$ strength MS medium was used, but without epidermis material added.

Explants of aprroximately $0.4 \times 0.4 \times 0.4 \mathrm{~cm}$ were excised from the bulb scale and both adaxial and abaxial epidermis were left intact or removed (+ + and - -, respectively). The explants were cultured in vitro (in medium either containing, or not containing, the ground epidermis) in 24well suspension culture plates (Greiner-Bio One) with $2 \mathrm{ml}$ medium per well. The explants with epidermis $(++)$ were cultured up-right on the medium. Explants without epidermis (- -) were submerged in the medium to avoid dehydration. Bulblet regeneration was assessed at 45 DAC.

\section{Toluidine Blue and Lugol's iodine staining and microscopic imaging}

Explants were collected and immediately vacuum infiltrated in $4 \%$ formaldehyde for 30 minutes and transferred to fresh $4 \%$ formaldehyde overnight. Next day, the explants were dehydrated in an ethanol series (10,30, $50,70,90,96,100,100 \%$ ) and infiltrated with Technovit 7100 (including hardener 1) in three steps (Hereaus Kulzer, Germany), followed by Technovit 7100 plus hardeners 1 and 2 reagents (Hereaus Kulzer, Germany). The explants were sectioned to a thickness of $20 \mu \mathrm{m}$ with a rotary microtome (Zeiss HM340E). Sections were stained in $0.05 \%$ tolvidine blue (Merck, Germany) or 0.33\% Lugol's iodine (Sigma-Aldrich) for two minutes, rinsed with water and dried on a warm plate at $42^{\circ} \mathrm{C}$. the sections were mounted and imaged with a Zeiss Axioskop microscope with DS-U2 camera and NIS-Elements imaging software (Nikon).

\section{DAPI staining and visualization}

Five representative explants per treatment were freshly sectioned with a Reichert Austria sliding microtome with a thickness of $300 \mu \mathrm{m}$. Three cross sections per explant were stained with 1.25 $\mathrm{ug} / \mathrm{ml}$ of DAPI. Imaging was done with a Zeiss Axioskop epifluorescent microscope with DS-U2 camera and NIS-Elements imaging software (Nikon). The overlapping images of one cross section were stitched together with Adobe Photoshop software (http://www.adobe.com) and the resulting empty area was filled with the picture background pixels to generate rectangular panels. Three random field areas without main or secondary vascular bundles were selected 
Histological origin of regenerated bulblets in lily

to count the number of nuclei per $\mathrm{mm} 2$. The number of nuclei was assessed with Image J software v.1.490 and the average of three field areas made one biological observation. 

CHAPTER 7

General discussion 


\section{A molecular approach to study vegetative propagation in non-model and bulbous species}

Model species are critical for a thorough understanding of the mechanisms supporting life in the different kingdoms. Plant model species have relatively low number of chromosomes, short life cycles, extensive genetic and "omics" datasets available, and are easily transformed. In that sense, model species can function as interactive encyclopaedias which cover several groups, as it is e.g. the case for Arabidopsis thaliana representing eudicots and Oryza sativa representing monocots. However, given the complexity of plant life and survival mechanisms in the plant kingdom, model species do not always cover all specific traits of certain taxonomical ranks.

Geophytes, such as lily and tulip, are taxonomically highly diverse (more than 800 different genera) (Benschop et al. 2010), they have complex and lengthy life cycles, large genomes, and diverse vegetative propagation strategies. Nevertheless, we prove in this thesis that model species are of great value to get a better understanding of regulatory pathways in bulbous plants, e.g. for gene annotations (chapter $\mathbf{3}$ ), identification of specific gene features (TgTBI in chapter 4; LOERFI15 and LOWOX-like13 in chapter 5), and design of targeted molecular approaches (chapter 4). However, model species would fail to recreate the complex plant architecture of tulip bulbs or the de novo regeneration capacity of lily bulb scales. Thus, definitely more research is needed to build comprehensive datasets of bulbous plants and of non-model species.

Several studies in the past century that aimed to improve the propagation capacity of tulip have adopted a classical physiological approach. Classical physiological studies must not be underestimated, because various important horticultural practices used to date come from such approach. One example is the development of "forcing" as a technique to shorten the tulip life cycle for cut flower production industry (Benschop et al. 2010). However, interpreting merely physiological assessments without a fundamental understanding of the processes and mechanisms behind, can be misleading and sometimes inaccurate. For example, different hormonal, chemical, and temperature treatments have been applied to bulbs of different species and at different stages of the growing cycle, or to explants grown in vitro, in order to influence the propagation capacity (summarized by (Okubo 2012; Bach and Sochacki 2012)). One example is that ethylene applied to tulip mother bulbs after anthesis can increase the number of daughter bulbs, but it also brings irreversible negative effects to other tissues. The authors proposed back then, in 1976, that DNA and RNA studies were necessary as a follow up to understand the true effect of ethylene on tulip morphogenesis (Klyne and Phan 1976). Nevertheless, despite the enormous flight molecular studies have taken in plant model species over the last decades, studies in bulbous species are rare. 
Genetic studies have broadened the knowledge of e.g. resistance traits in flower bulbs (BarbaGonzalez et al. 2005; Baldwin et al. 2014; van Tuyl et al. 2012), but their resolution is significantly affected by the high heterozygosity level (Benschop et al. 2010), the resolution of the physical map, and the quantitative variability of the trait. An attempt to find putative quantitative trait loci (QTL) for axillary bud outgrowth capacity in tulip through a genetic approach was undertaken at the start of this PhD study (data not included in this thesis), using a Tulipa gesneriana $\times$ T. cantata population (Tang et al. 2015). Unfortunately, the variation in number of daughter bulbs produced was limited and together with the relative small size of the population, this approach was not powerful enough to detect genomic loci influencing vegetative reproduction capacity. A way to solve this issue, is to generate different, welldesigned, and large segregating tulip populations. However, this is a very laborious and time consuming approach with the current breeding methods because of the lengthy life cycle of tulip. This highlights even more the necessity to invest in an alternative "omics" approach that would pave the road for future studies.

As stated in chapter 1 and $\mathbf{2}$ of this thesis, molecular approaches in bulbous plants bring a lot of challenges because of the huge size of their genomes and lack of a reference genome sequence from any bulbous plant species. Therefore, we chose to focus on the transcriptome of both tulip and lily to reduce the level of complexity; however not having a reference genome brought other challenges, such as the enormous number of in silico predicted gene isoforms, as can been seen from chapter 3 .

One of the biggest challenges when studying vegetative propagation in flower bulbs at the molecular level is the difficulty of functional analysis to prove predicted gene functions. First because of the lack of a rapid, stable and efficient transformation protocol that results in the formation of bulbs instead of shoots (Núñez de Cáceres et al. 2011); and second because model species do not form bulbs, so translational research based on model species is either not possible, or could be misleading. For example, bulbs are considered compressed plants where bulb scales are homologues to leaves (De Hertogh and Le Nard 1993b) and as such, knowledge on development of a model species may still be informative. However, the fact that natural and de novo bulblet regeneration in lily is only highly efficient in bulb scales and not in true leaves, is an indication that the high regeneration capacity of this species is inherent to specific and probably unique features of bulb scale tissue.

Due to the lack of efficient methods, no functional analysis of lily or tulip genes was carried out in this PhD project; however, it does not necessarily mean that it is not possible to prove the function of some of the identified and highlighted genes in the near future. Transient gene silencing in lily bulb scales could bring valuable understanding of the potential key regulators of de novo regeneration in this species. This functional analysis could be achieved using virusinduced gene silencing (VIGS) by vacuum infiltrating small bulb scale explants. This 
methodology has recently proven to work in the geophyte species Gladiolus hybridus, when used to silence ABSCISIC ACID INSENSITIVE 5 (GhABI5) in cormels (Wu et al. 2015), which are underground storage organs, representing structures homologous to daughter bulbs in tulip, or to bulblets in lily. Furthermore, VIGS was recently successfully applied in lily vegetative tissues and flowers (Tasaki et al. 2016), highlighting the potential of this methodology. Additional information about possibilities of this methodology will be discussed further on in this chapter.

\section{What can we learn from axillary bud outgrowth in tulip?}

In chapter 4 it was shown that although TgTBI expression correlated with the degree of axillary bud growth ('A' buds never ceased to grow and this correlated with their low expression of TgTB 1; whereas dormant ' $D$ ' buds showed higher relative expression of TgTB 1), downregulation of TgTB1 in dormant buds was not sufficient to sustain their growth. We also observed that the time of axillary bud initiation plays a fundamental role in determining the dormancy status of the buds. For example, the number of bulb scales might be linked to the time of axillary bud initiation. To understand better this axillary growth regulation, the plant architecture and developmental processes have to be taken into consideration. The first important aspect to consider is that three generations can be contained in one bulb: the mother bulb, its axillary buds, and the axillary buds of the axillary buds. In other words, one mature (flower bearing) mother bulb at planting time contains on average six daughter buds (axillary buds), which in turn will produce grand-daughter buds by the end of the growing season (Rees 1968). At the practical level, this means that any treatment given to the mother bulb, aiming to remove axillary bud dormancy, is also received by the daughter and grand-daughter buds and therefore, would very likely show its effects by the end of the following growing cycle.

The second aspect is that axillary meristems are formed from outside to inside in the bulbs and that in principle, these initiated buds will make one or two scales and then enter a dormant state (Chapter 4, (Rees 1966)). However, the buds that are initiated when the shoot apical meristem (SAM) of their mother has transitioned into the reproductive state ('A and B' buds in tulip), are able to skip this kind of dormancy (Chapter 4 and (Rees 1968)). This particular dormancy is known as paradormancy and imposed by apical dominance (Lang et al. 1987). Thus while 'A' buds are formed later but continue forming new scales during the growth cycle, ' $D$ ' buds remain with two or three scales only. It seems logical to think that the growth capacity of an axillary bud is linked to the number of scales and that in that sense, 'A' will always grow more than ' $D$ ' buds. However, if ' $D$ ' buds have the potential to re-initiate scale formation once TgTBI is levelled down after anthesis of the mother bulb, why does it not occur? Based on our results, it is tempting to propose that the SAM of ' $D$ ' buds loses responsiveness to re-initiate scale formation when subjected for too long to TgTB I. This responsiveness is very likely controlled by activating and repressing signals targeting the meristem. These signals could come from players 
of the TgTBI pathway, such as hormones; or could be independent, such as callose deposition in the plasmodesmata; or related to sink strength.

Therefore, since the meristematic activity of tulip axillary buds was not studied in this thesis, it is worth investigating whether the SAM of a dormant bud is really inactive after TgTB 1 expression is downregulated. Cyclins (CYC), HISTONE 4 (H4) and HISTONE $3(\mathrm{H} 3)$ would be good molecular markers to study meristem activity and competence (David Law and Suttle 2004; Gaudin et al. 2000). Moreover, the expression of other marker genes involved in axillary bud outgrowth or repression identified in model species or other geophytes could bring more insight into the regulatory mechanism in tulip. Examples of those genes are 9-CIS-EPOXICAROTENOID DIOXIGENASE 3 (NCED3), ABSCISIC ACID INSENSITIVE 5 (ABI5), and CAROTENOID CLEAVAGE DIOXYGENASE8 (CCD8), (Yao and Finlayson 2015; González-Grandío et al. 2017; GomezRoldan et al. 2008; Liang et al. 2010; Pasare et al. 2013; González-Grandío and Cubas 2014; Wu et al. 2015; Hartmann et al. 2011).

The complexity in the architecture of tulip bulbs is reflected in the differential outgrowth efficiency of axillary meristems and hence, propagation rate through axillary bud development. Researchers interested in increasing this rate could think of a way to increase the number of the innermost axillary buds, which are the ones that are the latest formed after the SAM has switched to reproductive development and that skip dormancy. Although it was not reported in chapter $\mathbf{4}$, the external scales had sometimes two axillary buds located in close vicinity. The frequency of this axillary bud duplication depended on the cultivar. But as being ' $D$ ' or ' $E$ ' type of buds, they fell into dormancy and did not result in commercially-sized daughter bulbs. Thus, it would be interesting to investigate whether the axil of the scales where ' $A$ and $B$ ' buds arise do have the competence to initiate more than one bud as well. For instance, it has been reported that a treatment with trichostatin A (TSA), a histone deacetylation inhibitor, together with the synthetic cytokinin BAP, in leaf axils of Arabidopsis, induces the expression of WUSCHEL (WUS), a gene required for the initiation of a shoot meristem (Wang et al. 2017). This result in Arabidopsis suggests that axillary bud initiation requires a permissive configuration of the chromatin so that WUS can be induced by a cytokinin pulse (Wang et al. 2017). In line with this hypothesis, it was shown previously that the induction of somatic embryogenesis by auxin, which also involves de novo meristem formation, can be significantly improved by TSA treatment (Li et al. 2014a). In order to predict whether similar mechanisms play a role in tulip, a deeper understanding of the initiation of tulip axillary meristem initiation is required. Moreover, the time window to induce the initiation of those buds has to be taken into consideration, since it might only work for a short period after the SAM has transitioned to the flowering state. 


\section{De novo bulblet regeneration in lily bulb scales, de-differentiation?}

The term de-differentiation in relation to regeneration has been questioned by some researchers e.g. (Sugimoto et al. 2011), because of the findings that callus, a tissue long time thought to be formed by de-differentiation, rather arises from pre-existing meristematic cells (pericycle) in Arabidopsis (Atta et al. 2009; Sugimoto et al. 2010). Although we cannot label pericycle cells in lily in order to trace their putative involvement in bulblet regeneration, we showed in chapter 5 and chapter $\mathbf{6}$ that regeneration arises from differentiated parenchyma cells. Moreover, we showed that those cells undergo massive and asymmetrical cell proliferation and lose their starch granules typical from parenchyma tissue, indicating that dedifferentiation took place.

Thus the high regeneration capacity of lily bulb scales could rely on their capacity to dedifferentiate into a competent tissue. In Arabidopsis, such competent tissue is callus. We did not find evidence for callus formation in lily explants (chapter 5 and 6), such as disorganized cell proliferation, neither expression of few root markers that are representative for the early stage of callus development (lkeuchi et al. 2013). However, we did find LoWOUND-INDUCED DEDIFFERENTIATION 2 (LOWIND2) highly expressed within one day after the start of culture in all explants, and it is known that callus induced by WIND genes does not display the typical root meristem markers (Iwase et al. 2011 a). Is then this de-differentiated and proliferated tissue a type of callus not described before? More targeted research in this direction would for sure shed light into the answer.

\section{De-differentiation, regeneration and epigenetic regulation}

Epigenetic regulation, which refers to heritable changes in gene expression and not caused by changes in the DNA sequence, include DNA modifications, such as DNA methylation and glycosylation; chromatin modifications such as histone methylation and acetylation; and small RNA-mediated regulation (Neelakandan and Wang 2012). There is increasing evidence of epigenetic regulation during regeneration (Tanaka et al. 2008; Ikeuchi et al. 2015; Li et al. 2011 b; Wang et al. 2017). For example, the DNA methyltransferase 1 mutant, met1, displays lower levels of DNA methylation and higher expression of WUS during regeneration. This mutation enhances the regeneration capacity and timing of regeneration compared to the wild-type (Li et al. 201 la). Moreover, the protein complex POLYCOMB REPRESSIVE COMPLEX2 (PRC2), which maintains the repressive chromatin state, is involved in preventing the reacquisition of stem cell fate in lateral organs of shoots (Lodha et al. 2013). Also, PRC2 targets genes involved in cell dedifferentiation such as WIND3 (Ikeuchi et al. 2016).

In chapter 5 it was indicated that LOSWINGER (LOSWR), which encodes a polycomb group protein, was highly induced within the first day after the start of culture and this expression increased further in time. Moreover, there were no differences in LoSWR expression level among 
the explants with different regeneration efficiency, indicating that this gene is necessary for regeneration but not causal of the differences in timing and number of regeneration events. SWR in Arabidopsis plays a major role in regeneration, more specifically in the capacity to regenerate callus from leaves (He et al. 2012). Thus, although we did not investigate in detail the expression of genes involved in epigenetic regulation, the expression pattern of LOSWR suggests that chromatin modification plays an important role in de-differentiation during de novo regeneration in lily bulb scales. In this sense, lily bulb scales are a good model system to investigate the bases of chromatin changes during de-differentiation and organ regeneration. In this PhD study one pilot experiment was performed in this direction, in which TSA treatment (the histone deacetylase inhibitor discussed above) was performed during the scaling process (data not included in this thesis). Under the tested conditions, this resulted in a block of regeneration instead of increased regeneration. Though, it might be that the treatment was not given at the right moment and furthermore, in depth analyses at the cellular level will be needed to investigate the exact effects of such a treatment and to understand the mode of action. Due to time constraints, we didn't follow up this pilot experiment, but in light of what is discussed above, it would be a good direction for future research.

\section{What is the trigger to induce ectopic bulblet regeneration?}

Wounding definitely triggers regeneration in lily and other species that have a native de novo regeneration capacity, such as begonia and crassula (Hartmann 2014). But wounding in this context refers to excising the explant completely from the mother plant. Interestingly, when lily explants are detached in a reactive oxygen species (ROS) scavenger solution and cultured in a medium containing ROS scavengers, regeneration does not occur and the survival of the explants is compromised (van Rossum 1997). This observation suggests that ROS is triggering a regeneration response, although we did not manage to prove this in chapter $\mathbf{5}$. However, wounding responses are highly diverse and their interconnection makes it difficult to dissect the true wounding signal that might activate regeneration.

Whatever this signal is, we proposed in chapter 5 and 6 that APETALA 2/ETHYLENE RESPONSE FACTORS (LOAP2/ERF) might be key integrators of wounding, induction of cell proliferation and de-differentiation and polar auxin redistribution. Nevertheless, more research is needed to prove this model. The implementation of a transient gene silencing protocol, as mentioned at the start of this chapter, would be of great help to understand the role of ERFs in the proposed regeneration model. For instance, it would be interesting to test whether the induced cell proliferation is e.g. mediated by LOWIND2 and/or LOERF1 15. Moreover, it is important to know whether polar auxin redistribution which is proposed to be a key initial process is mediated by LOERF109 or just simply a result of mechanical interruption of the auxin flow caused by the wound. However, it is necessary to first prove that polar auxin redistribution occurs, which is not a matter of course in lily because of the lack of stably integrated fluorescent markers for auxin 
distribution and auxin efflux carriers. A first start could be to use radioactive-labelled auxin to trace its transport by different tissues of the scale, such as main vascular bundles, parenchyma and epidermis. A similar approach is described by (Spicer et al. 2013) in order to address the identification of polar auxin transport in poplar.

Moreover, to elucidate further the gene networks that grant lily their high regeneration capacity, a more detailed transcriptome study could be done, in which the temporal and spatial resolution is improved. For example, a saturation of time points previous to the expression of LOESR2, a gene involved in shoot formation (lkeda et al. 2006; Matsuo et al. 2011) and proved to correlate with the regeneration capacity of the lily explants (Chapter 5) should be included. Those time points could be 0, 0.5, 2, 16 and 24 hours after excision and explant culture. Also, instead of using entire bulb scales, small explants of maximum $0.5 \mathrm{~cm}$ wide should be used, to make sure that only one bulblet is regenerated in the explant, to overcome a lot of variation in signal due to differences in number and timing of regenerating bulblets. Finally, to increase the resolution and to take the findings of chapter 6 into account, the small explant could be divided into four regions: adaxial epidermis, adaxial parenchyma, abaxial parenchyma and abaxial epidermis.

\section{Role of the epidermis in de novo bulblet regeneration in lily bulb scales}

As it was discussed in chapter 6 , the lily epidermis seems to enhance regeneration and to play a role positioning the niche where the ectopic meristem will regenerate. That place is normally at the adaxial and proximal side of the explant (Chapter 6). Unfortunately, we could not identify the regeneration stimulating compound in the epidermis by a classical tissue culture approach. Thus, a combined metabolomics, proteomic and transcriptomic approach in a time series during regeneration, would profoundly help unravelling the role of the epidermis in de novo regeneration and to identify the regenerating stimulating and positioning compound(s) produced in this cell layer.

\section{Lily bulb scales as a model to study regeneration recalcitrance in tulip?}

Because we aimed to understand and improve vegetative reproduction in lily and tulip, it is worth speculating about how the results obtained in lily could be transferred to recalcitrant tulip. There are two bottleneck aspects during in vitro regeneration in tulip. First, the limited amount of competent tissues and second, that regeneration results in shoots and not in bulbs (Flavell 2009; Alderson and Rice 1983; Alderson and Taeb 1990; Baker and Wilkins 1988; Maślanka and Bach 2014; Minas 2007; Podwyszyńska 2006; Podwyszynska and Sochacki 2010; Taeb and Alderson 1990b). Young floral stalks are the most commonly used explant in tulip because they bypass the callus stage and regenerate adventitious shoots under shoot induction medium (Alderson and Taeb 1990). However, the amount of explants that can be obtained from this tissue is very limited, a reason why the regenerated shoots are re-used for 
several rounds of regeneration in order to multiply the starting material (Podwyszynska and Sochacki 2010).

From a scientific perspective, it would be interesting to find out whether the low competence in tulip tissues is due to limited expression of the fast-induced integrators of regeneration observed in lily, e.g. LOAP2/ERFs (ERF115, WIND2 and ESR2); and chromatin remodelers such as LOSWR (Chapter 5). Although in the last case, more information about epigenetic control would have to be generated first from the efficient lily regeneration system.

From an applied perspective, the limited number of starting material for regeneration in tulip might be overcome by increasing the regeneration competence of e.g. scales, by treating them with chromatin remodelling targeting compounds. In line with this approach, using the two inner scale axils of bulbs around the time of transition to the reproductive state, as mentioned earlier, might result in axillary bulbs instead of shoots and as such, this would solve the second mentioned bottle neck. Finally, it would be interesting to test the putative regeneration enhancing function of lily scale epidermis when added to the growth medium of tulip explants, although for this it is probably the best to first get this technology optimally running in lily.

\section{Concluding remarks}

The aim of this thesis was to understand the mechanisms that control vegetative propagation in tulip and lily bulbs. Despite the challenges of studying a non-model species with very limited biotechnological resources and tools, this thesis set a physiological, cellular and molecular basis of how axillary bud outgrowth is controlled in tulip bulbs, and how regeneration capacity is established in lily bulb scales. Moreover, it triggers new research questions for both the fundamental and the applied communities. For further confirmation and thorough understanding of the described mechanisms it is necessary to invest in the development of biotechnological tools for gene function verification and life-monitoring of molecular processes in bulbs. Furthermore, based on the outcomes of this thesis and gained experiences, experimental set ups can be improved, and e.g. detailed hormone measurements and metabolomics approaches could be followed to investigate the complex sink-source relationships that play a pivotal role in vegetative propagation. 
Chapter 7 


\section{REFERNCES}


AGI (2000) Analysis of the genome sequence of the flowering plant Arabidopsis thalia na. 408 (6814):796-815

Aguilar-Martínez JA, Poza-Camón C, Cubas P (2007) Arabidopsis Branchedl acts as an integrator of branching signals within axillary buds. Plant Cell 19 (2):458-472. doi:10.1105/tpc.106.048934

Aida M, Ishida T, Tasaka M (1999) Shoot apical meristem and cotyledon formation during Arabidopsis embryogenesis: interaction among the CUP-SHAPED COTYLEDON and SHOOTMERISTEMLESS genes. Development 126 (8):1563-1570

Akita Y, Horikawa Y, Kanno A (2008) Comparative a nalysis of floral MADS-box genes between wild-type and a putative homeotic mutant in lily. J Hort Sc i Biotech 83:453-461

Alderson PG, Rice RD (1983) TOWARDS THE PROPAGATION OF TULP IN VITRO. Acta Hort (ISHS) 131:39-48

Alderson PG, Taeb AG (1990) Effect of bulb storage on shoot regeneration from floral stems of tulip in vitro. The J oumal of hortic ultural sc ience 65 (1):65-70

Alonso-Peral MM, Oliver SN, Casa o MC, Greenup AA, Trevaskis B (2011) The Promoter of the Cereal VERNALZATION1 Gene Is Suffic ient for Transcriptional Induction by Prolonged Cold. PLOS ONE 6 (12):e29456

Altschul SF, Gish W, Miller W, Myers EW, Lipman DJ (1990) Basic local alignment search tool. Joumal of Molecular Biology 215 (3):403-410. doi:http://dx.doi.org/10.1016/S00222836(05)80360-2

Alvarez J, Guli CL, Yu X-H, Smyth DR (1992) teminal flower: a gene affecting inflorescence development in Arabidopsis thaliana. The Plant Joumal 2 (1):103-116. doi:10.1111/j.1365-313X.1992.00103.x

Andres F, Coupland G (2012) The genetic basis of flowering responses to seasonal cues. 13 (9):627-639

Antonia di I, Plackova L, Simonovik B, Dolezal K, Tumbull C, Ljung K, Novak O (2015) Cell-TypeSpecific Cytokinin Distribution within the Arabidopsis Primary Root Apex. Plant Cell 27 (7):1955-1967. doi:10.1105/tpc.15.00176

APG III (2009) An update of the Ang iosperm Phylogeny Group classification for the orders and fa milies of flowering plants: APG III. Botanic alJ oumal of the Linnean Society 161 (2):105121. doi:10.1111/j.1095-8339.2009.00996.x

Askari N, Visser RGF, de Klerk GJ (2016) Advantageous effects of mild abiotic stresses in lily cultured in vitro

Propagation of Omamental Plants 16 (4):130-136

Atta R, La urens L, Boucheron-Dubuisson E, G uivarc'h A, Camero E, Giraudat-Pautot V, Rech P, Chriqui D (2009) Pluripotency of Arabidopsis xylem pericycle underlies shoot 
regeneration from root and hypocotyl explants grown in vitro. The Plant Joumal 57 (4):626-644. doi:10.1111/j.1365-313X.2008.03715.x

Aung LH, Hertogh AAD (1979) Temperature Regulation of Growth and Endogenous Abscisic Acid-like Content of Tulipa gesneriana L Plant Physiology 63 (6):1111-1116. doi:10.2307/4265806

Axtell MJ , SnyderJ A, Ba rtel DP (2007) Common Func tions for Diverse Sma ll RNAs of La nd Pla nts. The Plant Cell Online 19 (6):1750-1769. doi:10.1105/tpc.107.051706

Ayre BG (2011) Membrane-Transport Systems for Sucrose in Relation to Whole-Plant Carbon Partitioning. Molecular Plant 4 (3):377-394. doi:http://dx.doi.org/10.1093/mp/ssr014

Bach A, Sochacki D (2012) Propagation of Omamental Geophytes. In: Omamental Geophytes. CRC Press, pp 261-286. doi:doi:10.1201/b12881-11

BakerC, Wilkins H (1988) The influence of temperature intemuption on in-vitro tulip propagation. . HortSci 23 (780)

Balasubramanian S, Weigel D (2006) Temperature Induced Flowering in Arabidopsis thaliana. Plant Signa ling and Behavior 1 (5):227-228

Baldwin S, Revanna R, Pither-J oyce M, Shaw M, Wright K, Thomson S, Moya L, Lee R, Macknight R, McCallum J (2014) Genetic a nalyses of bolting in bulb onion (Allium cepa L.). TAG Theoretical and applied genetics Theoretisc he und angewandte Genetik 127 (3):535547. doi:10.1007/s00122-013-2232-4

Balk PA, de Boer AD (1999) Rapid stalk elongation in tulip (Tulipa gesneriana L. cv. Apeldoom) and the combined action of cold-induced invertase and the water-channel protein YTIP (Planta). 209 (3):346-354. doi:10.1007/s004250050642

Barba-Gonzalez R, Lim K-B, Ramanna M, Visser R, Van Tuyl J (2005) Occurrence of 2n gametes in the F 1 hybrids of Orientalx Asiatic lilies (Lilium): relevance to intergenomic recombination and backcrossing. Euphytica 143 (1):67-73

Barbier F, Péron T, Lecerf M, Perez-Garcia M-D, Barrière Q, Rolčík J, Boutet-Mercey S, Citeme S, Lemoine R, Porcheron B, Roman H, Leduc N, Le Goumierec J, Bertheloot J, Sa kr S (2015) Sucrose is an early modulator of the key hormonal mechanisms controlling bud outgrowth in Rosa hybrida. J Exp Bot 66 (9):2569-2582. doi:10.1093/jxb/en047

Bartoli CG, Casalongué CA, Simontacchi M, Marquez-Garcia B, Foyer CH (2013) Interactions between homone and redox signalling pathways in the control of growth and cross tolerance to stress. Environmental and Experimental Botany 94:73-88. doi:http://dx.doi.org/10.1016/j.envexpbot.2012.05.003

Beijer (1952) De ontwikkelingssta dia van tulp. La boratorium voor Bloembollenonderzoek 92:1-7 Benedito VA, Visser PB, Angenent GC, Krens FA (2004) The potential of virus-induced gene silencing for speeding up functional characterization of plant genes. Genetics and Molecular Research 3 (3):323-341 
Bennett T, Leyser O (2006) Something on the Side: Axillary Meristems and Plant Development. Plant Mol Biol 60 (6):843-854. doi:10.1007/s11103-005-2763-4

Benschop M, Kamenetsky R, Le Nard M, Okubo H, De Hertogh A (2010) The Global Flower Bulb Industry: Production, Utilization, Research. In: Horticultural Reviews. J ohn Wiley \& Sons, Inc., pp 1-115. doi:10.1002/9780470527238.ch1

Bihmidine S, Hunter C, Johns C, Koch K, Bra un D (2013) Regulation of a ssimilate import into sink organs: update on molecular drivers of sink strength. Frontiers in Plant Science 4 (177). doi:10.3389/fpls.2013.00177

Bindschedler LV, Cramer R (2011) Quantitative plant proteomics. Proteomics 11 (4):756-775. doi:10.1002/pmic .201000426

Blancaflor EB, Fasano J M, Gilroy S (1998) Mapping the functional roles of cap cells in the response of Arabidopsis primary roots to gravity. Plant Physiol 116 (1):213-222

Blilou I, Xu J , Wild water M, Willemsen V, Paponov I, Friml J (2005) The PIN a uxin efflux facilitator network controls growth and patteming in Arabidopsis roots. Nature 433 (null):39

Bolger AM, Lohse M, Usadel B (2014a) Trimmomatic: a flexible trimmer for Illumina sequence data. Bio informatics 30 (15):2114-2120. doi:10.1093/bio informatic s/btu170

Bolger AM, Lohse M, Usa del B (2014b) Trimmomatic: A flexible trimmer for lllumina Sequence Data. Bioinformatics. doi:10.1093/bioinformatics/btu170

Bonnier FJ M, Van Tuyl J M (1997) Long tem t in vitro storage of lily: effects of temperature and concentration of nutrients and sucrose. Plant Cell Tissue Organ Cult 49 (2):81-87. doi:10.1023/a:1005810728215

Booker J, Sieberer T, Wright W, Williamson L, Willett B, Stimberg P, Tumbull C, Srinivasan M, Goddard P, Leyser O (2005) MAXI encodes a cytochrome P450 family member that acts downstream of $M A X 3 / 4$ to produce a carotenoid-derived branch-inhibiting homone. Developmental Cell 8 (3):443-449

Bra dy SM, Orlando DA, Lee JY, Wang JY, Koch J, Dinneny J R, Mace D, O hlerU, Benfey PN (2007) A high-resolution root spatiotemporal map reveals dominant expression pattems. 318:801-806

Braun N, de Sa int Germa in A, Pillot J -P, Boutet-Mercey S, Dalma is M, Antonia di I, L X, MaiaGrondard A, Le Signor C, Bouteiller N, Luo D, Bendahmane A, Tumbull C, Rameau C (2012a) The Pea TCP Transc ription Fac tor PsBRC 1 Acts Downstream of Strigolac tones to Control Shoot Branching. Plant Physiology 158 (1):225-238. doi:10.1104/pp.111.182725

Bra un N, Germa in AS, Pillot J P, Boutet-Mercey S, Da Ima is M, Antonia di I, L X, Maia-Grondard A, le Signor C, Bouteiller N, Luo D, Bendahmane A, Tumbull C, Rameau C (2012b) The pea TCP transcription factor PSBRC 1 acts downstream of strigolactones to control shoot branching. Plant Physiology 158 (1):225-238. doi:10.1104/pp.111.182725

Bray NL, Pimentel H, Melsted P, Pachter L (2016) Near-optimal probabilistic RNA-seq quantification. 34 (5):525-527 
Brewer PB, Dun EA, Ferguson BJ, Rameau C, Beveridge CA (2009) Strigolactone Acts Downstream of Auxin to Regulate Bud Outgrowth in Pea and Arabidopsis. Plant Physiology 150 (1):482-493

Buchfink B, Xie C, Huson DH (2015) Fast and sensitive protein alignment using DIAMOND. 12 (1):59-60

Cai X, Xu p, Zha o P, Lu R, YU L, Xiang C (2014) Ara bid opsis ERF109 media tes cross-talk between jasmonic acid and a uxin biosynthesisduring lateral root formation. Nat Commun 5:5833. doi:doi: 10.1038/ncomms6833.

Cassells AC, Momish FM (1985) Growth measurements of Begonia rex Putz. plants regenerated from leaf cuttings and in vitro from leaf petioles, axenic leaves, re-cycled axenic leaves and callus. Scientia Horticulturae 27 (1):113-121. doi:http://dx.doi.org/10.1016/03044238(85)90061-5

Chang Z, L G, Lu J, Zhang Y, Ashby C, Lu D, Cramer C, Huang X (2015) Bridger: a new framework for de novo transcriptome assembly using RNA-seq data. Genome Biology $16(1): 30$

Chatfield SP, Capron R, Severino A, Penttila P-A, Alfred S, Nahal H, Provart NJ (2013) Incipient stem cell nic he conversion in tissue culture: using a systems approach to probe early events in WUSCHEL-dependent conversion of lateral root primordia into shoot meristems. The Plant J oumal 73 (5):798-813. doi:10.1111/tpj.12085

Chatfield SP, Raizada MN (2008) Ethylene and shoot regeneration: hooklessl modulates de novo shoot organogenesis in Arabidopsis thaliana. Plant Cell Rep 27 (4):655-666. doi:10.1007/s00299-007-0496-3

Chatfield SP, Stimberg P, Forde BG, Leyser O (2000) The homonal regulation of axillary bud growth in Arabidopsis. Plant J oumal 24 (2):159-169

Che P, Lall S, Howell SH (2007) Developmental steps in acquining competence for shoot development in Ara bidopsistissue culture. Planta 226 (5):1183-1194. doi:10.1007/s00425007-0565-4

Chen M-K, Lin I-C, Yang C-H (2008) Functional Analysis of Three Lily (Lilium longiflorum) APETALA1-like MADSBox Genes in Regulating Floral Transition and Formation. Plant and Cell Physiology 49 (5):704-717. doi:10.1093/pcp/pcn046

Cheng Z, Wang L, Sun W, Zhang Y, Zhou C, Su YH, L W, Sun TT, Zhao XY, L XG, Cheng Y, Zha o $Y$, Xie Q, Zhang XS (2013) Pattem of a uxin and cytokinin responses for shoot meristem induction results from the regulation of cytokinin biosynthesis by AUXIN RESPONSE FACTOR3. Plant Physiol 161 (1):240-251. doi:10.1104/pp.112.203166

Choi K, Kim J, Hwang H-J , Kim S, Park C, Kim SY, Lee I (2011) The FRIG IDA Complex Activates Transcription of FLC, a Strong Flowering Repressor in Arabidopsis, by Recruiting Chromatin Modification Factors. The Plant Cell Online 23 (1):289-303. doi:10.1105/tpc.110.075911 
Cline MG (1994) The role of homones in apical dominance. New approaches to an old problem in plant development. Physiologia Plantarum 90 (1):230-237. doi:10.1111/j.1399-3054.1994.tb02216.x

Cline MG (1996) Exogenous auxin effects on lateral bud outgrowth in decapitated shoots. Annals of Botany 78 (2):255-266

Cline MG (2000) Execution of the Auxin Replacement Apical Dominance Experiment in Temperate Woody Species. American Joumal of Botany 87 (2):182-190. doi:10.2307/2656904

Cline MG, Chatfield SP, Leyser O (2001) NAA Restores Apic al Dominance in the axr3-1 Mutant of Ara bidopsis tha liana. Annals of Botany 87 (1):61-65. doi:10.1006/anbo.2000.1298

Cohen A, Meredith CP (1992) AG ROBAC TERIUM - MEDIATED TRANSFO RMATION OF UUUM. Ac ta Hortic ulture 325:611-618

Consortium TU (2015) UniProt: a hub for protein information. Nucleic Acids Research 43 (D1):D204-D212. doi:10.1093/nar/gku989

Cooke JEK, Eriksson ME, Junttila O (2012) The dynamic nature of bud domancy in trees: environmental control and molec ularmec hanisms. Plant Cell Environ 35 (10):1707-1728. doi:10.1111/j.1365-3040.2012.02552.x

Da nisman S, van Dijk ADJ , Bimbo A, van derWal F, Hennig L, de Folter S, Angenent GC, Immink RGH (2013) Analysis of functional redundancies within the Ara bid opsis TCP transcription factor fa mily. J oumal of Experimental Botany. doi:10.1093/jxb/ert337

David Law R, Suttle JC (2004) Changes in histone H3 and H4 multi-acetylation during natural and forced domancy break in potato tubers. Physiologia Plantarum 120 (4):642-649. doi:10.1111/j.0031-9317.2004.0273.x

De Hertogh A, Le Nard M (1993a) The Physiology of Flower Bulbs: A Comprehensive Treatise on the Physiology and Utilization of Omamental Flowering Bulbous and Tuberous Plants. Elsevier Science,

De Hertogh AA, Aung LH, Benschop M (1983) The Tulip: Botany, Usage, Growth, and Development. In: Horticultural Reviews. John Wiley \& Sons, Inc., pp 45-125. doi:10.1002/9781118060728.ch2

De Hertogh AA, Le Nard M (1993b) Botanic al aspects of flower bulbs. In: De Hertogh AA, Le Nard M (eds) The physiology of flowering bulbs. Elsevier, Burlington, MA, USA, pp 7-28

De Schepper V, De Swaef T, Bauweraerts I, Steppe K (2013) Phloem transport: a review of mechanismsand controls. J Exp Bot 64 (16):4839-4850

De Villiers SM, Kamo K, Thomson JA, Bomman CH, Berger DK (2000) Biolistic transformation of chincherinchee (Omithogalum) and regeneration of transgenic plants. Physiologia Plantarum 109 (4):450-455. doi:10.1034/j.1399-3054.2000.100412.x 
Deng X (2011) SeqGene: a comprehensive software solution for mining exome- and transcriptome- sequencing data. BMC Bioinformatics 12:267-267. doi:10.1186/14712105-12-267

Diaz-Riquelme J , Lijavetzky D, Martinez-Zapater J , Ca mona M (2009) Genome-wide a na lysis of MIKCC-type MADS-box genes in grapevine. Plant Physiol 149:354 - 369

Doebley J, Stec A, Hubbard L (1997) The evolution of apical dominance in maize. 386 (6624):485-488

Domagalska MA, Leyser O (2011) Signal integration in the control of shoot branching. 12 (4):211-221

Duangjit J, Bohanec B, Chan AP, Town CD, Havey MJ (2013) Transcriptome sequencing to produce SNP-based genetic maps of onion. Theoretical and Applied Genetics 126 (8):2093-2101. doi:10.1007/s00122-013-2121-x

Dubrovsky JG, Rost TL (2001) Pericycle. In: eLS. John Wiley \& Sons, Ltd. doi:10.1002/9780470015902.a0002085.pub2

Dubrovsky J G, Sa uerM, Napsucia ly-Mendivil S, Ivanchenko MG , Friml J , Shishkova S(2008) Auxin acts as a local morphogenetic trigger to specify lateral root founder cells. Proc Natl Acad SciUSA 105 (null):8790

Duclercq J , Sangwan-Norreel B, Catterou M, Sangwan RS (2011) De novo shoot organogenesis: from art to science. Trends in Plant Science 16 (11):597-606. doi:http://dx.doi.org/10.1016/j.tplants.2011.08.004

Dun EA, de Saint Germa in A, Rameau C, Beveridge CA (2012) Antagonistic Action of Strigolactone and Cytokinin in Bud Outgrowth Control. Plant Physiology 158 (1):487-498. doi:10.1104/pp.111.186783

Elfving DC, Visser DB, Henry J L (2011) Gibberellins Stimulate Lateral Branch Development in Young Sweet Chemy Trees in the Orchard. Intemational Joumal of Fruit Science 11 (1):41-54. doi:10.1080/15538362.2011.554066

Emms D, Kelly S (2015) OrthoFinder. solving fundamental biases in whole genome comparisons dramatically improves orthogroup inference accuracy. Genome Biology 16 (1):1-14. doi:10.1186/s13059-015-0721-2

Ferguson BJ , Beveridge CA (2009) Roles for auxin, cytokinin, and strigolactone in regulating shoot branching1[C][W][OA]. Plant Physiology 149 (4):1929-1944. doi:10.1104/pp.109.135475

Ferra rio S, Immink RG H, Angenent GC (2004) Conservation and diversity in flowerland. Current Opinion in Plant Biology 7 (1):84-91. doi:http://dx.doi.org/10.1016/j.pbi.2003.11.003

Finlayson SA (2007) Arabidopsis TEOSINTE BRANCHED1-LKE 1 Regulates Axillary Bud Outgrowth and is Homologous to Monocot TEOSINTE BRANCHED1. Plant and Cell Physiology 48 (5):667-677. doi:10.1093/pcp/pc m044 
Finn RD, Bateman A, Clements J, Coggill P, Eberhardt RY, Eddy SR, Heger A, Hetherington K, Holm L, Mistry J, Sonnhammer EL, Tate J, Punta M (2014) Pfam: the protein families database. Nucleic Acids Research 42 (D1):D222-D230. doi:10.1093/nar/gkt1223

Flavell R (2009) Role of model plant species. MethodsMol Biol 513:1-18. doi:10.1007/978-1-59745427-8_1

Fomara F, Coupland G (2009) Plant Phase Transitions Make a SPLash. Cell 138 (4):625-627. doi:http://dx.doi.org/10.1016/j.cell.2009.08.011

Foyer CH, Noctor GCFpdJ (2005) Redox Homeostasis and Antioxidant Signaling: A Metabolic Interface between Stress Perception and Physiological Responses. The Plant Cell 17 (7):1866-1875

Friml J (2003) Auxin transport -- shaping the plant. 6:7-12

Friml J, Wiśniewska J, Benková E, Mendgen K, Palme K (2002) Lateral relocation of auxin efflux regula tor PIN3 mediates tropism in Arabidopsis. Nature 415 (null):806

Fukaki H, Wysocka-Diller J, Ka to T, Fujisawa H, Benfey PN, Tasaka M (1998) Genetic evidence that the endodermis is essential for shoot gra vitropism in Arabidopsis tha lia na. Plant J 14 (4):425-430

Galvão VC, Nordström KJV, Lanz C, Sulz P, Mathieu J, Posé D, Schmid M, Weigel D, Schneeberger K (2012) Synteny-based mapping-by-sequencing enabled by targeted enrichment. The Plant J oumal 71 (3):517-526. doi:10.1111/j.1365-313X.2012.04993.x

Garber M, Grabherr MG, Guttman M, Trapnell C (2011) Computational methods for transc riptome a nnotation and quantific ation using RNA-seq. Nat Meth 8 (6):469-477

Garcês HMP, Champagne CEM, Townsley BT, Park S, Malhó R, Pedroso MC, Harada JJ , Sinha NR (2007) Evolution of asexual reproduction in leaves of the genus Kalanchoë. Proceedings of the National Academy of Sciences 104 (39):15578-15583. doi:10.1073/pnas.0704105104

Gargioli C, SlackJ M (2004) Cell lineage tracing during Xenopusta il regeneration. Development 131 (11):2669-2679. doi:10.1242/dev.01155

Gaud in V, Lunness PA, Fobert PR, Towers M, Riou-Kha mlichi C, Murray J AH, Coen E, Doonan J H (2000) The Expression of D-Cyclin Genes Defines Distinct Developmental Zones in Snapdragon Apic al Meristems and Is Locally Regulated by the Cycloidea Gene. Plant Physiology 122 (4):1137-1148

Gemits MM, de Klerk GJ (1992) Dry-matter partitioning between bulbs and leaves in pla ntlets of Lilium speciosum regenerated in vitro. Acta Botanica Neerlandica 41 (4):461-468. doi:10.1111/j.1438-8677.1992.tb 00516.x

Godwin J W, Pinto AR, Rosenthal NA (2013) Macrophages a re required for adult salamander limb regeneration. Proceedings of the National Academy of Sciences 110 (23):94159420. doi:10.1073/pnas.1300290110 
Gomez-Roldan V, Femas S, Brewer PB, Puech-Pagès V, Dun EA, Pillot J P, Letisse F, Matusova R, Danoun S, Porta is J C, Bouwmeester H, Bécard G, Beveridge CA, Ramea u C, Rochange SF (2008) Strigolactone inhibition of shoot branching. Nature 455 (7210):189-194. doi:10.1038/nature07271

González-Grandío E, Cubas P (2014) Identific a tion of gene functions associated to ac tive and domant buds in Arabidopsis. Plant Signaling \& Behavior 9:e27994. doi:10.4161/psb.27994

González-Grandío E, Pajoro A, Franco-Zomilla J M, Tarancón C, Immink RGH, Cubas P (2017) Abscisic acid signaling is controlled by a BRANCHED1/HD-ZP I casc ade in Arabidopsis axillary buds. Proceedings of the National Academy of Sciences 114 (2):E245-E254. doi:10.1073/pnas.1613199114

González-Grandío E, Poza-Camón C, Sorzano COS, Cubas P (2013) BRANCHED1 Promotes Axillary Bud Domancy in Response to Shade in Arabidopsis. The Plant Cell Online. doi:10.1105/tpc.112.108480

Gordon SP, Heisler MG, Reddy GV, Ohno C, Das P, Meyerowitz EM (2007) Pattem formation during de novo assembly of the $<e m>$ Arabidopsis $</$ em $>$ shoot meristem. Development 134 (19):3539-3548. doi:10.1242/dev.010298

Grabherr MG, Haas BJ, Yassour M, Levin JZ, Thompson DA, Amit I, Adiconis X, Fan L, Raychowdhury R, Zeng Q, Chen Z, Mauceli E, Hacohen N, G nirke A, Rhind N, di Palma F, Birren BW, Nusbaum C, Lindblad-Toh K, Friedman N, Regev A (2011a) Full-length transc riptome assembly from RNA-Seq data without a reference genome. 29 (7):644652

Grabherr MG, Haas BJ, Yassour M, Levin JZ, Thompson DA, Amit I, Adiconis X, Fan L, Raychowdhury R, Zeng Q, Chen Z, Mauceli E, Hacohen N, Gnirke A, Rhind N, di Palma F, Birren BW, Nusbaum C, Lindblad-Toh K, Friedman N, Regev A (2011b) Trinity: reconstructing a full-length transcriptome without a genome from RNA-Seq data. Nature biotechnology 29 (7):644-652. doi:10.1038/nbt.1883

Grbić V, Bleecker AB (2000) Axillary meristem development in Arabidopsis thaliana. The Plant J oumal 21 (2):215-223. doi:10.1046/j.1365-313x.2000.00670.x

Greb T, Clarenz O, Schäfer E, Müller D, Herrero R, Sc hmitz G, Theres K (2003) Molecular a nalysis of the LATERAL SUPPRESSOR gene in Arabidopsis reveals a conserved control mechanism for axillary menistem formation. Genes and Development 17 (9):1175-1187. doi:10.1101/gad.260703

Greenup AG, Sasani S, Oliver SN, Talbot MJ, Dennis ES, Hemming MN, Trevaskis B (2010) ODDSO C2 Is a MADS Box Floral Repressor That Is Down-Regulated by Vemalization in Temperate Cereals. Plant Physiology 153 (3):1062-1073. doi:10.1104/pp.109.152488

Haas BJ , Papanic ola ou A, Yassour M, Grabherr M, Blood PD, Bowden J, Couger MB, Ec cles D, L B, Lieber M, MacManes MD, Ott M, Orvis J, Pochet N, Strozzi F, Weeks N, Westeman 
R, William T, Dewey CN, Henschel R, LeDuc RD, Friedman N, Regev A (2013a) De novo transcript sequence reconstruction from RNA-seq using the Trinity platform for reference generation and analysis. 8 (8):1494-1512

Haas BJ , Papanic olaou A, Yassour M, Grabherr M, Blood PD, Bowden J, Couger MB, Ec cles D, L B, Lieber M, MacManes MD, Ott M, Orvis J, Pochet N, Strozzi F, Weeks N, Westeman R, William T, Dewey CN, Hensc hel R, LeDuc RD, Friedman N, Regev A (2013b) De novo transcript sequence reconstruction from RNA-Seq: reference generation and analysis with Trinity. Nature protocols 8 (8):10.1038/nprot.2013.1084. doi:10.1038/nprot.2013.084

Hackett W (1969) Aseptic multiplication of lily bulblets from bulb scales Proc Int Plant Prop Soc 19:105-108

Ha rtmann A, Senning M, Hed den P, Sonnewa ld U, Sonnewald S(2011) Rea ctivation of Meristem Activity and Sprout Growth in Potato Tubers Require Both Cytokinin and Gibberellin. Plant Physiology 155 (2):776-796. doi:10.1104/pp.110.168252

Hartmann HT(2014) Hartmann \& Kester's plant propagation principles and practices. Pearson, Harlow

Hartsema AM (1961) Influence of temperature on flower fomation and flowering of bulbous and tuberousplants. Encyclopedia of Plant Physiology 16, ed. W. Ruhland 123-167 Berlin: Springer verslag

He C, Chen X, Huang H, Xu L (2012) Reprogra mming of H3K27me3 Is Critic al for Acquisition of Plunipotency from Cultured Ara bid opsis Tissues. PLOS Genetic s 8 (8):e1002911

Heide OM (1968) Auxin level and regeneration of Begonia leaves. Planta 81 (2):153-159. doi:10.1007/bf00417444

Henry C, Rabot A, La loi M, Mortreau E, Sigogne M, Leduc N, Lemoine R, SakrS, Vian A, PelleschiTravier S (2011) Regulation of RhSUC2, a sucrose transporter, is correlated with the light control of bud burst in Rosa sp. Plant, Cell \& Environment 34 (10):1776-1789. doi:10.1111/j.1365-3040.2011.02374.x

Heyman J, Cools T, Canher B, Shavialenka S, Traas J, Vercauteren I, Van den Daele H, Persiau G, De J a eger G, Sugimoto K, De Veylder L (2016) The heterodimeric transcription factor complex ERF115-PATI grants regeneration competence. 2:16165

Heyman J, Cools T, Vandenbussche F, Heyndrickx KS, Van Leene J, Vercauteren I, Vanderauwera S, Vandepoele K, De J aegerG, Van DerStraeten D, De Veylder L (2013) ERF115 Controls Root Quiescent Center Cell Division and Stem Cell Replenishment. Science 342 (6160):860-863. doi:10.1126/science.1240667

Ho LC, Rees AR (1975) Aspects of Translocation of Carbon in the Tulip. The New Phytologist 74 (3):421-428

Ho LC, ReesAR (1976) Re-Mobilization and Redistribution of Reserves in the Tulip Bulb in Relation to New Growth Until Anthesis. The New Phytologist 76 (1):59-68 
Ho LC, Rees AR (1977) The Contribution of Current Photosynthesis to G rowth and Development in the Tulip During Flowering. The New Phytologist 78 (1):65-70

Hooykaas PJ J , Schilperoort RA (1992) Agrobacterium and plant genetic eng ineering. Plant Mol Biol 20 (1):175-175. doi:10.1007/bf00029165

Horvath D (2009) Common mechanisms regulate flowering and domancy. Plant Science 177:523-531

Horvath D, Anderson J , Chao W, Foley M (2003) Knowing when to grow: signals regulating bud domancy. Trends Plant Sci 8:534 - 540

Hou R, Bao Z, Wang S, Su H, L Y, Du H, Hu J, Wang S, Hu X (2011) Transcriptome Sequencing and $\langle$ talic $>$ De Novo $<$ italic $>$ Analysis for Yesso Scallop ( $<$ talic>Patinopecten yessoensis $<$ ita lic >) Using 454 G S FLX. PLoSONE 6 (6):e21560

Huang J , Chen F, Del Casino C, Autino A, Shen M, Yuan S, Peng J , Shi H, Wang C, Cresti M, L Y (2006) An Ankyrin Repeat-Containing Protein, Characterized as a Ubiquitin Ligase, Is Closely Associated with Membrane-Enclosed Organelles and Required for Pollen Germination and Pollen Tube Growth in Lily. Plant Physiology 140 (4):1374-1383. doi:10.1104/pp.105.074922

Hubbard L, McSteen P, Doebley J , Hake S (2002) Expression pattems and mutant phenotype of teosinte branched 1 correlate with growth suppression in maize and teosinte. Genetics 162 (4):1927-1935

Hulscher M, Krijgsheld HT, van der Linde PCG (1992) PROPAGATION OF SHOOTS AND BULB GROWTH OF TULP IN VITRO Acta Hort (ISHS) 325:441-446

Hunt M, Kikuchi T, Sanders M, Newbold C, Bemiman M, Otto T(2013) REAPR: a universal tool for genome assembly evaluation. Genome Biology 14 (5):R47

Huson DH, Auch AF, Qi J , Schuster SC (2007) MEGAN a nalysis of metagenomic data. Genome Research 17 (3):377-386. doi:10.1101/gr.5969107

Ikeda Y, Banno H, Niu Q-W, Howell SH, Chua N-H (2006) The ENHANCER OF SHOOT REGENERATION 2 gene in Arabidopsis Regulates CUP-SHAPED COTYLEDON 1 at the Transcriptional Level and Controls Cotyledon Development. Plant and Cell Physiology 47 (11):1443-1456

Ikegami M, Whigham DF, Werger MJ A (2012) Effects of local density of clonal plants on their sexual and vegetative propagation strategies in a lattice structure model. Ecological Modelling 234:51-59. doi:http://dx.doi.org/10.1016/j.ecolmodel.2012.03.026

Ikeuchi M, Iwase A, Sugimoto $K$ (2015) Control of plant cell differentiation by histone modification and DNA methylation. Current Opinion in Plant Biology 28:60-67. doi:http://dx.doi.org/10.1016/i.pbi.2015.09.004

Ikeuchi M, Ogawa Y, Iwase A, Sugimoto K (2016) Plant regeneration: cellular origins and molecular mechanisms. Development 143 (9):1442-1451. doi:10.1242/ dev.134668 
Ikeuchi M, Sugimoto K, Iwase A (2013) Plant Callus: Mechanisms of Induction and Repression. The Plant Cell 25 (9):3159-3173. doi:10.1105/tpc.113.116053

Initiative TAG (2000) Analysis of the genome sequence of the flowering plant Arabidopsis thaliana. 408 (6814):796-815

Ishikawa S, Maekawa M, Arite T, Onishi K, Taka mure I, Kyozuka J (2005) Suppression of tiller bud ac tivity in tillering dwa rf mutants of rice. Plant and Cell Physiology 46 (1):79-86. doi:C ited By (since 1996) 117

Export Date 30 J anuary 2013

Iwase A, Mitsuda N, Koyama T, Hiratsu K, Kojima M, Arai T, Inoue Y, Seki M, Sakakibara H, Sugimoto K, Ohme-Takagi M (2011a) The AP2/ERF Transc ription Factor WIND1 Controls Cell Dedifferentiation in Arabidopsis. Current Biology 21 (6):508-514. doi:http://dx.doi.org/10.1016/j.cub.2011.02.020

Iwase A, Ohme-Takagi M, Sugimoto K (2011b) WIND1: A key molecular switch for plant cell dedifferentiation. Plant Signaling \& Behavior 6 (12):1943-1945

Izawa T, Takahashi Y, Yano M (2003) Comparative biology comes into bloom: genomic and genetic comparison of flowering pathways in rice and Arabidopsis. Current Opinion in Plant Biology 6 (2):113-120. doi:http://dx.doi.org/10.1016/S1369-5266(03)00014-1

Jásik J, de Klerk G-J (2006) Effect of Methyl Jasmonate on Morphology and Domancy Development in Lily Bulblets Regenerated In Vitro. J oumal of Plant Growth Regulation 25 (1):45-51. doi:10.1007/s00344-005-0048-4

J a velle M, Vemoud V, Rogowsky PM, Ingra m GC (2011) Epidermis: the formation and functions of a fundamental plant tissue. New Phytologist 189 (1):17-39. doi:10.1111/j.14698137.2010.03514.x

J iang B, Miao H, Chen S, Zhang S, Chen F, Fang W (2009) The Lateral Suppressor-Like Gene, DgLSL, Altemated the Axillary Branching in Transgenic Chrysanthemum (Chrysanthemum $\times$ morifolium) by Modulating IAA and GA Content. Plant Molecular Biology Reporter 28 (1):144. doi:10.1007/s11105-009-0130-3

Jones Sl, Tan Y, Shamimuzzaman M, George S, Cunningham BT, Vodkin L (2015) Direct Detection of Transcription Factors in Cotyledons duning Seedling Development Using Sensitive Silic on-Substrate Photonic Crystal Protein Arrays. Plant Physiology 167 (3):639649. doi:10.1104/pp.114.253666

Kalivas A, Pasentsis K, Polidoros A, Tsaftaris A (2007) Heterotopic expression of B-class floral homeotic genes PISTLATA/GLOBOSA supports a modified model for crocus (Crocus sativus L.) flower formation. DNA sequence 18 (2):120-130

Kamei CLA, Severing El, Dechesne A, Furrer H, Dolstra O, Trindade LM (2016) Orphan Crops Browser: a bridge between model and orphan crops. Molecular Breeding 36 (1):1-18. doi:10.1007/s11032-015-0430-2 
Kamenetsky R (2012) Biodiversity of Geophytes- Phytogeography, Morphology, and Survival Strategies. In: Omamental Geophytes: From Basic Science to Susta inable Production. CRC Presspp 57-76. doi:doi:10.1201/b12881-4

Kamenetsky R, Fa igenboim A, Shemesh Mayer E, Ben Mic ha el T, Gershberg C, Kimhi S, Esquira I, Rohkin Shalom S, Eshel D, Ra binowitch HD, Sheman A (2015) Integrated tra nsc riptome catalogue and organ-specific profiling of gene expression in fertile garlic (Allium sativum L). BMC Genomics 16 (1):12. doi:10.1186/s12864-015-1212-2

Kamenetsky R, Okubo H (2013) Omamental geophytes : from basic science to susta inable production. CRC Press, Boca Raton, Fla [etc.]

Kamenetsky R, Zaccai M, Fla ishman MA (2012) Florogenesis. In: Ka menetsky R, Okubo H (eds) O mamental G eophytes. CRC Press. doi:10.1201/b12881-9

Ka mo K, Blowers A, Smith F, Van EckJ , Lawson R (1995) Sta ble Transformation of G ladiolus Using Suspension Cells and Callus. J oumal of the American Society for Horticultural Science $120(2): 347-352$

Kane NA, DanylukJ , Ta rd if G, O uellet F, La liberté J -F, Limin AE, Fowler DB, Sa rhan F (2005) Ta VRT2, a Member of the StMADS-11 Clade of Flowering Repressors, Is Regulated by Vemalization and Photoperiod in Wheat. Plant Physiology 138 (4):2354-2363. doi:10.1104/pp.105.061762

Kanno A, Sa eki H, Kameya T, SaedlerH, Theissen G (2003) Heterotopic expression of class B floral homeotic genes supports a modified ABC model for tulip (Tulipa gesneriana) (Plant Molecular Biology). 52 (4):831-841. doi:10.1023/a:1025070827979

Kareem A, Durgaprasad K, Sugimoto K, Du Y, Pulianmackal Ajaij, Trivedi Zankhana B, Abhayadev PazhoorV, Pinon V, Meyerowitz Elliot M, Scheres B, Prasad K (2015) PLETHO RA Genes Control Regeneration by a Two-Step Mechanism. Current Biology 25 (8):1017-1030. doi:http://dx.doi.org/10.1016/j.cub.2015.02.022

Ka ufmann K, Smaczniak C, de Vries S, Angenent G C, Karlova R (2011) Proteomic s insights into plant signaling and development. Proteomics 11 (4):744-755. doi:10.1002/pmic.201000418

Kebrom TH, Spielmeyer W, Finnegan EJ (2013) Grasses provide new insights into regulation of shoot branching. Trends in Plant Science 18 (1):41-48. doi:http://dx.doi.org/10.1016/j.tplants.2012.07.001

Keller T, Abbott J , Moritz T, Doemer P (2006) Arabid opsis REG ULATOR OF AXILARY MERISTEMSI controls a leaf axil stem cell niche and modulates vegetative development. Plant Cell 18 (3):598-611. doi:10.1105/tpc.105.038588

Kerstetter RA, Hake S (1997) Shoot menistem formation in vegetative development. Pla nt Cell 9 (7):1001-1010. doi:10.1105/tpc .9.7.1001

Khodorova N, Boitel-Conti M (2013) The Role of Temperature in the Growth and Flowering of Geophytes. Plants 2 (4):699 
Kim K-W, De Hertogh AA (2010) Tissure Culture of Omamental Flowering Bulbs (Geophytes). In: Hortic ultural Reviews. John Wiley \& Sons, Inc., pp 87-169. doi:10.1002/9780470650608.ch2

Klyne MA, Phan CT (1976) Morphological and biochemical effects of ethylene on Tulips. Experientia 32 (8):1004-1006. doi:10.1007/bf01933937

Koumpetis YAl, van Dijk ADJ, van Ham RCHJ, ter Braak CJF (2011) Genome-Wide Computational Function Prediction of Arabidopsis Proteins by Integration of Multiple Data Sources. Plant Physiology 155 (1):271-281. doi:10.1104/pp.110.162164

Kukułczanka K, Suszyńska G (1972) Regenerative properties of Saintpaulia ionantha Wendl. Lea ves cultured in vitro Acta Soc ieta tis Bota nic orum Polonia e 41:503-509

Kumar SV, Lucyshyn D, Jaeger KE, Alos E, Alvey E, Harberd NP, Wigge PA (2012) Transc ription factor PIF4 controls the themosensory a ctivation of flowering. 484 (7393):242-245

Kurakawa T, Ueda N, Maekawa M, Kobaya shi K, Kojima M, Nagato Y, Sa ka kibara H, Kyozuka J (2007) Direct c ontrol of shoot meristem activity by a cytokinin-activating enzyme. 445 (7128):652-655

Lambrechts H, Rook F, KollöffelC (1994) Carbohydrate Status of Tulip Bulbsduring Cold-Induced Flower Stalk Elongation and Flowering. Plant Physiology 104 (2):515-520. doi:10.2307/4275648

Lang A (1952) Physiology of flowering. Annual Revieuw of Plant Physiology 3:265-306

Lang G, Early J, Martin G, Damell R (1987) Endodomancy, paradomancy, and ecodomancy - physiological teminology and classification for domancy research. HortScience 22:371 - 377

Langens-Gemits M, Lilien-Kipnis H, Croes T, Miller W, Kollöffel C, de Klerk G-J (1997) BULB GROWTH IN LLY REG ENERA TED IN VITRO. Ac ta Hort (ISHS) 430:267-274

Langens-Gemits MM (2003) Phase change, bulb growth and domancy development in lily : manipulation of the propagation cycle by in vitro culture. Katholieke Universiteit Nijmegen,

Le Nard M, de Hertogh AA (1993) TUUPA. In: de Hertogh AA, Le Nard M (eds) The physiology of flowering bulbs. Elsevier, , Burlington, MA, USA, pp 617-682

Lee J-Y, Colinas J, Wang JY, Mace D, Ohler U, Benfey PN (2006) Transcriptional and posttranscriptional regulation of transcription factor expression in Arabidopsis roots. Proceedings of the National Academy of Sciences 103 (15):6055-6060. doi:10.1073/pnas.0510607103

Leeggangers HA, Moreno-Pachon N, Gude H, Immink RG (2013) Transfer of knowledge about flowering and vegetative propagation from model species to bulbous plants. The Intemational joumal of developmental biology 57 (6-8):611-620. doi:10.1387/ijdb.130238ri 
L B, Dewey CN (2011) RSEM: accurate transcript quantification from RNA-Seq data with or without a reference genome. BMC Bio informatic s 12:323-323. doi:10.1186/1471-2105-12323

L H, Soria no M, Cordewener J, Muiño J M, Riksen T, Fukuoka H, Angenent GC, Boutilier K (2014a) The Histone Deacetylase Inhibitor Trichostatin A Promotes Totipotency in the Male Gametophyte. The Plant Cell 26 (1):195-209. doi:10.1105/tpc.113.116491

$\mathrm{L} \mathrm{J,} \mathrm{Jia} \mathrm{H} \mathrm{(2013)} \mathrm{Hydrogen} \mathrm{peroxide} \mathrm{is} \mathrm{involved} \mathrm{in} \mathrm{cGMP} \mathrm{modulating} \mathrm{the} \mathrm{lateral} \mathrm{root}$ development of Arabidopsis thaliana. Plant Signaling \& Behavior 8 (8):e25052. doi:10.4161/psb.25052

Li Q-H, Hong B, Tong Z, Ma C, Guan A-N, Yu J J , Gao J-P (2008) Esta blishment of regeneration system and transformation of $Z \mathrm{~m} 401$ gene in Lilium long iflorum $\times \mathrm{L}$. formosa num. Chinese Joumal of Agricultural Biotechnology 5 (02):113-119. doi:doi:10.1017/S1479236208002222

L SW, Xue LG, Xu SJ , Feng HY, An LZ(2009) Hydrogen peroxide actsas a signal molec ule in the adventitious root formation of mung bean seed lings. Environ Exp Bot 65 (null):63

L W W L Lu H, Cheng Z, Su YH, Han HN, Zhang Y, Zhang XS (2011a) DNA Methylation and Histone Modifications Regulate $\triangleleft$ talic $>$ De Novo $<$ italic $>$ Shoot Regeneration in $<$ italic >Arabidopsis $</$ italic $>$ by Modulating $<$ ita lic $>$ WUSC $\mathrm{HEL}</$ ita lic $>$ Expression and Auxin Signaling. PLoS Genet 7 (8):e1002243. doi:10.1371/joumal.pgen.1002243

L W, Liu H, Cheng Z, Su YH, Han HN, Zhang Y, Zhang XS (2011b) DNA Methylation and Histone Modifications Regulate De Novo Shoot Regeneration in Arabidopsis by Modulating WUSC HEL Expression and Auxin Signaling. PLOSG enetic s 7 (8):e1002243

L X-F, J ia L-Y, Xu J, Deng X-J, Wang Y, Zhang W, Zhang X-P, Fang Q, Zhang D-M, Sun Y, Xu L (2013) FT-Like NFTl Gene May Play a Role in Flower Transition Induced by Heat Accumulation in Narcissus tazetta var. chinensis. Plant and Cell Physiology 54 (2):270281. doi:10.1093/pcp/pcs181

LiX, Qian Q, Fu Z, Wang Y, Xiong G, Zeng D, Wang X, Lu X, Teng S, Hiroshi F, Yuan M, Luo D, Han $B$, J L (2003a) Control of tillering in rice. Nature Reviews Molec ular Cell Biology 422:618621

LiX, Qian Q, Fu Z, Wang Y, Xiong G, Zeng D, Wang X, Liu X, Teng S, Hiroshi F, Yuan M, Luo D, Han B, LJ (2003b) Control of tillering in rice. Nature 422 (6932):618-621

L X, Wang C, Cheng J, Zhang J, da Silva J AT, Lu X, Duan X, L T, Sun H (2014b) Transc riptome analysis of carbohydrate metabolism during bulblet formation and development in Lilium davidii var. unicolor. BMC Plant Biology 14:358. doi:10.1186/s12870-014-0358-4

L Z Trick, H.N. (2005) Rapid method for high-quality RNA isolation from seed endospem conta ining high levels of starch. Biotechniques 38 (6):874-876 
Liang J, Thao L, Challis R, Leyser O (2010) Strigolactone regulation of shoot branching in chrysanthemum (Dendranthema grandiflorum). J Exp Bot 61 (11):3069-3078. doi:10.1093/jxb/erq133

Licausi F, Ohme-Takagi M, Perata P (2013) APETALA2/Ethylene Responsive Factor (AP2/ERF) transc ription factors: mediators of stress responses and developmental programs. New Phytologist 199 (3):639-649. doi:10.1111/nph.12291

Litt A, Kra mer EM (2010) The ABC model and the diversific ation of floral organ identity. Seminars in Cell \& $\quad$ Developmental Biology $21 \quad$ (1):129-137. doi:http://dx.doi.org/10.1016/j.semcdb.2009.11.019

Liu J, Sheng L, Xu Y, L J, Yang Z, Huang H, Xu L (2014) WOX11 and 12 Are Involved in the FirstStep Cell Fate Transition during de Novo Root Organogenesis in Arabidopsis. The Plant Cell Online 26 (3):1081-1093. doi:10.1105/tpc.114.122887

Liu M, Qiao G, Jiang J, Yang H, Xie L, Xie J, Zhuo R (2012) Transcriptome Sequencing and $\langle$ italic $>$ De Novo $<$ italic $>$ Analysis for Ma Bamboo ( $<$ talic $>$ Dendrocalamus latiflorus $<$ /italic > Munro) Using the Illumina Platform. PLoS ONE 7 (10):e46766

Liu W, Wu C, Fu Y, Hu G , Si H, Zhu L, Luan W, He Z, Sun Z(2009) Identific ation and characterization of HTD2: A novel gene negatively regulating tiller bud outgrowth in rice. Planta 230 (4):649-658. doi:10.1007/s00425-009-0975-6

Lodha M, Marco CF, Timmemans MC (2013) The ASYMMEIRIC LEAVES complex maintains repression of KNOX homeobox genes via direct recruitment of Polycomb-repressive complex2. Genes Dev 27 (6):596-601. doi:10.1101/gad.211425.112

Long C, Cheng Z, Wang L, Zuo T (2004) Position effect on the propagation in vitro of different explants from Lilium davidii var. unic olor. Acta Botanica Yunnanic a 26 ((2)):221-225

Long J, Barton MK (2000) Initiation of Axillary and Floral Meristems in Arabidopsis. Developmental Biology 218 (2):341-353. doi:http://dx.doi.org/10.1006/dbio.1999.9572

Lorenz MGO, Cortes LM, Lorenz JJ, Liu ET (2003) Strategy for the Design of Custom cDNA Mic roa rrays. BioTechniques 34:1264-1270

Lu G, Zou Q, Guo D, Zhuang X, Yu X, Xiang X, Cao J (2007) Agrobacterium tumefaciensmediated transformation of Narcissus tazzeta var. chinensis (Plant Cell Reports). 26 (9):1585-1593. doi:10.1007/s00299-007-0382-z

Lu R, Martin-Hemandez AM, Peart JR, Malc uit I, Baulcombe DC (2003) Virus-induced gene silencing in plants (RNA interference). Methods 30 (4):296-303. doi:http://dx.doi.org/10.1016/S1046-2023(03)00037-9

Lu Y, Huggins P, Bar-J oseph Z (2009) Cross species a nalysis of mic roa ray expression data. Bio informatic s 25 (12):1476-1483. doi:10.1093/bioinfo matic s/btp247

Maere S, Heymans K, Kuiper M (2005) BiNG O: a Cytosc a pe plugin to assess overrepresentation of Gene Ontology categories in Biologic al Networks. Bio informatic s 21 (16):3448-3449 
Maia J, Dekkers BJ W, Provart NJ, Ligterink W, Hilhorst HWM (2011) The Re-Establishment of Desic cation Tolerance in Geminated $<$ ita lic >Arabidopsis tha lia na </italic > Seeds and Its Associated Transc riptome. PLoS ONE 6 (12):e29123

Malik M, Bach A (2010) Somatic embryogenesis induction and morphogenesis direction in na rcissus culture (Narcissus L.) of 'Cartton' depending on the initial explants type. Post Nauk Rol 551:175-181

Manassero Nora GU, Viola Ivana L, Welchen E, Gonzalez Daniel H (2013) TCP transcription factors: architectures of plant form. BioMolecular Concepts, vol 4. doi:10.1515/bmc2012-0051

Ma o J -L, Mia o Z-Q, Wang Z, Yu L-H, Ca X-T, Xiang C-B (2016) Arabidopsis ERF1 Mediates CrossTalk between Ethylene and Auxin Biosynthesis during Primary Root Elongation by Regulating ASA1 Expression. PLOS Genetics 12 (1):e1005760

Marguerat S, BählerJ (2010) RNA-seq: from technology to biology. Cellular and Molecular Life Sciences 67 (4):569-579. doi:10.1007/s00018-009-0180-6

Marhavý P, Vanstraelen M, De Rybel B, Zhaojun D, Bennett MJ, Beeckman T, Benková E (2012) Auxin reflux between the endodemis and pericycle promotes lateral root initiation. The EMBO J oumal 32 (1):149-158

Marinangeli PA, Hemandez LF, Pellegrini CP, Curvetto NR, (2003) Bulblet differentiation after scale propagation of Lilium longiflorum

J oumal of the Americ an Soc iety for Hortic ultural Science 128 (3):324-329

Martín-Trillo M, Cubas P (2010) TCP genes: a family snapshot ten years later. Trends in Plant Science 15 (1):31-39. doi:http://dx.doi.org/10.1016/j.tplants.2009.11.003

Martín-Trillo M, Grandío EG, Serra F, Marcel F, Rodríguez-Buey ML, Schmitz G, Theres K, Bendahmane A, Dopazo H, Cubas P (2011) Role of tomato BRANCHED1-like genes in the control of shoot branching. The Plant Joumal 67 (4):701-714. doi:10.1111/j.1365313X.2011.04629.x

Martin J A, Wang Z(2011) Next-generation transc riptome a ssembly. 12 (10):671-682

Maślanka M, Bach A (2014) Induction of bulb organogenesis in in vitro cultures of tarda tulip (Tulipa tarda Stapf.) from seed-derived explants. In Vitro Cellular \& Developmental Biology - Plant 50 (6):712-721. doi:10.1007/s11627-014-9641-1

Mason MG, Ross J , Babst BA, Wienclaw BN, Beveridge CA (2014) Sugar demand, not a uxin, is the initial regulator of apical dominance. Proceedings of the National Academy of Sciences 111 (16):6092-6097. doi:10.1073/pnas.1322045111

Matsuo N, Makino M, Banno H (2011) Ara bid opsis ENHANCER OF SHOOTREG ENERATION (ESR)1 and ESR2 regulate in vitro shoot regeneration and their expressions are differentially regulated. Plant Sc i 181 (1):39-46. doi:10.1016/j.plantsc i.2011.03.007

McCouch SR (2001) Genomics and Synteny. Plant Physiology 125 (1):152-155. doi:10.1104/pp.125.1.152 
Meister G (2013) Argona ute proteins: functional insights and emerging roles. 14 (7):447-459 Miller GAD, Mittler RON (2006) Could Heat Shock Transcription Factors Function as Hydrogen Peroxide Sensors in Plants? Annals of Botany 98 (2):279-288. doi:10.1093/a ob/mcl107 Minas GJ (2007) In vitro propagation of Akama tulip via Adventitious Organogenesis from Bulb Slices. Acta Hortic ulturae, vol 755.

Ming R, VanBuren R, Wai CM, Tang H, Schatz MC , BowersJ E, LyonsE, Wang M-L, Chen J , Biggers E, Zhang J, Huang L, Zhang L, Mia o W, Zhang J, Ye Z, Mia o C, Lin Z, Wang H, Zhou H, Yim WC, Priest HD, Zheng C, Woodhouse M, Edger PP, Guyot R, Guo H-B, Guo H, Zheng G, Singh R, Sharma A, Min X, Zheng Y, Lee H, Gurtowski J, Sedlazeck FJ, Harkess A, McKa in MR, Liao Z, Fang J, Liu J , Zhang X, Zhang Q, Hu W, Q in Y, Wang K, Chen L-Y, Shirley N, Lin Y-R, Liu L-Y, Hemandez AG, Wright CL, Bulone V, Tuskan GA, Heath K, Zee F, Moore PH, Sunkar R, Leebens-Mack J H, Mockler T, Bennetzen J L, Freeling M, Sankoff D, Paterson AH, Zhu X, Yang X, Smith JAC, Cushman JC, Paull RE, Yu Q (2015) The pineapple genome and the evolution of CAM photosynthesis. advance online publication

Minibayeva F, Beckett RP, Krannerl (2015) Roles of apoplastic peroxidases in plant response to wounding. Phytochemistry 112:122-129. doi:https://doi.org/10.1016/j.phytochem.2014.06.008

Miyawaki K, Matsumoto-Kitano M, Kakimoto T (2004) Expression of cytokinin biosynthetic isopentenyltransferase genes in Arabidopsis: tissue spec ific ity and regulation by auxin, cytokinin, and nitrate. Plant J 37 (1):128-138

Mondragón-Palomino M, Trontin C (2011) High time for a roll call: gene duplication and phylogenetic relationships of TCP-like genes in monoc ots. Annals of Bota ny 107 (9):15331544. doi:10.1093/aob/mcr059

Moore S, Payton P, Wright M, Tanksley S, Giovannoni J (2005) Utilization of tomato mic roa rays for comparative gene expression analysis in the Solanaceae. J Exp Bot 56 (421):28852895. doi:10.1093/jxb/eri283

Morelli G, Ruberti I (2002) Light and shade in the photocontrol of <em>Arabidopsis $</ e m>$ growth. Trends in Plant Science 7 (9):399-404. doi:10.1016/s1360-1385(02)02314-2

Moreno-Pachon MN, Leeggangers HACF, Nijveen H, Severing E, Hilhorst H, Immink RG H (2016) Elucidating and mining the Tulipa and Lilium transcriptomes. Plant Mol Biol:1-13. doi:10.1007/s11103-016-0508-1

Moreno-Risueno MA, Sozzani R, Yardımcı GG, Petricka JJ, Vernoux T, Blilou I, Alonso J, Winter CM, Ohler U, Scheres B, Benfey PN (2015) Transcriptional control of tissue formation throughout root development. Science 350 (6259):426-430.

Momis SE, Tumbull CG N, Murfet IC , Beveridge CA (2001) Mutational a na lysis of branching in pea. Evidence that Rms1 and Rms5 regulate the same novel signal. Plant Physiology 126 (3):1205-1213 
Motte H, Vereecke D, Geelen D, Werbrouck S (2014) The molecular path to in vitro shoot regeneration. Biotechnology Advances $32 \quad$ (1):107-121. doi:http://doi.org/10.1016/j.biotechadv.2013.12.002

Motte H, Verstraeten I, WerbrouckS, Geelen D (2011) CUC 2 as an early markerfor regeneration competence in Arabidopsis root explants. Joumal of Plant Physiology 168 (13):15981601. doi:http://dx.doi.org/10.1016/j.jplph.2011.02.014

Müller D, Schmitz G, Theres K (2006) Blind homologous R2R3 Myb genes control the pattem of lateral menistem initiation in Arabidopsis. Plant Cell 18 (3):586-597. doi:10.1105/tpc. 105.038745

Nagarajan N, Pop M (2013) Sequence assembly demystified. 14 (3):157-167

Nakano T, Suzuki K, Fujimura T, Shinshi H (2006) Genome-Wide Analysis of the ERF Gene Family in Arabidopsis and Rice. Plant Physiology 140 (2):411-432. doi:10.1104/pp.105.073783

Napoli C (1996) Highly branched phenotype of the petunia dad1-1 mutant is reversed by grafting. Plant Physiology 111 (1):27-37

Napoli CA, Beveridge CA, Snowden KC (1999) Reevaluating concepts of apical dominance and the control of axillary bud outgrowth. Current topics in developmental biology 44:127-169

Narita NN, Moore S, Horiguchi G, Kubo M, Demura T, Fukuda H, Goodrich J, Tsukaya H (2004) Overexpression of a novel small peptide ROTUNDIFOLA4 decreases cell proliferation and alters leaf shape in Arabidopsis thaliana. The Plant Joumal 38 (4):699-713. doi:10.1111/j.1365-313X.2004.02078.x

Neale D, Wegrzyn J, Stevens K, Zmin A, Puiu D, Crepeau M, Cardeno C, Koriabine M, HoltzMoris A, Liechty J, Martinez-Garcia P, Vasquez-Gross H, Lin B, Zeve J, Dougherty W, Fuentes-Soriano S, Wu L-S, Gilbert D, Marc a is G, Roberts M, Holt C, Yandell M, Davis J, Smith K, Dean J, Lorenz W, Whetten R, Sederoff R, Wheeler N, McGuire P, Main D, Loopstra C, Mockaitis K, deJ ong P, Yorke J, Salzberg S, Langley C (2014) Decoding the massive genome of loblolly pine using haploid DNA and novel assembly strategies. Genome Biology 15 (3):R59

Neelakandan AK, Wang K (2012) Recent progress in the understanding of tissue cultureinduced genome level changes in plants and potential a pplic ations. Plant Cell Rep 31 (4):597-620. doi:10.1007/s00299-011-1202-z

Nic olas M, Cubas P (2016) Chapter 16 - The Role of TCP Transcription Factors in Shaping Flower Structure, Leaf Morphology, and Plant Architecture A2 - Gonzalez, Daniel H. In: Plant Transcription Factors. Academic Press, Boston, pp 249-267. doi:http://dx.doi.org/10.1016/B978-0-12-800854-6.00016-6

Nic olas M, Rodríguez-Buey Mańa L, Franco-Zomilla J osé M, Cubas P (2015) A Recently Evolved Altemative Splice Site in the BRANCHEDla Gene Controls Potato Plant Architecture. Current Biology 25 (14):1799-1809. doi:http://dx.doi.org/10.1016/j.cub.2015.05.053 
Nishiuchi Y, Koster J (1988) Difference in organogenic activity in the scale tissue culture between tulip bulbsgrown at Asahikawa and bulbs grown in the Netherlans. J oumal of Hokka ido University, faculty of Education (II B) 39:1-6

Novak O, Hauserova E, Amakorova P, Dolezal K, Stmad M (2008) Cytokinin profiling in plant tissues using ultra-performance liquid chromatography-electrospray tandem mass spec trometry. Phytochemistry 69 (11):2214-2224. doi:10.1016/j.phytochem.2008.04.022

Novak O, Henykova E, Sa iranen I, Kowalc zyk M, Pospisil T, Ljung K (2012) Tissue-spec ific profiling of the Arabidopsis thaliana auxin metabolome. Plant J 72 (3):523-536. doi:10.1111/j.1365-313X.2012.05085.x

Núñez de Cáceres FF, Davey MR, Wilson ZA (2011) A Rapid and Efficient AgrobacteriumMediated Transfomation Protocol for Lilium. Acta horticulture 900:161-167. doi:10.17660/Acta Hortic .2011.900.19

Oikawa T, Kyozuka J (2009) Two-Step Regulation of LAX PANICLE1 Protein Accumulation in Axillary Meristem Formation in Rice. The Plant Cell Online 21 (4):1095-1108. doi:10.1105/tpc.108.065425

O kubo H (2012) Domancy. In: Kamenetsky R, Okubo H (eds) Omamental Geophytes: From Basic Science to Sustainable Production. CRC Press, pp 233-260. doi:doi:10.1201/b12881-10

Okubo H, Sochacki D (2012) Botanical and Horticultural Aspects of Mayor Omamental Geophytes. In: Omamental Geophytes. CRC Press, pp 77-122. doi:doi:10.1201/b128811

Parra G, Bradnam K, Korf I (2007) CEG MA: a pipeline to accurately annotate core genes in $\begin{array}{llll}\text { eukaryotic genomes. } & \text { Bioinformatics } 23 & \text { (9):1061-1067. }\end{array}$ doi:10.1093/bioinformatic s/btm071

Pasare SA, Ducreux L M, Momis WL, Campbell R, Shama SK, Roumeliotis E, Kohlen W, van der Krol S, Bramley PM, Roberts AG, Fraser PD, Taylor MA (2013) The role of the potato (Solanum tuberosum) CCD8 gene in stolon and tuber development. New Phytologist 198 (4):1108-1120. doi:10.1111/nph.12217

Petrasek J, Mravec J, Bouchard R, Blakeslee J J, Abas M, Seifertova D, Wisniewska J, Tadele Z, Kubes M, Covanova M, Dhonukshe P, Skupa P, Benkova E, Perry L, Krecek P, Lee OR, Fink GR, Geisler M, Murphy AS, Luschnig C, Zazimalova E, Friml J (2006) PIN proteins perform a rate-limiting function in cellular auxin efflux. Science 312 (5775):914-918. doi:10.1126/science. 1123542

Pi L, Aichinger E, van der Graaff E, Lavata-Penis Cristina I, Weijers D, Hennig L, Groot E, Laux T (2015) Organizer-Denived WOX5 Signal Maintains Root Columella Stem Cells through Chromatin-Mediated Repression of CDF4 Expression. Developmental Cell 33 (5):576588. doi:https://doi.org/10.1016/j.devcel.2015.04.024 
Podwyszyńska M (2005) Somaclonal variation in mic ropropagated tulips based on phenotype observation. J oumal of fruit and omamental plant research 13:109-122

Podwyszyńska M (2006) Effect of ethylene, auxine and methyl jasmonate on bulb formation in vitro in tulip shoot cultures. Zeszyty Problemowe Postepow Nauk Rolnic zych 510:461-469

Podwyszynska M, Sochacki D (2010) Mic ropropagation of tulip: production of virus-free stock plants. Methods Mol Biol 589:243-256. doi:10.1007/978-1-60327-114-1_23

Poza-Camión C, Aguilar-Martínez JA, Cubas P (2007) Role of TCP Gene BRANCHED1 in the Control of Shoot Branching in Arabidopsis. Plant Signaling \& Behavior 2 (6):551-552. doi:10.4161/psb.2.6.4811

Rameau C (2010) Strigolactones, a novel class of plant homone controlling shoot branching. Comptes Rendus - Biologies 333 (4):344-349

Rameau C, Bertheloot J, Leduc N, Andrieu B, Foucher F, Sakr S (2015) Multiple pathways regulate shoot branching. Frontiers in Plant Science 5 (741). doi:10.3389/fpls.2014.00741 Rameau C, Pillot JP (2010) Strigolac tone effect in shoot branching. Acta Horticulturae, vol 884. Rebers M, Vermeer E, Knegt E, Shelton CJ, van der Plas LHW (1994) Gibberellins in tulip bulb $\begin{array}{lllll}\text { sprouts during } & \text { storage. } & \text { Phytochemistry } 269-272 .\end{array}$ doi:http://dx.doi.org/10.1016/S0031-9422(00)97059-6

Reddy SK, Holalu SV, Casal JJ, Finlayson SA (2013) Abscisic Acid Regulates Axillary Bud Outgrowth Responsesto the Ratio of Red to Far-Red Light. Plant Physiology 163 (2):10471058. doi:10.1104/pp.113.221895

Rees AR (1966) The Physiology of O mamental Bulbous Plants. Botanical Review 32 (1):1-23. doi:10.2307/4353723

Rees AR (1968) The Initiation and Growth of Tulip Bulbs. Anna Is of Botany 32 (1):69-77

Rees AR (1981) Concepts of domancy as illustrated by the tulip and other bulbs. Annals of Applied Biology 98 (3):544-548. doi:10.1111/j.1744-7348.1981.tb00789.x

Rees AR (1992) Oma mental bulbs, coms and tubers. CAB Intemational, Wallingford

Rice RD, Alderson PG, Wright NA (1983) INDUCTION OF BULBING OF TUUP SHOOTS IN VITRO. Scientia Hortic ulturae 20:377--390

Riec hmann J L, Heard J, Martin G, Reuber L, J iang C-Z, Keddie J , Adam L, Pineda O, Ratc liffe OJ , Sama ha RR, Creelman R, Pilgrim M, Broun P, Zhang J Z, G handehari D, Sheman BK, -L. Yu G (2000) Arabidopsis Transcription Factors: Genome-Wide Comparative Analysis Among Eukaryotes. Science 290 (5499):2105-2110. doi:10.1126/science.290.5499.2105

Riesgo A, Andrade SC S, Sha ma PP, Novo M, Pérez-Porro AR, Va htera V, Gonzá lezVL, Kawa uc hi GY, Giribet G (2012) Comparative description of ten transcriptomes of newly sequenced invertebrates and effic iency estimation of genomic sampling in non-model taxa. Frontiers in Zoology 9:33-33. doi:10.1186/1742-9994-9-33 
Rietveld PL, Wilkinson C, Franssen HM, Balk PA, Plas LHWvd, Weisbeek PJ , Boer ADd (2000) Low temperature sensing in tulip (tulipa gesneriana L) is mediated through an increased response to a uxin. J oumal of Experimental Botany 51 (2000):587-594

Rijpkema AS, Vandenbussc he M, Koes R, Heijmans K, Gerats T(2010) Variations on a theme: Changes in the floral ABCs in angiospems. Seminars in Cell \& Developmental Biology 21 (1):100-107. doi:http://dx.doi.org/10.1016/j.semc db.2009.11.002

Rinne P, Welling A, Vahala J, Ripel L, Ruonala R, Kangasjarvi J, van der Schoot C (2011) Chilling of domant buds hyperinduces FLOWERING LOCUS T and recruits GA-inducible 1,3beta-gluc a nasesto reopen signal conduits and release domancy in Populus. Plant Cell 23:130 - 146

Rinne PLH, Kaikuranta PM, Van Der Schoot C (2001) The shoot apical menistem restores its symplasmic organization during chilling-induced release from domancy. The Plant J oumal 26 (3):249-264. doi:10.1046/j.1365-313X.2001.01022.x

Rinne PLH, Paul LK, Vahala J , Kangasjärvi J, van der Schoot C (2016) Axillary buds are dwa rfed shoots that tightly regulate GA pathway and GA-inducible 1,3- $\beta$-glucanase genes during branching in hybrid aspen. J Exp Bot 67 (21):5975-5991. doi:10.1093/jxb/erw352

Robinson MD, McCarthy DJ , Smyth GK (2009) edgeR: a Bioconductor package for differential expression a na lysis of digital gene expression data. Bio informatic s 26 (1):139-140

Rohde A, Bhalera RP (2007) Plant domancy in the perennial context. Trends in Plant Science 12 (5):217-223. doi:http://dx.doi.org/10.1016/j.tplants.2007.03.012

Rossmann S, Kohlen W, Hasson A, Theres K (2015) Lateral suppressor and Goblet act in hierarchical order to regulate ectopic menistem formation at the base of tomato leaflets. Plant J 81 (6):837-848. doi:10.1111/tpj.12782

Rosspopoff O, Chelysheva L, Saffar J, Lecorgne L, Gey D, Caillieux E, Colot V, Roudier F, Hilson P, Berthomé R, Da Costa M, Rech P (2017) Direct conversion of root primordium into shoot meristem relies on timing of stem cell niche development. Development 144 (7):1187-1200. doi:10.1242/dev.142570

Rost TL, Paterson K (1976) The Developmental Anatomy of Adventive Plantlets from Leavesand Leaf Segments of Crassula a rgentea (Crassula ceae). Bota nic al G a zette 137 (3):203-210 Roumeliotis E, Kloosterman B, O ortwijn M, Kohlen W, Bouwmeester HJ , Visser RG F, Ba chem CWB (2012) The effec ts of a uxin and strig olactones on tuber initia tion and stolon a rchitecture in potato. J Exp Bot 63 (12):4539-4547. doi:10.1093/jxb/ers132

Sa batini S, Beis D, Wolkenfelt H, Murfett J, Guilfoyle T, Malamy J, Benfey P, Leyser O, Bechtold N, Weisbeek P, Scheres B (1999) An auxin-dependent distal organizer of pattem and polarity in the Arabidopsis root. Cell 99 (5):463-472

Sa batini S, Heidstra R, Wild water M, Scheres B (2003) SCARECROW is involved in positioning the stem cell niche in the Arabidopsis root menistem. Genes \& Development 17 (3):354-358. doi:10.1101/gad.252503 
Saito K, Matsuda F (2010) Metabolomics for Functional Genomics, Systems Biology, and Biotechnology. Annual Review of Plant Biology 61 (1):463-489. doi:doi:10.1146/annurev.amplant.043008.092035

Salentijn El, Pereira A, Angenent G, Linden CG, Krens F, Smulders MM, Vosman B (2007) Plant translational genomic s: from model species to crops (Molec ular Breeding). 20 (1):1-13. doi:10.1007/s11032-006-9069-3

Sa lic A, Mitchison T (2008) A chemic al method forfast and sensitive detection of DNA synthesis in vivo. Proceedings of the National Academy of Sciences 105 (7):2415-2420. doi:10.1073/pnas.0712168105

Sa niewski M, Ka wa-Miszc za k L, Wegrzynowic z-LesiakE, O kubo H (2000) Role of ABA, gib berellins and auxin in domancy and domancy release of tulip bulbs. In. CABI Publishing, Wallingford, pp 227-243. doi:10.1079/9780851994475.0227

Schatz MC, Delcher AL, Salzberg SL (2010) Assembly of large genomes using secondgeneration sequencing. Genome Research 20 (9):1165-1173. doi:10.1101/gr.101360.109

Schmid M, Davison TS, Henz SR, Pape UJ , DemarM, Vingron M, Scholkopf B, Weigel D, Lohmann JU (2005) A gene expression map of Arabidopsis thaliana development. 37 (5):501-506 Schmitz G, Theres K (2005) Shoot and inflorescence branching (Cell signalling and gene regulation). Current Opinion in Plant Biology 8 (5):506-511. doi:http://dx.doi.org/10.1016/j.pbi.2005.07.010

Schnable PS, Ware D, Fulton RS, Stein J C, Wei F, Pastemak S, Liang C, Zhang J , Fulton L, Graves TA, Minx P, Reily AD, Courtney L, Kruchowski SS, Tomlinson C, Strong C, Delehaunty K, Fronick C, Courtney B, Rock SM, Belter E, Du F, Kim K, Abbott RM, Cotton M, Levy A, Marchetto P, Ochoa K, Jackson SM, Gillam B, Chen W, Yan L, Higginbotham J, Cardenas M, Waligorski J, Applebaum E, PhelpsL, Falc one J, Kanchi K, Thane T, Sc imone A, Thane N, Henke J, Wang T, Ruppert J, Shah N, Rotter K, Hodges J, Ingenthron E, Cordes M, Kohlberg S, Sgro J, Delgado B, Mead K, Chinwalla A, Leonard S, Crouse K, Collura K, Kudma D, Cumie J , He R, Angelova A, Rajasekar S, Mueller T, Lomeli R, Scara G, Ko A, Delaney K, Wissotski M, Lopez G, Campos D, Braidotti M, Ashley E, Golser W, Kim H, Lee S, Lin J, Dujmic Z, Kim W, Talag J , Zuccolo A, Fan C, Sebastian A, Kramer M, Spiegel L, Nascimento L, Zutavem T, Miller B, Ambroise C, Muller S, Spooner W, Narechania A, Ren L, Wei S, Kumari S, Faga B, Levy MJ, McMahan L, Van Buren P, Vaughn MW, Ying K, Yeh C-T, Emrich SJ , J ia Y, Kalyanaraman A, Hsia A-P, Barbazuk WB, Baucom RS, Brutnell TP, Ca pita NC, Chapa rro C, Chia J-M, Deragon J-M, Estill J C, Fu Y, Jeddeloh JA, Han Y, Lee H, Li P, Lisch DR, Lu S, Liu Z, Nagel DH, McCann MC, SanMiguel P, Myers AM, Nettleton D, Nguyen J , Penning BW, Ponnala L, Sc hneider KL, Schwartz DC, Sharma A, Soderlund C, Springer NM, Sun Q, Wang H, Waterman M, Westerman R, Wolfgruber TK, Yang L, Yu Y, Zhang L, Zhou S, Zhu Q, Bennetzen J L, Dawe RK, J iang J, J iang N, Presting GG, Wessler SR, Aluru S, Martienssen RA, Clifton SW, McCombie WR, 
Wing RA, Wilson RK (2009) The B73 Ma ize Genome: Complexity, Diversity, and Dynamics. Science 326 (5956):1112-1115. doi:10.1126/science.1178534

Schneeberger K, Weigel D (2011) Fast-forward genetics enabled by new sequencing technologies. Trends in Plant Science 16 (5):282-288. doi:http://dx.doi.org/10.1016/j.tplants.2011.02.006

Schraud olf H, ReinertJ (1959) Interaction of Plant Growth Regula tors in Regeneration Proc esses. 184 (4684):465-466

Schumacher K, Schmitt T, Rossberg M, Schmitz G, Theres K (1999) The Lateral suppressor (LS) gene of tomato encodes a new member of the VHIID protein family. Proceedings of the National Aca demy of Sciences 96 (1):290-295. doi:10.1073/pnas.96.1.290

Seale M, Bennett T, Leyser $O(2017)<e m>B R C 1</$ em $>$ expression regulates bud activation potential, but is not necessary or sufficient for bud growth inhibition in Arabidopsis. Development. doi:10.1242/dev.145649

Sena G, Wang X, Liu H-Y, Hofhuis H, Bimbaum KD (2009) Organ regeneration does not require a functional stem cell nic he in plants. 457 (7233):1150-1153

Sequencing Project Intemational Rice G (2005) The map-based sequence of the rice genome. 436 (7052):793-800

Sequencing ProjectIntemational Rice G (2005) The map-based sequence of the rice genome. 436 (7052):793-800

Shahin A, van Kaauwen M, Esselink D, Bargsten J, van Tuyl J, Visser RG, Arens P (2012a) Generation and analysis of expressed sequence tags in the extreme large genomes Lilium and Tulipa. BMC Genomic s 13 (1):640

Shahin A, van Kaauwen M, Esselink D, Bargsten J W, van Tuyl J M, Visser RG F, Arens P (2012b) Generation and analysis of expressed sequence tags in the extreme large genomes Lilium and Tulipa. BMC Genomic s:640. doi:10.1186/1471-2164-13-640

Shimizu-Sato S, Mori H (2001) Control of outgrowth and domancy in axillary buds. Plant Physiology 127 (4):1405-1413. doi:10.1104/pp.127.4.1405

Skoog F, Miller CO (1957) Chemical regulation of growth and organ formation in plant tissues c ultured in vitro. Symposia of the Society for Experimental Biology 11:118-130

Slater A, Scott NW, Fowler MR (2003) Plant biotechnology : the genetic manipulation of plants. Oxford University Press, Oxford

Smoot ME, O no K, RuscheinskiJ, Wang P-L, IdekerT(2011) Cytosc a pe 2.8: new featuresfor data integration and network visualization. Bioinformatics 27 (3):431-432. doi:10.1093/bio info matic s/ btq 675

Spicer R, Tisdale-Orr T, Talavera C (2013) Auxin-Responsive DR5 Promoter Coupled with Transport Assa ys Suggest Separate but Linked Routes of Auxin Transport during Woody Stem Development in Populus. PLOSO NE 8 (8):e72499 
Srikanth A, Schmid M (2011) Regulation of flowering time: all roads lead to Rome (Cellular and Molecular Life Sc iences). 68 (12):2013-2037. doi:10.1007/s00018-011-0673-y

Steeves TA, Sussex IM (1972) Pattems in Plant Development. Prentice-Hall,

Strable J, Borsuk L, Nettleton D, Schnable PS, Irish EE (2008) Mic roa rray a nalysis of vegeta tive phase change in maize. The Plant Joumal 56 (6):1045-1057. doi:10.1111/j.1365313X.2008.03661.x

Sugimoto K, Gordon SP, Meyerowitz EM (2011) Regeneration in plants and animals: dedifferentiation, transdifferentiation, or just differentiation? Trends Cell Biol 21 (4):212218. doi:10.1016/j.tcb.2010.12.004

Sugimoto K, J iao Y, Meyerowitz EM (2010) Arabidopsis Regeneration from Multiple Tissues Occurs via a Root Development Pathway. Developmental Cell 18 (3):463-471. doi:http://dx.doi.org/10.1016/j.devcel.2010.02.004

Suh J K, Roh MS (2014) New technique for cut flower production from bulbils of the Asia tic hybrid lily (Liliumxelegans Thunb.). Scientia Hortic ulturae 165:374-383. doi:https://doi.org/10.1016/j.sc ienta.2013.11.012

Sun C, Sathish P, Ahland sberg S, J a nsson C (1999) Ana lyses of isoa myla se gene activity in wildtype barley indicate its involvement in starch synthesis. Plant Mol Biol 40 (3):431-443. doi:10.1023/a:1006217506090

Supek F, Bošnjak M, Škunca N, Šmuc T (2011) REVIGO Summarizes and Visualizes Long Lists of Gene Ontology Terms. PLOSONE 6 (7):e21800

Svacinova J, Novak O, Plackova L, Lenobel R, Holik J, Stmad M, Dolezal K (2012) A new approach for cytokinin isolation from Arabidopsis tissues using miniaturized purific ation: pipette tip solid-phase extraction. Plant methods 8 (1):17. doi:10.1186/1746-4811-8-17

Taeb AG, Alderson PG (1990a) Effect of low temperature and sucrose on bulb development and on the carbohydrate status of bulbing shoots of tulip in vitro. The Joumal of hortic ultural sc ience 65 (2):193-197

Taeb AG, Alderson PG (1990b) Shoot production and bulbing of tulip in vitro related to ethylene. The J oumal of hortic ultural science 65 (2):199-204

Takada S, Hibara K, Ishida T, Tasaka M (2001) The CUP-SHAPED COTYLEDON1 gene of Arabid opsis regulates shoot a pical meristem formation. Development 128 (7):1127-1135

Takeda T, Suwa Y, Suzuki M, Kitano H, Ueguchi-Tanaka M, Ashikari M, Matsuoka M, Ueguchi C (2003) The OsTB1 gene negatively regulates lateral branching in rice. The Plant J oumal 33 (3):513-520. doi:10.1046/j.1365-313X.2003.01648.x

Tanaka M, Kikuchi A, Kama da H (2008) The Ara bid opsis Histone Deacetyla ses HDA6 and HDA19 Contribute to the Repression of Embryonic Properties after Gemination. Plant Physiology 146 (1):149-161. doi:10.1104/pp.107.111674 
Tanaka N, Itoh H, Sentoku N, Kojima M, Sakakibara H, Izawa T, Itoh J -I, Nagato Y (2011) The COP1 Ortholog PPS Regulates the J uvenile-Adult and Vegetative-Reproductive Phase Changes in Rice. The Plant Cell Online 23 (6):2143-2154. doi:10.1105/tpc.111.083436

Tang N, van der Lee T, Shahin A, Hold inga M, Bijman P, Caser M, Visser RG, van Tuyl J M, Arens P (2015) Genetic mapping of resistance to Fusarium oxysporum f. sp. tulipae in tulip. Molecular Breeding 35 (5):122

Tasaki K, Terada H, Masuta C, Yamagishi M (2016) Virus-induced gene silencing (VIGS) in $<>>$ ilium leichtlinii $</ \mathrm{i}>$ using the $\langle>C$ Cucumber mosaic vinus $</>$ vector. Plant Biotec hnology 33 (5):373-381. doi:10.5511/plantbiotec hnology.16.1018a

Treangen T, Salzberg SL (2012) Repetitive DNA and next-generation sequencing: computational challenges and solutions. 13 (1):36-46

Trevaskis B, Tadege M, Hemming MN, Peacock WJ, Dennis ES, Sheldon C (2007) Short Vegetative Phase-Like MADS-Box Genes Inhibit Floral Meristem Identity in Barley. Plant Physiology 143 (1):225-235. doi:10.1104/pp.106.090860

Tsaftaris A, Pasentsis K, Kalivas A, Michailid ou S, Madesis P, Argiriou A (2012) Isolation of a CENTRORADIALS/TERMINAL FLOWER1 homolog in saffron (Crocus sativus L): characterization and expression analysis (Molecular Biology Reports). 39 (8):7899-7910. doi:10.1007/s11033-012-1634-8

Tsaftaris A, Pasentsis K, Makris A, Darzentas N, Polidoros A, Ka livas A, Argiriou A (2011) The study of the E-class SEPAШATA3-like MADS-box genes in wild-type and mutant flowers of cultiva ted saffron croc us (Crocus sativus L.) and itsputative progenitors. J oumal of Plant Physiology 168 (14):1675-1684. doi:http:// dx.doi.org/10.1016/i.jplph.2011.03.015

Tsaftaris AS, Pa sentsis K, lliopoulos I, Polidoros AN (2004) Isolation of three homolog ous AP1-like MADS-box genes in croc us (C rocus sativus $L$.) and characterization of their expression. Plant science : an intemational joumal of experimental plant biology 166 (5):1235-1243

Tsukagoshi H, Busch W, Benfey PN (2010) Transcriptional Regulation of ROS Controls Transition from Proliferation to Differentiation in the Root. Cell 143 (4):606-616. doi:http://dx.doi.org/10.1016/j.cell.2010.10.020

Tuskan GA, DiFazio S, Jansson S, Bohlmann J, Grigoriev I, Hellsten U, Putnam N, Ralph S, Rombauts S, Salamov A, Schein J, Sterck L, Aerts A, Bhalera o RR, Bhalerao RP, Blaudez D, Boerjan W, Brun A, Brunner A, Busov V, Campbell M, Carlson J , Chalot M, Chapman J, Chen GL, Cooper D, Coutinho PM, Couturier J, Covert S, Cronk Q, Cunningham R, Davis J, Degroeve S, xe, jardin A, dePamphilis C, Detter J, Dirks B, Dubchak I, Duplessis S, Ehlting J , Ellis B, Gendler K, Goodstein D, G ribskov M, G rimwood J , G roover A, Gunter L, Ha mberger B, Heinze B, Helariutta Y, Henrissat B, Holligan D, Holt R, Huang W, IslamFa ridi N, J ones S, J ones-Rhoa des M, J orgensen R, J oshi C, Kangasj, xe, rvi J , Ka rlsson J, Kelleher C, Kirkpatrick R, Kirst M, Kohler A, Kalluri U, La rimerF, Leebens-MackJ, Lepl, xe, C. J , Locascio P, Lou Y, Lucas S, Martin F, Montanini B, Napoli C, Nelson DR, Nelson C, 
Nieminen K, Nilsson O, Pereda V, Peter G, Philippe R, Pila te G, Poliakov A, Razumovskaya J, Richardson P, Rinaldi C, Ritland K, Rouz, xe, P., Ryaboy D, Schmutz J, Schrader J, Segeman B, Shin H, Siddiqui A, Sterky F, Temy A, Tsai CJ, Uberbacher E, Unneberg P, Vahala J, Wall K, Wessler S, Yang G, Yin T, Douglas C, Marra M, Sandberg G, Van de PeerY, RokhsarD (2006) The Genome of Black Cottonwood, Populus trichoc arpa (Torr. \& Gray). Science 313 (5793):1596-1604

Tzeng T-Y, Yang C-H (2001) A MADS Box Gene from Lily (Lilium Longiflorum) is Suffic ient to Generate Dominant Negative Mutation by Interacting with PISTLATA (PI) in Arabidopsis thaliana. Plant and Cell Physiology 42 (10):1156-1168. doi:10.1093/pcp/pce151

Van Aartrijk J, Blom-Bamhoom GJ (1981) Growth regulator requirements for adventitious regeneration from Lilium bulb-scale tissue in vitro, in relation to duration of bulb storage and cultivar. Sc ientia Hortic ulturae 14 (3):261-268. doi:http://dx.doi.org/10.1016/03044238(81)90021-2

van Aartrijk J , Blom-Bamhoom GJ (1983) Adventitious Bud Formation From Bulb-scale Expiants of Lilium speciosum Thunb. in vitro. Effects of Wounding, TIBA, and Temperature. Zeitschnift für Pflanzenphysiologie 110 (4):355-363. doi:http://dx.doi.org/10.1016/50044$\underline{328 \times(83) 80185-8}$

Van Aartrijk J , Blom-Bamhoom GJ (1984) Adventitious Bud Formation from Bulb-scale Explants of Lilium speciosum Thunb. in vitro Interacting effects of NAA, TBA, wounding, and $\begin{array}{lllll}\text { temperature. Joumal of Plant Physiology } 116 & \text { (5):409-416. }\end{array}$ doi:http://dx.doi.org/10.1016/S0176-1617(84)80132-7

Van Aartrijk J , Blom-Bamhoom GJ , Bruinsma J (1986) 4 - A ROLE FOR ETHYLENE BIOSYNTHESIS IN ADVENTTIOOUS BUD FORMATION ON BULB-SCALE TISSUE OF LUUM SPECIOSUM. In: Plant Tissue Culture and its Agricultural Applications. Butterworth-Heinemann, pp 55-61. doi:http://dx.doi.org/10.1016/B978-0-407-00921-9.50010-5

Van Emmenes L, Veale A, Cohen A, Arazi T(2008) Agrobacterium-mediated transformation of the bulbous flower Omithogalum. Acta Hortic ulturae, vol 766. Seoul

van Rossum MWPC (1997) Role of physiological factors in tulip bulb scale mic ropropagation. Proefsc hrift Wageningen, Van Rossum, [S.I.]

van Tunen AJ, Eikelboom W, Angenent GC (1993) Floral organogenesis in Tulipa. Flower Newsletter 16:33-38

van Tuyl J M, Arens P, Marasek-Ciołakowska A (2012) Breeding and Genetics of Ornamental Geophytes. In: Omamental Geophytes: From Basic Science to Sustainable Production. CRC Press, $p 131$

Van Verk MC, Hickman R, Pieterse CMJ, Van Wees SCM (2013) RNA-Seq: revelation of the messengers. Trends in Plant Science 18 (4):175-179. doi:http://dx.doi.org/10.1016/j.tplants.2013.02.001 
Venturini L, Femarini A, Zenoni S, Tomielli G B, Fa soli M, Santo SD, Minio A, Buson G, Tononi P, Zago ED, Zampenin G, Bellin D, Pezzotti M, Delledonne M (2013) De novo transcriptome cha rac teriza tion of Vitis vinifera cv. Corvina unveils va rietal diversity. BMC Genomic s 14 (1):41

Verdeil J -L, Alemanno L, Niemenak N, Tranbarger T) (2007) Plunipotent versus totipotent plant stem cells: dependence versus autonomy? Trends in Plant Science 12 (6):245-252. doi:http://doi.org/10.1016/j.tplants.2007.04.002

Vieten A, Sa uer M, Brewer PB, Friml J (2007) Molecular and cellular aspects of a uxin-tra nsportmediated development. Trends Plant Sci 12 (4):160-168. doi:10.1016/j.tplants.2007.03.006

Villac orta-Martin C, Núñez de CáceresGonzálezFF, de Ha an J , Huijben K, Passa rinho P, LugassiBen Hamo M, Zaccai M (2015) Whole transcriptome profiling of the vemalization process in Lilium longiflorum (cultivar White Heaven) bulbs. BMC Genomics 16 (1):550. doi:10.1186/s12864-015-1675-1

von Amold S, Sabala I, Bozhkov P, Dyachok J, Filonova L (2002) Developmental pathways of somatic embryogenesis. Plant Cell Tissue Organ Cult 69 (3):233-249. doi:10.1023/a:1015673200621

Vroemen CW, Mord horst AP, Albrecht C, Kwa a itaal MACJ, de Vries SC (2003) The CUP-SHAPED COTYLEDON3 Gene is Required for Boundary and Shoot Meristem Formation in Arabidopsis. The Plant Cell 15 (7):1563-1577. doi:10.1105/tpc.012203

Walker RI (1940) Regeneration in the Scale Leaf of Lilium candidum and L Iongiflonum. American J oumal of Botany 27 (2):114-117. doi:10.2307/2436694

Wan CY, Wilkins TA (1994) A Modified Hot Borate Method Signific antly Enhances the Yield of High-Q ua lity RNA from Cotton (Gossypium hirsutum L.). Ana lytic al Bioc hemistry 223 (1):712. doi:http:// dx.doi.org/10.1006/a bio.1994.1538

Wang G, Fiers M (2010) CLE peptide signaling during plant development. Protoplasma 240 (14):33-43. doi:10.1007/s00709-009-0095-y

Wang J, Tian C, Zhang C, shi b, Cao X, Zhang T-Q, zhao z, Wang J -W, J iao Y (2017) Cytokinin Signaling Activates WUSC HEL Expression during Axillary Meristem Initiation. The Plant Cell. doi:10.1105/tpc .16.00579

Wang J, Yang Y, Liu X, Huang J, Wang Q, Gu J, Lu Y (2014) Transc niptome profiling of the cold response and signaling pathways in Lilium lancifolium. BMC G enomics 15 (1):203

Wang K, Peng H, Lin E, J in Q, Hua X, Yao S, Bian H, Han N, Pan J, Wang J, Deng M, Zhu M (2010) Identific ation of genes related to the development of bamboo rhizome bud.J Exp Bot 61 (2):551-561. doi:10.1093/jxb/erp334

Wang M, Wang G, Ji J , Wang J (2009) The effect of pds gene silencing on chloroplast pigment composition, thylakoid membrane structure and photosynthesis efficiency in tobacco 
$\begin{array}{llll}\text { plants. } & \text { Plant } & \text { Science } & 177\end{array}$

doi:http://dx.doi.org/10.1016/j.plantsc i.2009.04.006

Wang Y, Kronenburg B, Menzel T, Maliepaard C, Shen X, Krens F (2012) Regeneration and Agrobacterium-mediated transformation of multiple lily cultivars (Plant Cell, Tissue and Organ Culture (РCTOC)). 111 (1):113-122. doi:10.1007/s11240-012-0172-3

Wang Z, Cao R, Cheng J (2013) Three-Level Prediction of Protein Function by Combining ProfileSequence Search, Profile-Profile Search, and Doma in Co-Occurrence Networks. BMC Bio informatic 14 (Suppl 3):S3

Ward SP, Leyser O (2004) Shoot branching. Current Opinion in Plant Biology 7 (1):73-78

Watad AA, Yun DJ , Matsumoto T, Niu X, Wu Y, Kononowic zAK, Bressan RA, Hasegawa PM (1998) Mic roprojectile bombardment-mediated transformation of Lilium long iflorum. Plant Cell Rep 17 (4):262-267. doi:10.1007/s002990050389

Wu J, Seng S, Sui J, Vonapartis E, Luo X, Gong B, Lu C, Wu C, Liu C, Zhang F, He J, Yi M (2015) Gladiolus hybridus ABSC ISIC ACID INSENSITIVE 5 (GhABI5) is an important transcription factor in ABA signaling that can enhance Gladiolus com domancy and Arabidopsis seed domancy. Frontiers in Plant Science 6 (960). doi:10.3389/fpls.2015.00960

Xu J , Hofhuis H, Heidstra R, Sauer M, Friml J , Sc heres B (2006) A molec ular framework for plant regeneration. Science 311 (5759):385-388. doi:10.1126/sc ience.1121790

Xu L, Huang H, Brigitte G (2014) Chapter One - Genetic and Epigenetic Controls of Plant Regeneration. In: Current topics in developmental biology, vol Volume 108. Academic Press, pp 1-33. doi:http://dx.doi.org/10.1016/B978-0-12-391498-9.00009-7

Xu Y, Buc hholz W, DeRose R, Hall T(1995) Charac terization of a rice gene fa mily encoding rootspecific proteins. Plant Mol Biol 27 (2):237-248. doi:10.1007/bf00020180

Yadav RK, Girke T, Pasala S, Xie M, Reddy GV (2009) Gene expression map of the Arabidopsis shoot apical meristem stem cell niche. Proc Natl Acad Sci U S A 106 (12):4941-4946. doi:10.1073/pnas.0900843106

Yadav RK, Tavakkoli M, Xie M, Girke T, Reddy GV (2014) A high-resolution gene expression map of the Arabidopsis shoot meristem stem cell niche. Development 141 (13):2735-2744. doi:10.1242/dev.106104

Yan L, Fu D, L C, Blechl A, Tranquilli G, Bonafede M, Sanchez A, Valarik M, Yasuda S, Dubcovsky J (2006) The wheat and barley vemalization gene VRN3 is an orthologue of FT. Proceedings of the National Academy of Sciences 103 (51):19581-19586. doi:10.1073/pnas.0607142103

Yan L, Loukoianov A, Blechl A, Tranquilli G, Ramakrishna W, SanMiguel P, Bennetzen JL, Ec henique V, DubcovskyJ (2004) The Wheat VRN2 Gene Isa Flowering RepressorDownRegulated by Vemalization. Science 303 (5664):1640-1644. doi:10.1126/sc ience.1094305 
Yang F, Wang Q, Sc hmitz G, Müller D, Theres K (2012) The bHUH protein ROX a cts in concert with RAX1 and LAS to modulate axillary meristem formation in Arabidopsis. Plant J oumal 71 (1):61-70. doi:10.1111/j.1365-313X.2012.04970.x

Yang L, Xu M, Koo Y, He J, Poethig RS (2013) Sugar promotes vegetative phase change in Arabidopsis thaliana by repressing the expression of MIR156A and MIR156C. elife 2. doi:10.7554/eLife.00260

Yang Y, Li R, Qi M (2000) In vivo analysis of plant promoters and transcription factors by agroinfiltration of tobacco leaves. The Plant J oumal 22 (6):543-551. doi:10.1046/j.1365313x.2000.00760.x

Yao C, Finlayson SA (2015) Abscisic Acid Isa General Negative Regulator of Arabidopsis Axillary Bud G rowth. Plant Physiology 169 (1):611-626. doi:10.1104/pp.15.00682

Yin Z-F, Zhao B, Bi W-L, Chen L, Wang Q-C (2013) Direct shoot regeneration from basal leaf segments of Lilium and assessment of genetic stability in regenerants by ISSR and AFLP markers. In Vitro Cellular \& Developmental Biology - Plant 49 (3):333-342. doi:10.1007/s11627-013-9501-4

Yu Q, Tian H, Yue K, Liu J, Zhang B, L X, Ding Z(2016) A P-Loop NTPase Regulates Quiescent Center Cell Division and Distal Stem Cell Identity through the Regulation of ROS Homeosta sis in বta lic >A rabidopsis</ ita lic > Root. PLoS Genet 12 (9):e1006175

Yu S, Cao L, Zhou C-M, Zhang T-Q, Lian H, Sun Y, Wu J, Huang J, Wang G, Wang J-W (2013) Sugar is an endogenous cue for juvenile-to-adult phase transition in plants. elife 2. doi:10.7554/eLife.00269

Zhang H, J in J, Tang L, Zhao Y, Gu X, Gao G, Luo J (2011) PlantTFDB 2.0: update and improvement of the comprehensive plant transcription factor database. Nucleic Acids Research 39 (suppl 1):D1114-D1117. doi:10.1093/nar/gkq1141

Zha o Y (2012) Auxin Biosynthesis: A Simple Two-Step Pathway Converts Tryptophan to Indole-3Acetic Acid in Plants. Molecular Plant 5 (2):334-338. doi:https://doi.org/10.1093/mp/ssr104

Zhu Q-H, Helliwell CA (2011) Regulation of flowering time and floral patteming by miR172. J Exp Bot 62 (2):487-495. doi:10.1093/jxb/erq 295 
SUMMARY 
Vegetative propagation is very important for the survival of species with long juvenile and adult vegetative phases, as it is the case for bulbous plants. Bulbous plants are ornamental geophytes with a bulb as an underground storage organ. Among flower bulbs, tulip and lily are the two commercially leading plants in The Netherlands. Tulip propagates vegetatively via axillary bud outgrowth, while lily propagates via adventitious bulblet formation. The vegetative propagation rate in tulip is very low due to the limited amount of axillary buds that will grow successfully. Moreover, tulip is very recalcitrant to in vitro regeneration. On the other hand, lily propagates efficiently via adventitious bulblet formation, either naturally from the underground portion of the stem of the apical bud, or artificially from detached bulb scales.

This thesis study aimed to understand how axillary bud outgrowth is controlled in tulip bulbs and how regeneration capacity is established in lily bulb scales. As a first step towards these goals, the state of the art of the molecular control of sexual and vegetative reproduction was reviewed for model species. Moreover, two approaches, "bottom-up" and "top-down", to transfer the knowledge from model to non-model species were described (Chapter 2). In short, the "bottom-up" approach usually goes from individual genes to systems, assuming conservation of molecular pathways and using sequence homology searches to identify candidate genes. "Top-down" methodologies go from systems to genes, and are based on large scale transcriptome profiling via e.g. microarrays or RNA sequencing, followed by the identification of associations between phenotypes, genes, and gene expression patterns and levels.

Next (Chapter 3), two sets of high quality transcriptomes, one for tulip and one for lily were generated from a collection of several tissues using the Illumina HiSeq 2000 platform. Several assembly filtering parameters were applied, to highlight the limitations of stringent but routinely used filtering in de novo transcriptome assembly. The final created transcriptomes were made publicly available via a user friendly Transcriptome browser (http://www.bioinformatics.nl/bulbs/db/species/index) and their usefulness was exemplified by a search for all potential transcription factors in lily and tulip, with special focus on the TCP transcription factor family.

One TCP member was of special interest because it has proven to integrate several pathways that control axillary bud outgrowth in a wide range of species. It is called TEOSINTE BRANCHED $l$ (TBI) in monocots and BRNACHED 1 (BRC1) in dicots. A Tulipa gesneriana TBI transcript was identified from the generated transcriptome and subsequently, tulip axillary bud outgrowth was studied through a "bottom-up" approach (Chapter 4). The degree of axillary bud outgrowth in tulip determines the success of their vegetative propagation. However the number of axillary meristems in one bulb is low -six on average- and not all of them seem to have the same growth capacity. The combination of physiological and targeted molecular 
experiments indicated that the first two inner located buds do not seem to experience dormancy (assessed by weight increase and TgTB lexpression) at any point of the growth cycle, while mid-located buds enter dormancy by the end of the growing season. Moreover it was shown that TgTBI expression in tulip bulbs can be modulated by sucrose, cytokinin and strigolactone, just as it has been reported for other species. However, the limited growth of midlocated buds even when their TgTBl expression was naturally or artificially downregulated, pointed at other factors, probably physical, inhibiting their growth.

Next, the remarkable regeneration capacity of lily by initiating de novo shoot meristems from excised bulb scales without the addition of exogenous hormones or growth regulators was studied using a "top-down" approach (Chapter 5). An extensive and comprehensive transcriptome set was generated from lily bulb scales in a time-series using two cultivars and two explant types, all differing in regeneration capacity. This set up provided first insight in the key molecular process underlying pro-meristem induction and meristem initiation in lily. We found that wounding activates a very fast regeneration response, probably mediated by APETALA2/ETHYLENE RESPONSIVE FACTORS (AP2/ERF,) sUCh as LOERF115 and WOUND INDUCED DEDIFFERENTIATION 2 (LOWIND2), which in turn might mediate polar auxin re-distribution, cell proliferation and de-differentiation. Moreover, the timing and level of induction of shoot meristem regulators, sUch as ENHANCER OF SHOOT REGENERATION 2 (LOESR2) and SHOOT MERISTEMLESS (LOSTM) correlated with the regeneration capacity of the scale.

Regardless the regeneration capacity of the different explants e.g. cultivar or position within the scale, regeneration occurs at the proximal-adaxial side of the bulb scale, right on top of the excision line. Thus the possible cellular and physiological factors granting lily bulb scales their competence to regenerate was investigated (Chapter 6). We found that the adaxial parenchyma tissue seems to be more competent than the abaxial tissue, partially because of higher number of secondary veins and larger cell population than the abaxial parenchyma region. It was proposed that upon explant excision, the polar auxin transport is disrupted, creating an auxin maximum at the excision line, which might create a gradient of cell divisions favouring the adaxial parenchyma tissue. The direction of this cell division gradient proved to be negatively affected by the absence of the adaxial epidermis. Moreover, explants without epidermis reduced dramatically their regeneration capacity, and lost the typical proximaladaxial orientation of regeneration. Thus, a better understanding of the composition and physiology of the epidermis in lily bulb scales is essential to identify the regeneration stimulating signals originating from this tissue layer in Lilium sp.

Finally in Chapter 7, integration of all the results was done and I addressed how this may contributes to the fundamental and applied understanding of vegetative propagation in bulbous plants. Also, some challenges are discussed, for example, the complexity in the architecture of tulip bulbs and how this influences ways for improving its rate of axillary bud 
Summary

outgrowth. The challenge to prove the findings of this thesis through functional analysis is also discussed and the possibility of using transient virus-induced gene silencing is highlighted. Moreover, the potential of lily bulb scales as a model system to study some aspects of de novo regeneration, as well as to study the recalcitrance of in vitro propagation is highlighted, supporting the idea that more "omics" data and biotechnological tools for bulbous plant research are necessary. 


\section{ACKNOWLEDGEMENTS}


Nobody said it would be easy... neither was I prepared to deal with the frustration that derives from working with orphan species. Nevertheless, this journey would not have been as fun if it wasn't for the warmth, friendship and tons of happy and funny moments I spent with my friends and colleagues at PPH. Many people have contributed to make this 4.5 years a beautiful experience in my life. Cecilia, Deborah, Beatriz, Giovanni, Wei and Esmer, my arrival and integration to PPH was very smooth thank to all of you! I'm very grateful for the nice moments we had all the time, both at work and outside. Cecilia, Deborah and Bea, you quickly became my close friends, we could talk long about many things and those moments are stored forever in my heart. It was hard to live apart from my husband the first two years, but you girls filled my heart with so much love, thanks again! I also want to thank Renake, Kristyna, Elise, Gonda and Mariana for the nice outings and funny chats. And off course, my birthday celebration partner, Esmer, it was very nice to arrange parties together!

I always considered that the diversity of cultures in PPH made people very interactive and caring. I learned a lot from all my colleagues, but I specially admired the always happy looks of Wilco and Julio, the peaceful soul of Lidiya, the optimism of Bea, the strength of Esmer, the kindness of Thierry, the sweetness of Elise, the efficiency of Leo and Melissa, the accuracy of Bas and Juriaan; and the politeness of Richard, Juriaan, Arman and Nafiseh. Also, thank you Leonie, Henk, Anderson and Marco (biosciences) for asking me often if I was doing and feeling fine when I was pregnant. I highly valued those little details! Also, to my mates in the silent room,

Gonda, Elise, Yuanyuan and Mahadere, thanks for all the silent and no so silent moments shared. Off course, I also need to thank Yunmeng, Emilie, Mahadere, Leonie and Leo for sharing their pregnancy (of your wife, Leo) and parenting experiences with me.

Also, I will never forget the $\mathrm{PhD}$ trip and our incident at the Swiss border.... I have never been so scared in my life when of a Korean driver (Sangsok), driving a van with a Chinese man without passport (June), a French (Elise), Spanish (Bea), Colombian (me), and Iranian (Farzaneh) women, dared to push the gas when asked to pull over by the border control officer. But hey Sangsok, at least I have now a funny story to tell my son! Thanks for that, hahaha. Thank you guys of PPH also for attending my alcohol tasting workshops at the multi-cultural parties, even though I don't know much about alcohol. Esmer, thanks for asking me (and trusting on me) to give a dancing workshop at your wedding, even when you know that I like to improvise in my workshops! Also, Rina and Harro, thanks for inviting me to join the PPH activity committee. 
Many thanks to Bas, Thierry, Julio and Anderson who were always willing to make some time to discuss scientific ideas related to my thesis. Also to the people that helped me quite often with technical lab and software issues, such as Wilco, Bas, Leo, Juriaan, Lidyia, Bea, Melissa, Renake and Kristyna. I learned a lot from all of you! My special thanks to the bioinformaticians Edouard and Harm, because you had the patience of a saint to teach and explain me the principles of some complicated analysis. Also Harm, thanks for giving me personal advices, you do not only know about bioinformatics, you know a lot about life.

Richard, thanks for your supervision and your support. Although I did not have a strong background in molecular biology, you believed in me and managed to convince me that fundamental research is very important even when no short term applications result from it. I still believe that fundamental and applied research go hand to hand and that one does not necessarily exclude the other. But thanks to you, now I know that solid fundamental knowledge is the pillar of applied research. Also, I admire your politeness and down to earth attitude. I am sure that many more achievements will come soon in your career! Henk, thanks for letting us being part of your WSL group and for facilitating a proper work environment. Also, thanks for caring about the personal lives of your personnel, I loved the gift you and Leonie gave to Emilio for his first birthday!

Special thanks also go to my students, which played a fundamental role for my personal and academic development. Aimee, Marta, Chantal, Aneemarieke, Anne and Eveline, thank you for all your effort, and willingness to learn. I also learned tons from you! Also, thanks Maarten Holdinga and Alex van Silfhout for the help with managing the bulbs. The external committee, Henk Gude, Bas Brandwagt, Carlo Randag, Sjaak Langeslag, Paul Bijman and Danielle Kroes from the KAVB, who every six months evaluated this project progress, thanks for the encouragement to continue our research!. Paul Arens and Geert-Jan de Klerk thank you also for all the input and informal discussions we had, you two helped me a lot to put me into context in the bulb field. Wouter and Ikram, I cannot thank you enough for all your help! thanks for teaching me SEM (Wouter), confocal microscopy and in situ hybridization (Ikram).

To my outside-work friends, which I call them my extended family, what a great gift I had to be back in Wageningen and spend more time with all of you! Most of us met during our MSc studies, got married, got jobs, got kids and most important, got bounds forever. Caro and Roland, Paula and Cesar, Marce and Rafa, Ale, Daisy, Lore, Isa and Carlos, Caro and Robert, Indi and Chris, Andrea and Matthijs, Claudia and Eddie, Sergio and Madeleine, Carlos and Carla, Pieter and Vicky, Claudia and Christiaan, thanks for all the trips, birthday celebrations, dinners and gatherings. I have the feeling that no matter where we end up, I will always have a special bond to all of you. My special acknowledgments to Paula, Cesar, Ale, Isa, "don 
Carlitos", Marce and Rafa for giving me a hand with Emilio while I was finishing my thesis and when I was fixing my post-partum anxiety. Being far from my family at those critical times was more bearable because of your help.

Regarding my family, I could not have finished my thesis on time without the great help of my mom, my parents in law and my husband. Mami, gracias por tu incondicional ayuda durante mi licencia de maternidad y durante los tres meses adicionales que estuviste con nosotros en 2017. Llegar a casa y encontrar comida caliente y deliciosa, la casa limpia y a Emilio bien cuidado, fue una gran bendición y un factor determinante para mi recuperación y finalizacion de mi tesis. Gracias por todo lo que haces por mi!. Papi, gracias por ayudarnos y acompañarnos durante los primeros meses de vida de Emilio y por todos tus consejos sobre crecimiento personal. Mijn schoon ouders Willy en Linda, Ik apprecier het heel erg dat jullie elke week vit België komt om Emilio te verzorgen. Ook, hartelijk bedankt voor jullie gezelschap in de momenten dat Bjorn op lange reis is. Het voelt voor mij ook een beetje als familie zo, ook al in mijn familie heel erg weg.

Almost last but not less important, thank you my dear best friend, life companion, soul mate and husband, Bjorn. Thanks for understanding how important this choice of doing a PhD was for me; for making me believe that this dream was possible; for cheering me up when I was frustrated; for making my life easier during the hard days at work; for enjoying together the simple but fulfilling things in life such as admiring a sunset, learning to cook a nice meal or watching a funny tv show. Thanks for having sacrificed so many hours in traffic when commuting to work, so we could live together. Finally, thank you for having always believed in me, and transmitting me the certainty that I will always achieve my goals and dreams.

Last, Emilio, the new engine of my heart, thanks for choosing me as your mother. I finally understood what really is important in life because of you. This book is dedicated to you, because you gave me the inspiration to finish my $\mathrm{PhD}$ on schedule. It was amazing how you completely renewed my energy after the long and hard days at work. Finally, thanks for teaching me that unconditional love is all we need to flourish.

For sure I am forgetting to mention more people in this section, sorry for that, but all of you that made me smile in this 4.5 years are for sure already acknowledged by my heart. 
CURRICULUM VITAE 


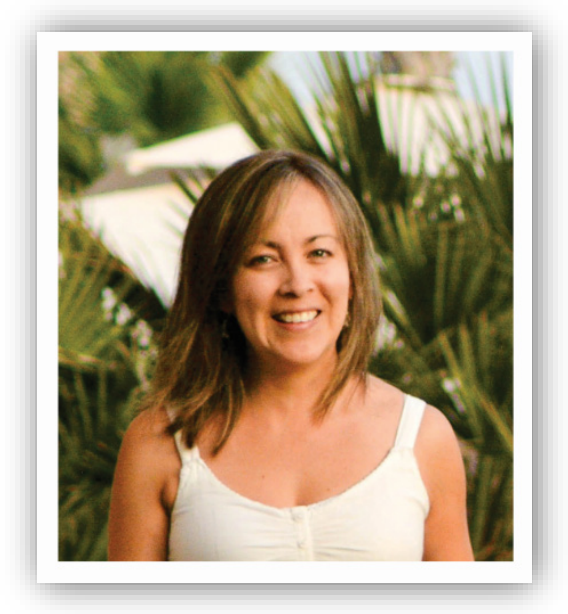

Natalia was born in Medellín, Colombia the $23^{\text {rd }}$ of September of 1981. During her childhood she developed a special interest for nature and therefore Biology became her favourite class at school. To further develop her scientific interest, she chose a scientific emphasis at high school and successfully graduated from "Gimnasio los Pinares" in 1998. In 1999 she enrolled in an English program in South Bend, Indiana, USA, where she had close contact with the University of Notre Dame. This experience made Natalia aware of the rich cultural and scientific background of the academic environment. When returning home, she enrolled into medical school and became an active member of the cell biology student club. The scientific discussions at this club made Natalia very interested in the enormous potential of nature to improve the human health. In 2001 Natalia decided to switch major and moved to Bogotá, the capital of Colombia, to study Biology with emphasis in plant biotechnology. At the end of her studies, she decided to do a one year BSc thesis at the International Centre for Tropical Agriculture (CIAT) in Palmira. During her thesis she characterized the nutritional content and genetic diversity of common bean (Phaseolus vulgaris) seeds. After her BSC graduation, she was hired in the same laboratory as a research assistant, to follow up the increase of iron and zinc content in common bean seeds, with the aim to reduce the levels of nutritional deficiency of people living in poor areas. As part of her job, she travelled to the Institute for Genomic Diversity (IGD) of Cornell University, USA in 2008, where she got trained for six months into molecular and breeding analysis. In 2009 Natalia got granted a fellowship to pursue a master in Plant Science with emphasis in Plant Breeding at Wageningen University in the Netherlands. This master provided Natalia more bases of genetics, genomics, statistics and bioinformatics knowledge. In 2011 Natalia took a traineeship at the vegetable breeding company Rijk Zwaan, and in 2012 worked at Armada, a small flower breeding company. Following her passion for research, at the end of 2012 she enrolled in a PhD position at the endowed chair of Physiology of Flower Bulbs at the department of Plant Physiology of Wageningen University \& Research, under the supervision of prof. dr. Richard Immink. During the next 4.5 years Natalia worked deciphering the molecular regulation of vegetative propagation in tulip and lily bulbs. Her work can be found in this book. This academic experience has made Natalia grow as an independent researcher but it also made her grow in her personal development. 
LIST OF PUBLICATIONS 
Leeggangers, H.A.C.F.*; Moreno-Pachon, N.M.*; Gude, H.; Immink R.G.H. 2013. Transfer of knowledge about flowering and vegetative propagation from model species to bulbous plants. The International journal of developmental biology 57(6-8):611-20. Doi: 10.1387/ijdb.130238ri.

Basnet, R.K.; Moreno-Pachón, N.M.; Lin, K.; Bucher, J.; Visser, R.G.F.; Maliepaard, C.A.; Bonnema, A.B. 2013. Genome-wide analysis of coordinated transcript abundance during seed development in different Brassica rapa morphotypes. BMC Genomics 14:840.

Moreno-Pachon, N.M.*; Leeggangers, H.A.C.F*; Nijveen, H*.; Severing, E.; Hilhorst, H.W.M.; Immink, R.G.H. 2016. Elucidating and mining the Tulipa and Lilium transcriptomes. Plant Molecular Biology 92(3):249-61. Doi: 10.1007/s11103-016-0508-1.

Moreno-Pachon, N.M.; Mutimawurugo, M.C.; Heynen, E.; Sergeeva, L.; Blilou, I.; Hilhorst, H.W.M.; Immink, R.G.H. 2016. Role of Tulipa gesneriana TEOSINTE BRANCHED 1 (TgTBI) in the control of axillary bud outgrowth in bulbs. Submitted to the Plant Reproduction journal.

* Authors contributed equally to this work 
EDUCATION STATEMENT 


\section{Education Statement of the Graduate School \\ Experimental Plant Sciences}

Issued to: Natalia Maria Moreno Pachon

Date: $\quad 12$ October 2017

Group: Plant Physiology

University: Wageningen University \& Research

\section{1) Start-up phase}

- First presentation of your project

Title: Project proposal Molecular regulation of flower bulb propagation

Writing or rewriting a project proposal

Molecular regulation of flower bulb propagation

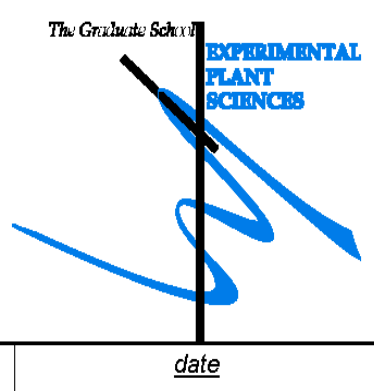

- Writing a review or book chapter

Titte: Transfer of knowedge about flowering and propagation from model species to bulbous plants, Int.

J. Dev. Biol. 57 pp611-620 (2013). DOl:10.1387/fidb.130238ri

22 Apr 2013

MSc courses

Laboratory use of isotopes

Subtotal Start-up Phase

13.5 credits $^{\star}$

2) Scientific Exposure

- EPS PhD student days

EPS PhD student day, Leiden, NL

EPS PhD 'Get2gether', Soest, NL

- EPS theme symposia

EPS theme 1 symposium "Developmental Biology of Plants" Leiden, NL

EPS theme 3 symposium "Metabolism and Adaptation" Amsterdam, NL

EPS theme 1 symposium "Developmental Biology of Plants" Wageningen, NL

EPS theme 1 symposium "Developmental Biology of Plants" Leiden, NL

EPS theme 1 symposium "Developmental Biology of Plants" Wageningen, NL

EPS theme 1 symposium "Developmental Biology of Plants" Leiden, NL

- National meetings (e.g. Lunteren days) and other National Platforms

Annual meeting "Experimental Plant Sciences", Lunteren, NL

Annual meeting "Experimental Plant Sciences", Lunteren, NL

Annual meeting "Experimental Plant Sciences", Lunteren, NL

Annual meeting "Experimental Plant Sciences", Lunteren, NL

date

- Seminars (series), workshops and symposia

Flying seminar series

"Tackling natural variance in seed metabolism integrating metabolite profiles via network analysis" (Aaron Fait)

"Arabidopsis thaliana as a model system for the study of evolutionary questions" (Detflet Weigel)

"Genetics and Epigenetics: a Complex Relationship" (Ortrun Mittelsten Scheid)

"The Evolutionary Significance of Gene and Genome Duplications" (Yves van de Peer)

"Regulation of Root Morphogenesis in Tomato Species in the face of a Changing Environment" (Siobhan

Brady)

Seminar series Plant sciences

"Synthetic Biology of Plant Metabolites" (Jules Beekwilder)

"Using the non-model genus Passiflora to study the evolution of novelty in plant reproductive

Symposia

Symposium "Omics Advances for Academia and Industry - Towards True Molecular Plant Breeding"

- Seminar plus

prof Dr Jil Farrant "resurrection plants"

Feb 2012

Mar 2013

29 Nov 2013

28-29 Jan 2015

17 Jan 2013

22 Mar 2013

$24 \operatorname{Jan} 2014$

08 Jan 2015

21 Jan 2016

28 Feb 2017

22-23 Apr 2013

14-15 Apr 2014

13-14 Apr 2015

10-11 Apr 2017

04 Dec 2012

$27 \mathrm{Feb} 2013$

19 Nov 2014

03 Feb 2015

09 Sep 2015

11 Jun 2013

27 Jan 2015

11 Dec 2014

Jul 2016

- International symposia and congresses

Auxins and Cytokinins in Plant Development

Strigolactones

- Presentations

Poster: "Molecular mechanisms underlying Lily bulblet regeneration" at ALW meeting

Poster: "Elucidating the control of axillary bud outgrowth in tulip bulbs" at ALW meeting

Talk: "do novo shoot meristem regeneration from lily bulb scales" at PhD trip

Talk: "Elucidating the mechanism of bulblet regeneration in lily bulb- scales" at Nederlandse Vereniging voor Plantenbiotechnologie en -Weefselkweek

Talk: "Elucidating the mechanism of de novo meristem regeneration in lily bulb scales" at PhD school

Talk: "Elucidating the molecular control of meristem regeneration in lily bulbs" at EPS theme 1

- IAB interview

- Excursions

PPH PhD trip to Germany and Switzerland

Subtotal Scientific Exposure

29 Jun-04 Jul 2014

01-06 Mar 2015

22-23 Apr 2013

13-14 Apr 2015

22 Apr-01 May 2015

05 Jun 2015

05-07 Oct 2016

28 Feb 2017 


\section{3) In-Depth Studies}

date

\section{EPS courses or other PhD courses}

Workshop Genome Mining, Wageningen, NL

Bioinformatics, a user's approach, Wageningen, NL

The power of RNAseq, Wageningen, NL

Transcription Factors and Transcription Regulation, Wageningen, NL

Introduction to $\mathrm{R}$, Wageningen, $\mathrm{NL}$

PhD school Plant Development, Retzbach, Germany

Journal club

PPH literature discusion

Individual research training

Ben Schere's lab Wageningen

\begin{tabular}{|c|c|}
\hline date \\
& 13 Dec 2012 \\
$04-08 \mathrm{Mar} 2013$ \\
$05-07$ Jun 2013 \\
$17-19$ Dec 2013 \\
$23-24$ Oct 2014 \\
$05-07$ Oct 2016 \\
& $2012-2017$ \\
& $24-28$ Oct 2013 \\
\hline Subtotal In-Depth Studies & 9.4 credits $^{\star}$ \\
\hline
\end{tabular}

4) Personal development

- Skill training courses

Working with EndNote X5 (ILP), Wageningen, NL

ExPectationS day "creative thinking", Wageningen, NL

Project and time management (PTM), Wageningen, NL

Reviewing a scientific paper (RSP), Wageningen, NL

Scientific Writing (SW 1-7), Wageningen, NL

Competence Assesment (CAO), Wageningen, NL

Career assessment (CA), Wageningen, NL

Organisation of $\mathrm{PhD}$ students day, course or conference

- Membership of Board, Committee or PhD council

\section{$\underline{\text { date }}$}

06 Dec 2012

$01 \mathrm{Feb} 2013$

Sep-Oct, 2013

06 Nov 2014

Sep-Nov, 2014

Jan-Feb, 2013

12 Jul 2016

Herewith the Graduate School declares that the $\mathrm{PhD}$ candidate has complied with the educational requirements set by the Educational Committee of EPS which comprises of a minimum total of 30 ECTS credits

${ }^{*} A$ credit represents a normative study load of 28 hours of study. 


\section{COLOPHON}

This work was performed at the Laboratory of Plant Physiology, Wageningen University. This research was supported by the Koninklijke Algemeene Vereeniging voor Bloembollencultuur (KAVB), TTI-Green Genetics and the Dutch Ministry of Economic Affairs.

Cover concept design: Natalia M. Moreno-Pachón

Cover layout and design: Paola Andrea Camargo Bello | www.paolacamargo.com

SEM image of lily meristem: taken by Wouter Kohlen

Printed by ProefschriftMaken | Proefschriftmaken.nl 